\title{
Three-component Synthesis of Isoquinoline Derivatives by a Relay Catalysis with a Single Rhodium(III) Catalyst
}

Chao Zhou, Jijun Jiang, and Jun Wang*

Key Laboratory of Bioinorganic and Synthetic Chemistry of Ministry of Education, School of Chemistry, Sun Yat-Sen University, Guangzhou, 510275, P. R. China

\section{Table of Contents}

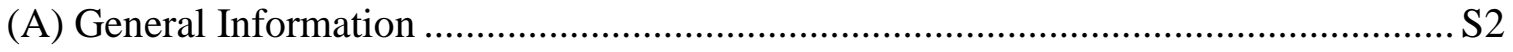

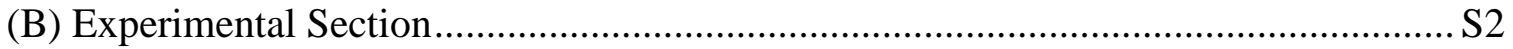

(1) General procedure to prepare $\mathrm{N}$-methoxybenzamide ..................................... S2

(2) General procedure for the preparation of $\alpha$-diazoesters ...................................S3

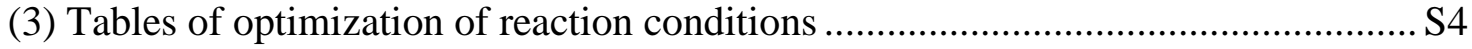

(4) Typical procedure for the catalytic three-component reaction ...........................S7

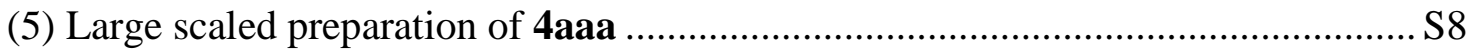

(6) Transformation of the product 4aam to confirm its structure ..............................S8

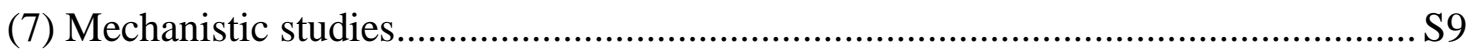

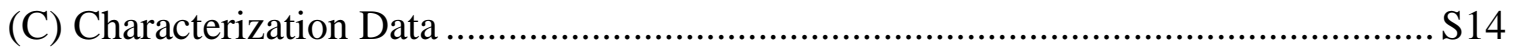

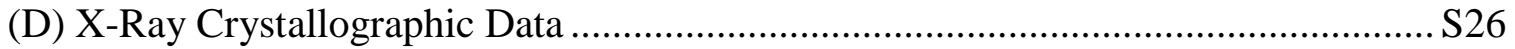

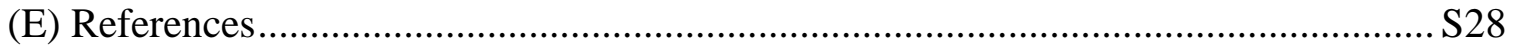

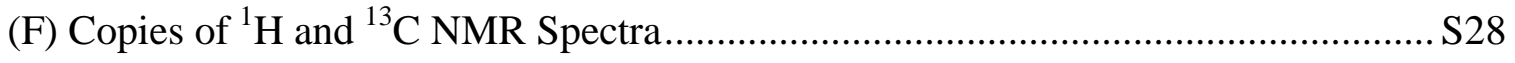




\section{(A) General Information}

Unless specified otherwise, all reactions were carried out under nitrogen atmosphere using dried glassware. $\mathrm{MeOH}$, EtOH, DCM, $\mathrm{Et}_{2} \mathrm{O}$, 1,4-dioxane, toluene, THF and acetone were dried and distilled according to the standard procedures before use. Diphenylacetylenes ${ }^{[1]}$ were synthesized according to literature procedures. Analytical thin layer chromatography (TLC) was performed on precoated silica gel $60 \mathrm{~F}_{254}$ plates. Compounds were visualized by exposure to UV light. Flash column chromatography was performed with silica gel (300-400 mesh). NMR spectra were recorded on $400 \mathrm{MHz}$ spectrometer at ambient temperature, and chemical shifts were given in dimensionless $\delta$ values and were frequency referenced relative to TMS in ${ }^{1} \mathrm{H}$ and ${ }^{13} \mathrm{C}$ NMR spectroscopy. The peak patterns are indicated as follows: s, singlet; d, doublet; $\mathbf{t}$, triplet; $\mathbf{m}$, multiplet; $\mathbf{q}$, quartet. The coupling constants, $J$, are reported in Hertz (Hz). HRMS data were recorded on an ESI -TOF mass spectrometer.

\section{(B) Experimental Section}

\section{(1) General procedure to prepare $N$-methoxybenzamide ${ }^{[2]}$}

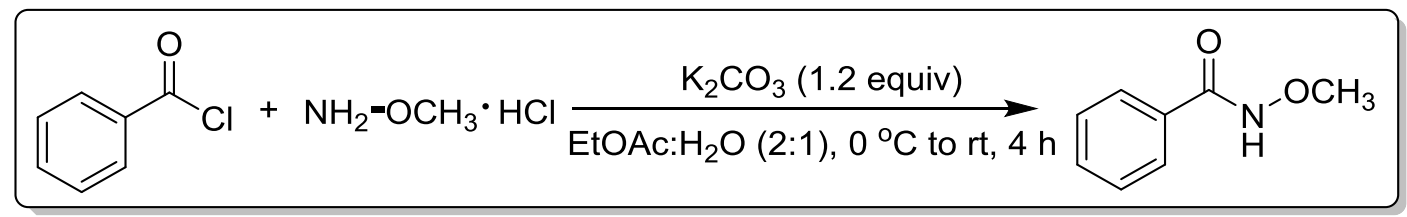

To a solution of $\mathrm{K}_{2} \mathrm{CO}_{3}(1.66 \mathrm{~g}, 12.0 \mathrm{mmol})$ in a mixture of EtOAc/ $\mathrm{H}_{2} \mathrm{O}(2: 1,30 \mathrm{~mL})$ was added methoxyammonium chloride $(1.00 \mathrm{~g}, 12.0 \mathrm{mmol})$. The resulting solution was cooled to $0{ }^{\circ} \mathrm{C}$, followed by dropwise addition of the benzoyl chloride $(1.2 \mathrm{~mL}, 10 \mathrm{mmol})$. The reaction mixture was warmed to room temperature and stirred for $4 \mathrm{~h}$. The organic phase was separated and the aqueous phase was extracted with EtOAc $(15 \mathrm{~mL} \times 3)$. The combined organic layers were dried over $\mathrm{Na}_{2} \mathrm{SO}_{4}$, filtered and evaporated under reduced pressure. The residue was purified by silica gel column chromatography (petroleum ether: ethyl acetate $=3: 1$ ) to give the $N$-methoxybenzamide in $97 \%$ yield as a white solid. 


\section{(2) General procedure for the preparation of $\alpha$-diazoesters}

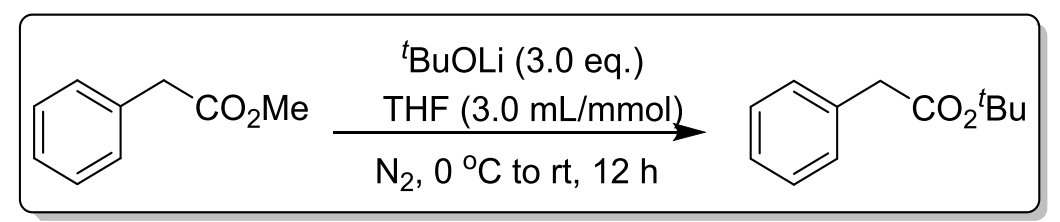

General Procedure for the preparation of tert-Butyl 2-phenylacetate: ${ }^{[3]}$ A solution of lithium tert-butoxide $(2.40 \mathrm{~g}, 30.0 \mathrm{mmol})$ in anhydrous THF $(30.0 \mathrm{~mL})$ was cooled to $0{ }^{\circ} \mathrm{C}$ under nitrogen atmosphere. A solution of methyl phenylacetate $(1.50 \mathrm{~g}, 10.0 \mathrm{mmol})$ in anhydrous THF $(5.0 \mathrm{~mL})$ was added and the reaction mixture was warmed to room temperature and stirred for $12 \mathrm{~h}$. Then, the solvent was evaporated and the organic phase was separated. The aqueous phase was extracted with EtOAc $(10 \mathrm{~mL} \times 3)$. The combined organic layers were dried over $\mathrm{Na}_{2} \mathrm{SO}_{4}$, filtered and concentrated. The residue was purified by silica gel column chromatography (petroleum ether: ethyl acetate $=100: 1$ ) to give the product as a colorless liquid (76\% yield).

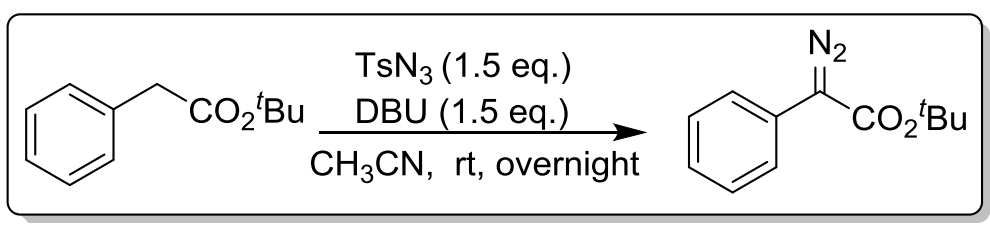

General Procedure for the preparation of tert-Butyl 2-diazo-2-phenylacetate: ${ }^{[3]}$ tert-Butyl 2-phenylacetate $(0.96 \mathrm{~g}, 5.0 \mathrm{mmol})$ and tosyl azide $(1.48 \mathrm{~g}, 7.5 \mathrm{mmol})$ were dissolved in anhydrous acetonitrile $(15.0 \mathrm{~mL})$. DBU $(1.1 \mathrm{~mL}, 7.5 \mathrm{mmol})$ was added dropwise and the resulting mixture was stirred at room temperature for $12 \mathrm{~h}$. The reaction mixture was diluted with distilled water $(10.0 \mathrm{~mL})$, followed by extraction with diethyl ether $(3 \times 15 \mathrm{~mL})$. The combined organic layers were dried over $\mathrm{MgSO}_{4}$, filtered and concentrated. The residue was purified by silica gel column chromatography (petroleum ether: ethyl acetate $=100: 1)$ to give the product as a red-orange oil ( $81 \%$ yield). Note: $\alpha$-diazoesters are better to be used as freshly as possible to ensure satisfied results. 


\section{(3) Tables of optimization of reaction conditions}

Table S1. Screening solvents ${ }^{[\mathrm{a}]}$

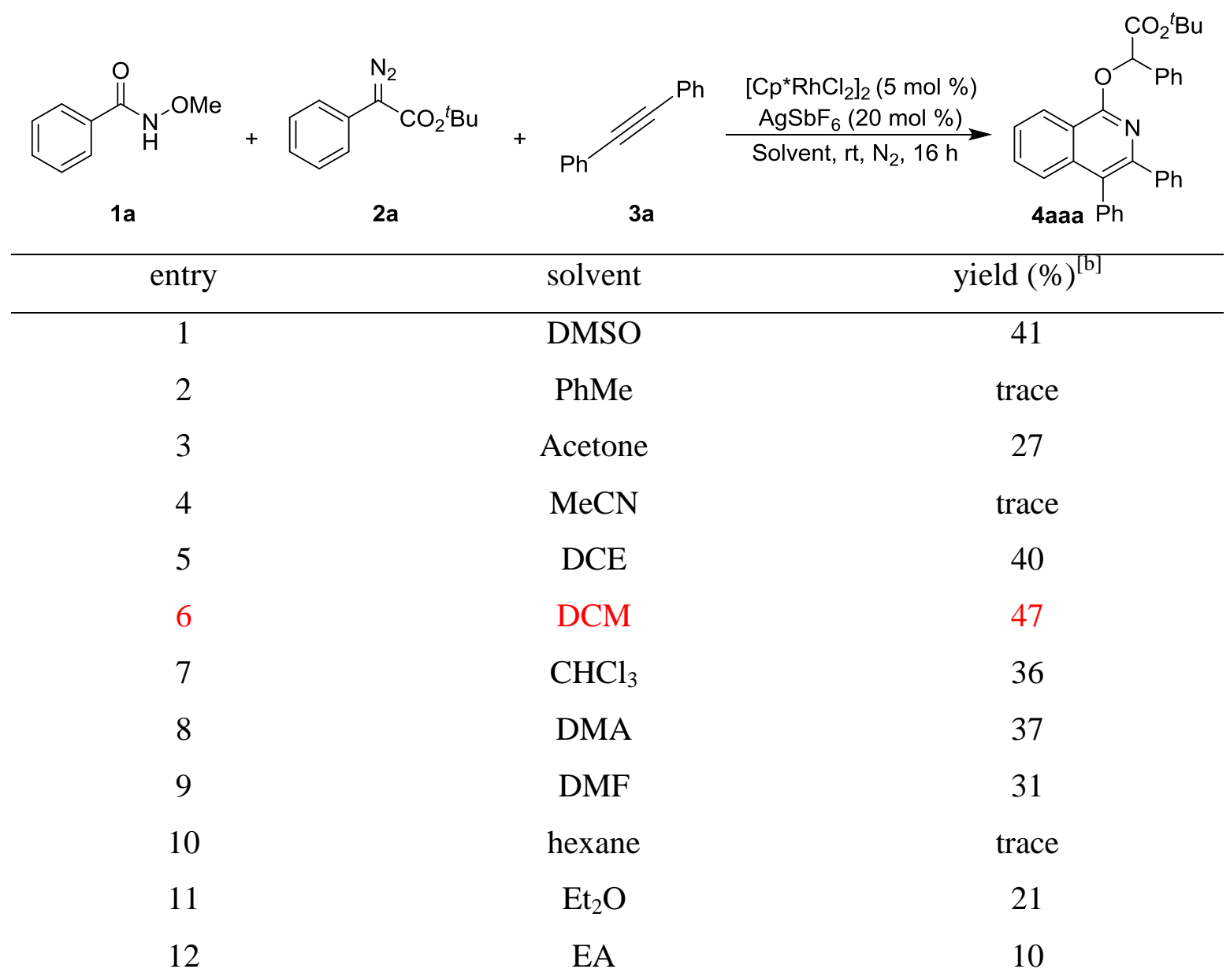

\footnotetext{
${ }^{[a]}$ All reactions were carried out with $19(0.10 \mathrm{mmol}), \mathbf{2 a}(1.0$ eq. $), 3 a(2.0$ eq. $),\left[\mathrm{Cp} * \mathrm{RhCl}_{2}\right]_{2}(5$ mol \%), $\operatorname{AgSbF}_{6}(20 \mathrm{~mol} \%)$ in solvent $(0.2 \mathrm{~mL})$ at room temperature under nitrogen atmosphere for 16 h. ${ }^{[b]}$ Isolated yield.
}

Table S2. Screening the ratio of reactants ${ }^{[\mathrm{a}]}$

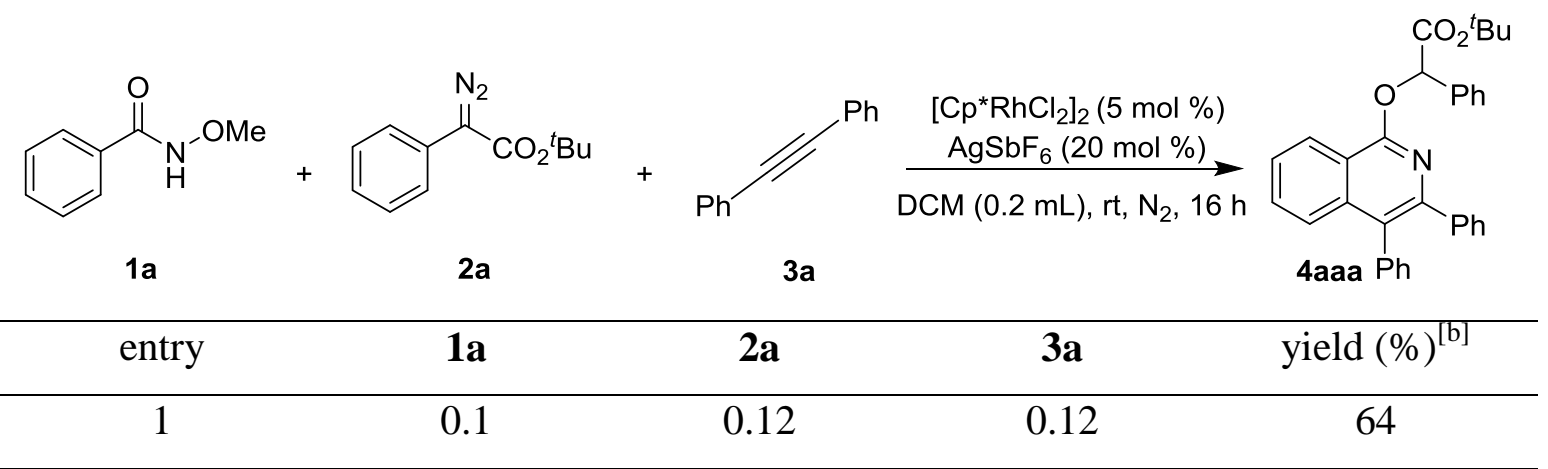




\begin{tabular}{ccccc}
\hline 2 & 0.1 & 0.15 & 0.12 & 67 \\
3 & 0.1 & 0.12 & 0.15 & 70 \\
\hline
\end{tabular}
${ }^{[a]}$ All reactions were carried out with 1a $(0.10 \mathrm{mmol}), \mathbf{2 a}, \mathbf{3 a},\left[\mathrm{Cp}^{*} \mathrm{RhCl}_{2}\right]_{2}(5 \mathrm{~mol} \%), \mathrm{AgSbF}_{6}(20$
mol \%) in DCM $(0.2 \mathrm{~mL})$ at room temperature under nitrogen atmosphere for $16 \mathrm{~h} .{ }^{\left[{ }^{b}\right]}$ Isolated yield.

Table S3. Screening silver salts ${ }^{[\mathrm{a}]}$

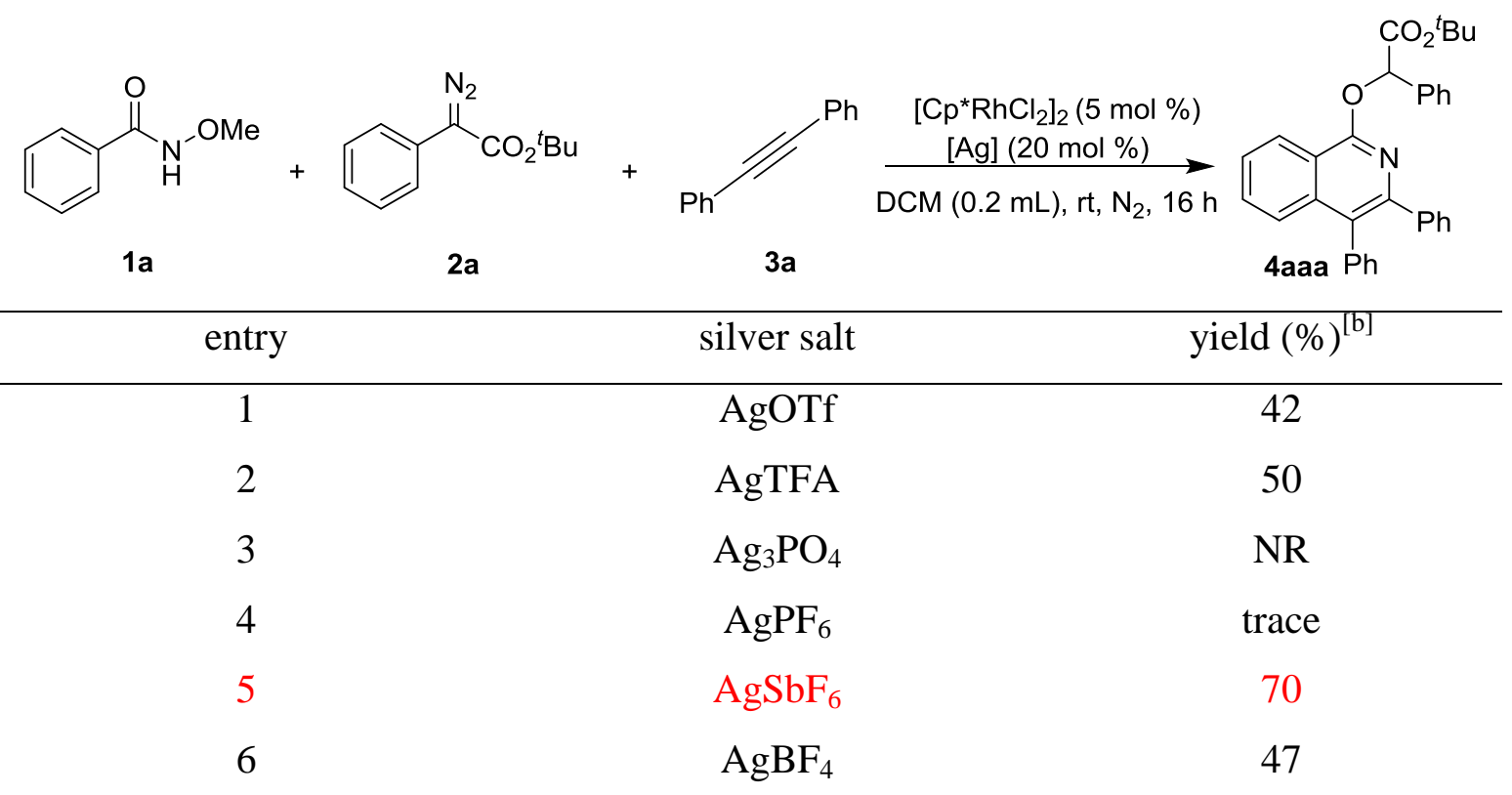

${ }^{[a]}$ All reactions were carried out with 1a $(0.10 \mathrm{mmol}), \mathbf{2 a}(1.2$ eq. $), 3 a(1.5$ eq. $),\left[\mathrm{Cp} * \mathrm{RhCl}_{2}\right]_{2}(5$ mol \%), $[\mathrm{Ag}](20 \mathrm{~mol} \%)$ in DCM $(0.2 \mathrm{~mL})$ at room temperature under nitrogen atmosphere for $16 \mathrm{~h}$.

${ }^{[\mathrm{b}]}$ Isolated yield.

Table S4. Screening reaction time ${ }^{[\mathrm{a}]}$

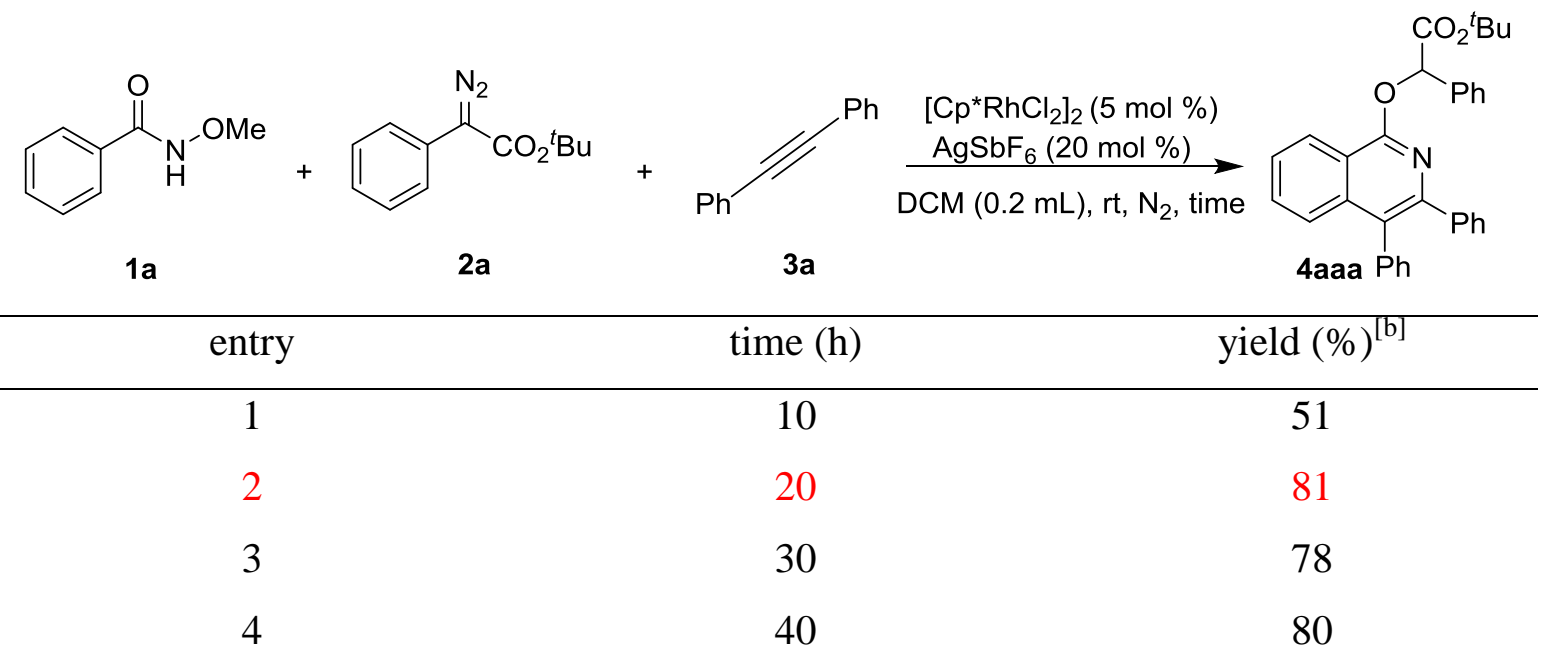

${ }^{\lceil\mathrm{a}]}$ All reactions were carried out with $\mathbf{1 a}(0.10 \mathrm{mmol}), \mathbf{2 a}(1.2$ eq. $), 3 \mathbf{3 a}(1.5$ eq. $),\left[\mathrm{Cp}^{*} \mathrm{RhCl}_{2}\right]_{2}(5$ mol \%), $\operatorname{AgSbF}_{6}(20 \mathrm{~mol} \%)$ in $\mathrm{DCM}(0.2 \mathrm{~mL})$ at room temperature under nitrogen atmosphere. 
${ }^{[b]}$ Isolated yield.

Table S5. Screening reaction temperature ${ }^{[\mathrm{a}]}$

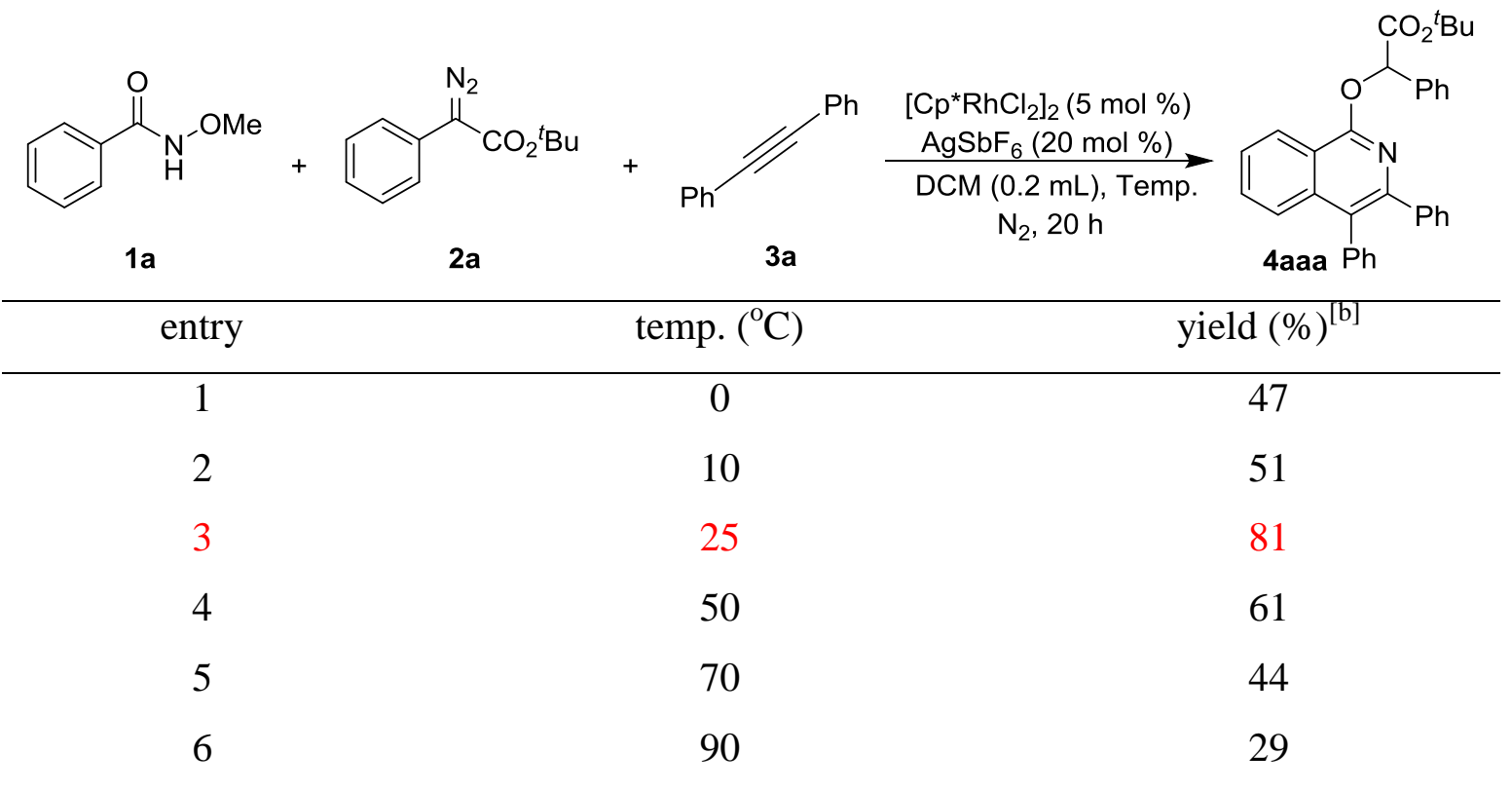

${ }^{\lceil a}$ All reactions were carried out with $\mathbf{1 a}(0.10 \mathrm{mmol}), \mathbf{2 a}\left(1.2\right.$ eq.), 3a $\left(1.5\right.$ eq.), $\left[\mathrm{Cp}^{*} \mathrm{RhCl}_{2}\right]_{2}(5$ mol \%), $\mathrm{AgSbF}_{6}(20 \mathrm{~mol} \%)$ in DCM $(0.2 \mathrm{~mL})$ under nitrogen atmosphere for $20 \mathrm{~h} .{ }^{[\mathrm{b}]}$ Isolated yield.

Table S6. Screening the amount of $[\mathrm{Rh}]^{[\mathrm{a}]}$

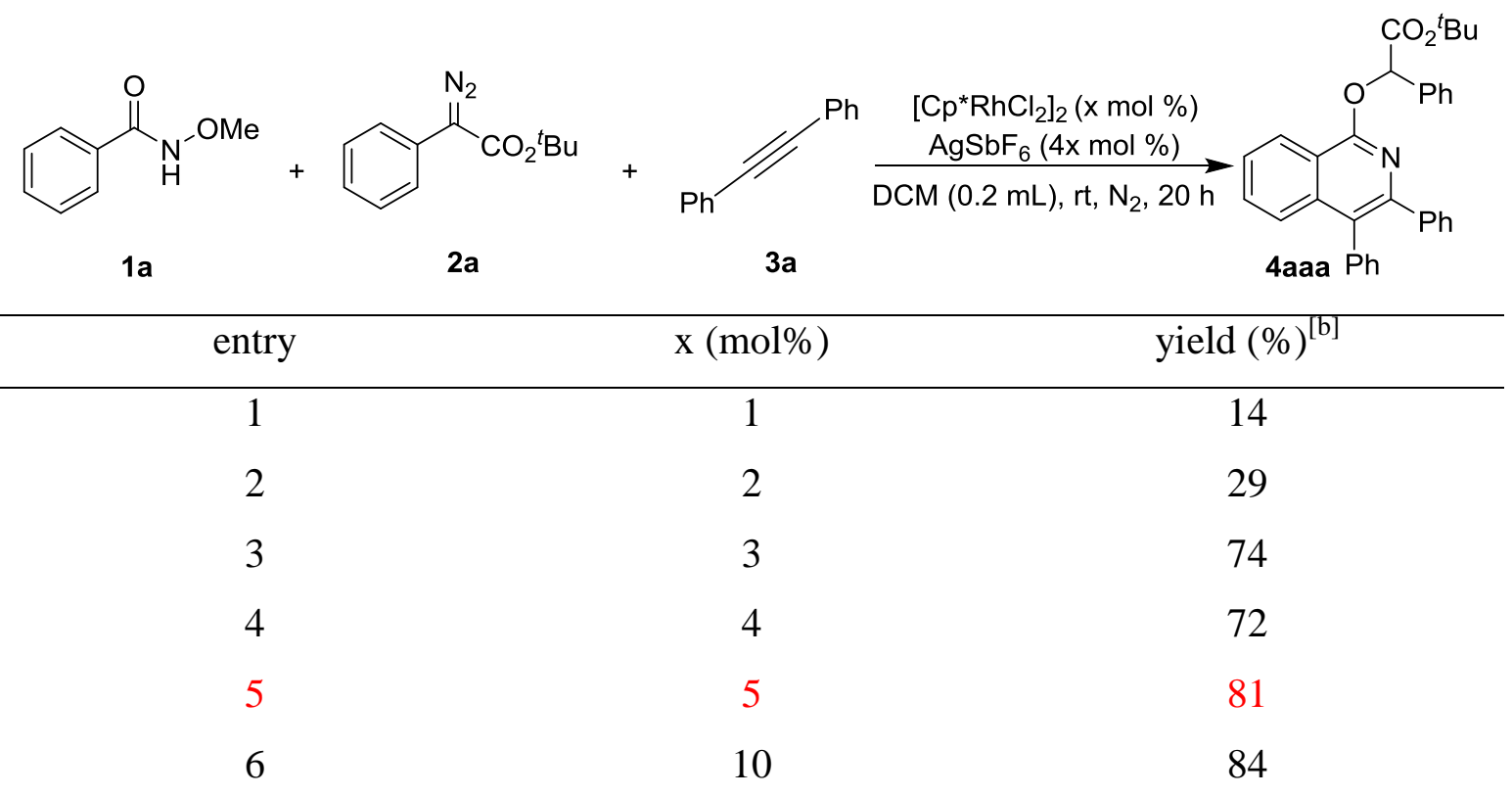

\footnotetext{
${ }^{\text {aa }}$ All reactions were carried out with 1a $(0.10 \mathrm{mmol}), 2 \mathrm{2a}(1.2$ eq. $), 3 \mathrm{a}(1.5$ eq. $),\left[\mathrm{Cp}^{*} \mathrm{RhCl}_{2}\right]_{2}(\mathrm{x}$ mol \%), $\operatorname{AgSbF}_{6}(4 \mathrm{x} \mathrm{mol} \%)$ in $\mathrm{DCM}(0.2 \mathrm{~mL})$ at room temperature under nitrogen atmosphere for 20 h. ${ }^{[\mathrm{b}]}$ Isolated yield.
} 
Table S7. Screening the amount of solvent ${ }^{[a]}$

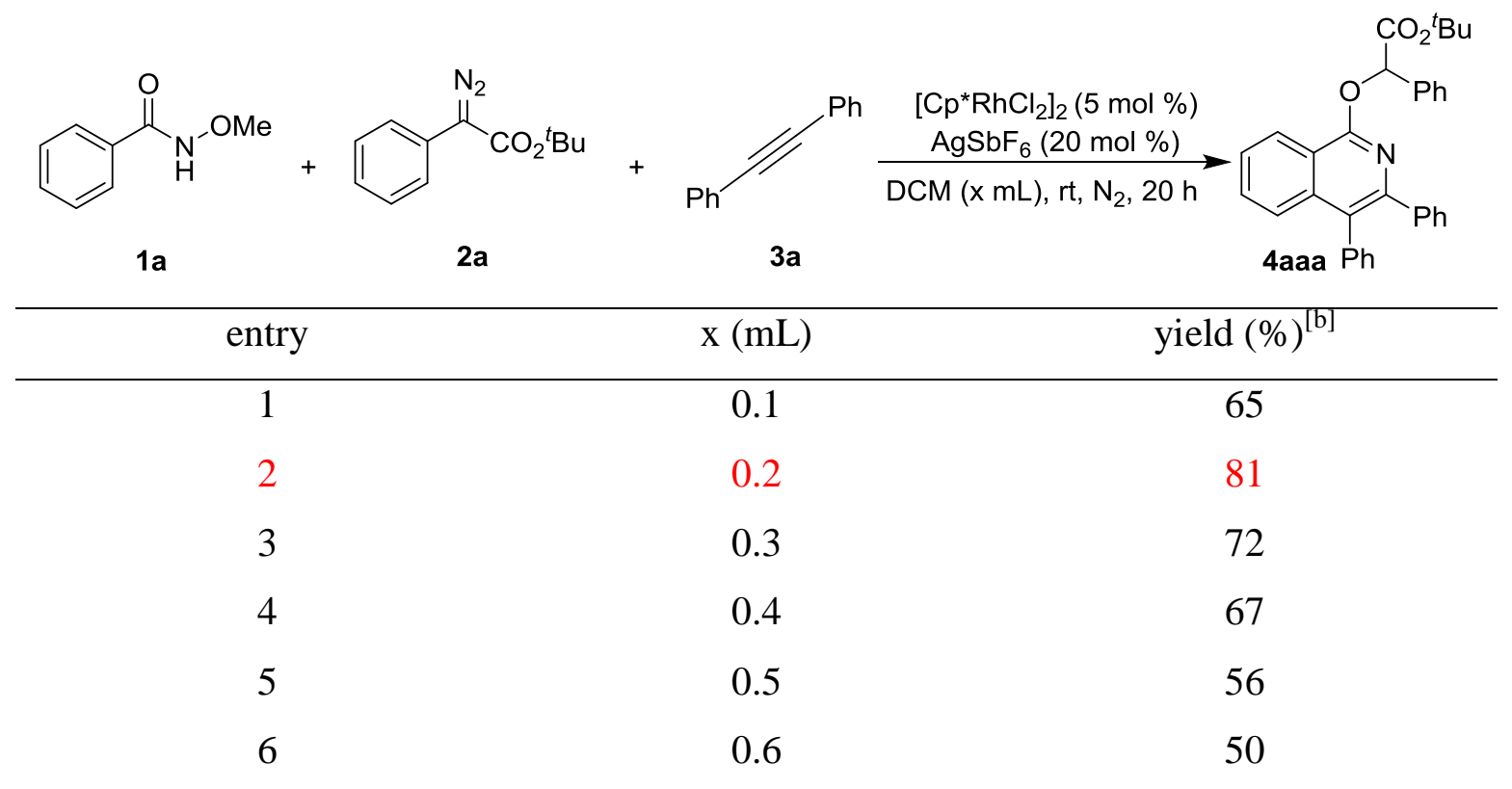

${ }^{[a]}$ All reactions were carried out with $1 \mathrm{a}(0.10 \mathrm{mmol}), \mathbf{2 a}(1.2$ eq. $), 3 \mathbf{a}(1.5$ eq. $),\left[\mathrm{Cp} * \mathrm{RhCl}_{2}\right]_{2}(5$ mol \%), $\operatorname{AgSbF}_{6}(20 \mathrm{~mol} \%)$ in DCM at room temperature under nitrogen atmosphere for $20 \mathrm{~h}$. ${ }^{[\mathrm{b}]}$ Isolated yield.

\section{(4) Typical procedure for the catalytic three-component reaction}

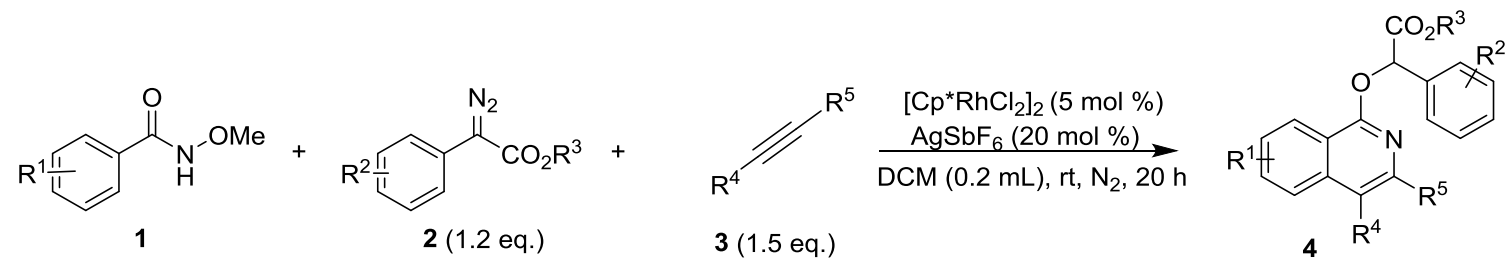

$N$-Methoxybenzamide $1(0.10 \mathrm{mmol})$, alkyne $3(0.15 \mathrm{mmol})$ and $\left[\mathrm{Cp}^{*} \mathrm{RhCl}_{2}\right]_{2}(3.1 \mathrm{mg}$, $0.005 \mathrm{mmol}$ ) were added to an oven-dried reaction vessel equipped with magnetic stirring bar. The silver hexafluoroantimonate $(6.9 \mathrm{mg}, 0.02 \mathrm{mmol})$, anhydrous DCM $(0.2 \mathrm{~mL})$ and $\alpha$-diazoester $2(0.12 \mathrm{mmol})$ were then added under nitrogen atmosphere. The resulting mixture was stirred at room temperature for $20 \mathrm{~h}$. The product 4 was obtained by silica gel column chromatography (petroleum ether/diethylether $=100: 1$ ). 


\section{(5) Large scaled preparation of 4aaa}

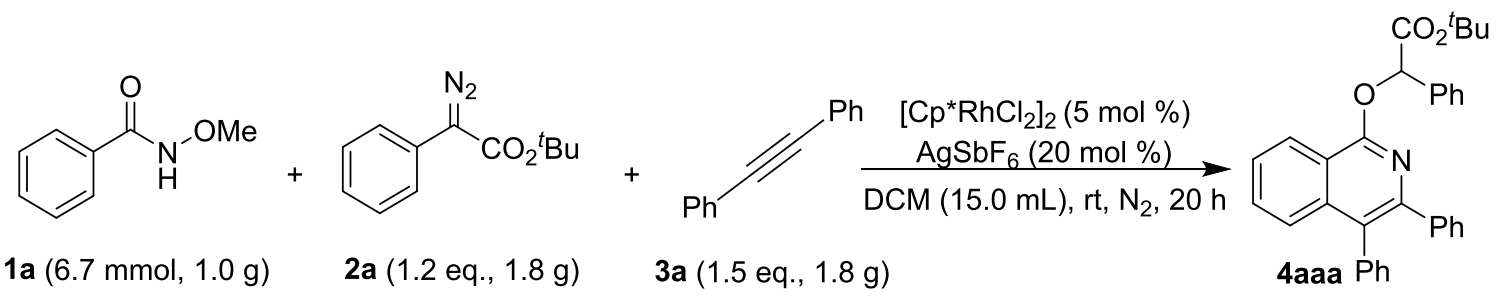

$N$-Methoxybenzamide 1a $(1.0 \mathrm{~g}, 6.70 \mathrm{mmol})$, diphenylacetylene $3 \mathbf{a}(1.8 \mathrm{~g}, 10.05 \mathrm{mmol})$ and $\left[\mathrm{Cp}^{*} \mathrm{RhCl}_{2}\right]_{2}(210.2 \mathrm{mg}, 0.34 \mathrm{mmol})$ were added to an oven-dried reaction vessel equipped with magnetic stirring bar. The silver hexafluoroantimonate $(460.5 \mathrm{mg}, 1.34$ mmol), anhydrous DCM $(15.0 \mathrm{~mL})$ and $\alpha$-diazoester $2 \mathrm{a}(1.8 \mathrm{~g}, 8.04 \mathrm{mmol})$ were then added under nitrogen atmosphere. The resulting mixture was stirred at room temperature for $20 \mathrm{~h}$. The reaction mixture was purified by silica gel column chromatography (petroleum ether/diethylether $=100: 1)$ to give the desired product 4aaa in 83\% yield as a white solid.

\section{(6) Transformation of the product 4aam to confirm its structure}<smiles>CCCC(=O)C(Oc1nc(-c2ccccc2)c(C)c2ccccc12)C(=O)c1ccccc1</smiles>

4aam<smiles>Cc1c(-c2ccccc2)[nH]c(=O)c2ccccc12</smiles>

8, $89 \%$ yield

A dried glass bottle $(10 \mathrm{~mL})$ was charged with 4 aam $(42.5 \mathrm{mg}, 0.1 \mathrm{mmol})$ followed by adding DCM $(0.5 \mathrm{~mL})$ and TFA $(11.1 \mathrm{uL}, 0.15 \mathrm{mmol})$. The reaction mixture was stirred in oil bath at $40{ }^{\circ} \mathrm{C}$ for $3 \mathrm{~h}$. The reaction mixture was concentrated under reduced pressure and purified by silica gel column chromatography (petroleum ether/ ethyl acetate $=2: 1)$ to give the desired product $\mathbf{8}$ in $89 \%$ yield as a white solid. 


\section{(7) Mechanistic studies}

\section{Control experiments (Route 1)}

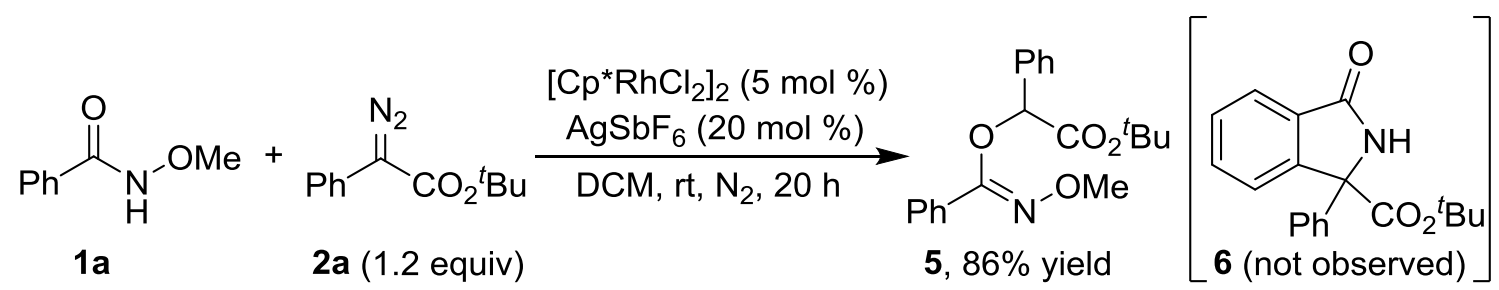<smiles>CO/N=C(\OC(C(=O)OC)c1ccccc1)c1ccccc1</smiles>

5

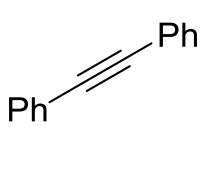

$3 a$ ( 1.5 equiv)

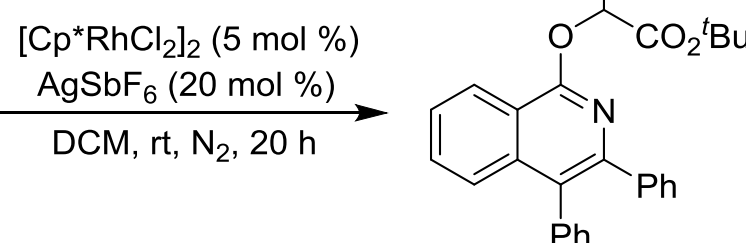

4aaa, $70 \%$ yield

(i) $\mathrm{N}$-Methoxybenzamide $\mathbf{1 a}(15.1 \mathrm{mg}, 0.10 \mathrm{mmol})$ and $\left[\mathrm{Cp}^{*} \mathrm{RhCl}_{2}\right]_{2}(3.1 \mathrm{mg}, 0.005$ mmol) were added in a $15 \mathrm{~mL}$ dried screw-capped tube, followed by the addition of silver hexafluoroantimonate $(6.9 \mathrm{mg}, 0.02 \mathrm{mmol}), \mathrm{DCM}(0.2 \mathrm{~mL})$ and $\alpha$-diazoester $2 \mathbf{a}(26.2 \mathrm{mg}$, $0.12 \mathrm{mmol}$ ) under nitrogen atmosphere. The mixture was stirred at room temperature for $20 \mathrm{~h}$. The reaction mixture was purified by silica gel column chromatography (petroleum ether/diethylether $=100: 1)$ to give the desired product 5 in $86 \%$ yield as a colorless oil. The structure of the compound $\mathbf{5}$ was confirmed by successfully converting it to the compound 10: To a $10 \mathrm{~mL}$ dried glass bottle charged with 5 (34.1 mg, $0.1 \mathrm{mmol})$ were added DCM $(0.5 \mathrm{~mL})$ and TFA $(11.1 \mathrm{uL}, 0.15 \mathrm{mmol})$. The reaction mixture was heated at $40{ }^{\circ} \mathrm{C}$ for $3 \mathrm{~h}$. The solvent was removed under reduced pressure to give the mixture of compounds $1 \mathrm{a}$ and $\mathbf{9}$, which was dissolved in toluene/methanol $\left(\mathrm{V}_{1}: \mathrm{V}_{2}=3: 1\right)$. To the resulting mixture was added dropwise the $\mathrm{TMSCHN}_{2}(22.2 \mathrm{uL}, 0.15 \mathrm{mmol})$ at $0{ }^{\circ} \mathrm{C}$. Then it was warmed to room temperature and stirred for $12 \mathrm{~h}$. The reaction solution was concentrated under reduced pressure and purified by silica gel column chromatography (petroleum ether/diethyl ether $=50: 1$ ), giving the desired product $\mathbf{1 0}$ as a white solid (27.8 $\mathrm{mg}, 56 \%$ yield). The characterization data for the compound $\mathbf{1 0}$ is in accord with reported data, ${ }^{[4]}$ indicating the structure of compound $\mathbf{5}$ is what we have drawn here. 


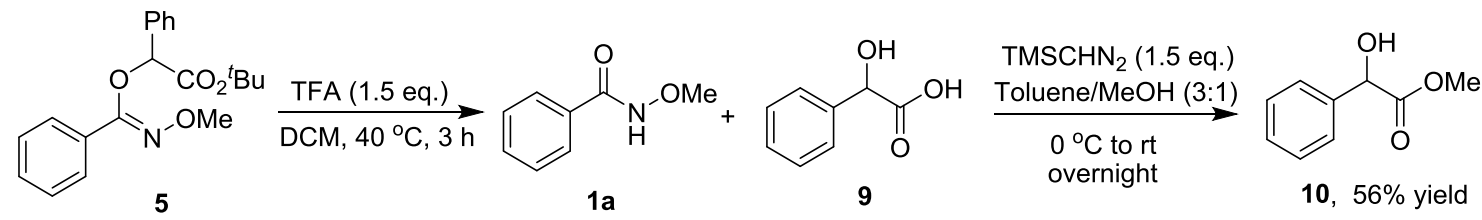

(ii) To an oven-dried $5.0 \mathrm{~mL}$ glass bottle charged with 5 (34.1 mg, $0.1 \mathrm{mmol}), \mathbf{3 a}(26.7$ $\mathrm{mg}, \quad 0.15 \mathrm{mmol})$ and $\left[\mathrm{Cp}^{*} \mathrm{RhCl}_{2}\right]_{2}(3.1 \mathrm{mg}, 0.005 \mathrm{mmol})$ were added silver hexafluoroantimonate $(6.9 \mathrm{mg}, 0.02 \mathrm{mmol})$ and anhydrous DCM $(0.2 \mathrm{~mL})$ under nitrogen atmosphere. The resulting mixture was stirred at room temperature for $20 \mathrm{~h}$. The reaction mixture was purified by silica gel column chromatography (petroleum ether/diethylether $=100: 1)$ to give the desired product 4 aaa in $70 \%$ yield as a white solid.

\section{Control experiments (Route 2)}<smiles>CONC(=O)c1ccccc1</smiles>

$1 \mathrm{a}$<smiles>O=c1[nH]c(-c2ccccc2)c(-c2ccccc2)c2ccccc12</smiles>

7<smiles>C(#Cc1ccccc1)c1ccccc1</smiles>

3a (1.5 equiv)

$\left[\mathrm{Cp}^{*} \mathrm{RhCl}_{2}\right]_{2}(5 \mathrm{~mol} \%)$

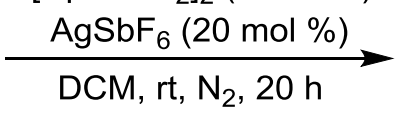

$\left[\mathrm{Cp}^{*} \mathrm{RhCl}_{2}\right]_{2}(5 \mathrm{~mol} \%)$<smiles>N#CC(=O)c1ccccc1</smiles>

2a (1.2 equiv)<smiles>O=c1[nH]c(-c2ccccc2)c(-c2ccccc2)c2ccccc12</smiles>

$7,29 \%$ yield<smiles>CCCCOC(=O)C(Oc1nc(-c2ccccc2)c(-c2ccccc2)c2ccccc12)c1ccccc1</smiles>

4aaa, N.R.

(i) $\mathrm{N}$-Methoxybenzamide $1 \mathbf{a}(15.1 \mathrm{mg}, 0.10 \mathrm{mmol})$, diphenylacetylene 3a $(26.7 \mathrm{mg}, 0.15$ $\mathrm{mmol})$ and $\left[\mathrm{Cp}^{*} \mathrm{RhCl}_{2}\right]_{2}(3.1 \mathrm{mg}, 0.005 \mathrm{mmol})$ were added to an oven-dried reaction vessel. The silver hexafluoroantimonate $(6.9 \mathrm{mg}, 0.02 \mathrm{mmol})$ and anhydrous DCM $(0.2$ $\mathrm{mL}$ ) was then added under nitrogen atmosphere. The resulting mixture was stirred at room temperature for $20 \mathrm{~h}$. The reaction mixture was purified by silica gel column chromatography (petroleum ether/diethylether $=5: 1$ ) to afford the product 7 in $29 \%$ yield as a white solid. 
(ii) To an oven-dried $5.0 \mathrm{~mL}$ glass bottle charged with $7(34.1 \mathrm{mg}, 0.1 \mathrm{mmol})$ and $\left[\mathrm{Cp}^{*} \mathrm{RhCl}_{2}\right]_{2}(3.1 \mathrm{mg}, 0.005 \mathrm{mmol})$ were added silver hexafluoroantimonate $(6.9 \mathrm{mg}$, $0.02 \mathrm{mmol})$, anhydrous DCM $(0.2 \mathrm{~mL})$ and $\alpha$-diazoester $\mathbf{2 a}(26.2 \mathrm{mg}, 0.12 \mathrm{mmol})$ under nitrogen atmosphere. The resulting mixture was stirred at room temperature for $20 \mathrm{~h}$. No reaction was observed.

\section{Monitoring the reaction progress}

Under nitrogen atmosphere, $N$-methoxybenzamide $1 \mathrm{a}(15.1 \mathrm{mg}, 0.1 \mathrm{mmol})$, diphenylacetylene $3 \mathbf{a}(26.7 \mathrm{mg}, 0.15 \mathrm{mmol})$ and $\left[\mathrm{Cp}^{*} \mathrm{RhCl}_{2}\right]_{2}(3.1 \mathrm{mg}, 0.005 \mathrm{mmol})$, $\mathrm{AgSbF}_{6}$ (6.9 mg, $0.02 \mathrm{mmol}$ ), $\mathrm{CH}_{2} \mathrm{Br}_{2}$ (internal standard, $17.4 \mathrm{mg}, 0.1 \mathrm{mmol}$ ), and anhydrous $\mathrm{CD}_{2} \mathrm{Cl}_{2}(0.5 \mathrm{~mL})$ were added to an oven-dried glass vial with stirring. The solution part was transferred to a dry NMR tube, which was immediately subjected to ${ }^{1} \mathrm{H}$ NMR analysis once $\alpha$-diazoester $2 \mathbf{a}(26.2 \mathrm{mg}, 0.12 \mathrm{mmol})$ was added. The amounts of $\mathbf{5}$ and 4aaa were monitored in the real time by ${ }^{1} \mathrm{H}$ NMR spectrometer. The ${ }^{1} \mathrm{H}$ NMR spectra are included in the Copies of ${ }^{1} \mathbf{H}$ and ${ }^{13} \mathrm{C}$ NMR spectra.

\section{Table S8. Monitoring the reaction progress ${ }^{[\mathrm{a}]}$}

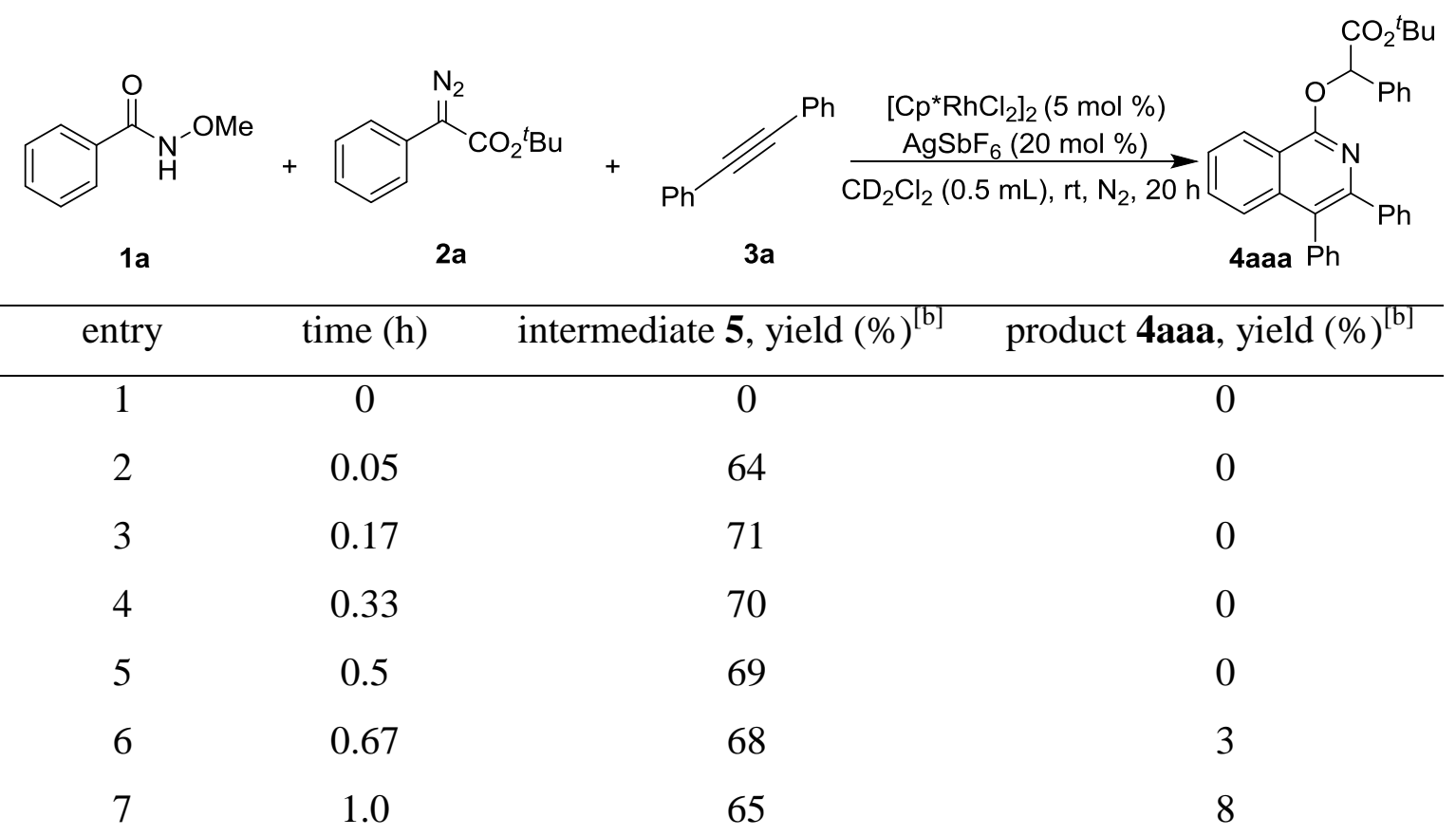




$\begin{array}{llll}8 & 1.67 & 53 & 17 \\ 9 & 3.5 & 41 & 26 \\ 10 & 5.0 & 31 & 34 \\ 11 & 5.5 & 25 & 39 \\ 12 & 7.5 & 20 & 44 \\ 13 & 19 & 12 & 53\end{array}$

${ }^{\text {aa }}$ The reaction was carried out with 1a $(0.10 \mathrm{mmol}), \mathbf{2 a}(1.2 \mathrm{eq}),. \mathbf{3 a}(1.5 \mathrm{eq}),.\left[\mathrm{Cp} * \mathrm{RhCl}_{2}\right]_{2}(5 \mathrm{~mol} \%)$, $\mathrm{AgSbF}_{6}(20 \mathrm{~mol} \%)$ in $\mathrm{CD}_{2} \mathrm{Cl}_{2}$ at room temperature under nitrogen atmosphere with $\mathrm{CH}_{2} \mathrm{Br}_{2}$ as the internal standard. ${ }^{[b]}$ Determined by integration of ${ }^{1} \mathrm{H}$ NMR spectra.

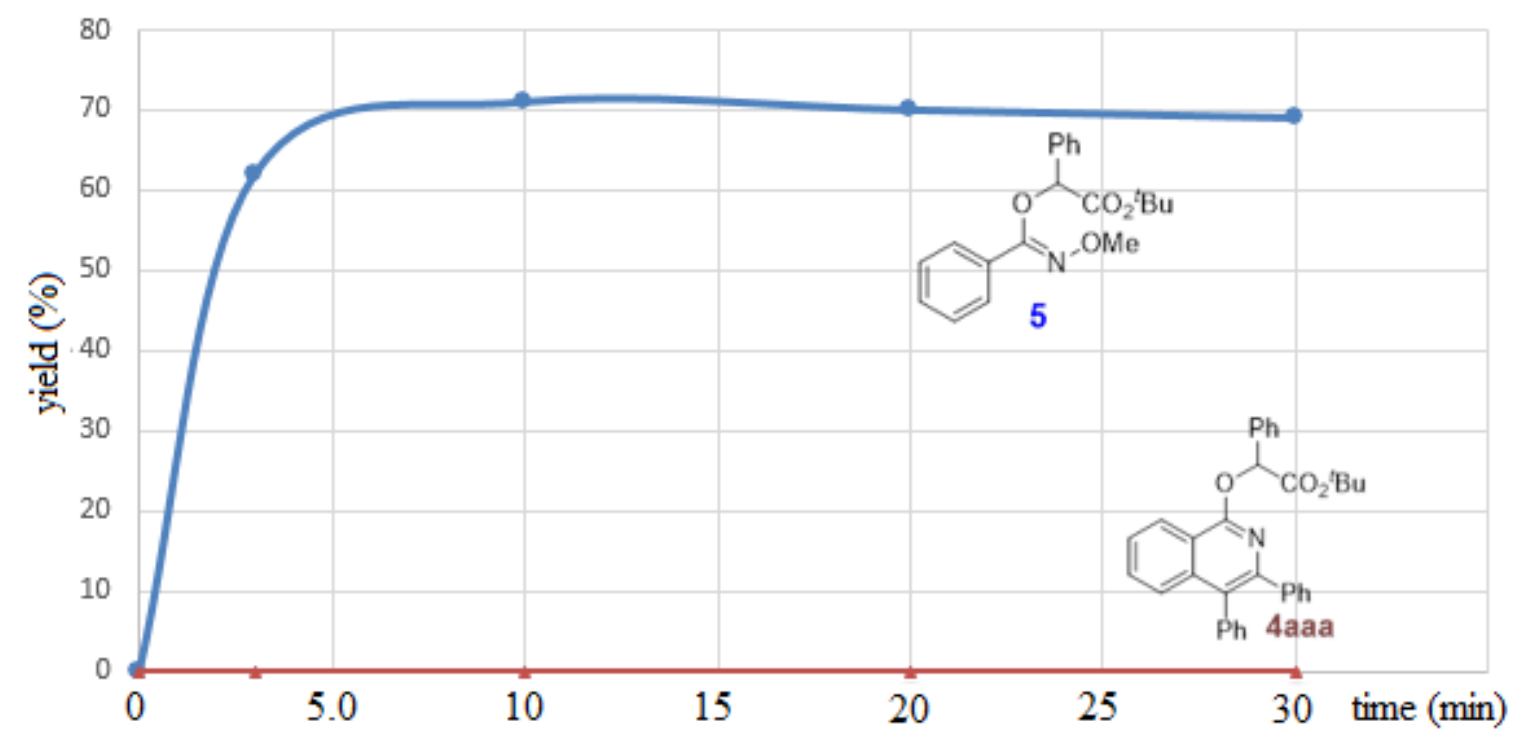

(a)

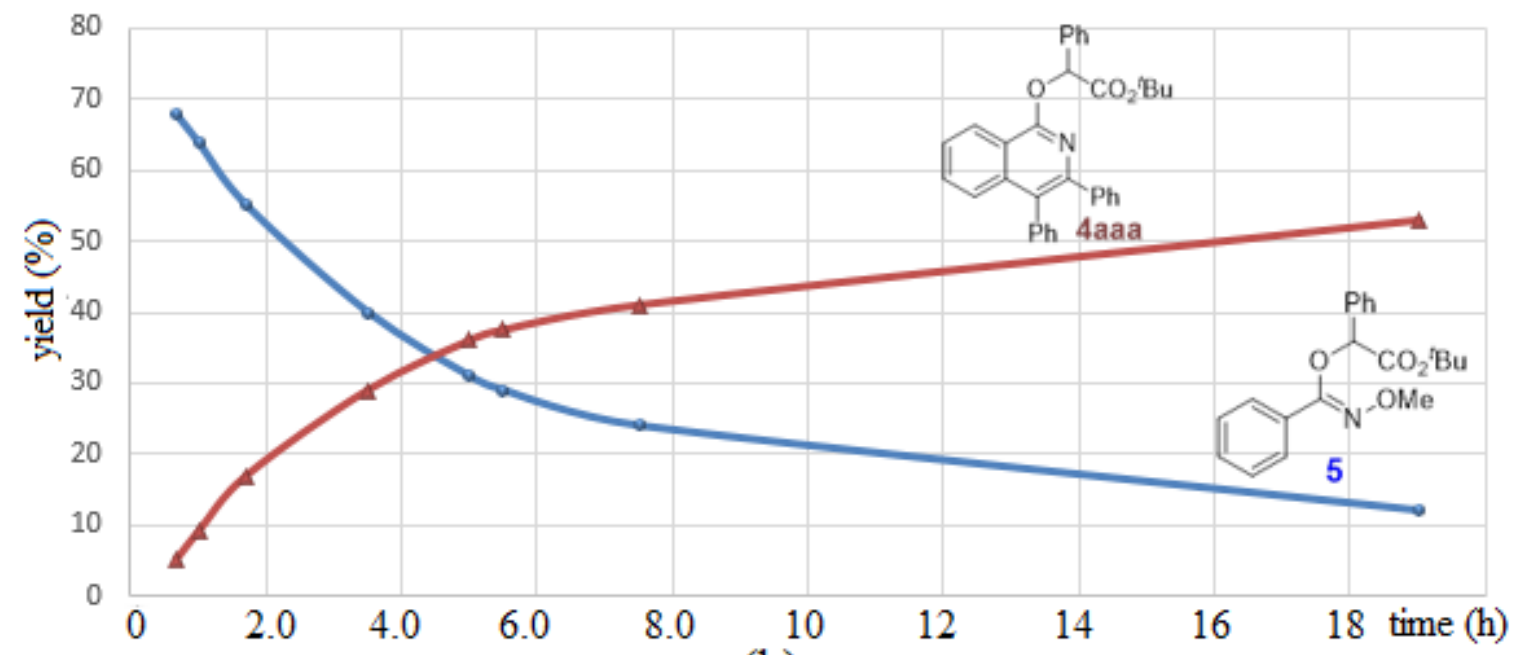

(b) 


\section{H/D exchange experiment}

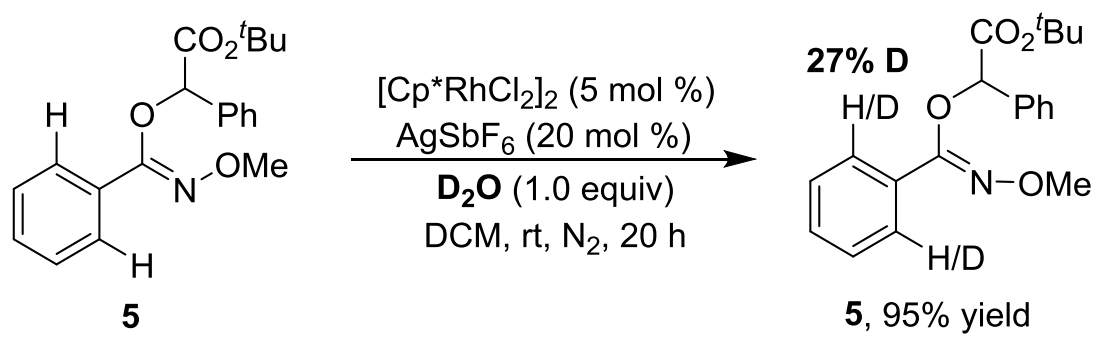

To an oven-dried $5.0 \mathrm{~mL}$ glass bottle charged with $5(34.1 \mathrm{mg}, 0.1 \mathrm{mmol})$ and $\left[\mathrm{Cp}^{*} \mathrm{RhCl}_{2}\right]_{2}(3.1 \mathrm{mg}, 0.005 \mathrm{mmol})$ was added silver hexafluoroantimonate $(6.9 \mathrm{mg}, 0.02$ mmol), anhydrous DCM $(0.2 \mathrm{~mL})$ and $\mathrm{D}_{2} \mathrm{O}(1.8 \mathrm{uL}, 0.1 \mathrm{mmol})$ under nitrogen atmosphere. The resulting mixture was stirred at room temperature for $20 \mathrm{~h}$. The reaction mixture was purified by silica gel column chromatography (petroleum ether/diethylether = 100:1) to afford the product $\mathbf{5}$ (32.4 mg, 95\% yield). The deuterium incorporation in $\mathbf{5}$ was determined by ${ }^{1} \mathrm{H}$ NMR analysis.

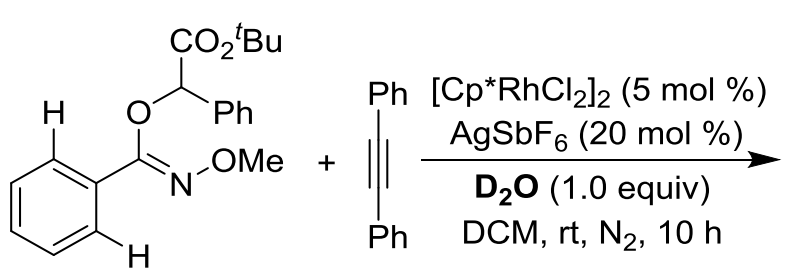

5

$3 \mathbf{a}(1.5$ equiv)<smiles>CC(C)(C)OC(=O)C(Oc1nc(-c2ccccc2)c(-c2ccccc2)c2cccc([18OH])c12)c1ccccc1</smiles>

4aaa, $58 \%$ yield<smiles></smiles>

5, $17 \%$ yield

To an oven-dried $5.0 \mathrm{~mL}$ glass bottle charged with $5(34.1 \mathrm{mg}, 0.1 \mathrm{mmol}), \mathbf{3 a}(26.7 \mathrm{mg}$, $0.15 \mathrm{mmol})$ and $\left[\mathrm{Cp}^{*} \mathrm{RhCl}_{2}\right]_{2} \quad(3.1 \mathrm{mg}, \quad 0.005 \mathrm{mmol})$ were added silver hexafluoroantimonate $(6.9 \mathrm{mg}, 0.02 \mathrm{mmol})$, anhydrous DCM $(0.2 \mathrm{~mL})$ and $\mathrm{D}_{2} \mathrm{O}(1.8 \mathrm{uL}$, $0.1 \mathrm{mmol}$ ) under nitrogen atmosphere. The resulting mixture was stirred at room temperature for $20 \mathrm{~h}$. The reaction mixture was purified by silica gel column chromatography (petroleum ether/diethylether $=100: 1)$ to afford the product 4aaa $(28.3$ $\mathrm{mg}, 58 \%$ yield) and recovered $5(5.8 \mathrm{mg}, 17 \%$ yield). The deuterium incorporation in 4aaa and 5 was determined by ${ }^{1} \mathrm{H}$ NMR analysis. 


\section{(C) Characterization Data}

tert-butyl 2-((3,4-diphenylisoquinolin-1-yl)oxy)-2-phenylacetate (4aaa)

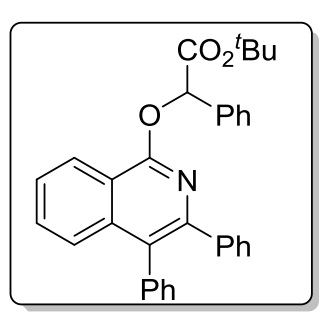

White solid; $39.5 \mathrm{mg}, 81 \%$ yield; ${ }^{1} \mathbf{H}$ NMR (400 $\mathbf{M H z}, \mathbf{C D C l}_{3}$ ): $\delta=$ $8.46(\mathrm{~d}, J=7.1 \mathrm{~Hz}, 1 \mathrm{H}), 7.84-7.70(\mathrm{~m}, 2 \mathrm{H}), 7.58-7.50(\mathrm{~m}, 3 \mathrm{H})$, $7.49-7.40$ (m, 5H), $7.38-7.30(\mathrm{~m}, 3 \mathrm{H}), 7.28-7.22(\mathrm{~m}, 2 \mathrm{H}), 7.22$ - 7.13 (m, 3H), 6.39 (s, 1H), 1.29 (s, 9H). ${ }^{\mathbf{1 3}} \mathbf{C}$ NMR (100 MHz, $\left.\mathbf{C D C l}_{3}\right): \delta=169.34,157.96,146.27,140.51,138.77,137.86$, $135.89,131.71,131.68,130.65,130.53,128.70,128.65,128.46,128.42,127.72,127.34$, $127.17,127.01,126.41,125.66,125.54,124.09,118.10,81.80,76.53,27.85$. HRMS (ESI): $[\mathrm{M}+\mathrm{H}]^{+}$calculated for $\mathrm{C}_{33} \mathrm{H}_{30} \mathrm{NO}_{3}{ }^{+}: 488.2220$, found: 488.2221 .

tert-butyl2-((6-methyl-3,4-diphenylisoquinolin-1-yl)oxy)-2-phenylacetate (4baa)

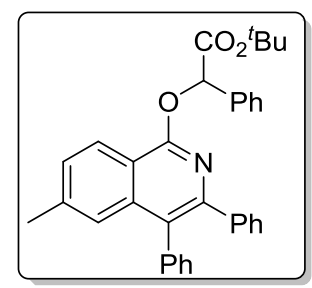

White solid; $39.1 \mathrm{mg}, 78 \%$ yield; ${ }^{1} \mathbf{H}$ NMR (400 $\left.\mathbf{M H z}, \mathbf{C D C l}_{3}\right): \delta=$ $8.35(\mathrm{~d}, J=8.3 \mathrm{~Hz}, 1 \mathrm{H}), 7.79-7.69(\mathrm{~m}, 2 \mathrm{H}), 7.47-7.37(\mathrm{~m}, 5 \mathrm{H})$, $7.37-7.29$ (m, 5H), $7.26-7.21(\mathrm{~m}, 2 \mathrm{H}), 7.19-7.10(\mathrm{~m}, 3 \mathrm{H}), 6.38$ (s, 1H), 2.38 (s, 3H), 1.29 (s, 9H). ${ }^{13} \mathbf{C}$ NMR (100 MHz, $\left.\mathbf{C D C l}_{3}\right): \delta$ $=169.37,157.96,146.43,140.93,140.67,139.00,138.03,135.98$,

$131.74,131.71,130.51,128.64,128.61,128.43,128.38,127.71,127.28,127.07,126.90$, $125.28,124.65,123.97,116.27,81.69,76.46,27.84,22.28$. HRMS (ESI): $[\mathrm{M}+\mathrm{H}]^{+}$ calculated for $\mathrm{C}_{34} \mathrm{H}_{32} \mathrm{NO}_{3}{ }^{+}$: 502.2377, found: 502.2378 .

tert-butyl 2-((6-fluoro-3,4-diphenylisoquinolin-1-yl)oxy)-2-phenylacetate (4caa)

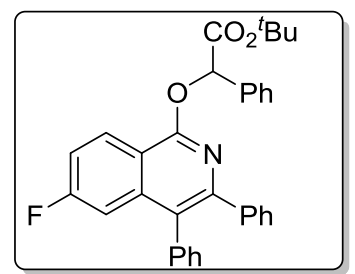

White solid; $35.4 \mathrm{mg}, 70 \%$ yield; ${ }^{1} \mathbf{H}$ NMR (400 $\left.\mathbf{~ M H z}, \mathbf{C D C l}_{3}\right): \delta$ $=8.54-8.39(\mathrm{~m}, 1 \mathrm{H}), 7.77-7.68(\mathrm{~m}, 2 \mathrm{H}), 7.49-7.44(\mathrm{~m}, 2 \mathrm{H})$, $7.43-7.40(\mathrm{~m}, 2 \mathrm{H}), 7.40-7.31(\mathrm{~m}, 4 \mathrm{H}), 7.31-7.21(\mathrm{~m}, 3 \mathrm{H}), 7.21$ - $7.11(\mathrm{~m}, 4 \mathrm{H}), 6.37$ (s, 1H), 1.29 (s, 9H). ${ }^{19} \mathbf{F}$ NMR (376 MHz, $\left.\mathbf{C D C l}_{\mathbf{3}}\right): \delta=-106.79 .{ }^{13} \mathbf{C}$ NMR (100 MHz, $\left.\mathbf{C D C l}_{\mathbf{3}}\right): \delta=169.20,164.07(\mathrm{~d}, J=250.4$ $\mathrm{Hz}), 157.84,147.63,140.92(\mathrm{~d}, J=10.0 \mathrm{~Hz}), 140.19,137.40,135.70,131.53,131.49$, $130.50,128.82,128.71,128.68,128.63,127.75,127.45,127.40,127.28,127.25(\mathrm{~d}, J=$ $10.5 \mathrm{~Hz}), 125.38(\mathrm{~d}, J=4.9 \mathrm{~Hz}), 116.22(\mathrm{~d}, J=25.1 \mathrm{~Hz}), 115.08,109.74(\mathrm{~d}, J=22.8 \mathrm{~Hz})$, 81.93, 76.62, 27.84. HRMS (ESI): $[\mathrm{M}+\mathrm{H}]^{+}$calculated for $\mathrm{C}_{33} \mathrm{H}_{29} \mathrm{FNO}_{3}{ }^{+}$: 506.2126, 
found: 506.2129 .

tert-butyl 2-((6-chloro-3,4-diphenylisoquinolin-1-yl)oxy)-2-phenylacetate (4daa)

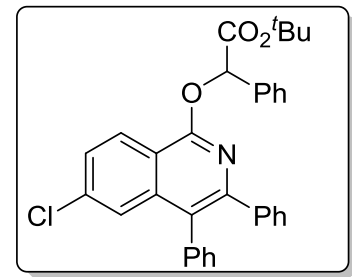

White solid; $37.0 \mathrm{mg}, 71 \%$ yield; ${ }^{1} \mathbf{H}$ NMR (400 $\mathbf{M H z}, \mathbf{C D C l}_{3}$ ): $\delta$ $=8.39(\mathrm{~d}, J=8.8 \mathrm{~Hz}, 1 \mathrm{H}), 7.79-7.68(\mathrm{~m}, 2 \mathrm{H}), 7.55-7.51(\mathrm{~m}$, $1 \mathrm{H}), 7.50-7.44(\mathrm{~m}, 3 \mathrm{H}), 7.44-7.41(\mathrm{~m}, 1 \mathrm{H}), 7.41-7.35(\mathrm{~m}, 5 \mathrm{H})$, $7.26-7.20(\mathrm{~m}, 2 \mathrm{H}), 7.20-7.14(\mathrm{~m}, 3 \mathrm{H}), 6.36(\mathrm{~s}, 1 \mathrm{H}), 1.29(\mathrm{~s}, 9 \mathrm{H})$.

${ }^{13} \mathbf{C}$ NMR (100 MHz, $\left.\mathbf{C D C l}_{3}\right): \delta=169.14,157.91,147.78,140.15,139.91,137.28$, 137.14 , 135.66, 131.61, 131.57, 130.49, 128.84, 128.72, 128.70, 128.66, 127.76, 127.51, $127.42,127.31,127.27,125.97,124.93,124.65,116.36,81.98,76.69,27.85$. HRMS (ESI): $[\mathrm{M}+\mathrm{H}]^{+}$calculated for $\mathrm{C}_{33} \mathrm{H}_{29}{ }^{35} \mathrm{ClNO}_{3}{ }^{+}: 522.1830$, found: 522.1833 .

tert-butyl 2-((6-bromo-3,4-diphenylisoquinolin-1-yl)oxy)-2-phenylacetate (4eaa)

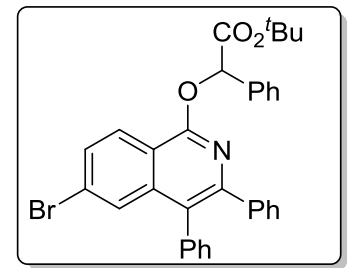

White solid; $41.8 \mathrm{mg}, 74 \%$ yield; ${ }^{1} \mathbf{H}$ NMR (400 $\mathbf{M H z}, \mathbf{C D C l}_{\mathbf{3}}$ ): $\delta=8.31(\mathrm{~d}, J=8.8 \mathrm{~Hz}, 1 \mathrm{H}), 7.76-7.68(\mathrm{~m}, 3 \mathrm{H}), 7.66-7.56(\mathrm{~m}$, $1 \mathrm{H}), 7.48-7.41(\mathrm{~m}, 3 \mathrm{H}), 7.41-7.33(\mathrm{~m}, 6 \mathrm{H}), 7.26-7.20(\mathrm{~m}, 2 \mathrm{H})$, $7.19-7.14$ (m, 2H), 6.36 (s, 1H), 1.29 (s, 9H). ${ }^{13}$ C NMR (100

MHz, $\left.\mathbf{C D C l}_{3}\right): \delta=169.09,157.98,147.76,140.13,137.06,135.62,131.84,131.59$, 131.56, 130.48, 129.86, 128.83, 128.75, 128.70, 128.65, 127.87, 127.74, 127.51, 127.40, 127.30, 125.98, 125.93, 124.77, 116.57, 81.94, 76.69, 27.84. HRMS (ESI): $[\mathrm{M}+\mathrm{H}]^{+}$ calculated for $\mathrm{C}_{33} \mathrm{H}_{29}{ }^{79} \mathrm{BrNO}_{3}{ }^{+}: 566.1325$, found: 566.1312 .

tert-butyl 2-((6-(tert-butyl)-3,4-diphenylisoquinolin-1-yl)oxy)-2-phenylacetate (4faa)

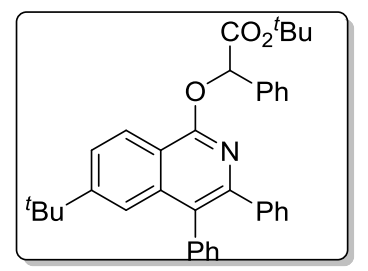

White solid; 40.7 mg, 75\% yield; ${ }^{1} \mathbf{H}$ NMR (400 MHz, $\mathbf{C D C l}_{3}$ ): $\delta=8.40(\mathrm{~d}, J=8.7 \mathrm{~Hz}, 1 \mathrm{H}), 7.77-7.74(\mathrm{~m}, 2 \mathrm{H}), 7.62(\mathrm{dd}, J=8.7$, $1.8 \mathrm{~Hz}, 1 \mathrm{H}), 7.54-7.53(\mathrm{~m}, 1 \mathrm{H}) 7.48-7.39$ (m, 5H), $7.38-7.31$ $(\mathrm{m}, 3 \mathrm{H}), 7.27-7.24(\mathrm{~m}, 2 \mathrm{H}), 7.19-7.15(\mathrm{~m}, 3 \mathrm{H}), 6.40(\mathrm{~s}, 1 \mathrm{H})$, 1.30 (s, 9H), 1.26 (s, 9H). ${ }^{13} \mathbf{C}$ NMR (100 MHz, $\left.\mathbf{C D C l}_{3}\right): \delta=169.41,157.85,153.74$, 146.33 , 140.77, 138.81, 138.03, 136.05, 131.72, 130.55, 128.62, 128.36, 128.30, 127.70, $127.31,127.08,126.89,125.87,125.10,123.76,120.85,116.23,81.71,76.39,35.35$, 31.06, 27.85. HRMS (ESI): $[\mathrm{M}+\mathrm{H}]^{+}$calculated for $\mathrm{C}_{37} \mathrm{H}_{38} \mathrm{NO}_{3}{ }^{+}:$544.2846, found: 544.2846 . 
tert-butyl 2-phenyl-2-((3,4,6-triphenylisoquinolin-1-yl)oxy)acetate (4gaa)

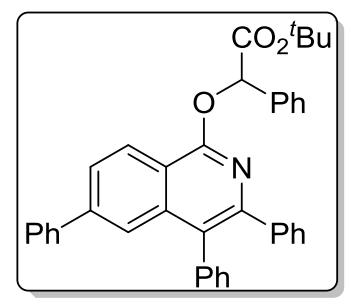

White solid; $40.0 \mathrm{mg}, 71 \%$ yield; ${ }^{\mathbf{1}} \mathbf{H}$ NMR (400 $\mathbf{M H z}, \mathbf{C D C l}_{\mathbf{3}}$ ): $\delta=8.57-8.46(\mathrm{~m}, 1 \mathrm{H}), 7.81-7.74(\mathrm{~m}, 4 \mathrm{H}), 7.57-7.51(\mathrm{~m}, 2 \mathrm{H})$, $7.50-7.44$ (m, 2H), $7.44-7.40(\mathrm{~m}, 4 \mathrm{H}), 7.39-7.36(\mathrm{~m}, 2 \mathrm{H}), 7.36$ $-7.30(\mathrm{~m}, 3 \mathrm{H}), 7.30-7.25(\mathrm{~m}, 2 \mathrm{H}), 7.20-7.14(\mathrm{~m}, 3 \mathrm{H}), 6.41(\mathrm{~s}$, 1H), $1.30(\mathrm{~s}, 9 \mathrm{H}) .{ }^{13} \mathbf{C}$ NMR (100 MHz, $\left.\mathbf{C D C l}_{3}\right): \delta=169.33$, 158.02, 146.89, 143.36, 140.81, 140.56, 139.15, 137.78, 135.92, 131.75, 131.72, 130.55, $128.93,128.73,128.68,128.54,128.49,127.93,127.76,127.64,127.36,127.25,127.05$, 126.07, 125.89, 124.72, 123.55, 117.13, 81.82, 76.57, 27.87. HRMS (ESI): $[\mathrm{M}+\mathrm{H}]^{+}$ calculated for $\mathrm{C}_{39} \mathrm{H}_{34} \mathrm{NO}_{3}{ }^{+}: 564.2533$, found: 564.2532 .

tert-butyl 2-((8-methyl-3,4-diphenylisoquinolin-1-yl)oxy)-2-phenylacetate (4haa)

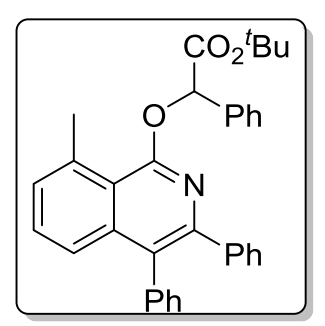

White solid; $31.1 \mathrm{mg}, 62 \%$ yield; ${ }^{1} \mathbf{H}$ NMR (400 MHz, $\mathbf{C D C l}_{3}$ ): $\delta=$ $7.74-7.67(\mathrm{~m}, 1 \mathrm{H}), 7.45-7.39(\mathrm{~m}, 4 \mathrm{H}), 7.38-7.22(\mathrm{~m}, 8 \mathrm{H}), 7.22-$ $7.10(\mathrm{~m}, 4 \mathrm{H}), 6.41(\mathrm{~s}, 1 \mathrm{H}), 3.02(\mathrm{~s}, 3 \mathrm{H}), 1.26(\mathrm{~s}, 9 \mathrm{H}) .{ }^{13} \mathbf{C}$ NMR (100 MHz, $\left.\mathbf{C D C l}_{3}\right): \delta=169.55,158.86,145.69,140.66,140.43,138.50$, $136.84,135.96,131.86,131.71,130.46,129.94,129.35,128.63$, 128.59 , 128.48, 128.40, 128.14, 127.34, 127.12, 126.96, 126.10, 123.95, 117.94, 81.66, 77.35, 27.81, 25.18. HRMS (ESI): $[\mathrm{M}+\mathrm{H}]^{+}$calculated for $\mathrm{C}_{34} \mathrm{H}_{32} \mathrm{NO}_{3}{ }^{+}:$502.2377, found: 502.2374 .

tert-butyl 2-((7-methyl-3,4-diphenylisoquinolin-1-yl)oxy)-2-phenylacetate (4iaa)

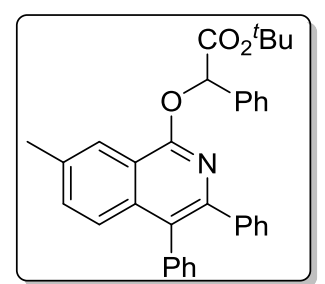

White solid; $32.1 \mathrm{mg}, 64 \%$ yield; ${ }^{1} \mathbf{H}$ NMR (400 $\mathbf{M H z}, \mathbf{C D C l}_{3}$ ): $\delta=$ $8.24-8.18(\mathrm{~m}, 1 \mathrm{H}), 7.81-7.71(\mathrm{~m}, 2 \mathrm{H}), 7.50-7.44(\mathrm{~m}, 3 \mathrm{H}), 7.43-$ $7.38(\mathrm{~m}, 4 \mathrm{H}), 7.38-7.30(\mathrm{~m}, 3 \mathrm{H}), 7.27-7.21(\mathrm{~m}, 2 \mathrm{H}), 7.20-7.13$ $(\mathrm{m}, 3 \mathrm{H}), 6.40(\mathrm{~s}, 1 \mathrm{H}), 2.52(\mathrm{~s}, 3 \mathrm{H}), 1.30(\mathrm{~s}, 9 \mathrm{H}) .{ }^{13} \mathrm{C}$ NMR (100 $\left.\mathbf{M H z}, \mathbf{C D C l}_{3}\right): \delta=169.44,157.60,145.32,140.62,138.09,136.97,136.45,136.03$, 132.72 , 131.70, 131.68, 130.53, 128.70, 128.67, 128.43, 128.39, 127.84, 127.33, 127.11, 126.89, 125.62, 125.50, 123.06, 118.20, 81.78, 76.46, 27.87, 21.72. HRMS (ESI): $[\mathrm{M}+\mathrm{H}]^{+}$calculated for $\mathrm{C}_{34} \mathrm{H}_{32} \mathrm{NO}_{3}{ }^{+}: 502.2377$, found: 502.2379 . 
tert-butyl 2-((3,4-diphenylisoquinolin-1-yl)oxy)-2-(p-tolyl)acetate (4aba)

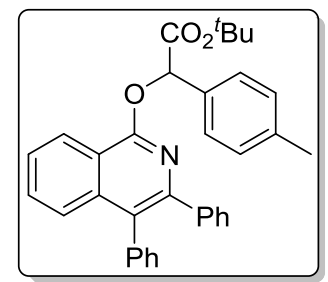

White solid; $25.6 \mathrm{mg}, 51 \%$ yield; ${ }^{1} \mathbf{H}$ NMR (400 $\mathbf{M H z}, \mathbf{C D C l}_{3}$ ): $\delta=$ $8.46-8.43(\mathrm{~m}, 1 \mathrm{H}), 7.67-7.59(\mathrm{~m}, 2 \mathrm{H}), 7.58-7.49(\mathrm{~m}, 3 \mathrm{H}), 7.45$

- $7.40(\mathrm{~m}, 2 \mathrm{H}), 7.38-7.30(\mathrm{~m}, 3 \mathrm{H}), 7.29-7.22(\mathrm{~m}, 4 \mathrm{H}), 7.20-$ $7.14(\mathrm{~m}, 3 \mathrm{H}), 6.35$ (s, 1H), $2.41(\mathrm{~s}, 3 \mathrm{H}), 1.29$ (s, 9H). ${ }^{13} \mathbf{C}$ NMR $\left(\mathbf{1 0 0} \mathbf{~ M H z}, \mathbf{C D C l}_{\mathbf{3}}\right): \delta=169.52,158.08,146.30,140.57,138.78,138.55,137.94,132.95$, $131.73,131.71,130.62$, 130.56, 129.39, 128.47, 128.42, 127.77, 127.35, 127.18, 127.01, 126.38, 125.59, 125.53, 124.18, 118.14, 81.69, 76.43, 27.87, 21.40. HRMS (ESI): $[\mathrm{M}+\mathrm{H}]^{+}$calculated for $\mathrm{C}_{34} \mathrm{H}_{32} \mathrm{NO}_{3}{ }^{+}:$502.2377, found: 502.2380 .

tert-butyl 2-((3,4-diphenylisoquinolin-1-yl)oxy)-2-(4-fluorophenyl)acetate (4aca)

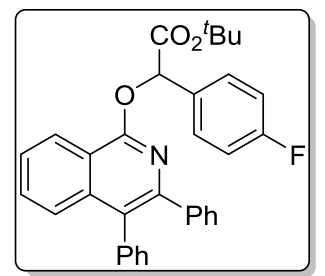

White solid; $28.8 \mathrm{mg}, 57 \%$ yield; ${ }^{1} \mathbf{H}$ NMR (400 $\left.\mathbf{M H z}, \mathbf{C D C l}_{3}\right): \delta=$ $8.45-8.41(\mathrm{~m}, 1 \mathrm{H}), 7.77-7.70(\mathrm{~m}, 2 \mathrm{H}), 7.59-7.51(\mathrm{~m}, 3 \mathrm{H}), 7.44-$ $7.30(\mathrm{~m}, 5 \mathrm{H}), 7.27-7.22(\mathrm{~m}, 2 \mathrm{H}), 7.21-7.10(\mathrm{~m}, 5 \mathrm{H}), 6.37(\mathrm{~s}, 1 \mathrm{H})$, 1.29 (s, 9H). ${ }^{19}$ F NMR (376 $\left.\mathbf{~ M H z}, \mathbf{C D C l}_{3}\right): \delta=-113.09 .{ }^{13} \mathbf{C}$ NMR (100 MHz, $\left.\mathbf{C D C l}_{3}\right): \delta=169.22,163.06$ (d, $\left.J=247.1 \mathrm{~Hz}\right), 157.84,146.29,140.48$, $138.81,137.81,131.81(\mathrm{~d}, J=3.1 \mathrm{~Hz}), 131.71,131.68,130.72,130.51,129.54$ (d, $J=8.3$ Hz), 128.48, 128.44, 127.39, 127.23, 127.07, 126.50, 125.81, 125.63, 124.00, 118.04, $115.66(\mathrm{~d}, J=21.7 \mathrm{~Hz}), 81.99,75.83,27.84$. HRMS (ESI): $[\mathrm{M}+\mathrm{H}]^{+}$calculated for $\mathrm{C}_{33} \mathrm{H}_{29} \mathrm{FNO}_{3}^{+}$: 506.2126, found: 506.2125.

tert-butyl 2-(4-chlorophenyl)-2-((3,4-diphenylisoquinolin-1-yl)oxy)acetate (4ada)

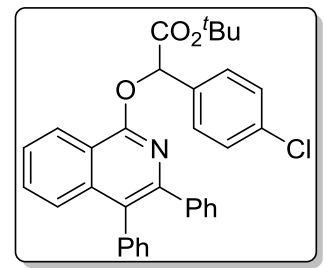

White solid; $31.8 \mathrm{mg}, 61 \%$ yield; ${ }^{1} \mathbf{H}$ NMR (400 $\mathbf{M H z}, \mathbf{C D C l}_{3}$ ): $\delta=$ $8.48-8.38(\mathrm{~m}, 1 \mathrm{H}), 7.73-7.66(\mathrm{~m}, 2 \mathrm{H}), 7.62-7.52(\mathrm{~m}, 3 \mathrm{H}), 7.48$ - $7.43(\mathrm{~m}, 2 \mathrm{H}), 7.42-7.32(\mathrm{~m}, 6 \mathrm{H}), 7.24-7.21(\mathrm{~m}, 1 \mathrm{H}), 7.20-$ $7.13(\mathrm{~m}, 3 \mathrm{H}), 6.37$ (s, 1H), 1.29 (s, 9H). ${ }^{13}$ C NMR (100 MHz, $\left.\mathbf{C D C l}_{3}\right): \delta=168.98,157.75,146.28,140.46,138.83,137.78,134.67,134.51,131.70$, $131.67,130.74,130.50,129.85,129.07,128.91$, 128.48, 128.46, 127.38, 127.23, 127.07, 126.52, 125.64, 123.97, 118.03, 82.13, 75.81, 27.84. HRMS (ESI): $[\mathrm{M}+\mathrm{H}]^{+}$calculated for $\mathrm{C}_{33} \mathrm{H}_{29}{ }^{35} \mathrm{ClNO}_{3}{ }^{+}:$522.1830, found: 522.1832 . 
tert-butyl 2-(4-bromophenyl)-2-((3,4-diphenylisoquinolin-1-yl)oxy)acetate (4aea)

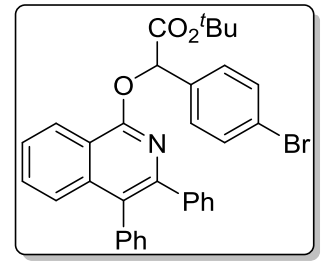

White solid; $35.0 \mathrm{mg}, 62 \%$ yield; ${ }^{1} \mathbf{H}$ NMR (400 $\mathbf{M H z}, \mathbf{C D C l}_{3}$ ): $\delta=$ $8.48-8.36(\mathrm{~m}, 1 \mathrm{H}), 7.73-7.66(\mathrm{~m}, 2 \mathrm{H}), 7.60-7.50(\mathrm{~m}, 3 \mathrm{H}), 7.46-$ $7.32(\mathrm{~m}, 7 \mathrm{H}), 7.28-7.22(\mathrm{~m}, 2 \mathrm{H}), 7.21-7.10(\mathrm{~m}, 3 \mathrm{H}), 6.37(\mathrm{~s}, 1 \mathrm{H})$, 1.30 (s, 9H). ${ }^{13} \mathbf{C}$ NMR (100 MHz, $\left.\mathbf{C D C l}_{3}\right): \delta=168.95,157.72$, $146.25,140.43$, 138.79, 137.75, 134.65, 134.47, 131.68, 131.65, 130.73, 130.49, 129.06, 128.90, 128.47, 128.45, 127.37, 127.22, 127.06, 126.51, 125.84, 125.62, 123.94, 117.99, 82.10, 75.79, 27.82. HRMS (ESI): $[\mathrm{M}+\mathrm{H}]^{+}$calculated for $\mathrm{C}_{33} \mathrm{H}_{29}{ }^{79} \mathrm{BrNO}_{3}{ }^{+}:$566.1325, found: 566.1330 .

tert-butyl 2-((3,4-diphenylisoquinolin-1-yl)oxy)-2-(2-fluorophenyl)acetate (4afa)

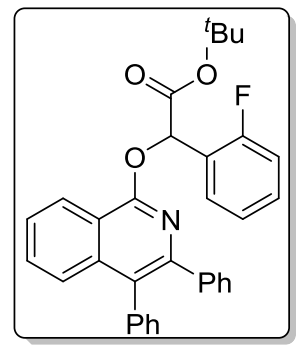

White solid; $39.4 \mathrm{mg}, 78 \%$ yield; ${ }^{1} \mathbf{H}$ NMR (400 $\left.\mathbf{M H z}, \mathbf{C D C l}_{3}\right): \delta=$ $8.41-8.39$ (m, 1H), $7.84-7.79(\mathrm{~m}, 1 \mathrm{H}), 7.55-7.48$ (m, 3H), $7.42-$ $7.32(\mathrm{~m}, 6 \mathrm{H}), 7.28-7.20(\mathrm{~m}, 3 \mathrm{H}), 7.26-7.13(\mathrm{~m}, 4 \mathrm{H}), 6.78(\mathrm{~s}, 1 \mathrm{H})$, $1.32(\mathrm{~s}, 9 \mathrm{H}) .{ }^{19} \mathbf{F}$ NMR (376 MHz, $\left.\mathbf{C D C l}_{3}\right): \delta=-116.70 .{ }^{13} \mathbf{C}$ NMR (100 MHz, $\left.\mathbf{C D C l}_{3}\right): \delta=168.74,160.77$ (d, $\left.J=249.9 \mathrm{~Hz}\right), 157.83$, $146.31,140.49$, 138.80, 137.88, 131.71, 131.67, 130.60, 130.55 (d, $J=6.8 \mathrm{~Hz}$ ), 130.56, 129.63 (d, $J=3.2 \mathrm{~Hz}), 128.45,127.34,127.18$, 127.01, 126.41, 125.78, 125.56, 124.44 (d, $J=3.8 \mathrm{~Hz}), 124.01,123.55(\mathrm{~d}, J=14.1 \mathrm{~Hz}), 118.02,115.79(\mathrm{~d}, J=21.7 \mathrm{~Hz}), 82.03$, $69.93(\mathrm{~d}, J=3.0 \mathrm{~Hz}), 27.83$. HRMS (ESI): $[\mathrm{M}+\mathrm{H}]^{+}$calculated for $\mathrm{C}_{33} \mathrm{H}_{29} \mathrm{FNO}_{3}{ }^{+}$: 506.2126, found: 506.2127 .

tert-butyl 2-(2-chlorophenyl)-2-((3,4-diphenylisoquinolin-1-yl)oxy)acetate (4aga)

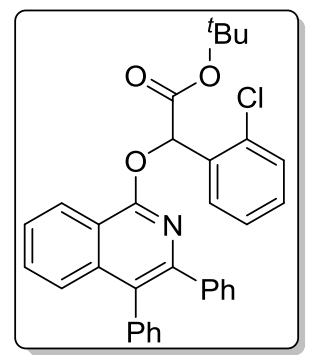

White solid; $24.5 \mathrm{mg}, 47 \%$ yield; ${ }^{1} \mathbf{H}$ NMR (400 $\mathbf{M H z}, \mathbf{C D C l}_{3}$ ): $\delta=$ $8.50-8.36(\mathrm{~m}, 1 \mathrm{H}), 7.72-7.67(\mathrm{~m}, 2 \mathrm{H}), 7.58-7.51(\mathrm{~m}, 3 \mathrm{H}), 7.45-$ $7.42(\mathrm{~m}, 2 \mathrm{H}), 7.41-7.38(\mathrm{~m}, 2 \mathrm{H}), 7.38-7.32(\mathrm{~m}, 3 \mathrm{H}), 7.27-7.22$ $(\mathrm{m}, 2 \mathrm{H}), 7.21-7.15(\mathrm{~m}, 3 \mathrm{H}), 6.37$ (s, 1H), 1.29 (s, 9H). ${ }^{13} \mathbf{C}$ NMR (100 MHz, $\left.\mathbf{C D C l}_{3}\right): \delta=168.96,157.74,146.26,140.44,138.80$, 137.76, 134.66, 134.48, 131.68, 131.65, 130.73, 130.49, 129.84, 129.06, 128.90, 128.47, $128.45,128.14,127.37,127.22,127.06,126.51,125.84,125.63,123.95,118.00,82.11$, 75.80, 27.83. HRMS (ESI): $[\mathrm{M}+\mathrm{H}]^{+}$calculated for $\mathrm{C}_{33} \mathrm{H}_{29}{ }^{35} \mathrm{ClNO}_{3}{ }^{+}$: 522.1830, found: 522.1831 . 
tert-butyl 2-((3,4-diphenylisoquinolin-1-yl)oxy)-2-(m-tolyl)acetate (4aha)

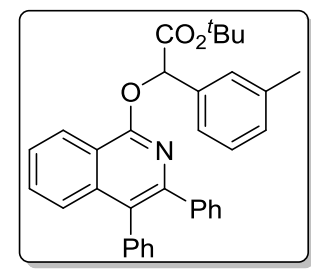

White solid; $33.1 \mathrm{mg}, 66 \%$ yield; ${ }^{1} \mathbf{H}$ NMR (400 MHz, $\mathbf{C D C l}_{3}$ ): $\delta=$ $8.51-8.42$ (m, 1H), $7.61-7.48$ (m, 5H), 7.46 - 7.39 (m, 2H), 7.39 $7.30(\mathrm{~m}, 4 \mathrm{H}), 7.29-7.21(\mathrm{~m}, 3 \mathrm{H}), 7.20-7.09(\mathrm{~m}, 3 \mathrm{H}), 6.36(\mathrm{~s}, 1 \mathrm{H})$, $2.44(\mathrm{~s}, 3 \mathrm{H}), 1.30(\mathrm{~s}, 9 \mathrm{H}) .{ }^{13} \mathbf{C}$ NMR (100 MHz, CDCl $\left.\mathbf{3}\right): \delta=169.45$, $158.05,146.29$, 140.55, 138.78, 138.32, 137.91, 135.78, 131.73, 131.69, 130.63, 130.55, 129.49 , 128.53, 128.48, 128.45, 127.34, 127.16, 127.00, 126.38, 125.61, 125.53, 124.91, 124.16, 118.13, 81.73, 76.60, 27.87, 21.61. HRMS (ESI): $[\mathrm{M}+\mathrm{H}]^{+}$calculated for $\mathrm{C}_{34} \mathrm{H}_{32} \mathrm{NO}_{3}{ }^{+}:$502.2377, found: 502.2376.

tert-butyl 2-((3,4-diphenylisoquinolin-1-yl)oxy)-2-(3-fluorophenyl)acetate (4aia)

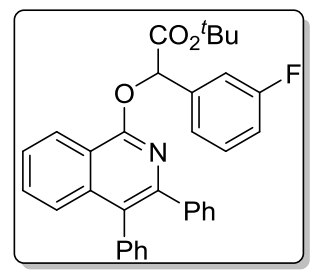

White solid; $39.4 \mathrm{mg}, 78 \%$ yield; ${ }^{\mathbf{H}} \mathbf{H}$ NMR (400 $\mathbf{M H z}, \mathbf{C D C l}_{3}$ ): $\delta=$ $8.49-8.42(\mathrm{~m}, 1 \mathrm{H}), 7.59-7.48(\mathrm{~m}, 5 \mathrm{H}), 7.45-7.32(\mathrm{~m}, 6 \mathrm{H}), 7.29-$ $7.21(\mathrm{~m}, 2 \mathrm{H}), 7.20-7.15(\mathrm{~m}, 3 \mathrm{H}), 7.15-7.05(\mathrm{~m}, 1 \mathrm{H}), 6.39(\mathrm{~s}, 1 \mathrm{H})$, 1.30 (s, 9H). ${ }^{19}$ F NMR (376 MHz, $\left.\mathbf{C D C l}_{3}\right): \delta=-112.36 .{ }^{13} \mathbf{C}$ NMR (100 MHz, $\left.\mathbf{C D C l}_{3}\right): \delta=168.83,162.95$ (d, $\left.J=246.1 \mathrm{~Hz}\right), 157.70,146.27,140.44$, $138.83,138.31(\mathrm{~d}, J=7.6 \mathrm{~Hz}), 137.78,131.70,131.65,130.75,130.50,130.18$ (d, $J=8.1$ $\mathrm{Hz}$ ), 128.46, 127.38, 127.23, 127.07, 126.55, 125.88, 125.63, 123.96, 123.28 (d, $J=2.8$ $\mathrm{Hz}), 118.02,115.66(\mathrm{~d}, J=21.1 \mathrm{~Hz}), 114.63(\mathrm{~d}, J=22.7 \mathrm{~Hz}), 82.18,75.85(\mathrm{~d}, J=1.9$ $\mathrm{Hz}$ ), 27.83. HRMS (ESI): $[\mathrm{M}+\mathrm{H}]^{+}$calculated for $\mathrm{C}_{33} \mathrm{H}_{29} \mathrm{FNO}_{3}{ }^{+}$: 506.2126, found: 506.2124 .

tert-butyl 2-(3-chlorophenyl)-2-((3,4-diphenylisoquinolin-1-yl)oxy)acetate (4aja)

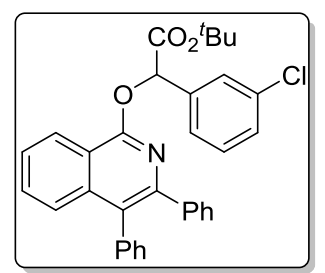

White solid; $30.7 \mathrm{mg}, 59 \%$ yield; ${ }^{1} \mathbf{H}$ NMR (400 $\mathbf{M H z}, \mathbf{C D C l}_{3}$ ): $\delta=$ $8.49-8.39(\mathrm{~m}, 1 \mathrm{H}), 7.80-7.76(\mathrm{~m}, 1 \mathrm{H}), 7.68-7.60(\mathrm{~m}, 1 \mathrm{H}), 7.59-$ $7.48(\mathrm{~m}, 3 \mathrm{H}), 7.42-7.31(\mathrm{~m}, 7 \mathrm{H}), 7.29-7.21(\mathrm{~m}, 2 \mathrm{H}), 7.20-7.11$ $(\mathrm{m}, 3 \mathrm{H}), 6.37$ (s, 1H), 1.30 (s, 9H). ${ }^{13} \mathbf{C}$ NMR (100 MHz, $\left.\mathbf{C D C l}_{3}\right): \delta$ $=168.76,157.67,146.26,140.43,138.82,137.88,137.76,134.56,131.70,131.64$, $130.76,130.50,129.93,129.09,128.89,128.46,127.82,127.38,127.23,127.07,126.56$, 125.89, 125.80, 125.63, 123.98, 118.00, 82.24, 75.81, 27.84. HRMS (ESI): $[\mathrm{M}+\mathrm{H}]^{+}$ calculated for $\mathrm{C}_{33} \mathrm{H}_{29}{ }^{35} \mathrm{ClNO}_{3}{ }^{+}$: 522.1830, found: 522.1835 . 
tert-butyl 2-((3,4-diphenylisoquinolin-1-yl)oxy)-2-(naphthalen-2-yl)acetate (4aka)

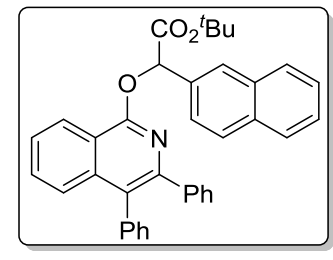

White solid; $10.1 \mathrm{mg}, 18 \%$ yield; ${ }^{1} \mathbf{H}$ NMR (400 $\left.\mathbf{M H z}, \mathbf{C D C l}_{3}\right): \delta=$ $9.06(\mathrm{~s}, 1 \mathrm{H}), 8.10-8.06(\mathrm{~m}, 2 \mathrm{H}), 7.84-7.80(\mathrm{~m}, 3 \mathrm{H}), 7.53-7.40$ $(\mathrm{m}, 10 \mathrm{H}), 7.36-7.33(\mathrm{~m}, 3 \mathrm{H}), 7.22-7.15(\mathrm{~m}, 3 \mathrm{H}), 6.48(\mathrm{~s}, 1 \mathrm{H})$, 1.31 (s, 9H). ${ }^{13} \mathbf{C}$ NMR (100 MHz, $\left.\mathbf{C D C l}_{3}\right): \delta=169.30,158.51$, 144.22 , 140.60, 138.19, 135.91, 135.46, 134.53, 131.88, 131.84, 131.61, 130.63, 129.09, $128.84,128.76,128.63,128.58,128.47,127.93$, 127.37, 127.33, 127.28, 126.96, 125.99, 125.00, 124.53, 124.29, 117.44, 81.91, 76.70, 27.89. HRMS (ESI): $[\mathrm{M}+\mathrm{H}]^{+}$calculated for $\mathrm{C}_{37} \mathrm{H}_{32} \mathrm{NO}_{3}{ }^{+}:$538.2377, found: 538.2376.

methyl 2-((3,4-diphenylisoquinolin-1-yl)oxy)-2-phenylacetate (4ala)

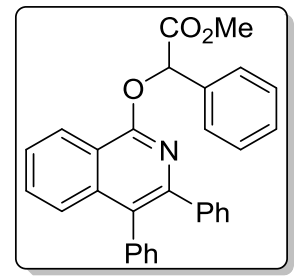

White solid; $28.0 \mathrm{mg}, 63 \%$ yield; ${ }^{1} \mathbf{H}$ NMR (400 $\mathbf{M H z}, \mathbf{C D C l}_{3}$ ): $\delta=$ $8.50-8.42(\mathrm{~m}, 1 \mathrm{H}), 7.80-7.72(\mathrm{~m}, 2 \mathrm{H}), 7.58-7.51(\mathrm{~m}, 3 \mathrm{H}), 7.50-$ $7.38(\mathrm{~m}, 4 \mathrm{H}), 7.37-7.26(\mathrm{~m}, 5 \mathrm{H}), 7.20-7.09(\mathrm{~m}, 4 \mathrm{H}), 6.44(\mathrm{~s}, 1 \mathrm{H})$, 3.72 (s, 3H). ${ }^{13} \mathbf{C}$ NMR (100 MHz, $\left.\mathbf{C D C l}_{3}\right): \delta=170.90,157.81$, 146.16, 140.32, 138.81, 137.73, 135.28, 131.71, 131.56, 130.76, 130.34, 129.07, 128.83, 128.63, 128.31, 127.83, 127.41, 127.23, 127.12, 126.51, 125.88, 125.62, 124.04, 117.99, 76.26, 52.27. HRMS (ESI): $[\mathrm{M}+\mathrm{H}]^{+}$calculated for $\mathrm{C}_{30} \mathrm{H}_{24} \mathrm{NO}_{3}{ }^{+}:$446.1751, found: 446.1750 .

ethyl 2-((3,4-diphenylisoquinolin-1-yl)oxy)-2-phenylacetate (4ama)

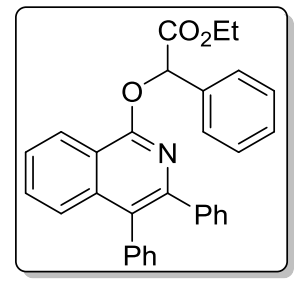

White solid; $32.6 \mathrm{mg}, 71 \%$ yield; ${ }^{1} \mathbf{H}$ NMR (400 MHz, $\mathbf{C D C l}_{3}$ ): $\delta=$ $8.48-8.44(\mathrm{~m}, 1 \mathrm{H}), 7.78-7.75(\mathrm{~m}, 2 \mathrm{H}), 7.56-7.49(\mathrm{~m}, 3 \mathrm{H}), 7.47-$ $7.43(\mathrm{~m}, 2 \mathrm{H}), 7.42-7.27(\mathrm{~m}, 7 \mathrm{H}), 7.19-7.14(\mathrm{~m}, 4 \mathrm{H}), 6.44(\mathrm{~s}, 1 \mathrm{H})$, $4.27-4.09(\mathrm{~m}, 2 \mathrm{H}), 1.14(\mathrm{t}, \mathrm{J}=7.1 \mathrm{~Hz}, 3 \mathrm{H}) .{ }^{13} \mathrm{C}$ NMR (100 MHz, $\left.\mathbf{C D C l}_{3}\right): \delta=170.39,157.87,146.19,140.37,138.78,137.76,135.41,131.69,131.60$, 130.72 , 130.38, 128.97, 128.78, 128.58, 128.33, 127.81, 127.38, 127.20, 127.07, 126.47, 125.81, 125.58, 124.06, 118.01, 76.30, 61.27, 14.04. HRMS (ESI): $[\mathrm{M}+\mathrm{H}]^{+}$calculated for $\mathrm{C}_{31} \mathrm{H}_{26} \mathrm{NO}_{3}{ }^{+}$: 460.1907, found: 460.1906 . 
benzyl 2-((3,4-diphenylisoquinolin-1-yl)oxy)-2-phenylacetate (4ana)

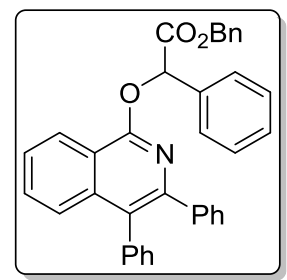

White solid; $36.5 \mathrm{mg}, 70 \%$ yield; ${ }^{1} \mathbf{H}$ NMR (400 MHz, $\mathbf{C D C l}_{3}$ ): $\delta=$ $8.51-8.38(\mathrm{~m}, 1 \mathrm{H}), 7.81-7.68(\mathrm{~m}, 2 \mathrm{H}), 7.55-7.47(\mathrm{~m}, 3 \mathrm{H}), 7.45-$ $7.36(\mathrm{~m}, 3 \mathrm{H}), 7.36-7.22(\mathrm{~m}, 6 \mathrm{H}), 7.21-7.13(\mathrm{~m}, 4 \mathrm{H}), 7.12-7.01(\mathrm{~m}$, $5 \mathrm{H}), 6.53(\mathrm{~s}, 1 \mathrm{H}), 5.11(\mathrm{~s}, 2 \mathrm{H}) .{ }^{13} \mathbf{C} \mathbf{N M R}\left(\mathbf{1 0 0} \mathbf{M H z}, \mathbf{C D C l}_{3}\right): \delta=$ 170.28, 157.83, 146.24, 140.29, 138.78, 137.74, 135.49, 135.19, 131.67, 131.60, 130.76, $130.35,129.04,128.81,128.57,128.43,128.32$, 128.10, 127.90, 127.83, 127.45, 127.20, $127.05,126.51,125.90,125.59,124.03,118.01,76.15,66.91$. HRMS (ESI): $[\mathrm{M}+\mathrm{H}]^{+}$ calculated for $\mathrm{C}_{36} \mathrm{H}_{28} \mathrm{NO}_{3}^{+}$: 522.2064, found: 522.2070.

tert-butyl 2-((3,4-di-p-tolylisoquinolin-1-yl)oxy)-2-phenylacetate (4aab)

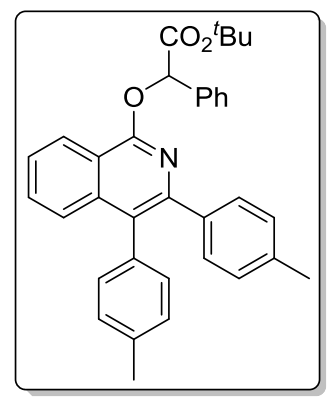

White solid; $33.5 \mathrm{mg}, 65 \%$ yield; ${ }^{1} \mathbf{H}$ NMR (400 $\mathbf{M H z}, \mathbf{C D C l}_{3}$ ): $\delta=$ $8.45-8.43(\mathrm{~m}, 1 \mathrm{H}), 7.76-7.74(\mathrm{~m}, 2 \mathrm{H}), 7.55-7.34(\mathrm{~m}, 8 \mathrm{H}), 7.19$ - $7.14(\mathrm{~m}, 3 \mathrm{H}), 7.11-7.10(\mathrm{~m}, 1 \mathrm{H}), 7.00-6.98(\mathrm{~m}, 2 \mathrm{H}), 6.41(\mathrm{~s}$, 1H), 2.38 (s, 3H), 2.28 (s, 3H), 1.32 (s, 9H). ${ }^{13}$ C NMR (100 MHz, $\left.\mathbf{C D C l}_{3}\right): \delta=169.38,157.76,146.13,139.05,137.73,136.64,136.62$, $136.00,134.95,131.45,130.47,130.42$, 129.28, 129.14, 128.62, 128.12 , 127.72, 126.16, 125.56, 125.28, 124.02, 117.99, 81.74, 76.40, 27.88, 21.38, 21.25. HRMS (ESI): $[\mathrm{M}+\mathrm{H}]^{+}$calculated for $\mathrm{C}_{35} \mathrm{H}_{34} \mathrm{NO}_{3}{ }^{+}: 516.2533$, found: 516.2532 .

tert-butyl 2-((3,4-bis(4-methoxyphenyl)isoquinolin-1-yl)oxy)-2-phenylacetate (4aac)

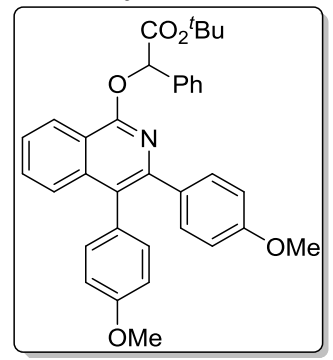

White solid; $38.8 \mathrm{mg}, 71 \%$ yield; ${ }^{1} \mathbf{H}$ NMR (400 $\left.\mathbf{M H z}, \mathbf{C D C l}_{3}\right): \delta=$ $8.48-8.37(\mathrm{~m}, 1 \mathrm{H}), 7.83-7.71(\mathrm{~m}, 2 \mathrm{H}), 7.61-7.53(\mathrm{~m}, 2 \mathrm{H}), 7.50$ - $7.33(\mathrm{~m}, 6 \mathrm{H}), 7.21-7.08(\mathrm{~m}, 2 \mathrm{H}), 7.00-6.86(\mathrm{~m}, 2 \mathrm{H}), 6.79-$ $6.62(\mathrm{~m}, 2 \mathrm{H}), 6.38(\mathrm{~s}, 1 \mathrm{H}), 3.85(\mathrm{~s}, 3 \mathrm{H}), 3.77(\mathrm{~s}, 3 \mathrm{H}), 1.31(\mathrm{~s}, 9 \mathrm{H})$. ${ }^{13}$ C NMR (100 MHz, $\left.\mathbf{C D C l}_{3}\right): \delta=169.41,158.71,158.63,157.71$, $145.91,139.24,135.96,133.14,132.67,132.65,131.77,130.52,130.27,128.68,128.64$, $127.71,126.06,125.45,124.54,124.06,117.90,114.07,114.05,112.85,81.78,76.48$, 55.32, 55.24, 27.89. HRMS (ESI): $[\mathrm{M}+\mathrm{H}]^{+}$calculated for $\mathrm{C}_{35} \mathrm{H}_{34} \mathrm{NO}_{5}{ }^{+}$: 548.2430, found: 548.2434 . 
tert-butyl 2-((3,4-bis(4-fluorophenyl)isoquinolin-1-yl)oxy)-2-phenylacetate (4aad)

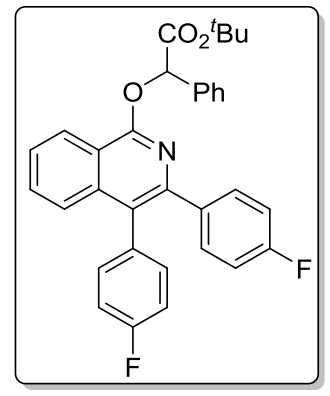

White solid; $38.7 \mathrm{mg}, 74 \%$ yield; ${ }^{1} \mathbf{H}$ NMR (400 $\mathbf{M H z}, \mathbf{C D C l}_{3}$ ): $\delta=$ $8.48-8.43(\mathrm{~m}, 1 \mathrm{H}), 7.78-7.71(\mathrm{~m}, 2 \mathrm{H}), 7.64-7.52(\mathrm{~m}, 2 \mathrm{H}), 7.51$

- $7.47(\mathrm{~m}, 1 \mathrm{H}), 7.46-7.43(\mathrm{~m}, 2 \mathrm{H}), 7.43$ - 7.40 (m, 1H), 7.39 $7.33(\mathrm{~m}, 2 \mathrm{H}), 7.26-7.13(\mathrm{~m}, 2 \mathrm{H}), 7.13-7.02(\mathrm{~m}, 2 \mathrm{H}), 6.95-6.84$ $(\mathrm{m}, 2 \mathrm{H}), 6.34(\mathrm{~s}, 1 \mathrm{H}), 1.29$ (s, 9H). ${ }^{19}$ F NMR (376 MHz, $\left.\mathbf{C D C l}_{3}\right): \delta$ $=-114.58,-114.82 .{ }^{13} \mathbf{C}$ NMR (100 MHz, $\left.\mathbf{C D C l}_{3}\right): \delta=169.21$, $162.17(\mathrm{~d}, J=246.8 \mathrm{~Hz}), 162.02(\mathrm{~d}, J=247.2 \mathrm{~Hz}), 158.14,145.57,138.70,136.41$ (d, $J$ $=3.2 \mathrm{~Hz}), 135.70,133.56(\mathrm{~d}, J=3.4 \mathrm{~Hz}), 133.23(\mathrm{~d}, J=7.1 \mathrm{~Hz}), 133.16(\mathrm{~d}, J=7.0 \mathrm{~Hz})$, $132.17(\mathrm{~d}, J=8.0 \mathrm{~Hz}), 130.92,128.79,128.69,127.69,126.62,125.22,124.45,124.23$, 118.13, $115.67(\mathrm{~d}, J=21.3 \mathrm{~Hz}), 114.41(\mathrm{~d}, J=21.3 \mathrm{~Hz}), 81.86,76.68,27.85$. HRMS (ESI): $[\mathrm{M}+\mathrm{H}]^{+}$calculated for $\mathrm{C}_{33} \mathrm{H}_{28} \mathrm{~F}_{2} \mathrm{NO}_{3}{ }^{+}:$524.2032, found: 524.2031.

tert-butyl 2-((3,4-bis(4-chlorophenyl)isoquinolin-1-yl)oxy)-2-phenylacetate (4aae)

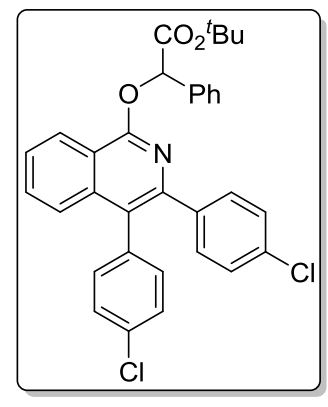

White solid; $40.5 \mathrm{mg}, 73 \%$ yield; ${ }^{1} \mathbf{H}$ NMR (400 $\mathbf{M H z}, \mathbf{C D C l}_{3}$ ): $\delta=$ $8.54-8.34(\mathrm{~m}, 1 \mathrm{H}), 7.80-7.68(\mathrm{~m}, 2 \mathrm{H}), 7.61-7.49$ (m, 3H), 7.48 - $7.39(\mathrm{~m}, 3 \mathrm{H}), 7.38-7.30(\mathrm{~m}, 4 \mathrm{H}), 7.20-7.12(\mathrm{~m}, 4 \mathrm{H}), 6.33(\mathrm{~s}$, 1H), 1.30 (s, 9H). ${ }^{13} \mathbf{C}$ NMR (100 MHz, $\left.\mathbf{C D C l}_{3}\right): \delta=169.15,158.28$, $145.24,138.70,138.44,136.05,135.63,133.47,133.27,132.94$, $132.91,131.77,131.03,128.97,128.93,128.82,128.70,127.76$, 127.69, 126.81, 125.19, 124.50, 124.28, 118.22, 81.93, 76.67, 27.87. HRMS (ESI): $[\mathrm{M}+\mathrm{H}]^{+}$calculated for $\mathrm{C}_{33} \mathrm{H}_{28}{ }^{35} \mathrm{Cl}_{2} \mathrm{NO}_{3}{ }^{+}:$556.1441, found: 556.1440.

tert-butyl 2-((3,4-bis(4-bromophenyl)isoquinolin-1-yl)oxy)-2-phenylacetate (4aaf)

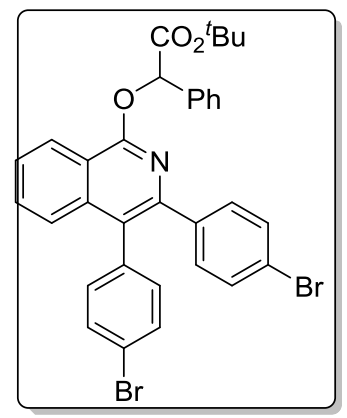

White solid; $41.2 \mathrm{mg}, 64 \%$ yield; ${ }^{1} \mathbf{H}$ NMR (400 $\mathbf{M H z}, \mathbf{C D C l}_{3}$ ): $\delta=$ $8.53-8.31(\mathrm{~m}, 1 \mathrm{H}), 7.81-7.67(\mathrm{~m}, 2 \mathrm{H}), 7.61-7.52(\mathrm{~m}, 3 \mathrm{H}), 7.51$ - $7.47(\mathrm{~m}, 2 \mathrm{H}), 7.47-7.37(\mathrm{~m}, 3 \mathrm{H}), 7.37$ - $7.30(\mathrm{~m}, 2 \mathrm{H}), 7.29$ $7.20(\mathrm{~m}, 2 \mathrm{H}), 7.15-7.04(\mathrm{~m}, 2 \mathrm{H}), 6.33(\mathrm{~s}, 1 \mathrm{H}), 1.30(\mathrm{~s}, 9 \mathrm{H}) .{ }^{13} \mathrm{C}$ NMR (100 MHz, $\left.\mathbf{C D C l}_{3}\right): \delta=169.12,158.29,145.18,139.12$, $138.35,136.50,135.60,133.25,133.23,132.08,131.93,131.88$, $131.05,130.72$, 128.81, 128.70, 127.68, 126.84, 125.17, 124.48, 124.27, 121.65, 118.20, 81.93, 76.66, 27.87. HRMS (ESI): $[\mathrm{M}+\mathrm{H}]^{+}$calculated for $\mathrm{C}_{33} \mathrm{H}_{28}{ }^{79} \mathrm{Br}_{2} \mathrm{NO}_{3}{ }^{+}:$644.0430, 
found: 644.0428 .

tert-butyl 2-((3,4-bis(4-(tert-butyl)phenyl)isoquinolin-1-yl)oxy)-2-phenylacetate (4aag)

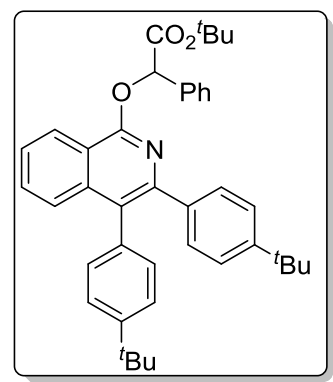

White solid; $46.1 \mathrm{mg}, 77 \%$ yield; ${ }^{1} \mathbf{H}$ NMR (400 $\left.\mathbf{M H z}, \mathbf{C D C l}_{3}\right): \delta=$ $8.56-8.40(\mathrm{~m}, 1 \mathrm{H}), 7.81-7.73(\mathrm{~m}, 2 \mathrm{H}), 7.65-7.57(\mathrm{~m}, 1 \mathrm{H}), 7.56$ - $7.51(\mathrm{~m}, 1 \mathrm{H}), 7.51-7.42(\mathrm{~m}, 3 \mathrm{H}), 7.41-7.34(\mathrm{~m}, 5 \mathrm{H}), 7.24-$ $7.10(\mathrm{~m}, 4 \mathrm{H}), 6.41(\mathrm{~s}, 1 \mathrm{H}), 1.37(\mathrm{~s}, 9 \mathrm{H}), 1.31-1.26(\mathrm{~m}, 18 \mathrm{H}) .{ }^{13} \mathrm{C}$ NMR (100 MHz, $\left.\mathbf{C D C l}_{\mathbf{3}}\right): \delta=169.44,157.71,150.05,149.74$, $146.09,139.06,137.65,135.99,134.90,131.26,130.45,130.18$, $128.64,127.71,126.16,125.70,125.39,125.32$, 125.27, 124.20, 123.99, 117.96, 81.73, 76.49, 34.65, 34.48, 31.52, 31.36, 27.84. HRMS (ESI): $[\mathrm{M}+\mathrm{H}]^{+}$calculated for $\mathrm{C}_{41} \mathrm{H}_{46} \mathrm{NO}_{3}^{+}$: 600.3472, found: 600.3478 .

tert-butyl 2-((3,4-bis(4-(trifluoromethyl)phenyl)isoquinolin-1-yl)oxy)-2-phenylacetate (4aah)

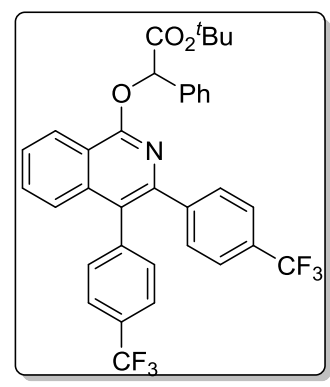

White solid; $41.7 \mathrm{mg}, 67 \%$ yield; ${ }^{1} \mathbf{H}$ NMR (400 $\mathbf{M H z}, \mathbf{C D C l}_{3}$ ): $\delta=$ $8.62-8.38(\mathrm{~m}, 1 \mathrm{H}), 7.78-7.71(\mathrm{~m}, 2 \mathrm{H}), 7.70-7.64(\mathrm{~m}, 2 \mathrm{H}), 7.63$ - $7.56(\mathrm{~m}, 2 \mathrm{H}), 7.52-7.45(\mathrm{~m}, 7 \mathrm{H}), 7.44-7.31(\mathrm{~m}, 3 \mathrm{H}), 6.34(\mathrm{~s}$, 1H), 1.29 (s, 9H). ${ }^{19} \mathbf{F}$ NMR (376 $\left.\mathbf{M H z}, \mathbf{C D C l}_{3}\right): \delta=-62.31,-$ 62.41. ${ }^{13} \mathbf{C}$ NMR (100 MHz, $\left.\mathbf{C D C l}_{3}\right): \delta=169.07,158.63,145.10$, $143.68,141.33,138.16,135.52,132.05,132.03,131.33,130.72$, 129.85 (q, $J=32.6 \mathrm{~Hz}), 129.31$ (q, $J=32.3 \mathrm{~Hz}), 128.91,128.76,127.71,127.28,125.66$ $(\mathrm{q}, J=3.3 \mathrm{~Hz}), 125.16,124.94,124.55(\mathrm{q}, J=3.1 \mathrm{~Hz}), 124.41,124.23$ (q, $J=273.5 \mathrm{~Hz})$, $124.22(\mathrm{q}, J=272.2 \mathrm{~Hz}), 118.43,82.04,76.84,27.83$. HRMS (ESI): $[\mathrm{M}+\mathrm{H}]^{+}$calculated for $\mathrm{C}_{35} \mathrm{H}_{28} \mathrm{~F}_{6} \mathrm{NO}_{3}{ }^{+}$: 624.1968, found: 624.1962.

tert-butyl 2-((3,4-di-m-tolylisoquinolin-1-yl)oxy)-2-phenylacetate (4aai)

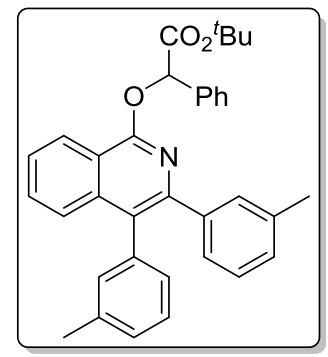

White solid; $39.1 \mathrm{mg}, 76 \%$ yield; ${ }^{1} \mathbf{H}$ NMR (400 $\left.\mathbf{M H z}, \mathbf{C D C l}_{3}\right): \delta=$ $8.45-8.42(\mathrm{~m}, 1 \mathrm{H}), 7.75-7.73(\mathrm{~m}, 2 \mathrm{H}), 7.55-7.41(\mathrm{~m}, 5 \mathrm{H})$, $7.39-7.33(\mathrm{~m}, 3 \mathrm{H}), 7.18-7.13(\mathrm{~m}, 3 \mathrm{H}), 7.11-7.09(\mathrm{~m}, 1 \mathrm{H})$, $6.99-6.97(\mathrm{~m}, 2 \mathrm{H}), 6.40(\mathrm{~s}, 1 \mathrm{H}), 2.38(\mathrm{~s}, 3 \mathrm{H}), 2.28(\mathrm{~s}, 3 \mathrm{H}), 1.31(\mathrm{~s}$, 
9H). ${ }^{13}$ C NMR (100 MHz, $\left.\mathbf{C D C l}_{3}\right): \delta=169.39,157.76,146.13,139.05,137.74,136.65$, 136.62, 136.00, 134.95, 131.48, 131.45, 130.48, 130.42, 129.29, 129.14, 128.66, 128.63, $128.12,127.72,126.17,125.57,125.29,124.02,117.99,81.74,76.40,27.88,21.37,21.24$. HRMS (ESI): $[\mathrm{M}+\mathrm{H}]^{+}$calculated for $\mathrm{C}_{35} \mathrm{H}_{34} \mathrm{NO}_{3}{ }^{+}: 516.2533$, found: 516.2533 .

tert-butyl 2-((3,4-di-o-tolylisoquinolin-1-yl)oxy)-2-phenylacetate (4aaj)

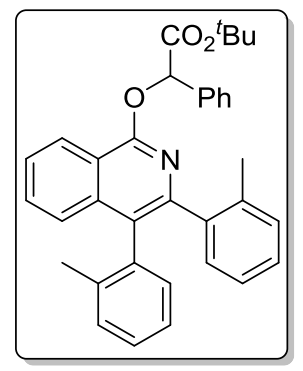

White solid; $41.2 \mathrm{mg}, 80 \%$ yield; ${ }^{1} \mathbf{H}$ NMR (400 $\left.\mathbf{M H z}, \mathbf{C D C l}_{3}\right): \delta=$ $8.45-8.43(\mathrm{~m}, 1 \mathrm{H}), 7.76-7.74(\mathrm{~m}, 2 \mathrm{H}), 7.56-7.47(\mathrm{~m}, 3 \mathrm{H}), 7.46-$ $7.42(\mathrm{~m}, 2 \mathrm{H}), 7.39-7.34(\mathrm{~m}, 3 \mathrm{H}), 7.18-7.00(\mathrm{~m}, 4 \mathrm{H}), 6.99(\mathrm{~d}, J=$ $8.0 \mathrm{~Hz}, 2 \mathrm{H}), 6.41$ (s, 1H), 2.38 (s, 3H), 2.28 (s, 3H), 1.32 (s, 9H). ${ }^{13} \mathbf{C}$ NMR (100 MHz, $\left.\mathbf{C D C l}_{3}\right): \delta=169.38,157.75,146.13,139.04$, $137.73,136.62,136.60,135.98,134.94,131.45,130.47,130.43$, $130.40,129.28,129.14,128.62,128.12,128.08,127.71,126.16,125.55,125.28,124.00$, 117.98, 81.72, 76.40, 27.87, 21.35, 21.22. HRMS (ESI): $[\mathrm{M}+\mathrm{H}]^{+}$calculated for $\mathrm{C}_{35} \mathrm{H}_{34} \mathrm{NO}_{3}^{+}$: 516.2533, found: 516.2530.

tert-butyl 2-((3,4-bis(2-chlorophenyl)isoquinolin-1-yl)oxy)-2-phenylacetate (4aak)

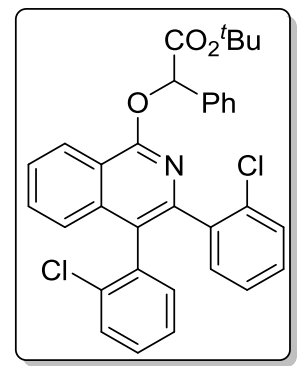

White solid; $24.9 \mathrm{mg}, 45 \%$ yield; ${ }^{1} \mathbf{H}$ NMR (400 $\left.\mathbf{M H z}, \mathbf{C D C l}_{3}\right): \delta=$ $8.52-8.50(\mathrm{~m}, 1 \mathrm{H}), 7.72-7.70(\mathrm{~m}, 2 \mathrm{H}), 7.62-7.56(\mathrm{~m}, 2 \mathrm{H}), 7.45-$ $7.27(\mathrm{~m}, 7 \mathrm{H}), 7.23-7.12(\mathrm{~m}, 4 \mathrm{H}), 7.07-7.03(\mathrm{~m}, 1 \mathrm{H}), 6.39(\mathrm{~s}, 1 \mathrm{H})$, 1.28 (s, 9H). ${ }^{13} \mathbf{C}$ NMR (100 $\left.\mathbf{~ M H z}, \mathbf{C D C l}_{3}\right): \delta=169.11,158.38$, $144.92,142.13,139.64,138.25,135.62,134.28,133.32,131.15$, $130.63,130.37,130.33,130.17,128.99,128.88,128.80,128.69$, $127.71,126.99,125.23,124.58,124.29,122.55,121.91,118.29,82.05,76.57,27.90$. HRMS (ESI): $[\mathrm{M}+\mathrm{H}]^{+}$calculated for $\mathrm{C}_{33} \mathrm{H}_{28}{ }^{35} \mathrm{Cl}_{2} \mathrm{NO}_{3}{ }^{+}: 556.1441$, found: 556.1440 .

tert-butyl 2-((4-([1,1'-biphenyl]-4-yl)-3-phenylisoquinolin-1-yl)oxy)-2-phenylacetate (4aal+4aal')

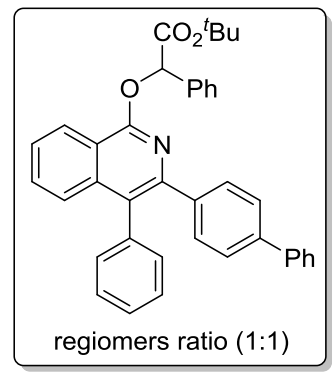

White solid; $32.7 \mathrm{mg}, 58 \%$ yield; ${ }^{1} \mathbf{H}$ NMR (400 $\left.\mathbf{M H z}, \mathbf{C D C l}_{3}\right): \delta=$ $8.54-8.21(\mathrm{~m}, 1 \mathrm{H}), 7.90-7.73(\mathrm{~m}, 2 \mathrm{H}), 7.67-7.61(\mathrm{~m}, 2 \mathrm{H}), 7.60$ $-7.55(\mathrm{~m}, 2 \mathrm{H}), 7.55-7.50(\mathrm{~m}, 2 \mathrm{H}), 7.49-7.44(\mathrm{~m}, 4 \mathrm{H}), 7.44-$ $7.34(\mathrm{~m}, 5 \mathrm{H}), 7.34-7.16(\mathrm{~m}, 3 \mathrm{H}), 7.11-7.01(\mathrm{~m}, 1 \mathrm{H}), 6.96-6.76$ 
(m, 1H), $6.41(\mathrm{~s}, 1 \mathrm{H}), 6.36(\mathrm{~s}, 1 \mathrm{H}), 1.33-1.26(\mathrm{~m}, 9 \mathrm{H}) .{ }^{13} \mathbf{C}$ NMR (100 MHz, $\left.\mathbf{C D C l}_{3}\right): \delta$ $=169.28,169.24,163.38,163.22,160.93,160.76,158.11,158.03,146.08,145.27$, $140.73,140.54,139.91,139.74,139.31,138.76,138.71,136.68,136.54,136.51,135.78$, $135.73,133.73,133.70,133.35,133.28,133.21,132.27,132.19,132.06,132.00,130.92$, $130.82,128.89,128.77,128.67,127.70,127.68,127.48,127.33$, 127.16, 127.03, 126.54, $126.14,125.48,125.25,125.15,124.57,124.19,124.13,118.13,118.11,115.77,115.69$, 115.56, 115.48, 114.44, 114.23, 81.83, 81.80, 76.67, 76.59, 27.86, 27.83. HRMS (ESI): $[\mathrm{M}+\mathrm{H}]^{+}$calculated for $\mathrm{C}_{39} \mathrm{H}_{34} \mathrm{NO}_{3}{ }^{+}: 564.2533$, found: 564.2530 .

tert-butyl 2-((3-methyl-4-phenylisoquinolin-1-yl)oxy)-2-phenylacetate (4aam)

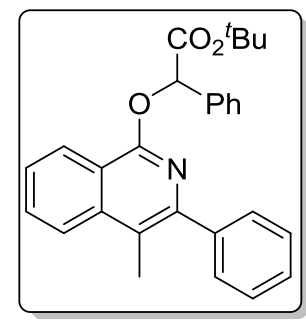

White solid; $31.5 \mathrm{mg}, 74 \%$ yield; ${ }^{1} \mathbf{H}$ NMR (400 $\left.\mathbf{M H z}, \mathbf{C D C l}_{3}\right): \delta=$ $8.43(\mathrm{~d}, J=8.2 \mathrm{~Hz}, 1 \mathrm{H}), 7.94-7.92(\mathrm{~m}, 1 \mathrm{H}), 7.72-7.68(\mathrm{~m}, 3 \mathrm{H})$, $7.64-7.61(\mathrm{~m}, 2 \mathrm{H}), 7.56-7.52(\mathrm{~m}, 1 \mathrm{H}), 7.47-7.40(\mathrm{~m}, 4 \mathrm{H}), 7.39-$ 7.35 (m, 2H), 6.29 (s, 1H), 2.57 (s, 3H), 1.26 (s, 9H). ${ }^{13} \mathbf{C}$ NMR (100 MHz, $\left.\mathbf{C D C l}_{3}\right): \delta=169.34,156.91,147.28,141.19,138.85,136.04$, 130.61, 130.30, 128.58, 127.76, 127.68, 127.39, 126.14, 124.57, 123.60, 118.29, 118.27, 81.56, 77.42, 77.10, 76.78, 76.27, 27.79, 15.25. HRMS (ESI): $[\mathrm{M}+\mathrm{H}]^{+}$calculated for $\mathrm{C}_{28} \mathrm{H}_{28} \mathrm{NO}_{3}^{+}$: 426.2064, found: 426.2064 .

tert-butyl 2-((3,4-dimethylisoquinolin-1-yl)oxy)-2-phenylacetate (4aan)

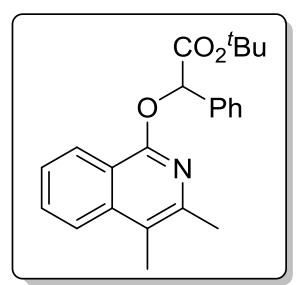

White solid; $26.5 \mathrm{mg}, 73 \%$ yield; ${ }^{1} \mathbf{H}$ NMR (400 $\left.\mathbf{M H z}, \mathbf{C D C l}_{3}\right): \delta=$ $8.35(\mathrm{~d}, J=8.3 \mathrm{~Hz}, 1 \mathrm{H}), 7.85-7.79(\mathrm{~m}, 1 \mathrm{H}), 7.75-7.69(\mathrm{~m}, 2 \mathrm{H})$, $7.67-7.59(\mathrm{~m}, 1 \mathrm{H}), 7.48-7.35(\mathrm{~m}, 4 \mathrm{H}), 6.20(\mathrm{~s}, 1 \mathrm{H}), 2.54(\mathrm{~s}, 3 \mathrm{H})$, 2.44 (s, 3H), 1.39 (s, 9H). ${ }^{13} \mathbf{C}$ NMR (100 $\left.\mathbf{~ M H z}, \mathbf{C D C l}_{3}\right): \delta=169.47$, 156.61, 145.08, 138.25, 136.05, 130.24, 128.58, 127.63, 125.06, 124.51, 122.62, 117.72, 81.46, 76.29, 27.98, 22.32, 13.50. HRMS (ESI): $[\mathrm{M}+\mathrm{H}]^{+}$calculated for $\mathrm{C}_{23} \mathrm{H}_{26} \mathrm{NO}_{3}{ }^{+}$: 364.1907, found: 364.1911 .

tert-butyl (Z)-2-((methoxyimino)(phenyl)methoxy)-2-phenylacetate (5)

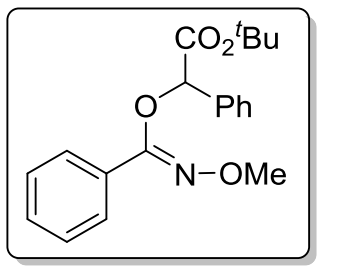

Colorless oil; $29.3 \mathrm{mg}, 86 \%$ yield; ${ }^{1} \mathbf{H}$ NMR (400 $\left.\mathbf{~ M H z}, \mathbf{C D C l}_{\mathbf{3}}\right): \delta$ $=7.84-7.75(\mathrm{~m}, 2 \mathrm{H}), 7.60-7.49(\mathrm{~m}, 2 \mathrm{H}), 7.43-7.37(\mathrm{~m}, 3 \mathrm{H})$, 
7.36 - 7.26 (m, 3H), 6.19 (s, 1H), 3.88 (s, 3H), 1.38 (s, 9H). ${ }^{13}$ C NMR (100 MHz, $\left.\mathbf{C D C l}_{3}\right): \delta=168.43,151.69,136.13,131.63,129.97,128.94,128.65,128.19,127.39$, 127.20, 82.22, 81.68, 62.43, 27.95. HRMS (ESI): $[\mathrm{M}+\mathrm{H}]^{+}$calculated for $\mathrm{C}_{20} \mathrm{H}_{24} \mathrm{NO}_{4}{ }^{+}$: 342.1700 , found: 342.1698 .

\section{4-methyl-3-phenylisoquinolin-1(2H)-one (8)}

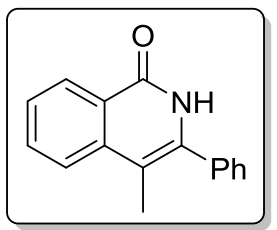

White solide; $21.0 \mathrm{mg}, 89 \%$ yield; ${ }^{1} \mathbf{H}$ NMR (400 $\left.\mathbf{M H z}, \mathbf{C D C l}_{\mathbf{3}}\right): \delta=$ $10.09(\mathrm{~s}, 1 \mathrm{H}), 8.39$ (d, J = $7.9 \mathrm{~Hz}, 1 \mathrm{H}), 7.73-7.71(\mathrm{~m}, 2 \mathrm{H}), 7.55-$ $7.42(\mathrm{~m}, 8 \mathrm{H}), 2.26(\mathrm{~s}, 4 \mathrm{H}) .{ }^{13} \mathbf{C}$ NMR (100 MHz, $\left.\mathbf{C D C l}_{\mathbf{3}}\right): \delta=162.87$, $138.88,137.00,135.26,132.66,129.48,129.01,128.62,127.71$, $126.29,125.37,123.63,109.14,13.91$.

methyl 2-hydroxy-2-phenylacetate (10)

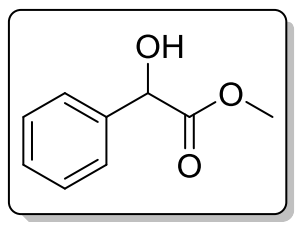

White solide; $27.8 \mathrm{mg}, 56 \%$ yield; ${ }^{1} \mathbf{H}$ NMR (400 $\left.\mathbf{M H z}, \mathbf{C D C l}_{3}\right): \delta=$ $7.43-7.40$ (m, 2H), $7.39-7.35(\mathrm{~m}, 2 \mathrm{H}), 7.35-7.30(\mathrm{~m}, 1 \mathrm{H}), 5.18$ (s, 1H), 3.75 (s, 3H). ${ }^{13} \mathbf{C}$ NMR (100 MHz, $\left.\mathbf{C D C l}_{\mathbf{3}}\right): \delta=174.20,138.23$, $128.68,128.58,126.65,72.91,53.14$.

\section{(D) X-Ray Crystallographic Data}

Single crystal X-ray diffraction data were collected on an Agilent Technologies SuperNova X-ray diffractometer system equipped with a $\mathrm{Cu}$ sealed tube $(\lambda=1.54184)$ at $50 \mathrm{kV}$ and $0.80 \mathrm{~mA}$. The structures were solved by direct methods with Olex $2^{[5]}$ program suite, and refined with the XL refinement package. ${ }^{[6]}$ The CCDC number for the compound 4baa is 1908915 . The supplementary crystallographic data for this paper can be obtained free of charge via www.ccdc.cam.ac.uk/conts/retrieving.html. 


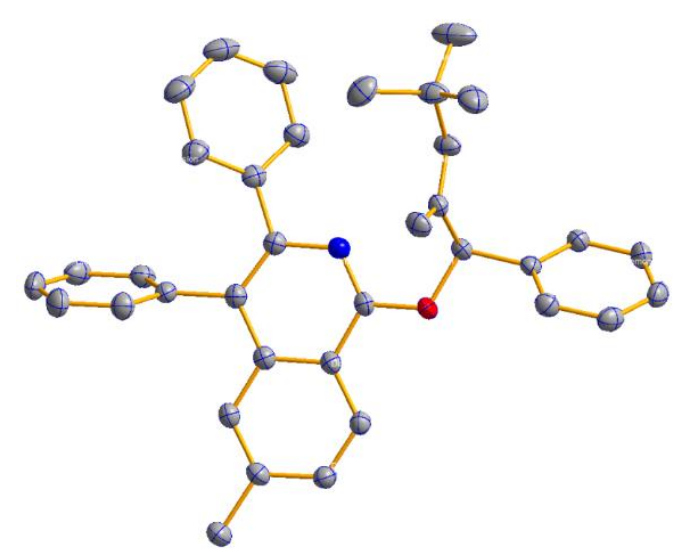

Figure S1. The structure of 4 baa with displacement ellipsoids drawn at $50 \%$ probability level.

Table S9. Crystal data and structure refinement for Compound 4baa

\begin{tabular}{|l|l|}
\hline Identification code $(\mathrm{CCDC})$ & $18090301(1908915)$ \\
\hline Empirical formula & $\mathrm{C}_{34} \mathrm{H}_{31} \mathrm{NO}_{3}$ \\
\hline Formula weight & 501.60 \\
\hline Temperature/K & $150.00(10)$ \\
\hline Crystal system & orthorhombic \\
\hline Space group & Pbca \\
\hline $\mathrm{a} / \AA$ & $15.54737(15)$ \\
\hline $\mathrm{b} / \AA$ & $16.85576(16)$ \\
\hline $\mathrm{c} / \AA$ & $21.22584(18)$ \\
\hline$\alpha /{ }^{\circ}$ & 90 \\
\hline$\beta /{ }^{\circ}$ & 90 \\
\hline$\gamma /{ }^{\circ}$ & 90 \\
\hline Volume/ $\AA^{3}$ & $5562.50(9)$ \\
\hline $\mathrm{Z}$ & 8 \\
\hline$\rho_{\text {calcg/cm }}^{3}$ & 1.198 \\
\hline$\mu / \mathrm{mm}^{-1}$ & 0.599 \\
\hline $\mathrm{F}(000)$ & 2128.0 \\
\hline Crystal size/mm & \\
\hline Radiation & $0.2 \times 0.15 \times 0.02$ \\
\hline $2 \Theta$ range for data collection/ ${ }^{\circ}$ & $\mathrm{CuK \alpha}(\lambda=1.54184)$ \\
\hline Index ranges & 8.332 to 148.078 \\
\hline Reflections collected & $-18 \leq \mathrm{h} \leq 19,-21 \leq \mathrm{k} \leq 20,-15 \leq 1 \leq 26$ \\
\hline Independent reflections & 17912 \\
\hline Data/restraints/parameters & $5553\left[\mathrm{R}_{\text {int }}=0.0360, \mathrm{R}_{\text {sigma }}=0.0293\right]$ \\
\hline Goodness-of-fit on $\mathrm{F}^{2}$ & $5553 / 0 / 347$ \\
\hline Final R indexes $[\mathrm{I}>=2 \sigma(\mathrm{I})]$ & 1.020 \\
\hline Final $\mathrm{R}$ indexes [all data] & $\mathrm{R}_{1}=0.0422, \mathrm{wR}_{2}=0.1139$ \\
\hline Largest diff. peak/hole / e $\AA^{-3}$ & $\mathrm{R}_{1}=0.0484, \mathrm{wR}_{2}=0.1204$ \\
\hline & $0.27 /-0.24$ \\
\hline & \\
\hline
\end{tabular}




\section{(E) References}

[1] Mio, M. J.; Kopel, L. C.; Braun, J. B.; Gadzikwa, T. L.; Hull, K. L.; Brisbois, R. G.; Markworth, C. J.; Grieco, P. A. Org. Lett. 2002, 4, 3199.

[2] a) Li, T.; Zhou, C.; Yan, X.; Wang, J. Angew. Chem. Int. Ed. 2018, 57, 4048. b) Fukui, Y.; Liu, P.; Liu, Q.; He, Z.-T.; Wu, N.-Y.; Tian, P.; Lin, G.-Q. J. Am. Chem. Soc. 2014, 136, 15607.

[3] a) Liu, Y.; Zhou, C.; Xiong, M.; Jiang, J.; Wang, J. Org. Lett. 2018, 20, 5889. b) Tang, Y.; Chen, Q.; Liu, X.; Wang, G.; Lin, L.; Feng, X. Angew. Chem. Int. Ed. 2015, 54, 9512. c) Lee, E. C.; Fu, G. C. J. Am. Chem. Soc. 2007, 129, 12066.

[4] Mamillapalli, N. C.; Sekar, G. RSC Adv. 2014, 4, 61077.

[5] Dolomanov, O. V.; Bourhis, L. J.; Gildea, R. J.; Howard, J. A. K.; Puschmann, H. J. Appl. Crystallogr. 2009, 42, 339.

[6] Sheldrick, G. M. Acta Crystallogr C Struct Chem 2015, 71, 3.

(F) Copies of ${ }^{1} \mathrm{H}$ and ${ }^{13} \mathrm{C}$ NMR Spectra 

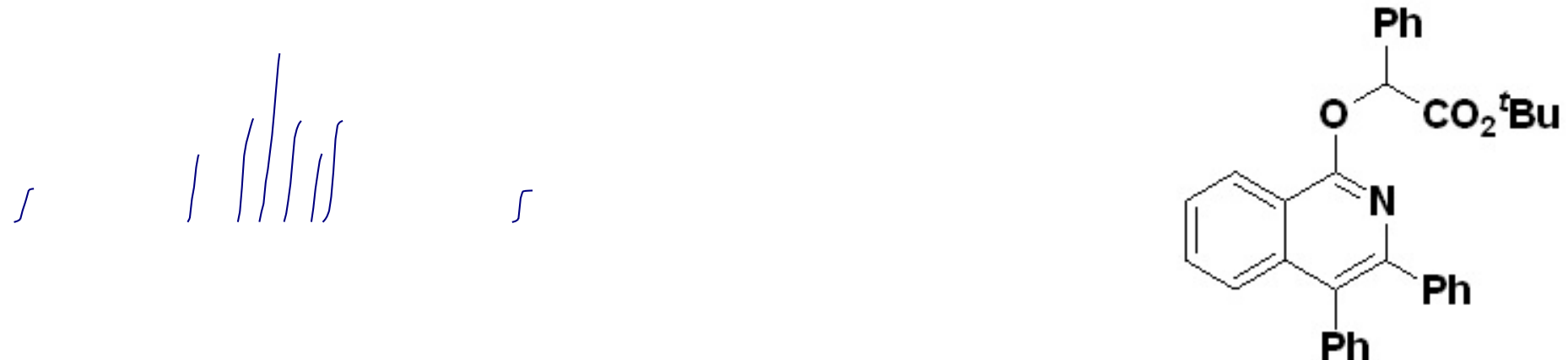

4aaa

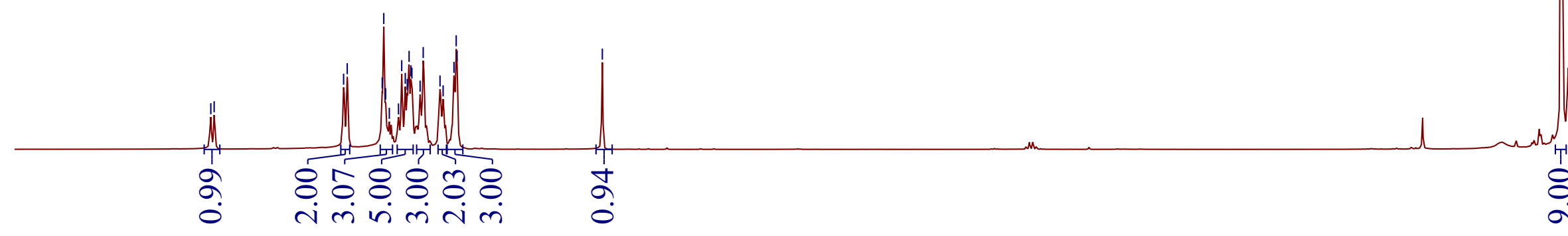

\begin{tabular}{lllllllllllllllllllll}
\hline .5 & 9.0 & 8.5 & 8.0 & 7.5 & 7.0 & 6.5 & 6.0 & 5.5 & $\begin{array}{c}5.0 \\
\mathrm{f} 1(\mathrm{ppm})\end{array}$ & 4.5 & 4.0 & 3.5 & 3.0 & 2.5 & 2.0 & 1.5 & 1.0 & 0.5 & 0.0
\end{tabular}



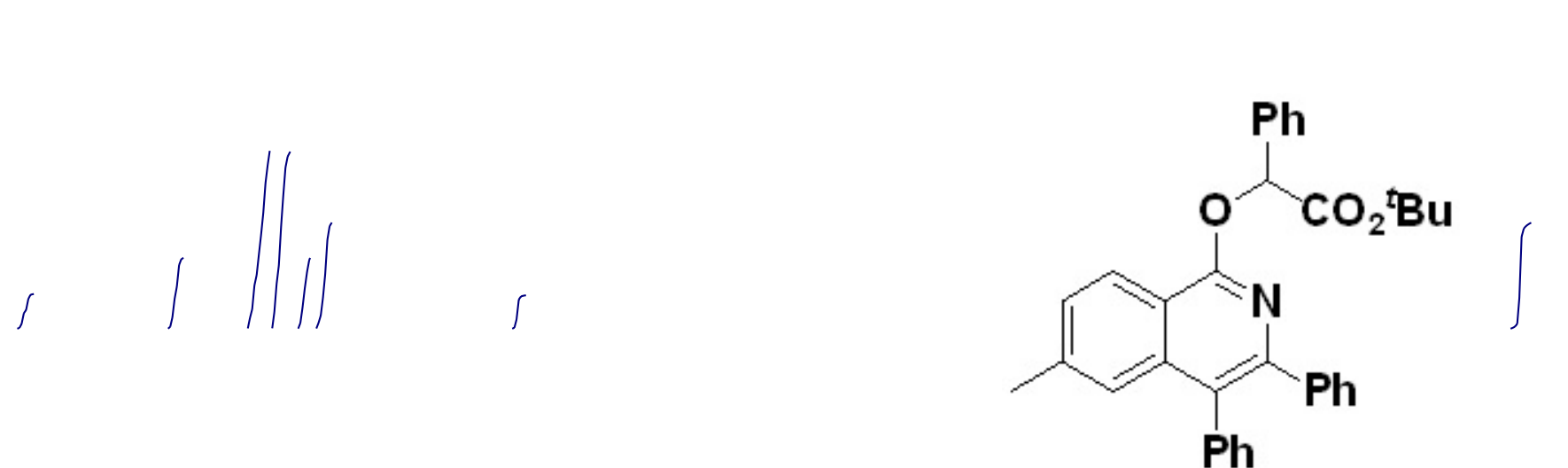

4baa

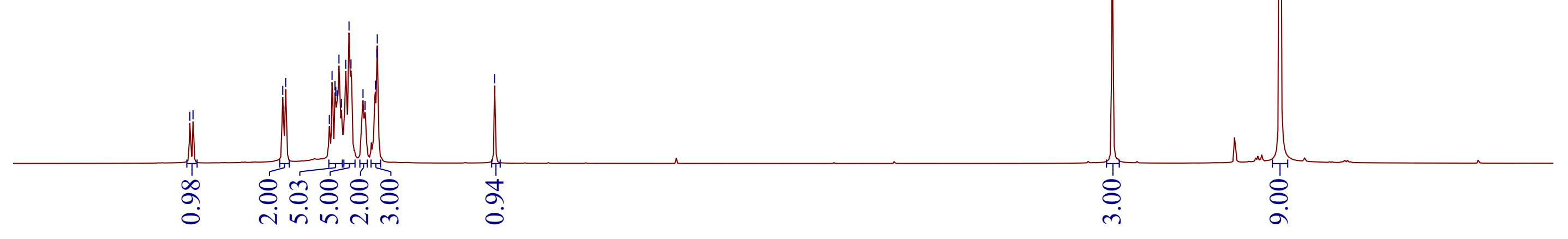

$8.5 \quad 8.0 \quad 7.5$

$\begin{array}{ll}7.0 & 6.5\end{array}$

$6.0 \quad 5.5$



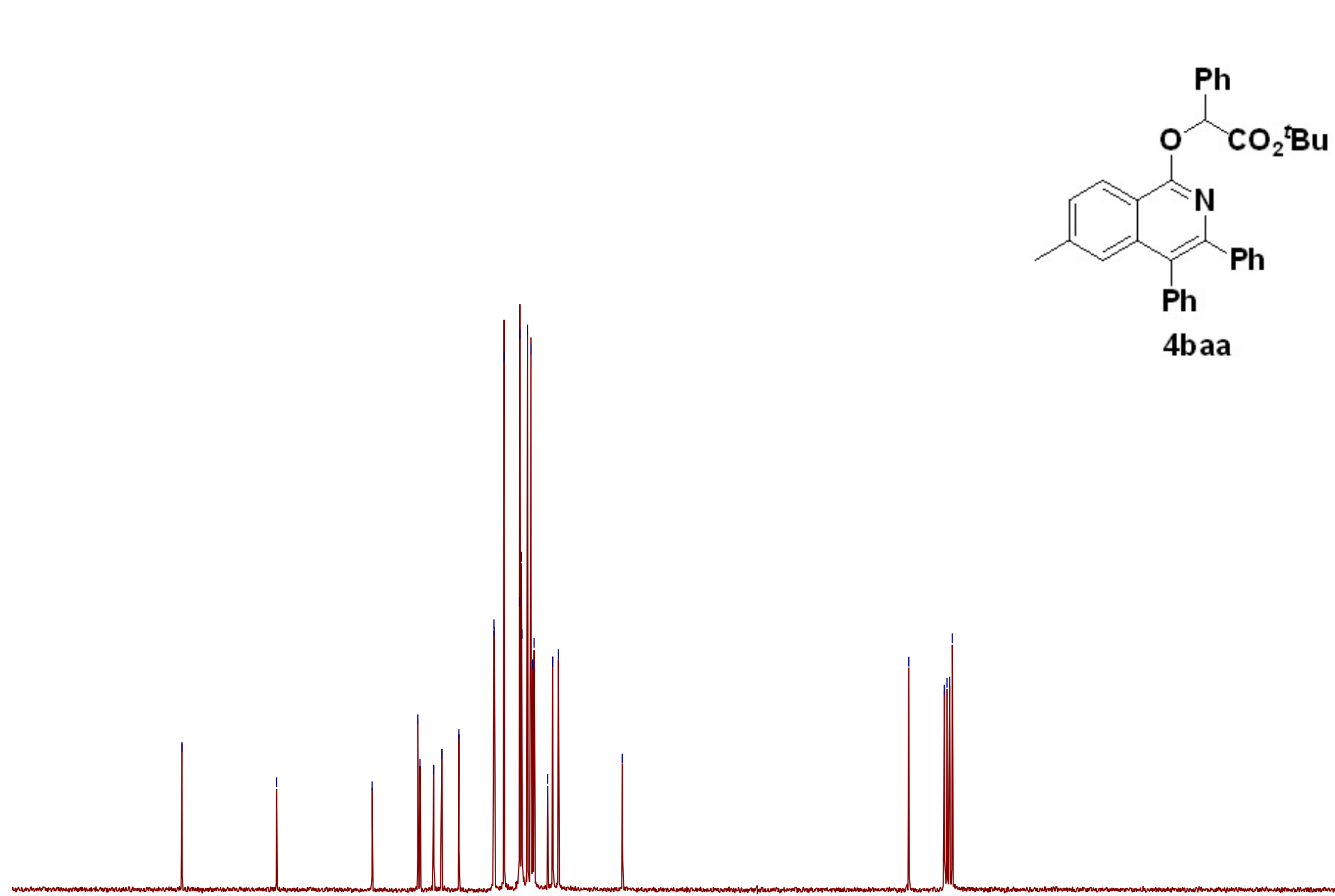

4baa 
4caa

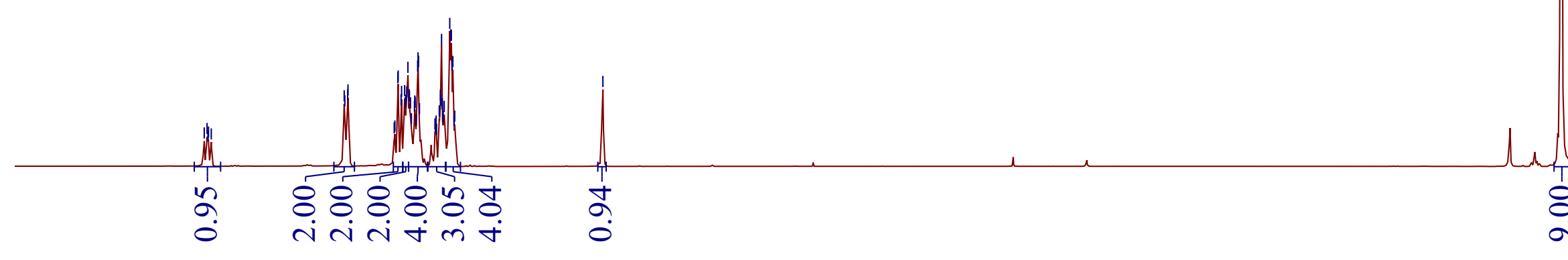

$\begin{array}{ccccccccccccccccccc}9.0 & 8.5 & 8.0 & 7.5 & 7.0 & 6.5 & 6.0 & 5.5 & \begin{array}{c}5.0 \\ \mathrm{f} 1(\mathrm{ppm})\end{array} & \begin{array}{c}4.5 \\ 4.0\end{array} & 3.5 & 3.0 & 2.5 & 2.0 & 1.5 & 1.0 & 0.5 & 0.0 & -0 .\end{array}$



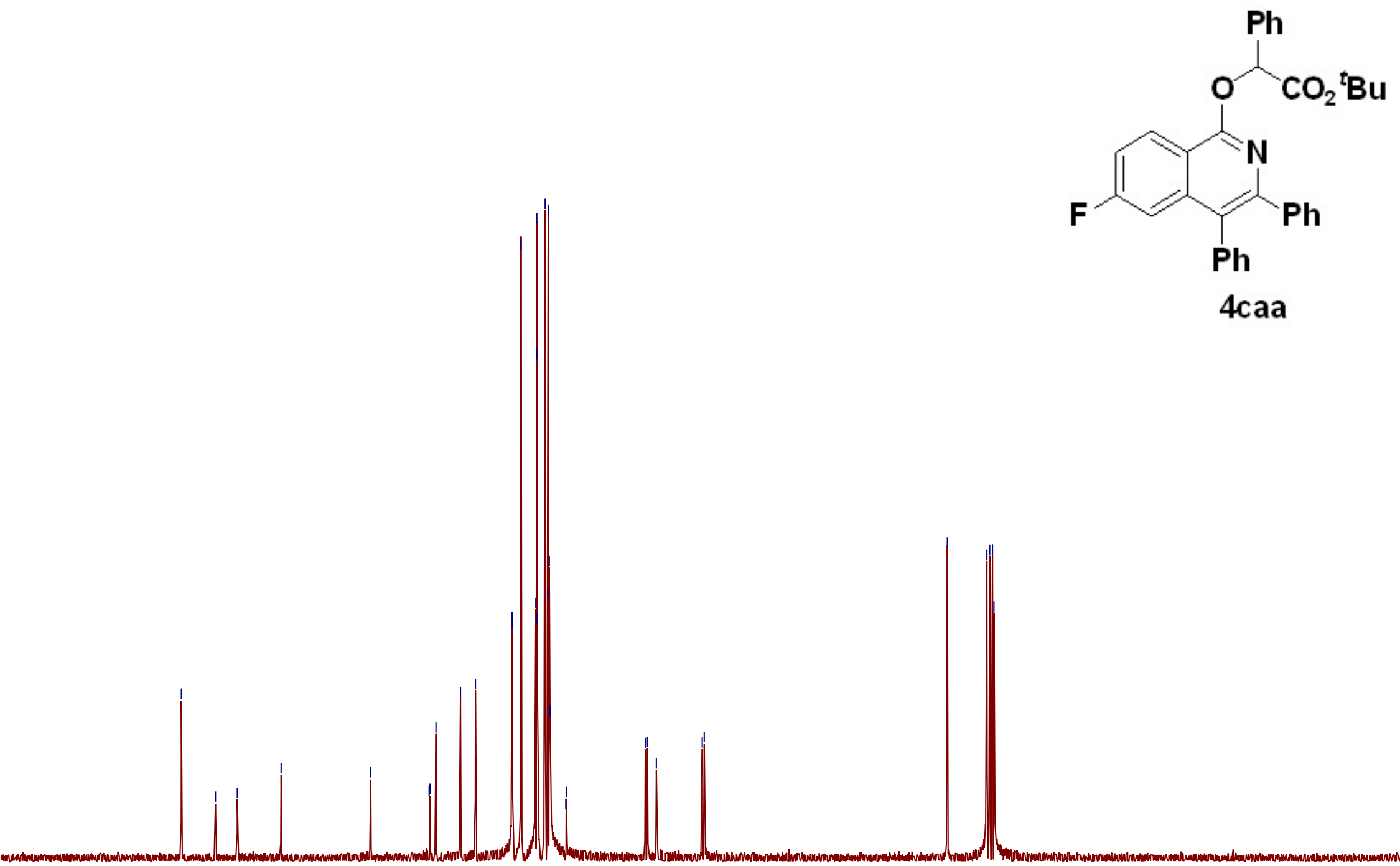

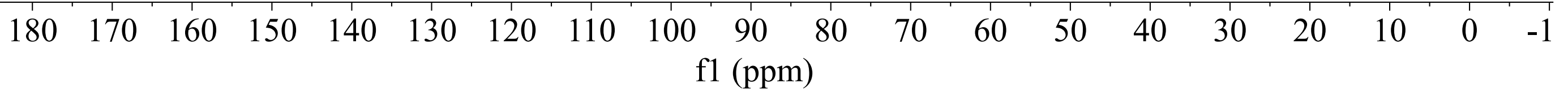




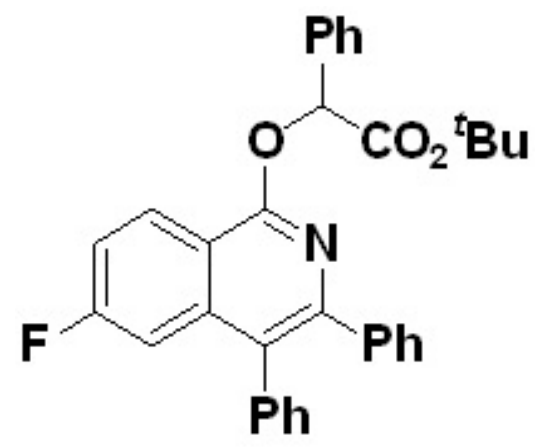

4caa

$\begin{array}{ccccccccccccccccccccc}100 & 80 & 60 & 40 & 20 & 0 & -20 & -40 & -60 & -80 & -100 & -120 & -140 & -160 & -180 & -200 & -220 & -240 & -260 & -280 & -300\end{array}$



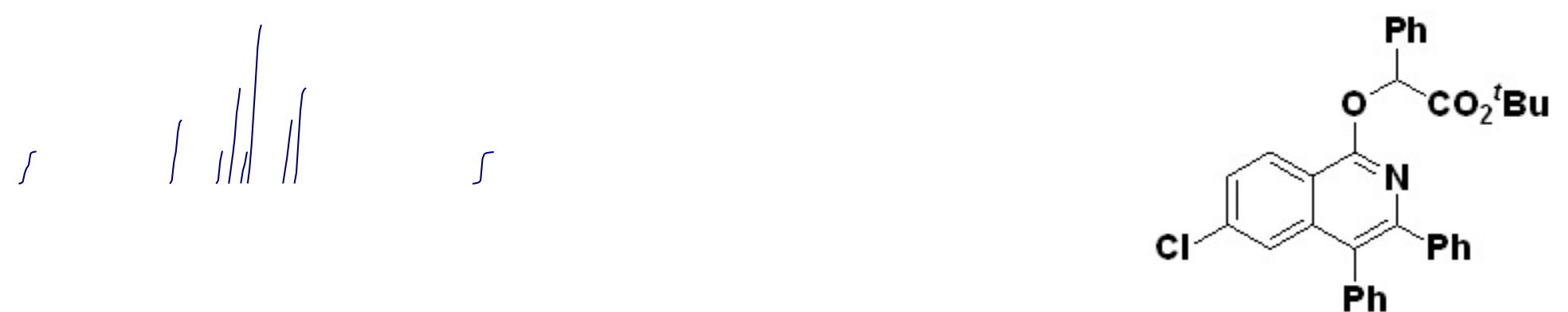

4daa

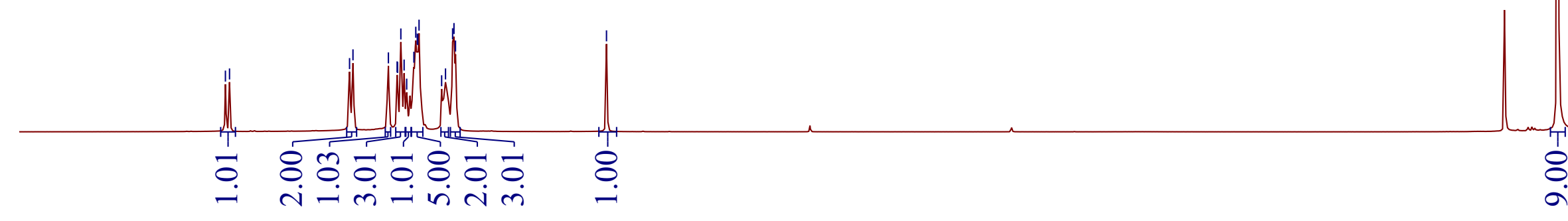

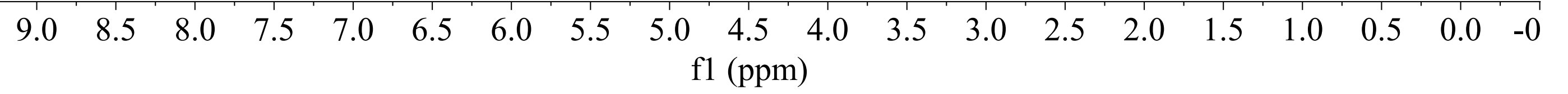




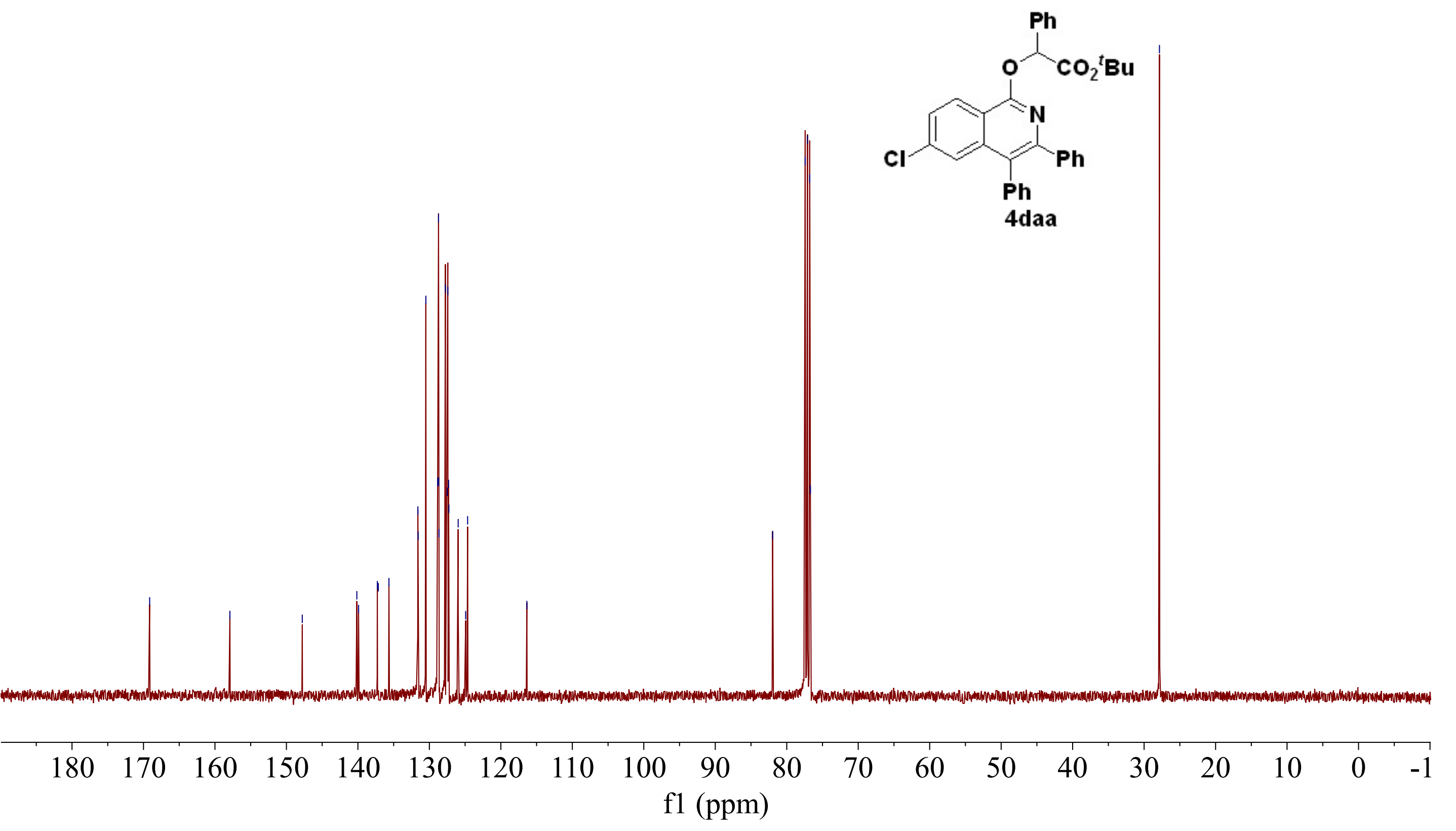




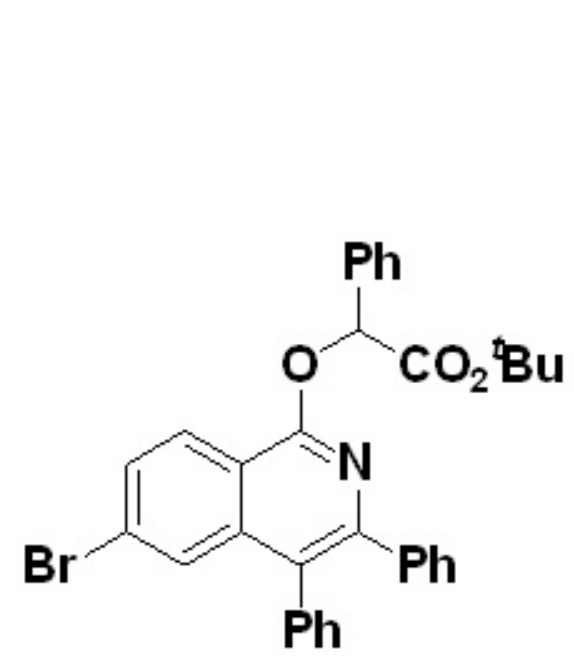

4eaa

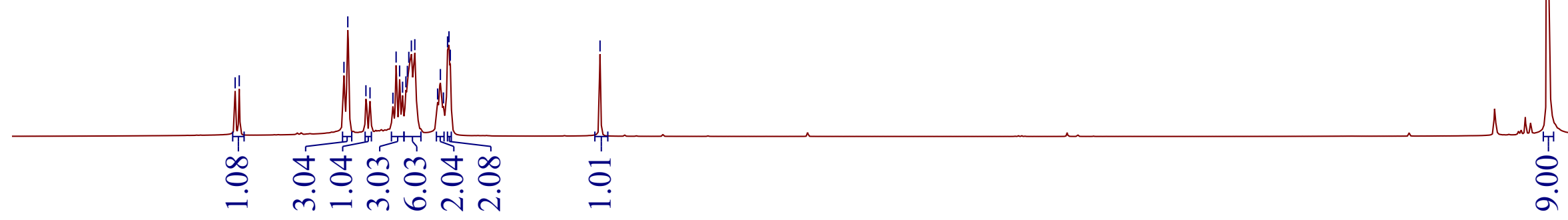

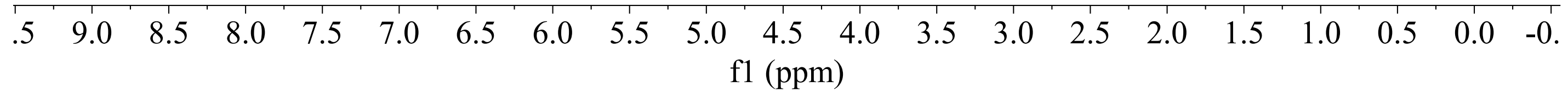




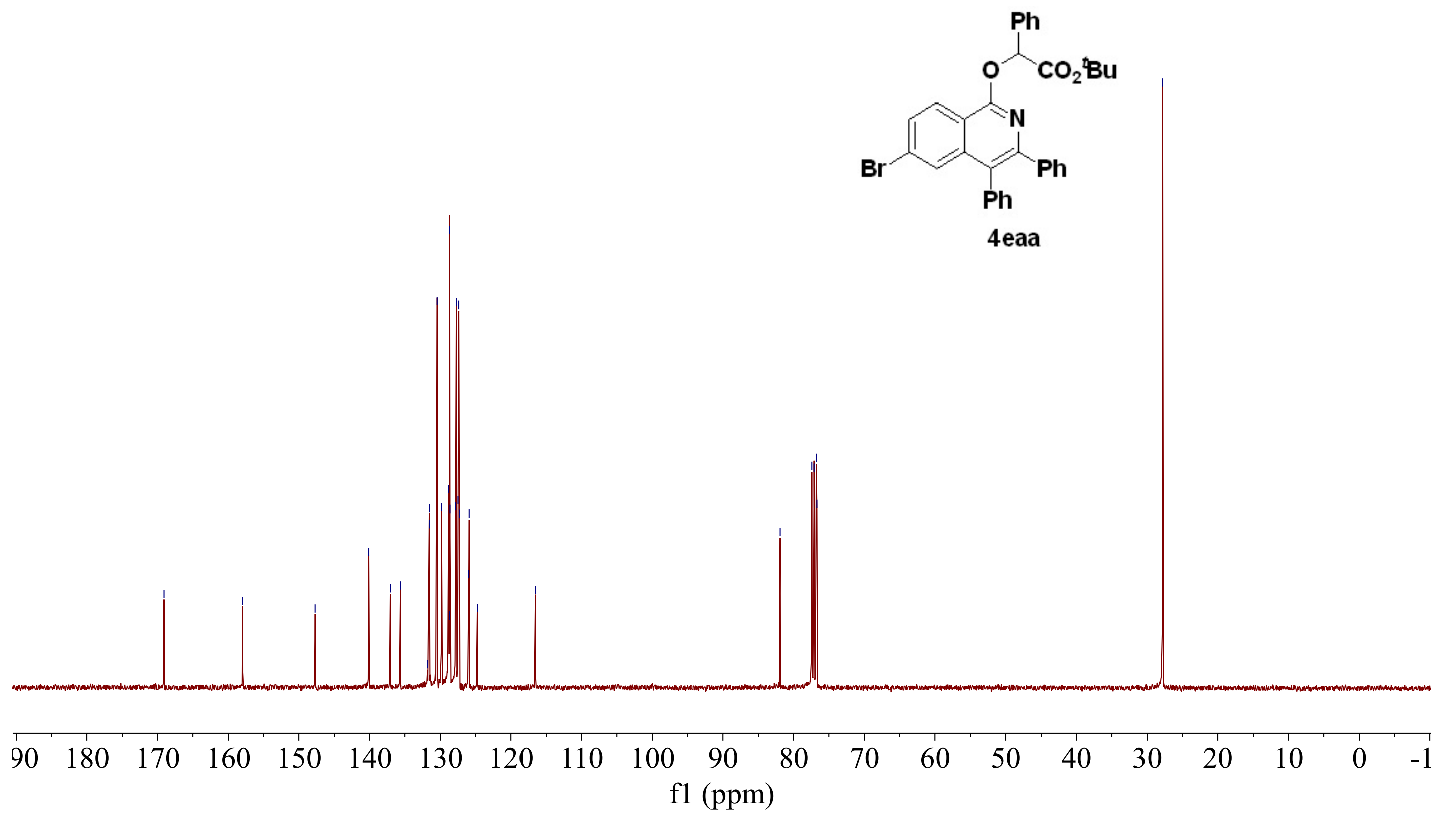




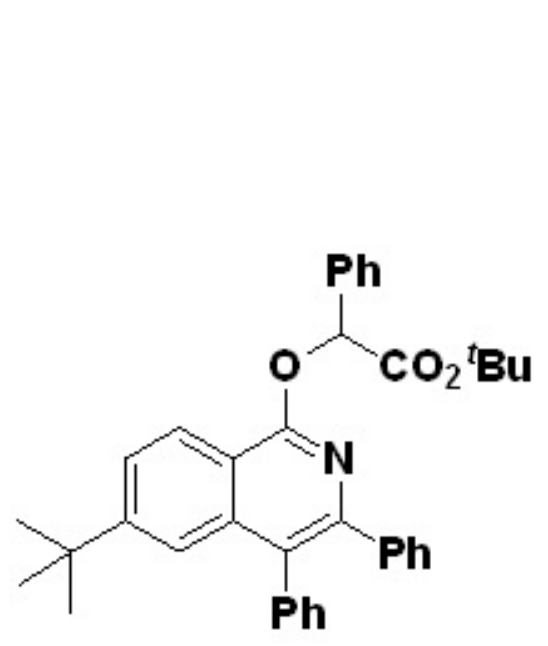

4faa

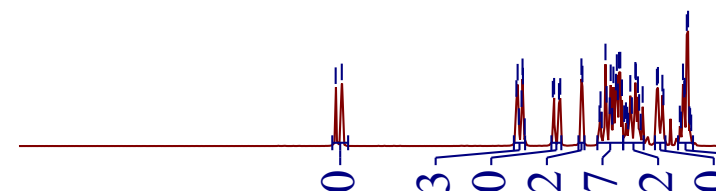

\&

$\therefore$

$\begin{array}{lllll}8.5 & 8.0 & 7.5 & 7.0 & 6.5\end{array}$

6.05 .5

$\begin{array}{llll}5.0 & 4.5 & 4.0 & 3.5 \\ \mathrm{fl}(\mathrm{ppm}) & \end{array}$ 


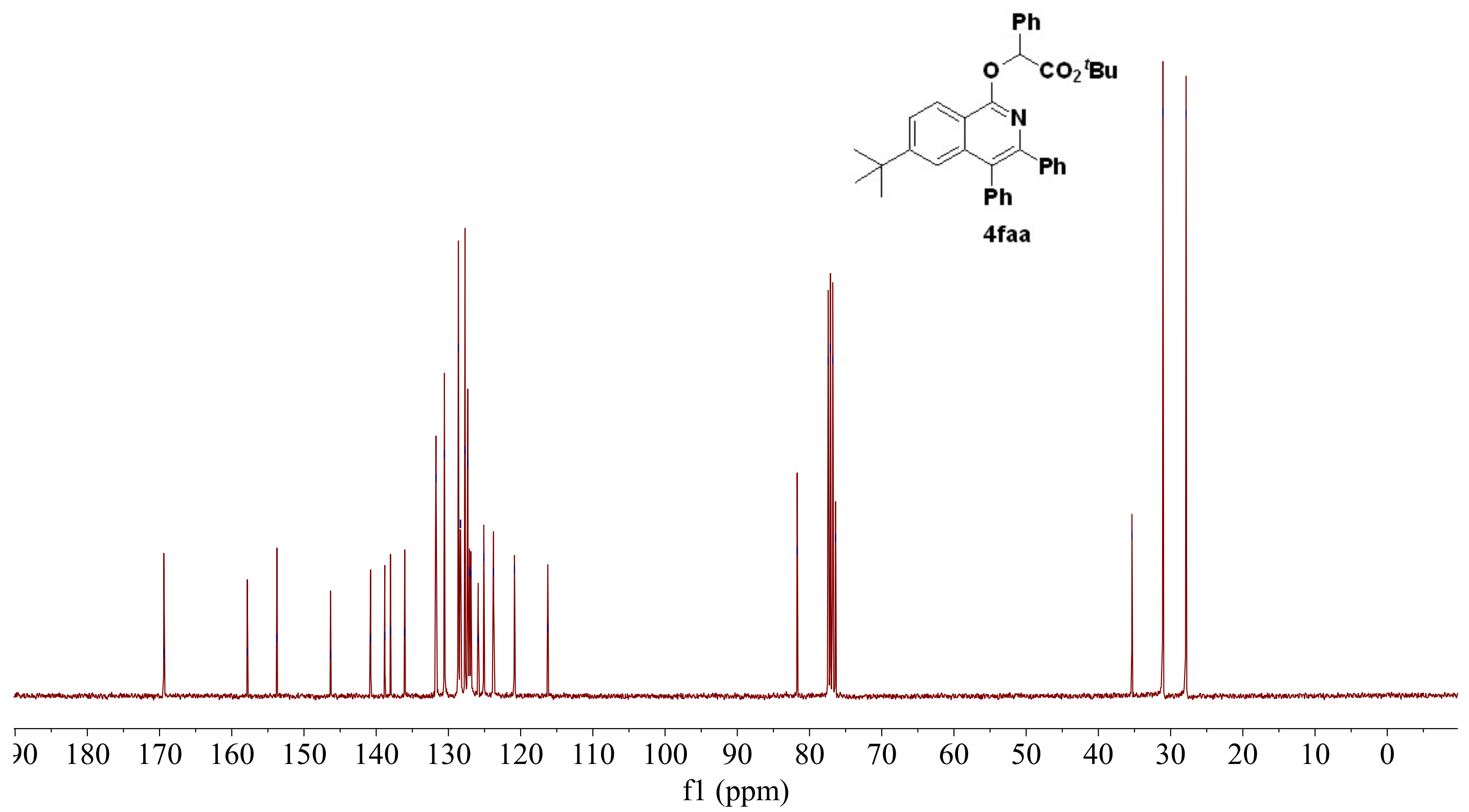



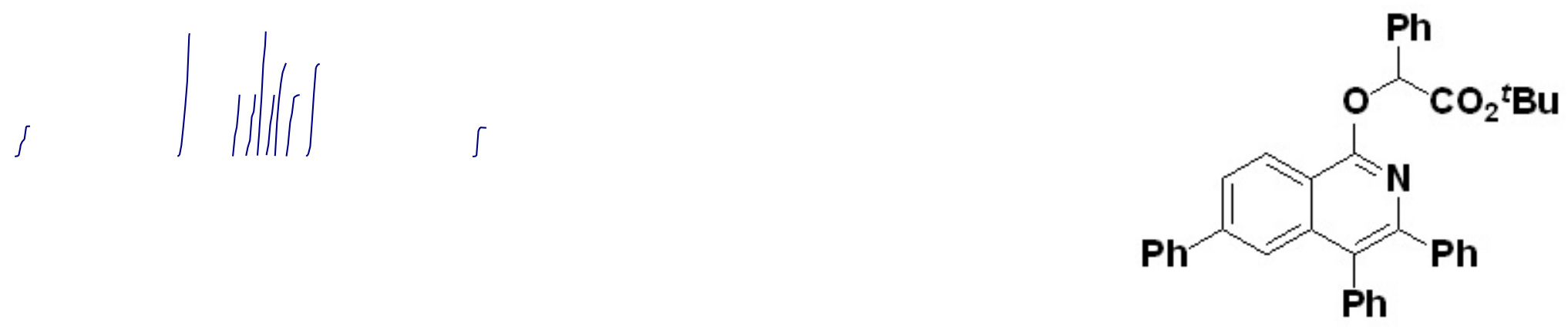

4gaa

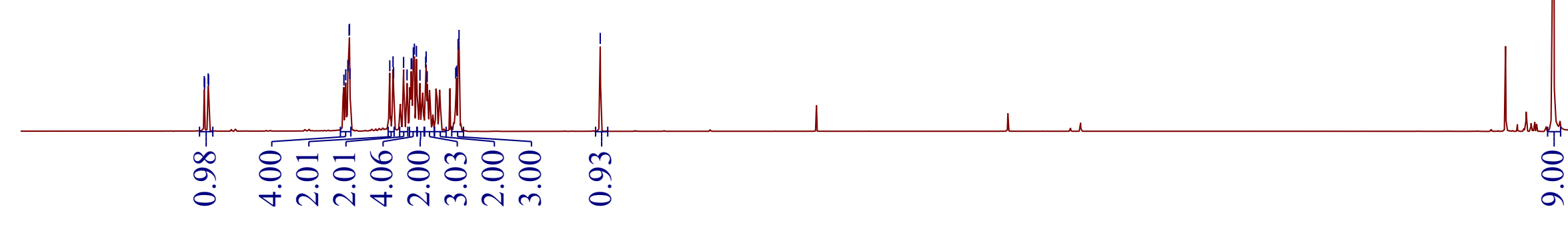

\begin{tabular}{lllllllllllllllllllll}
\hline .5 & 9.0 & 8.5 & 8.0 & 7.5 & 7.0 & 6.5 & 6.0 & 5.5 & 5.0 & $\begin{array}{c}4.5 \\
\text { fl }(\mathrm{ppm})\end{array}$ & 4.0 & 3.5 & 3.0 & 2.5 & 2.0 & 1.5 & 1.0 & 0.5 & 0.0 & -0.
\end{tabular}



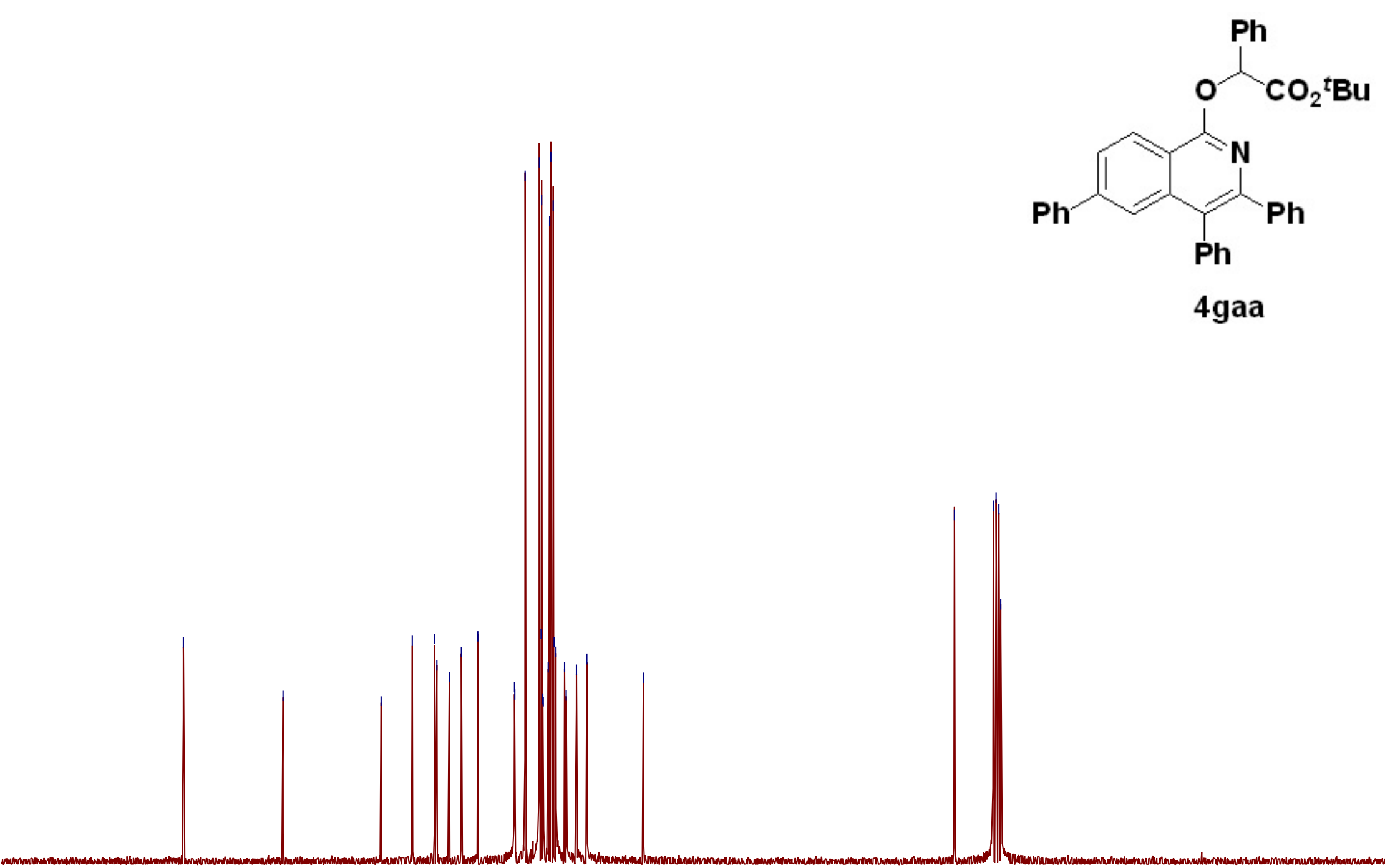

4gaa 

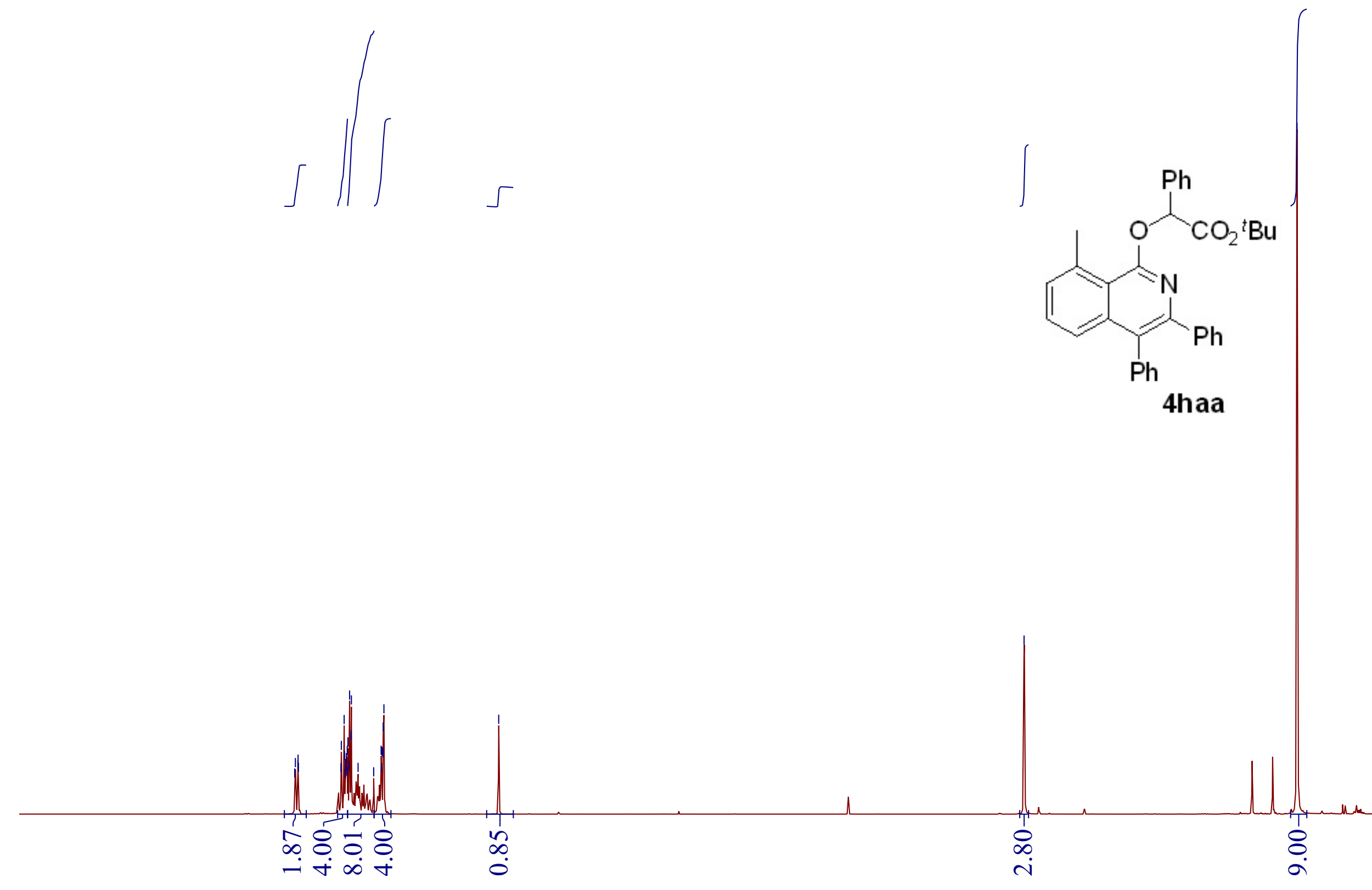

$\begin{array}{lll}8.5 & 8.0 & 7.5\end{array}$

7.0

6.5

$6.0 \quad 5.5$

5.04 .5 f1 (ppm) 

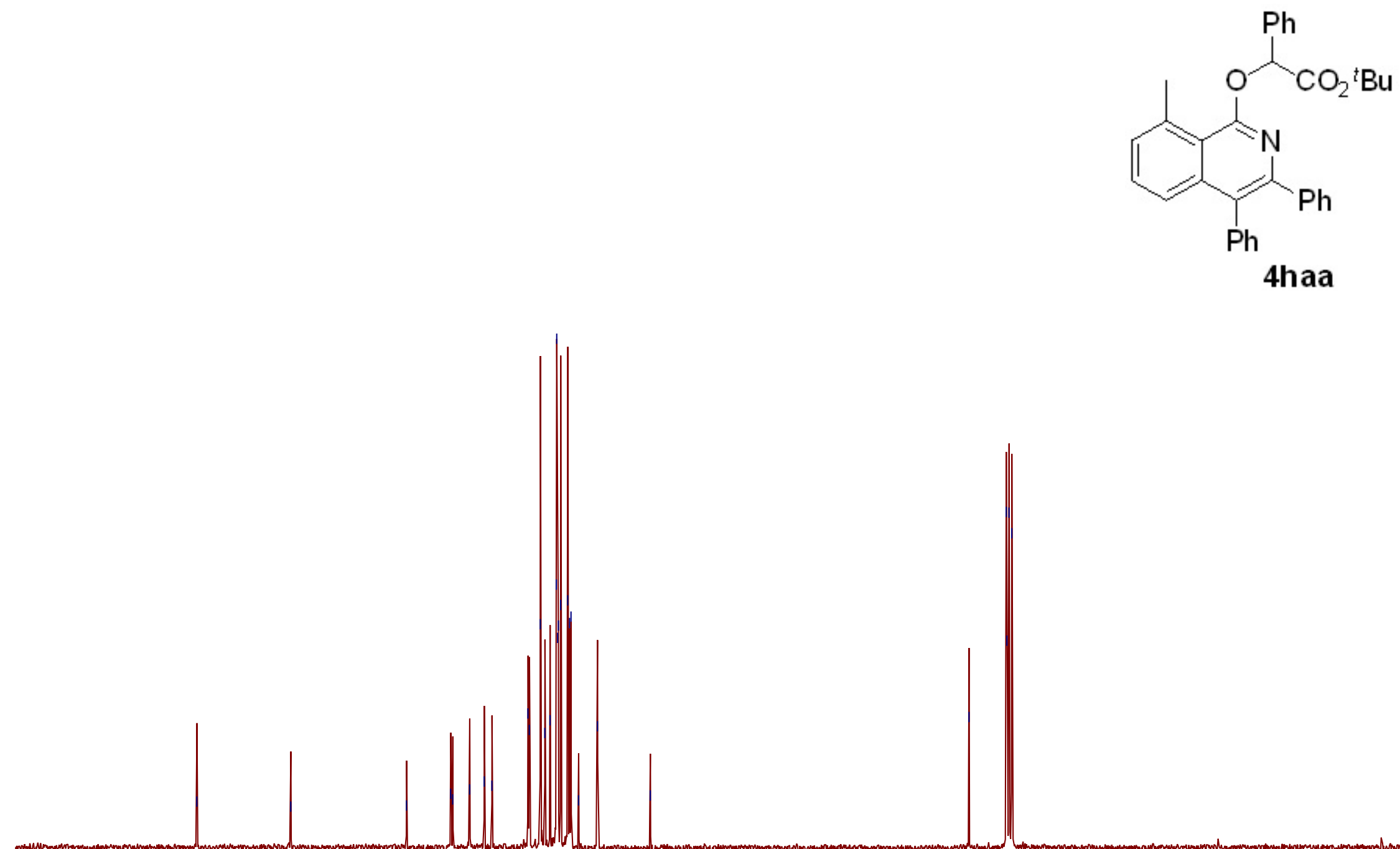

4haa

$30 \quad 180 \quad 170 \quad 160 \quad 150$

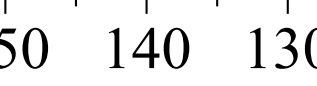

120

10 


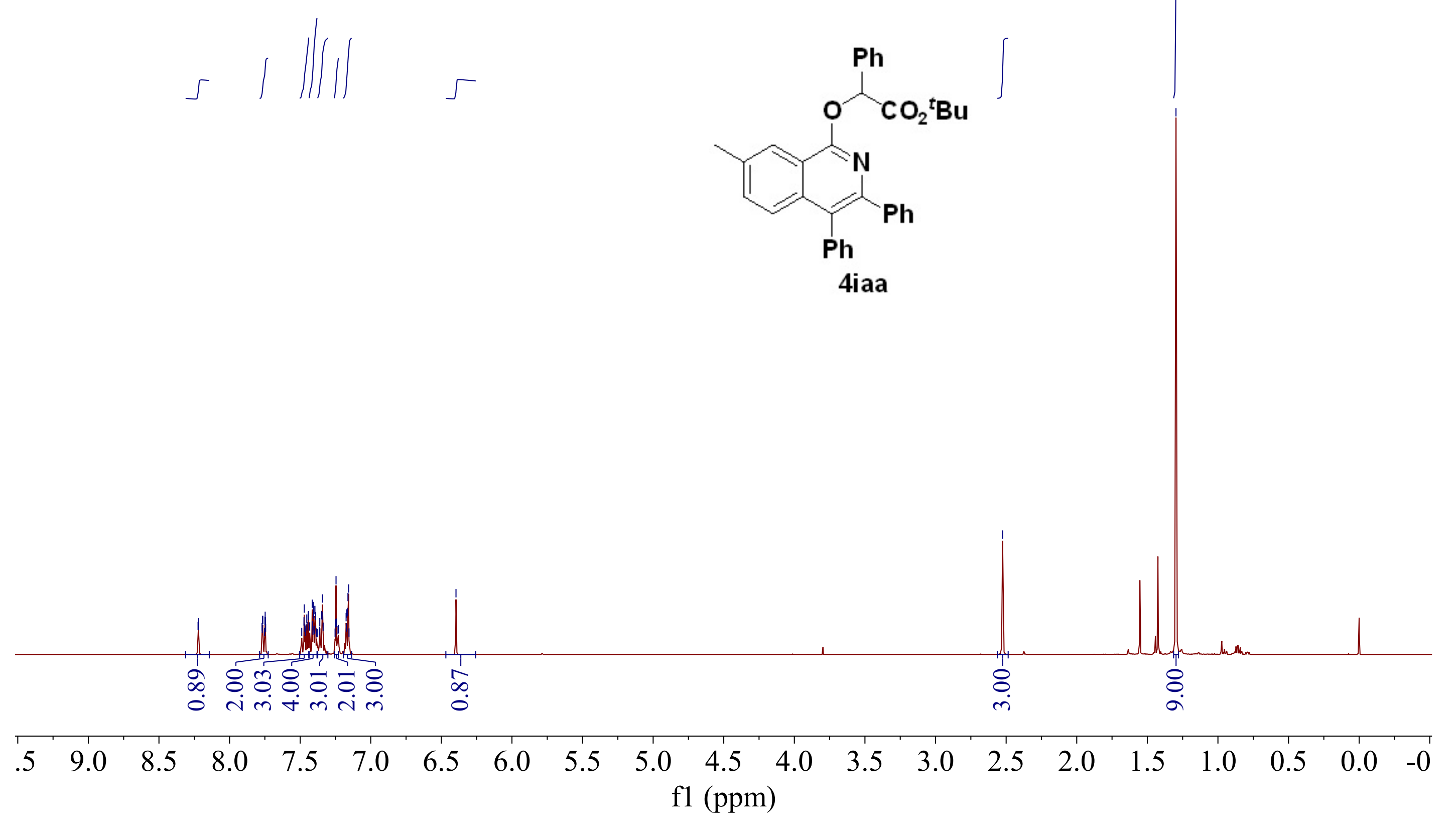




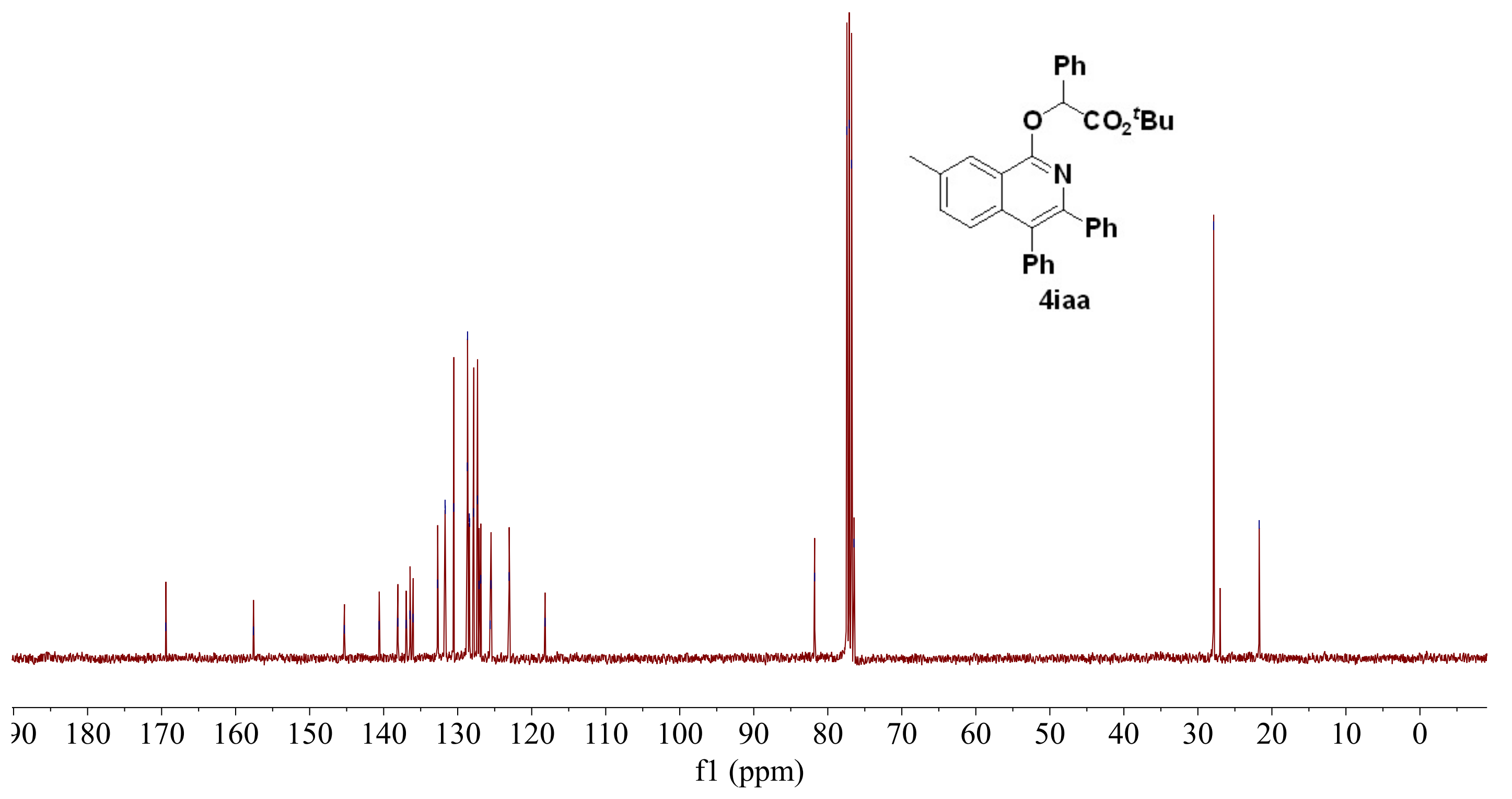



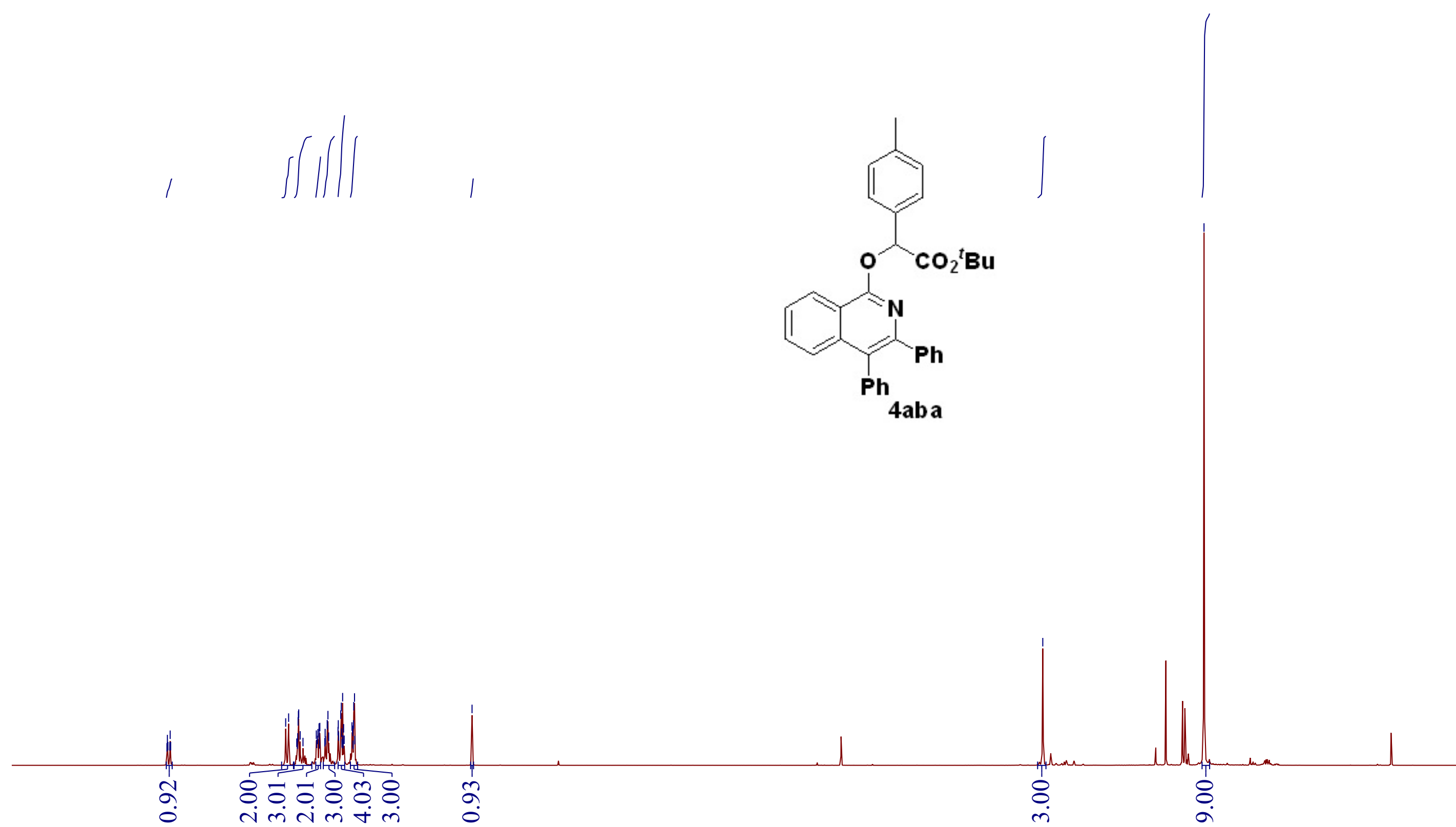

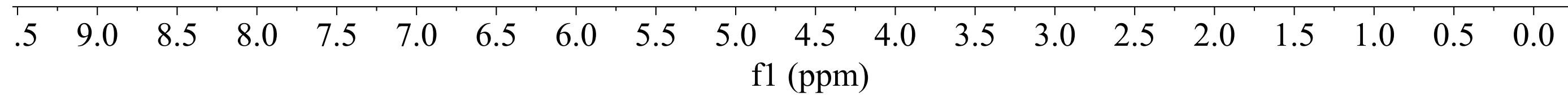




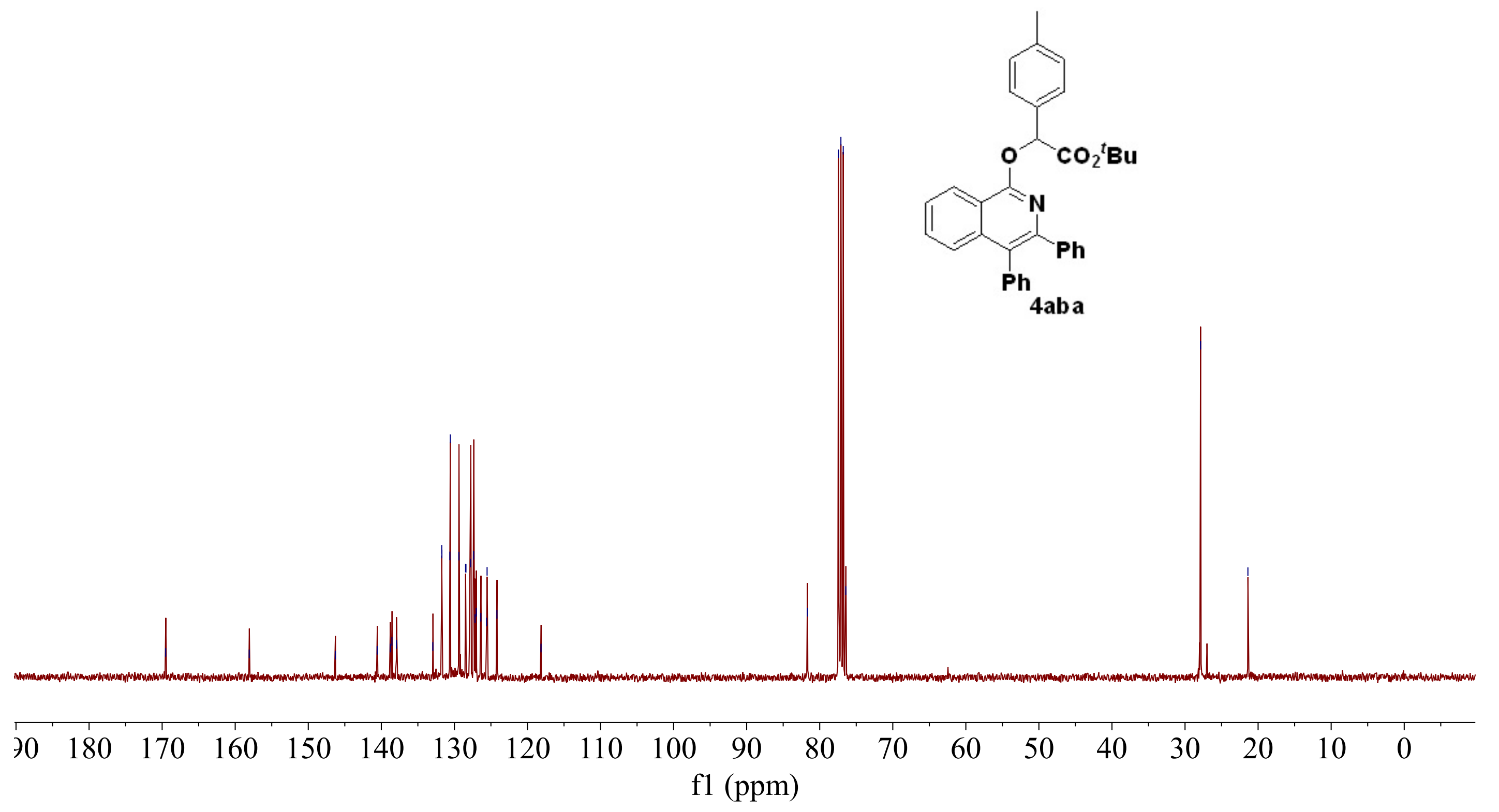




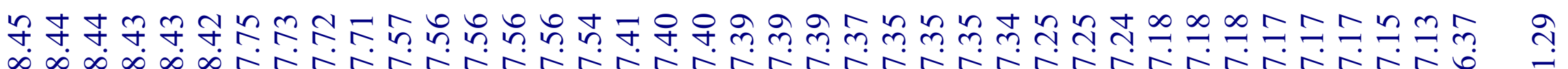

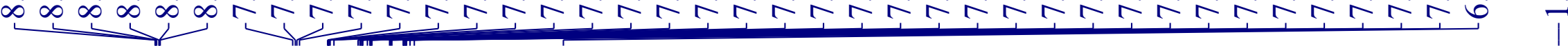

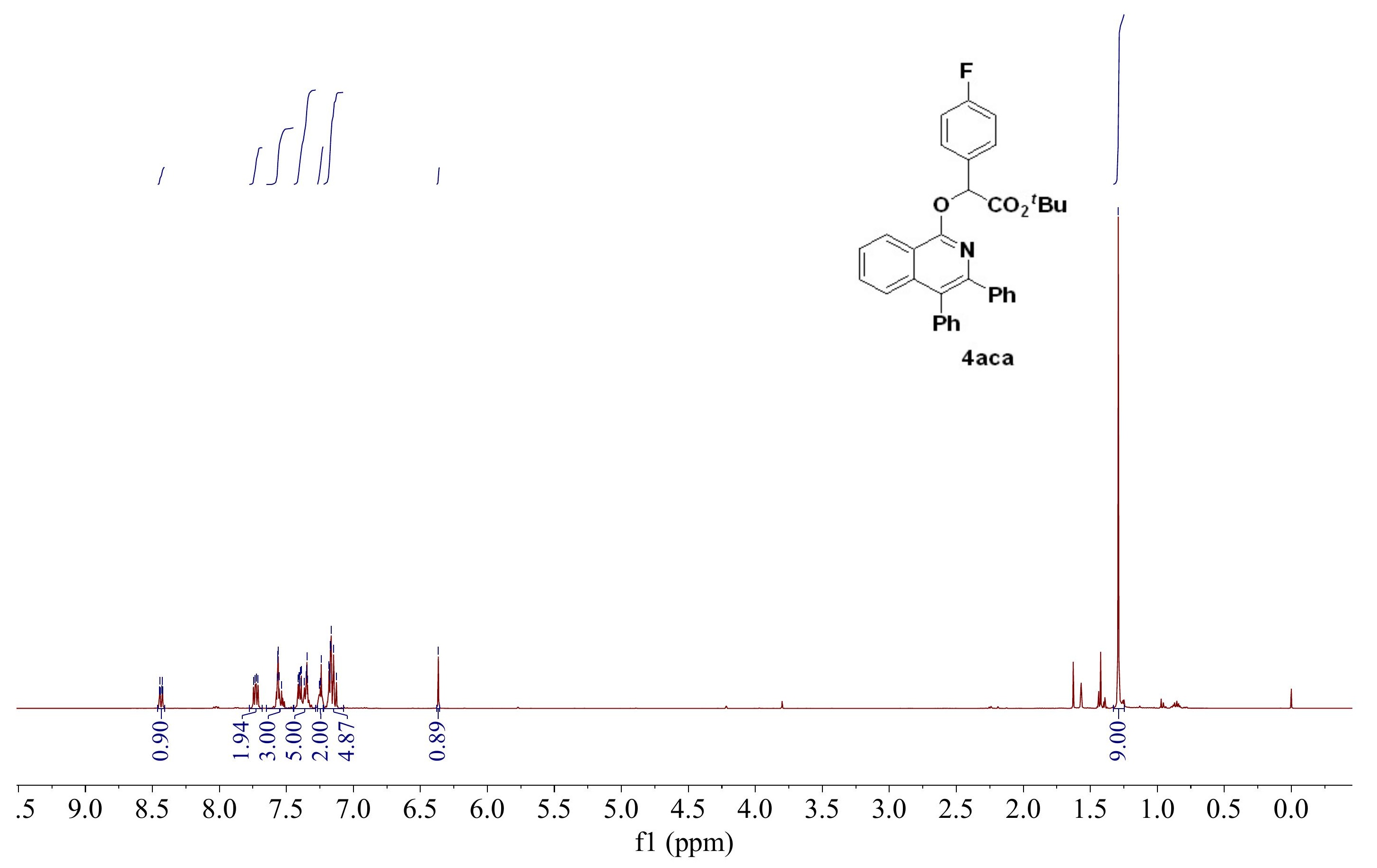




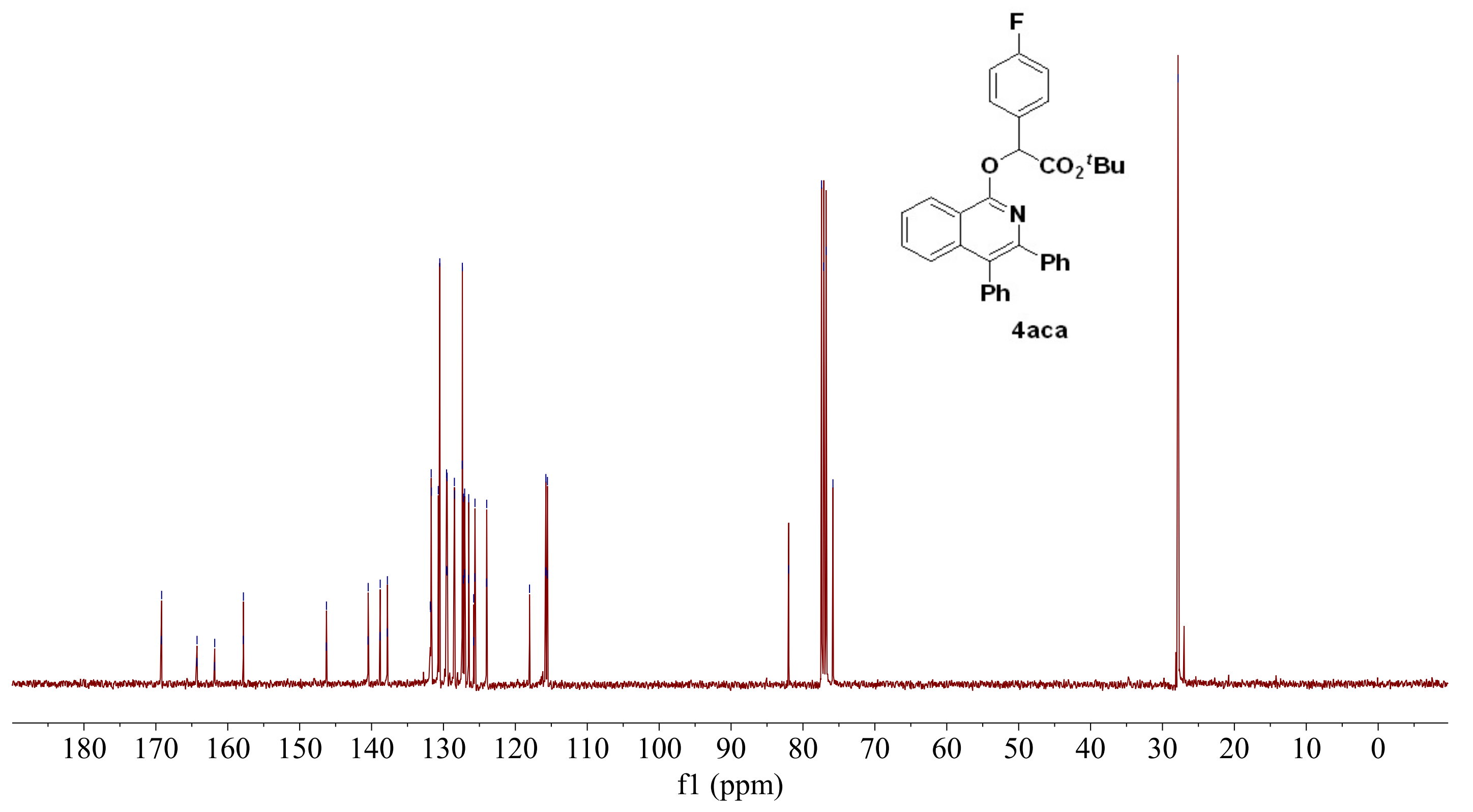




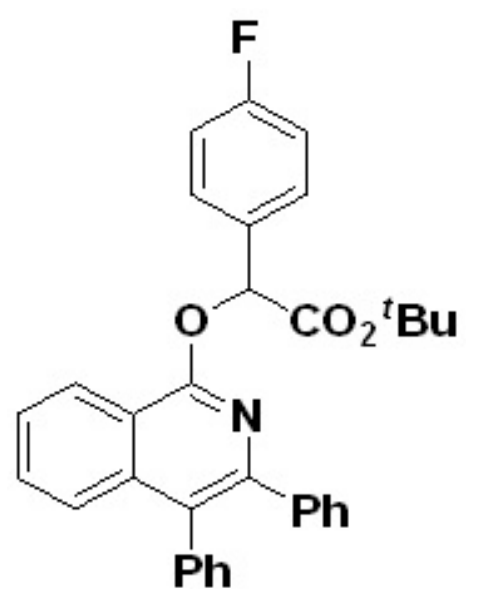

4aca

$\begin{array}{ccccccccccccccccccccccc}100 & 80 & 60 & 40 & 20 & 0 & -20 & -40 & -60 & -80 & -100 & -120 & -140 & -160 & -180 & -200 & -220 & -240 & -260 & -280 & -300\end{array}$




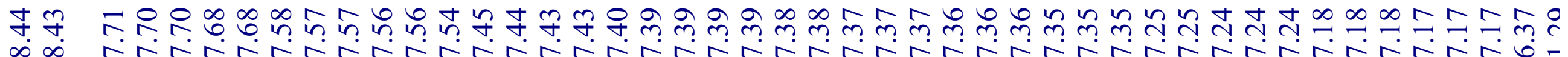

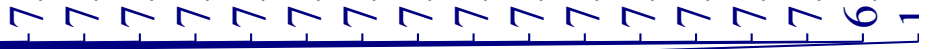

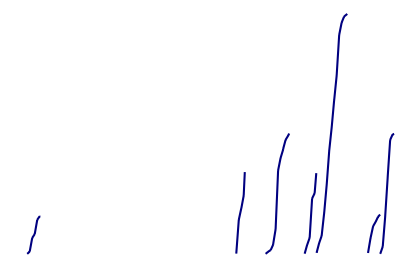

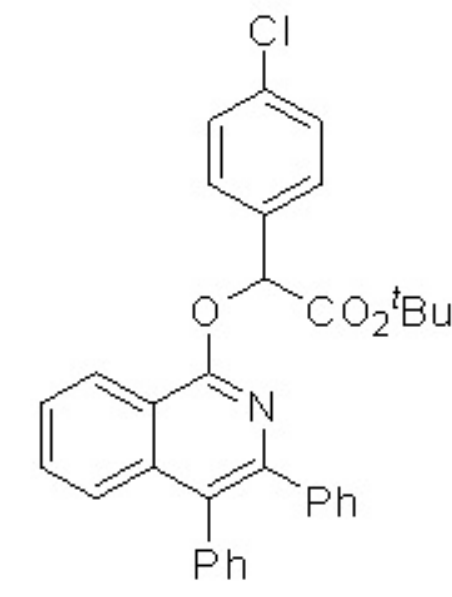

4ada
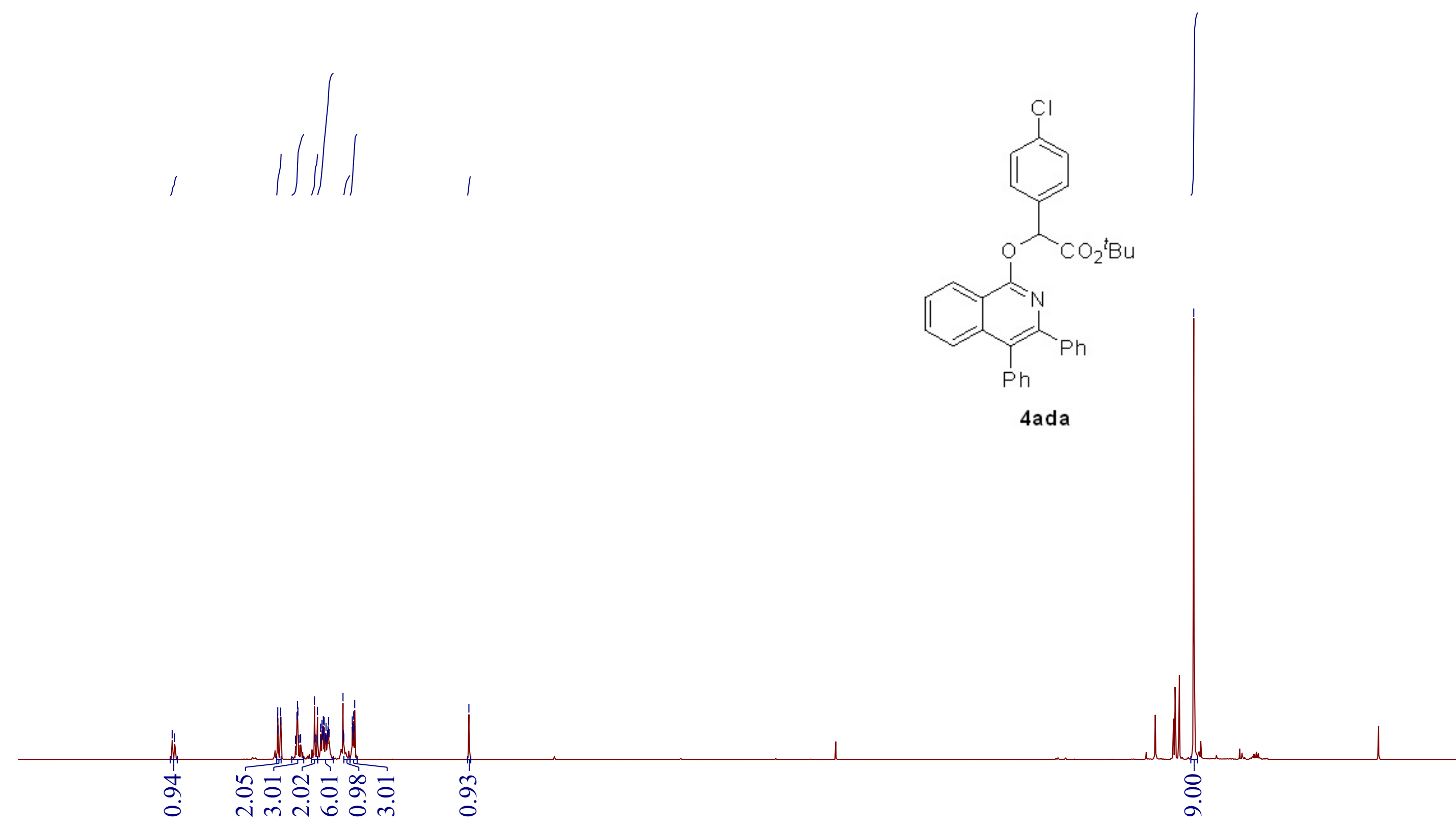

\begin{tabular}{llllllllllllllllllllll}
\hline .5 & 9.0 & 8.5 & 8.0 & 7.5 & 7.0 & 6.5 & 6.0 & 5.5 & 5.0 & 4.5 & 4.0 & 3.5 & 3.0 & 2.5 & 2.0 & 1.5 & 1.0 & 0.5 & 0.0 & -0. \\
$\mathrm{f} 1(\mathrm{ppm})$
\end{tabular}




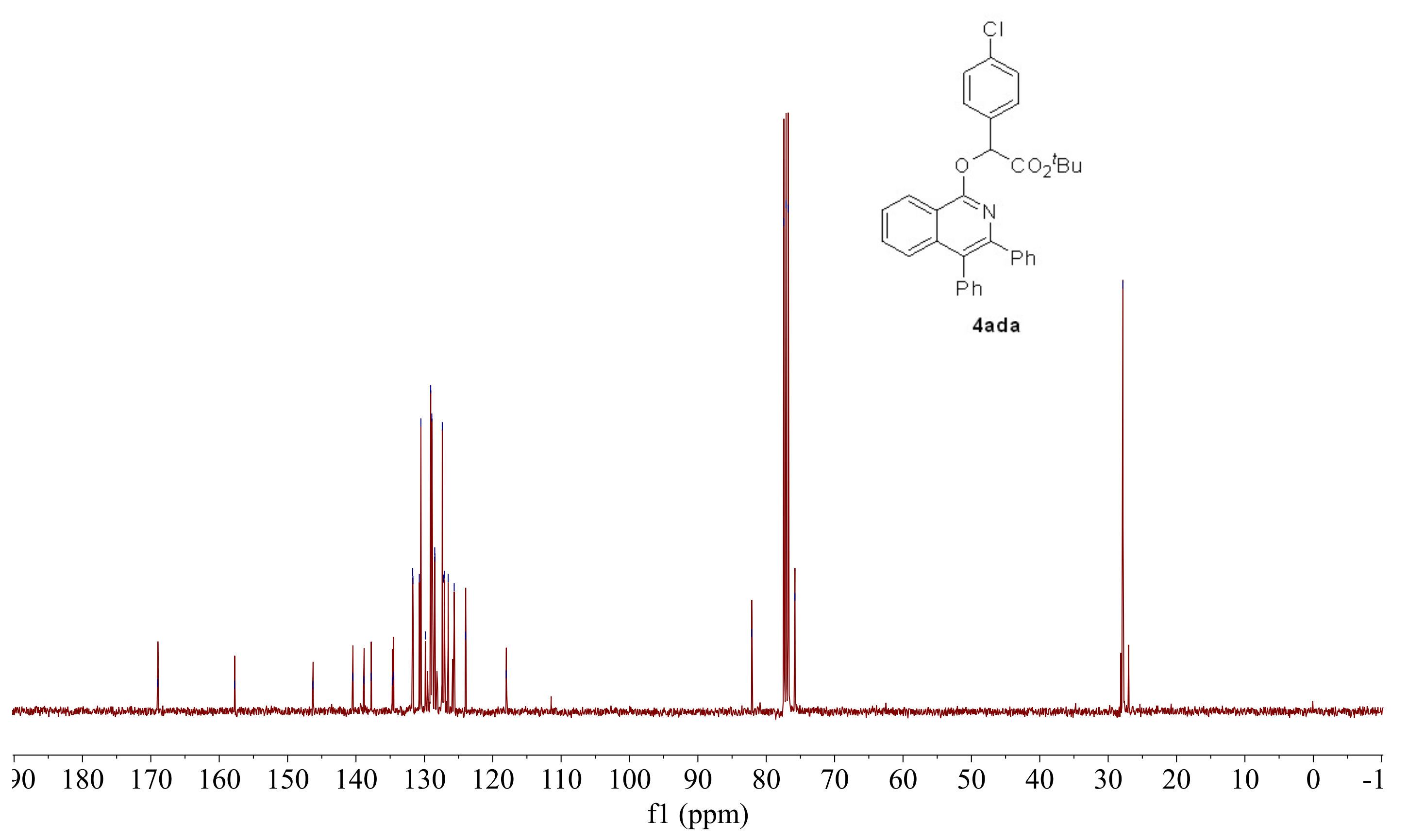



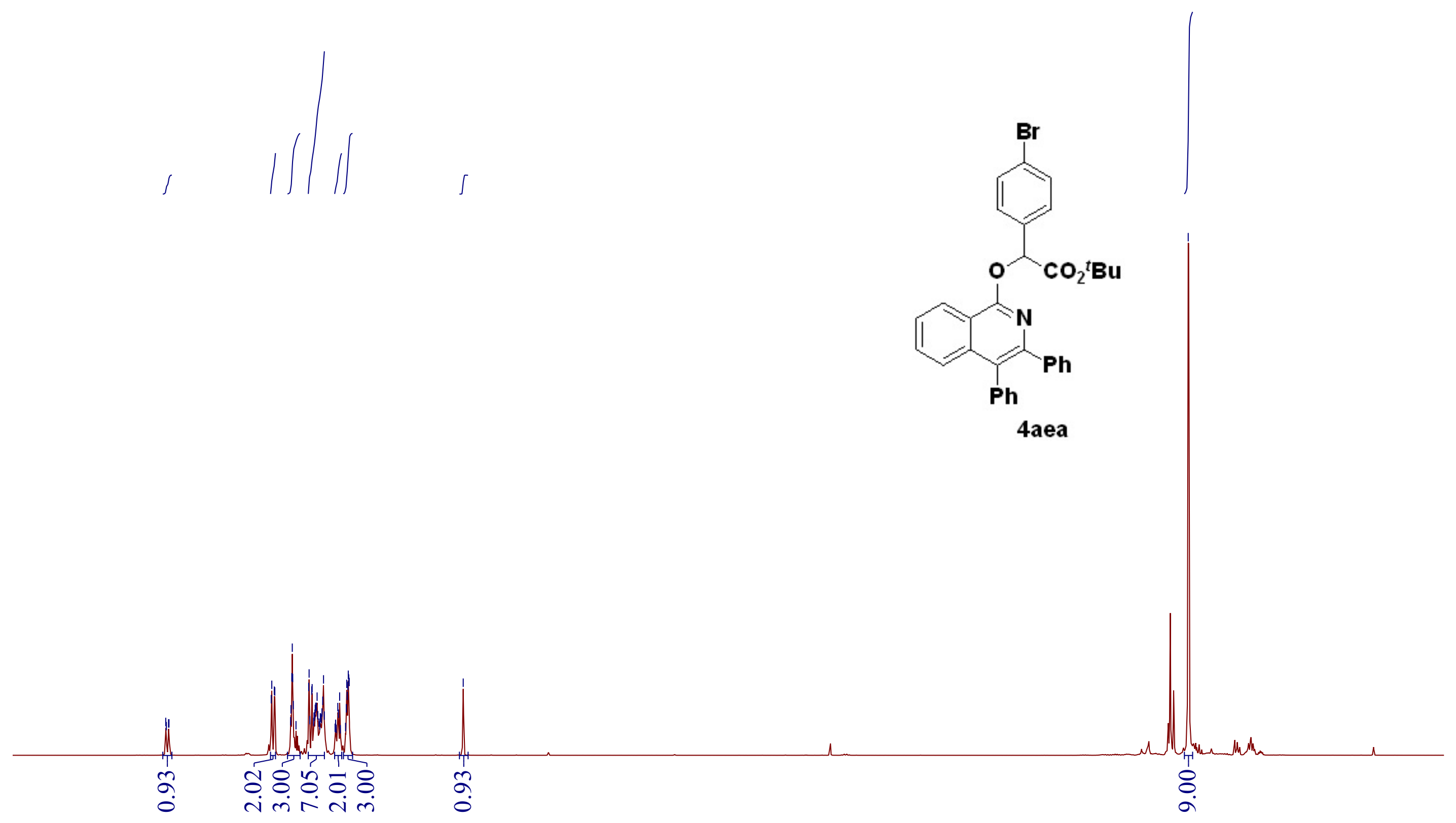

$\begin{array}{llll}8.5 & 8.0 & 7\end{array}$

7.06 .5

6.0

5.5

5.04 .5

f1 (ppm) 

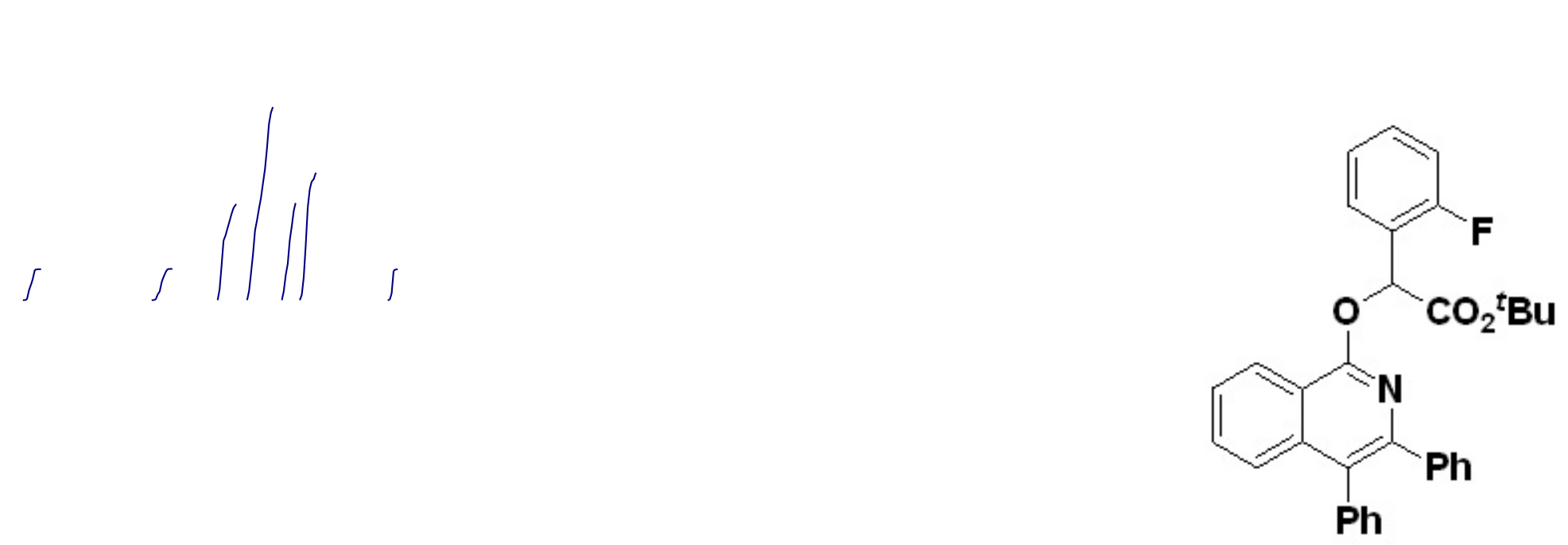

4afa

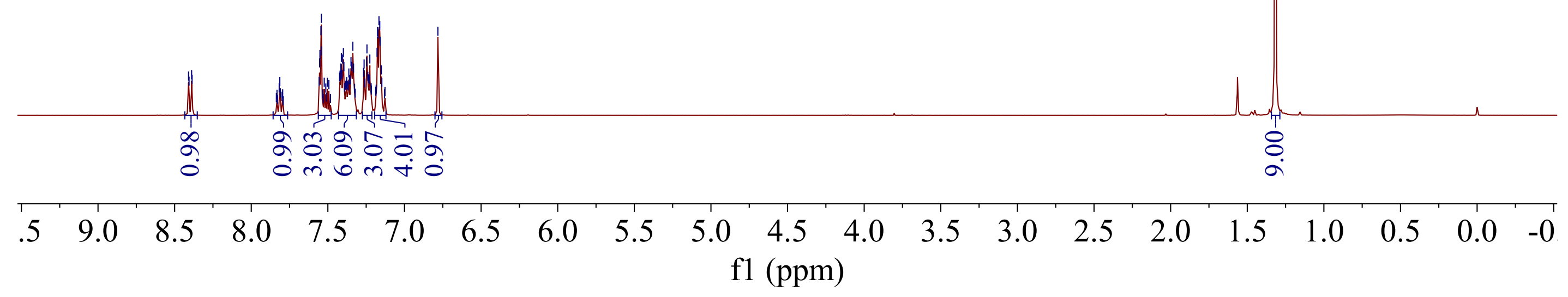




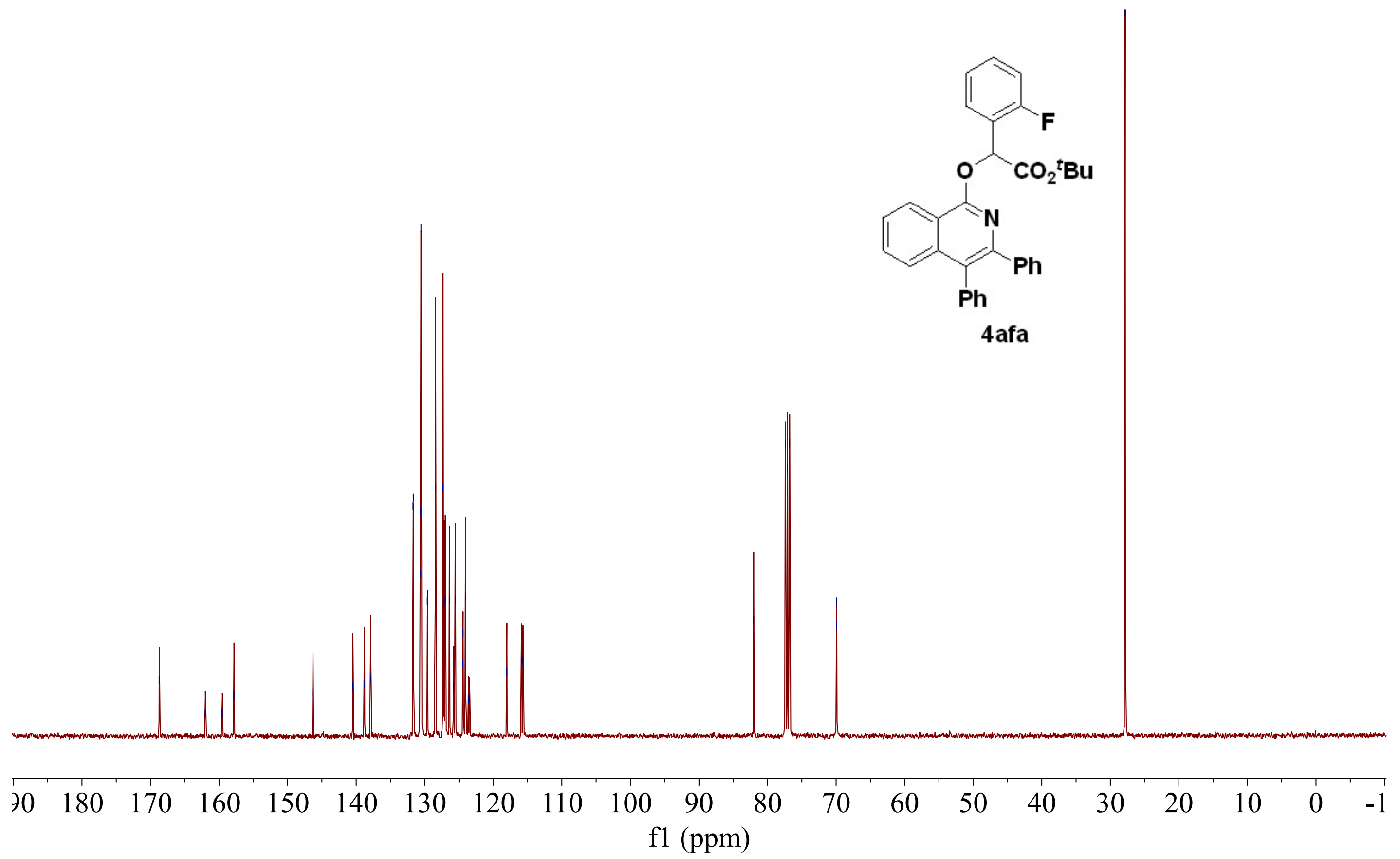




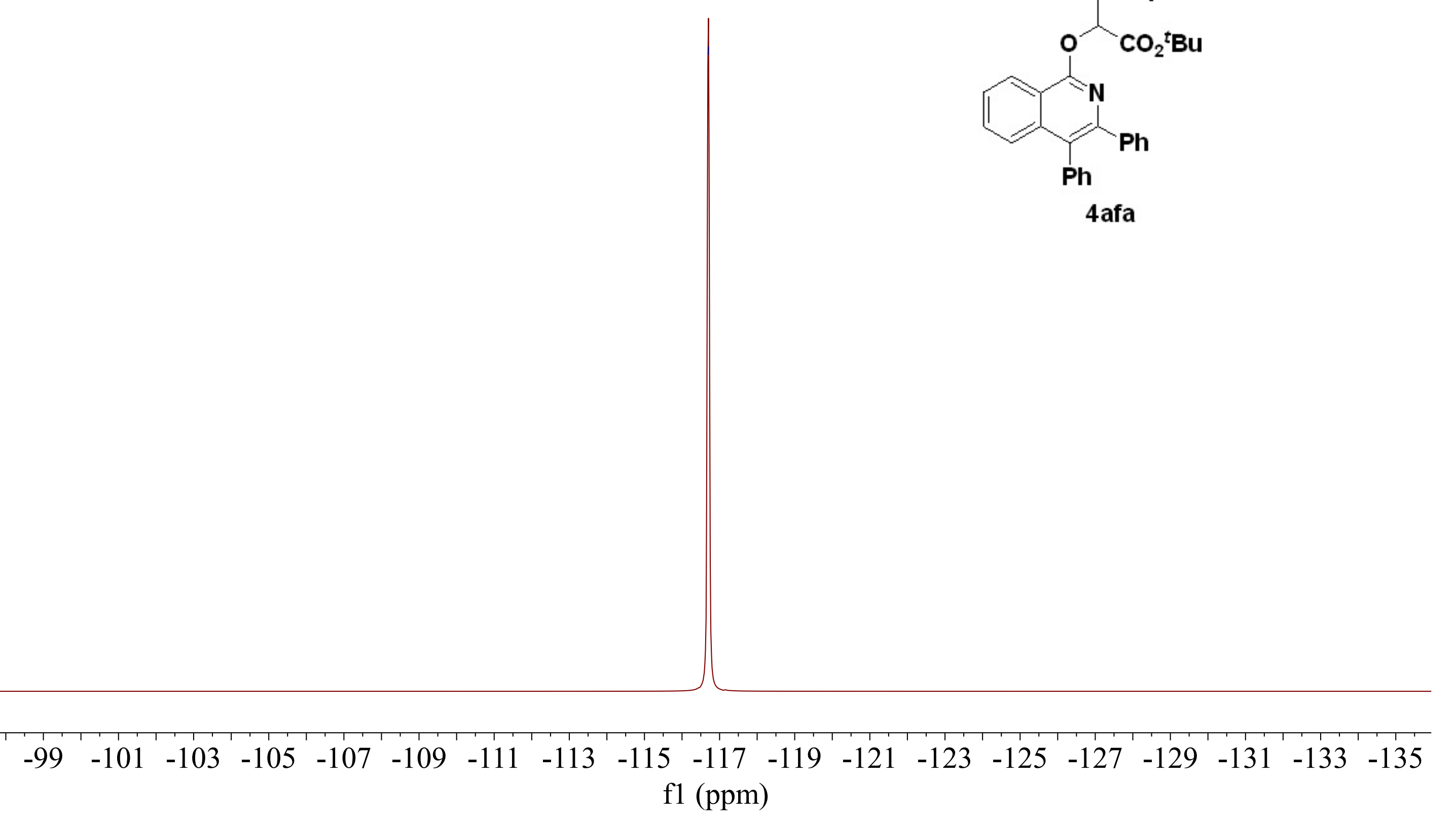




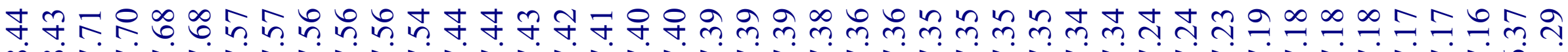

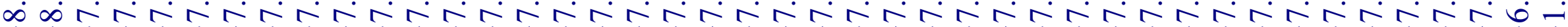

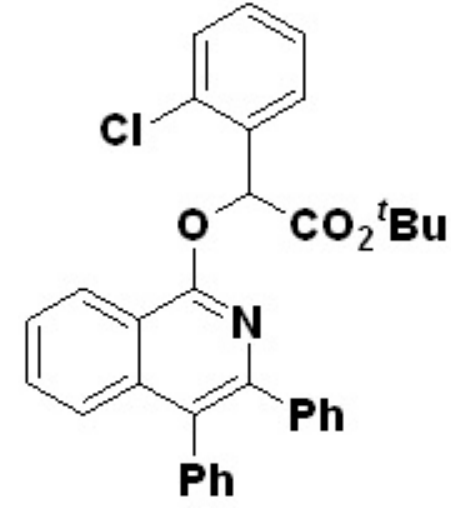

4aga

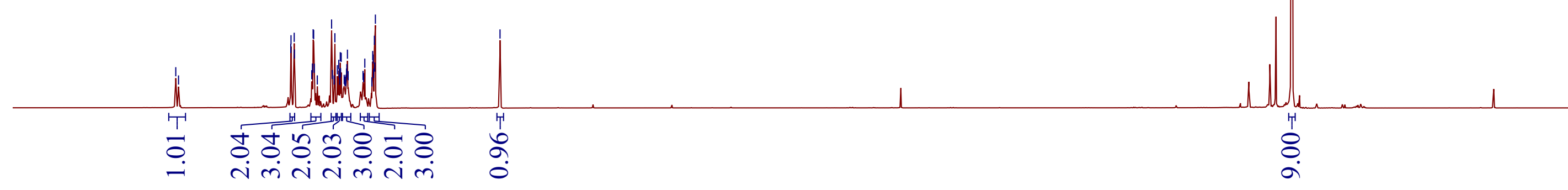

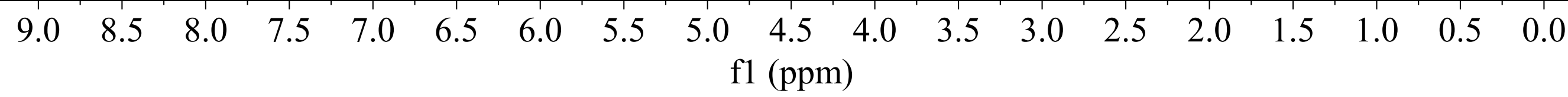



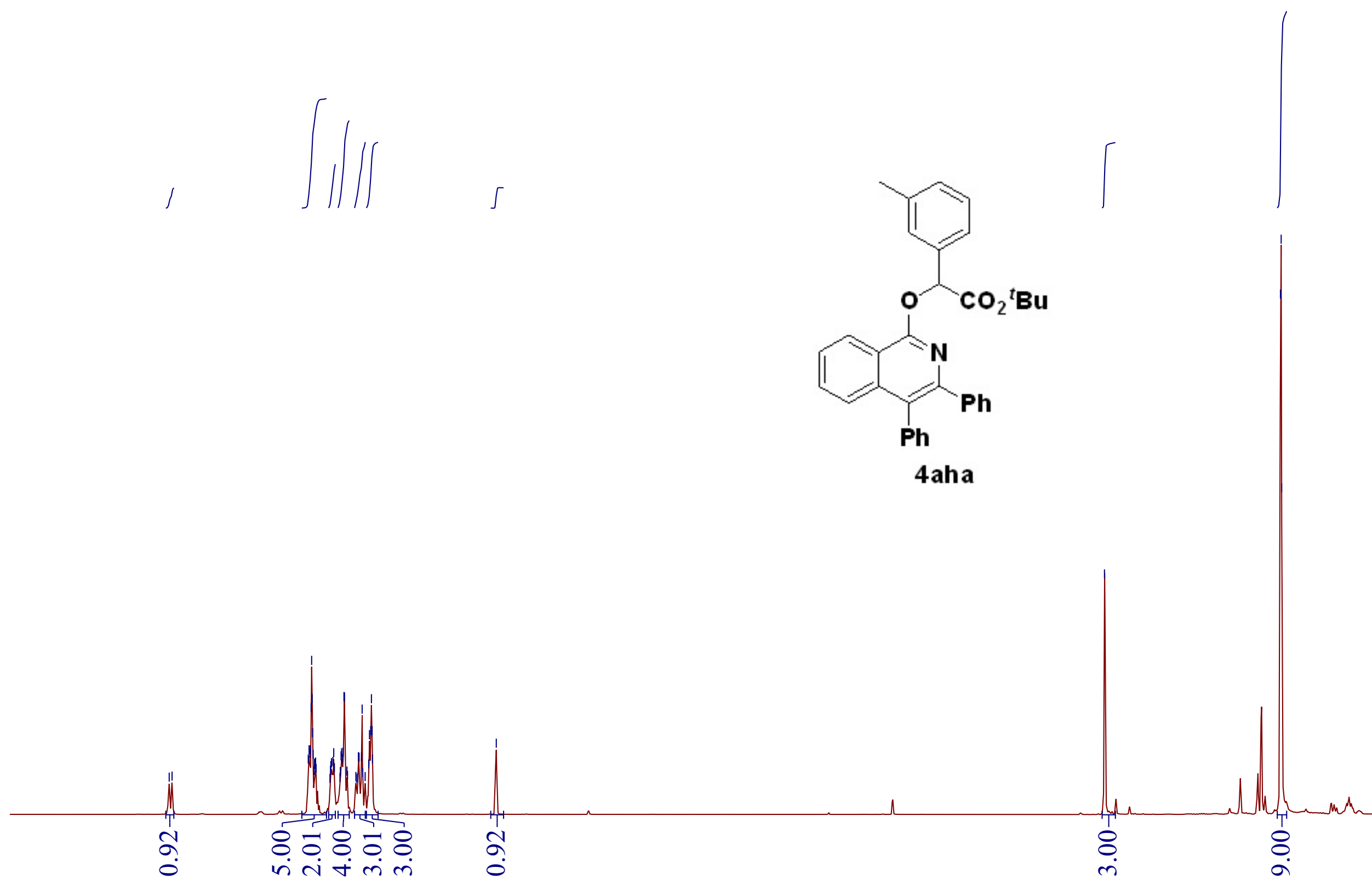

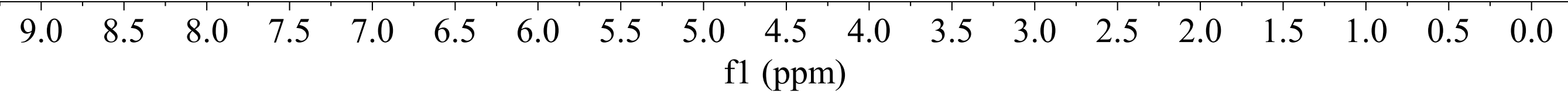



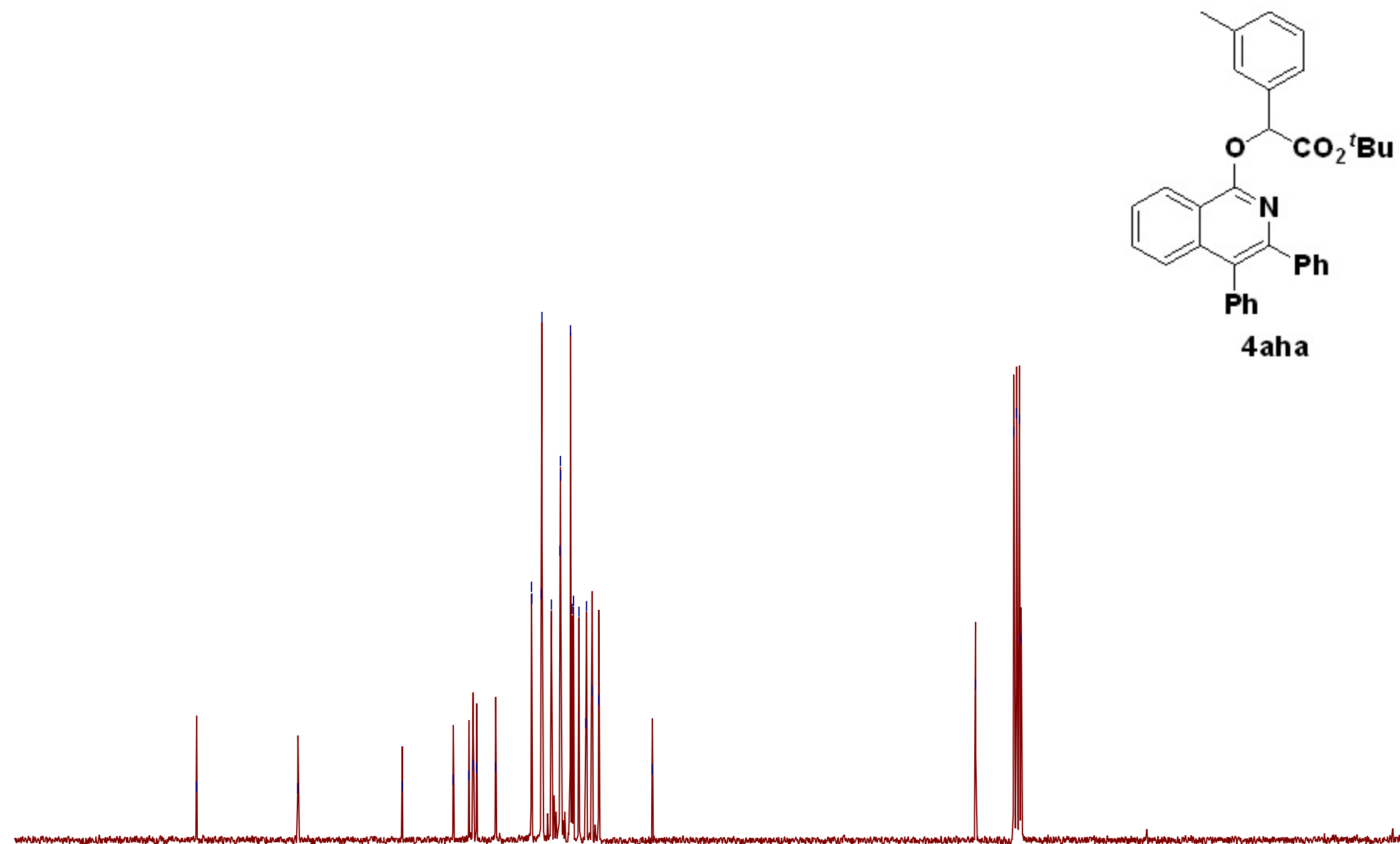

4aha 


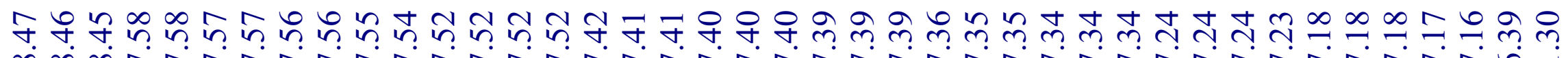

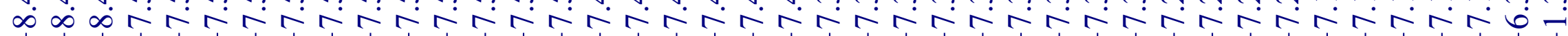
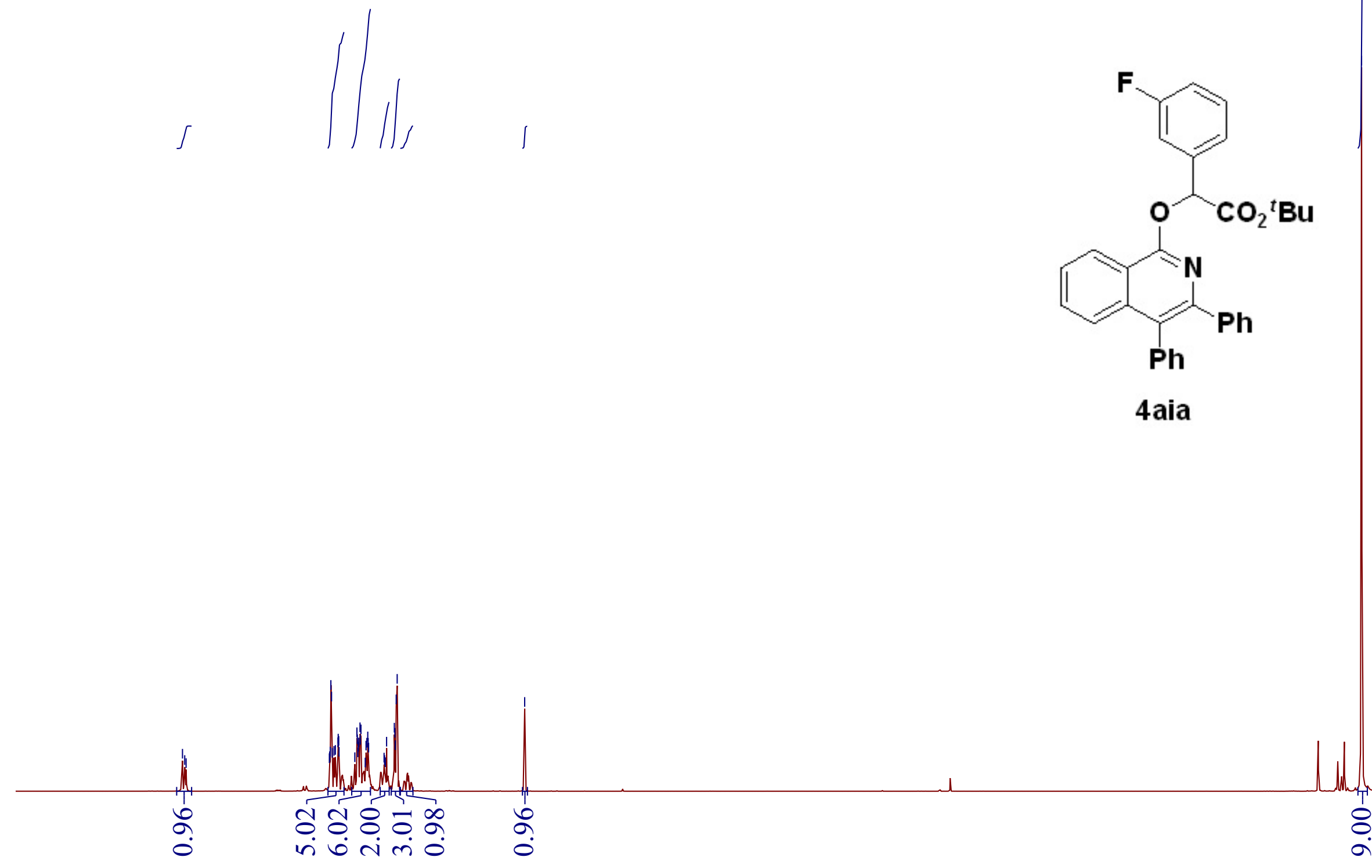

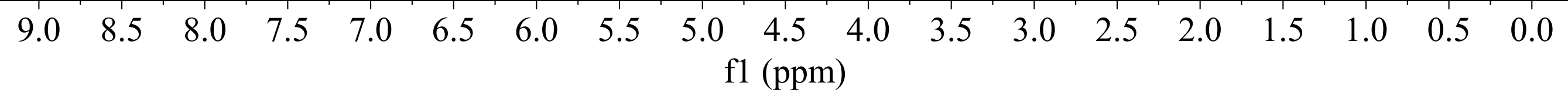


1イ イ

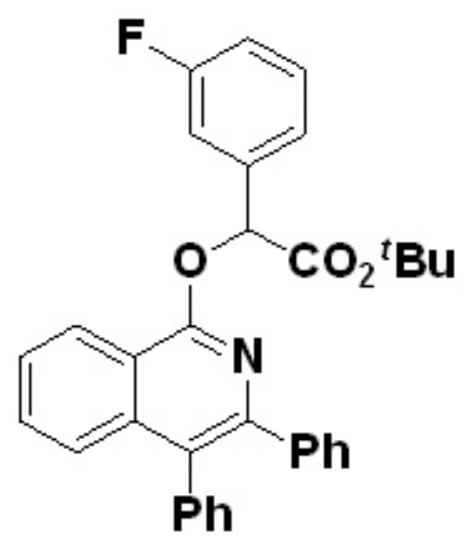

4aia $\begin{array}{rlllllllllll}0 & 180 & 170 & 160 & 150 & 140 & 130 & 120 & 110 & 100 & 90 & 80 \\ & & & & & \end{array}$ 
$\stackrel{0}{n}$

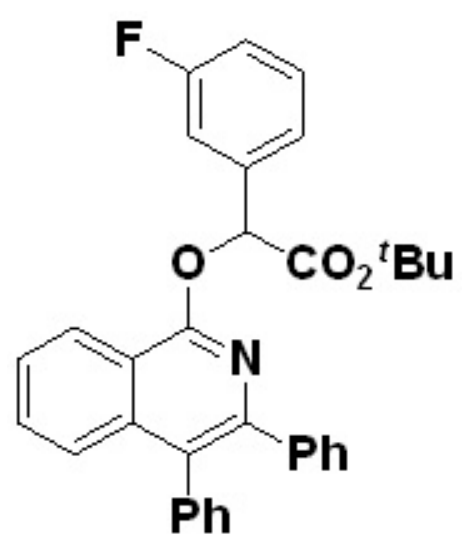

4aia

$\begin{array}{ccccccccccccccccccccccccccccc}100 & 80 & 60 & 40 & 20 & 0 & -20 & -40 & -60 & -80 & -100 & -120 & -140 & -160 & -180 & -200 & -220 & -240 & -260 & -280 & -300\end{array}$



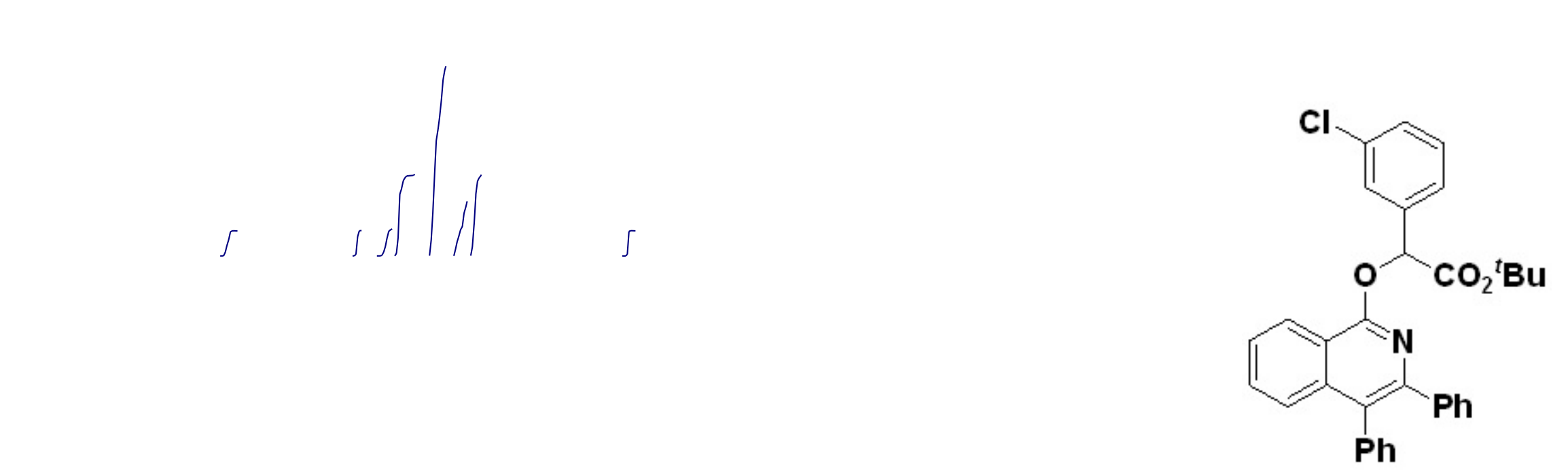

4aja

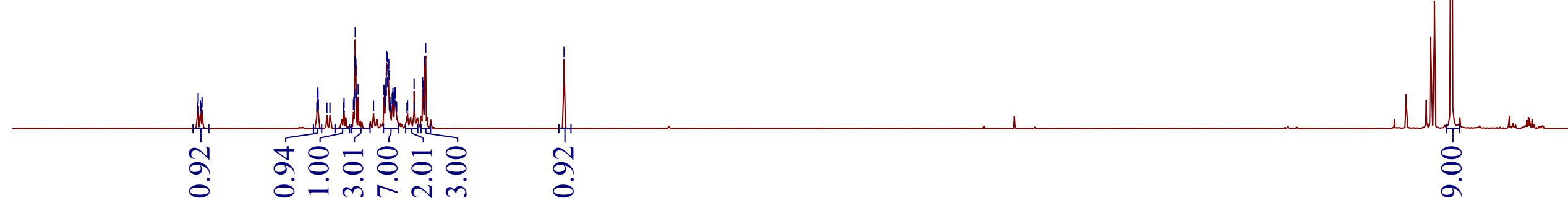

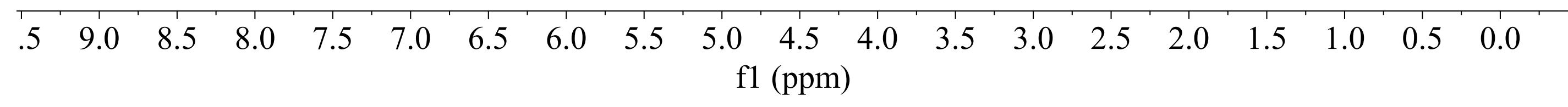




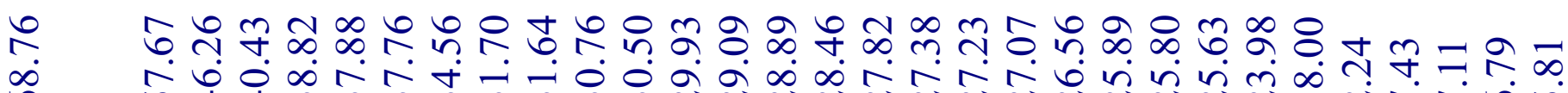

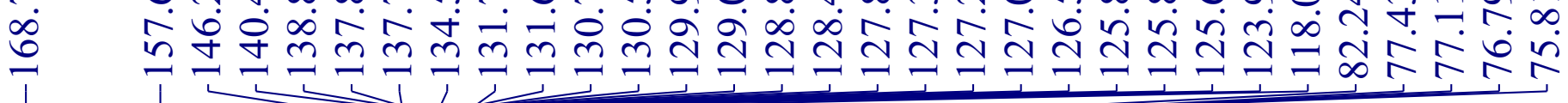

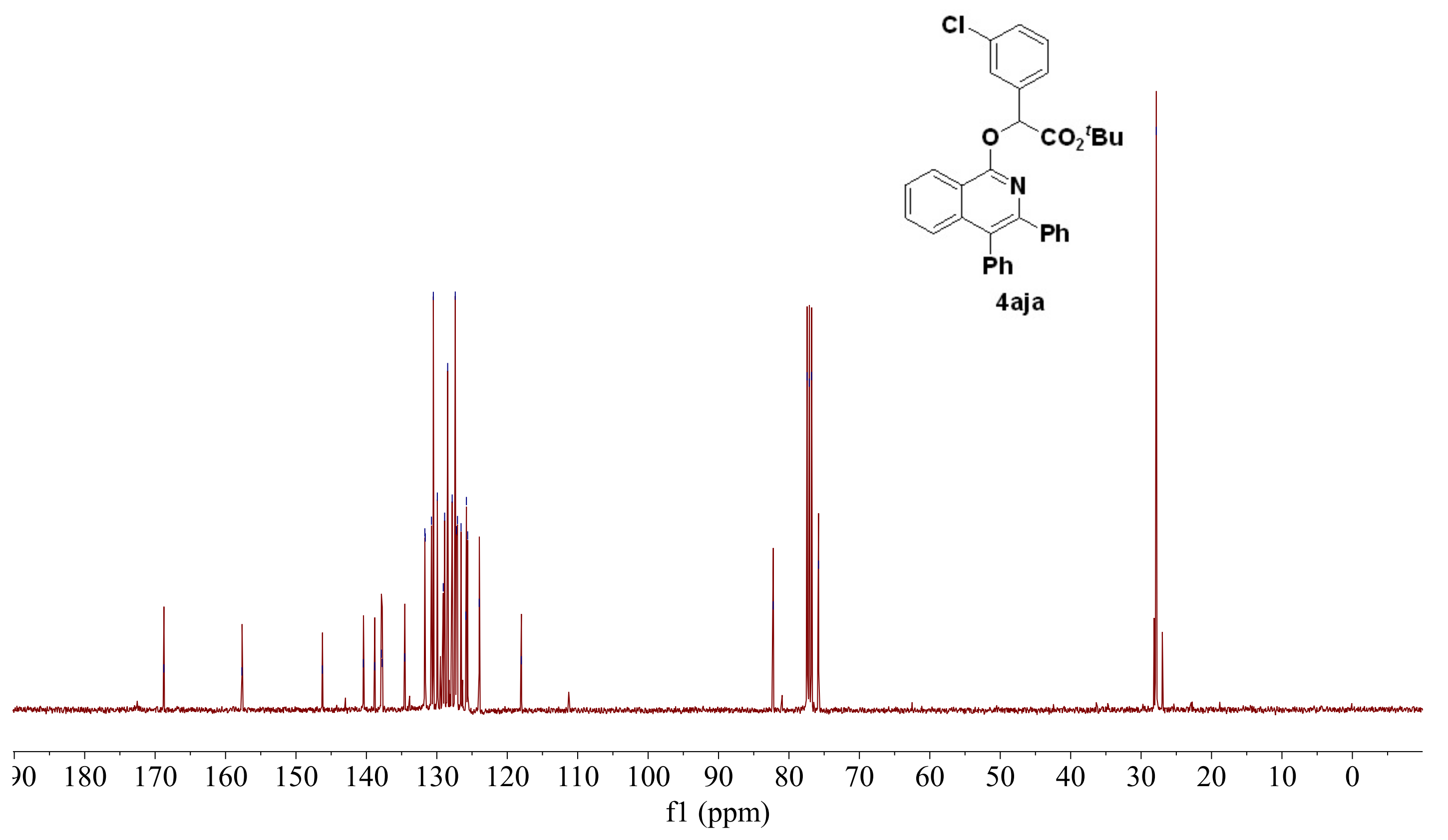




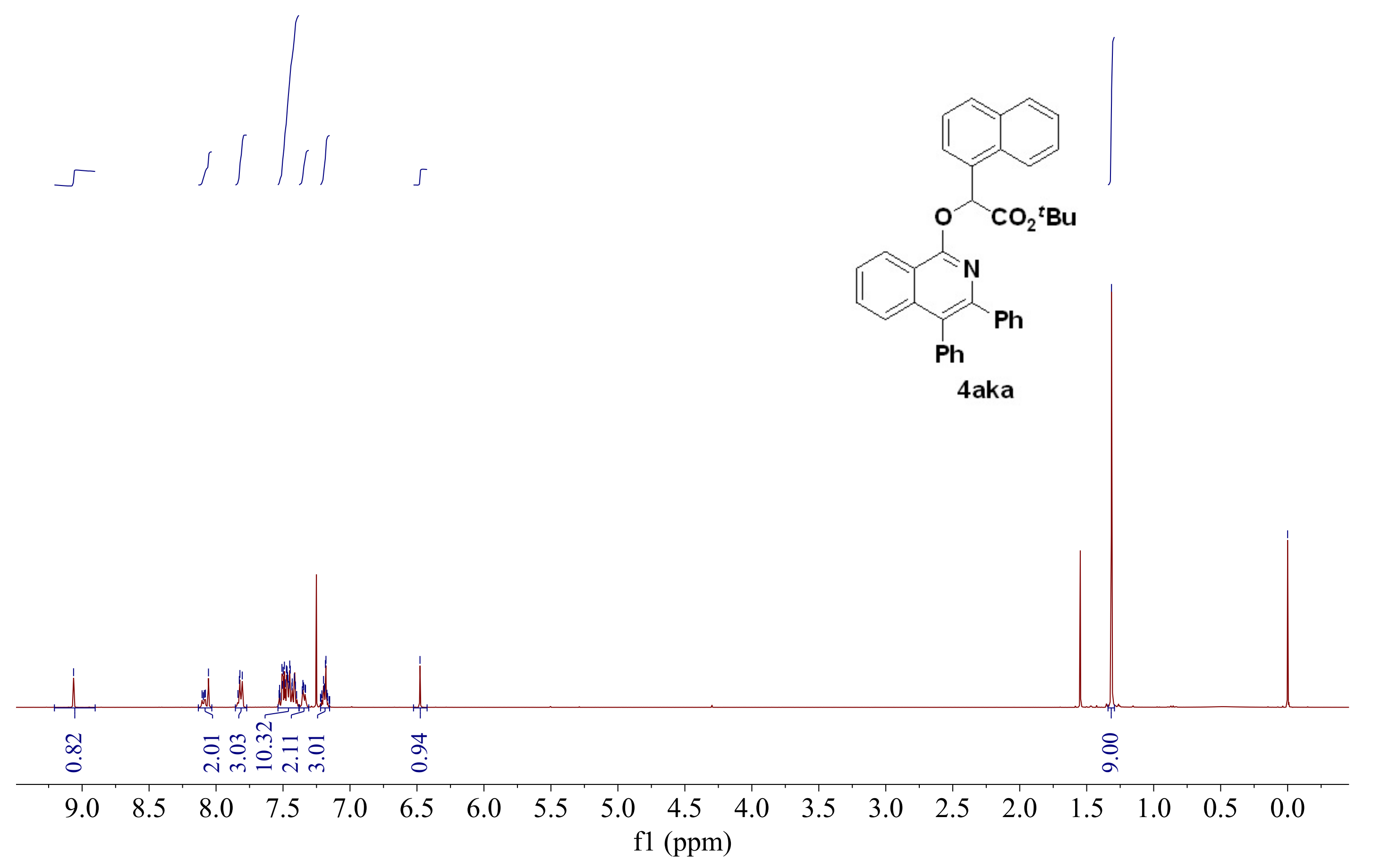




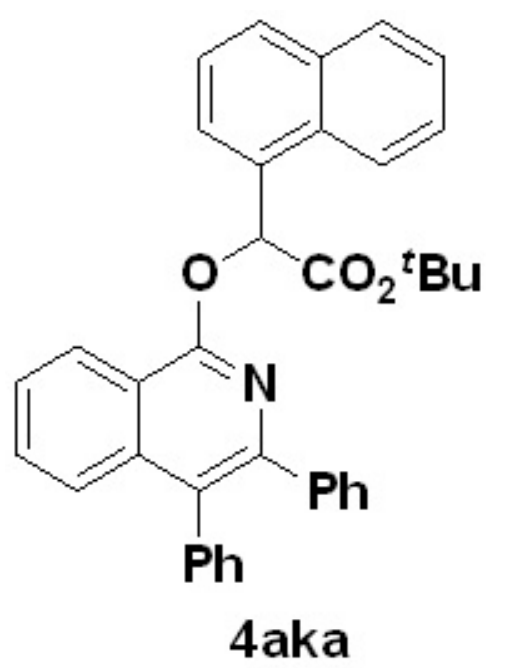

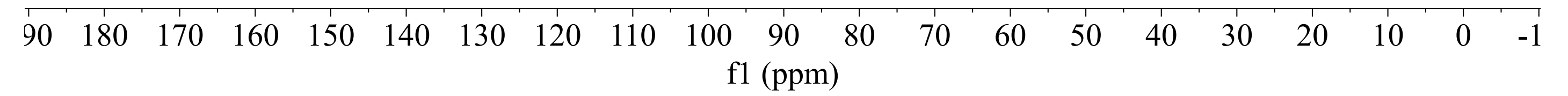




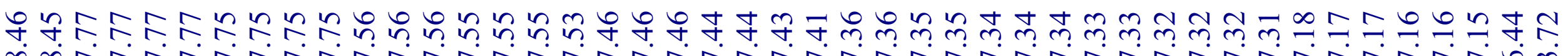

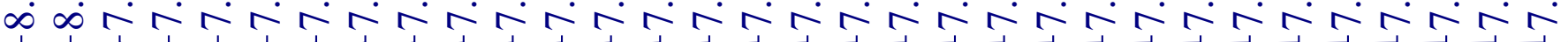
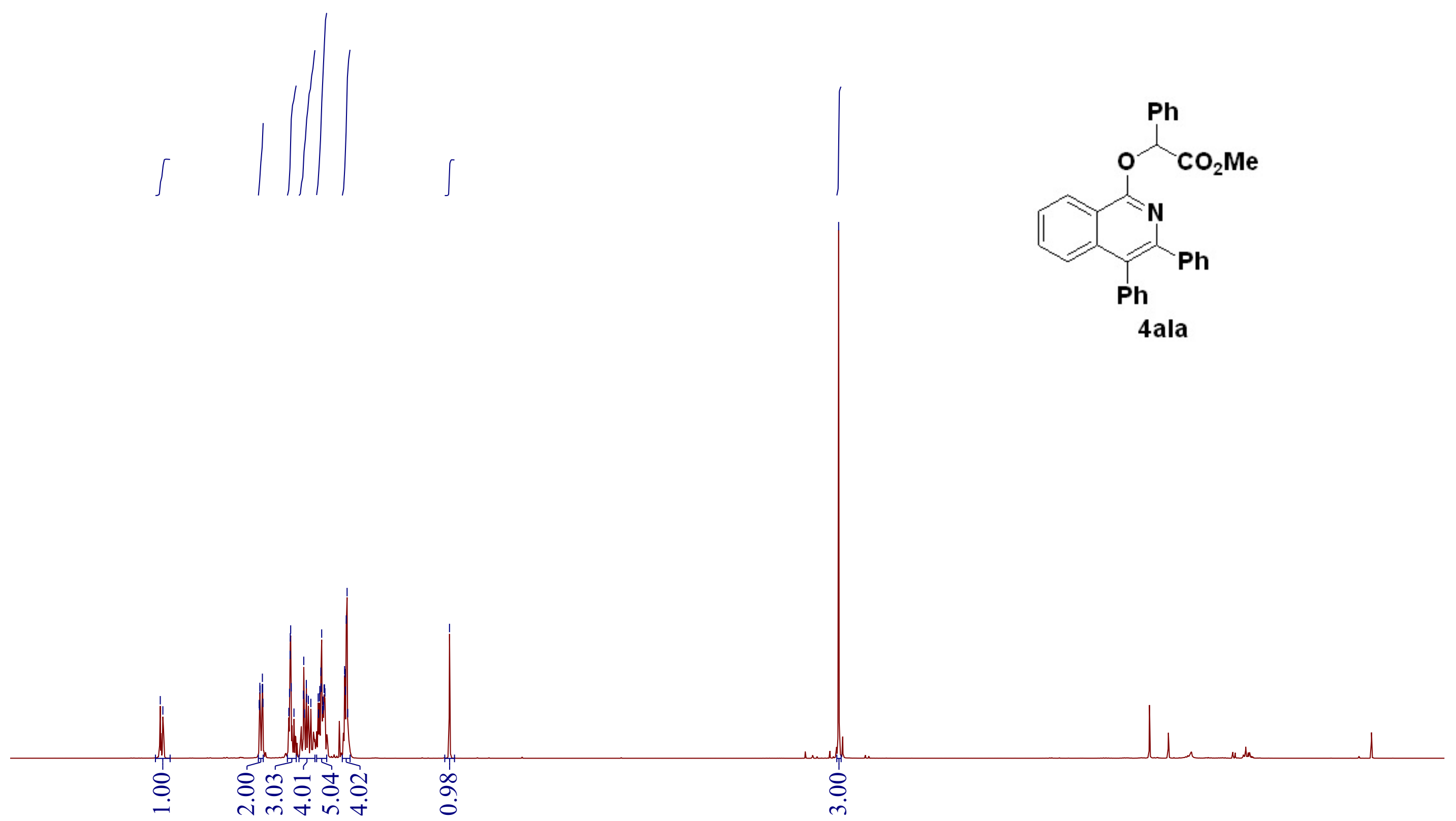

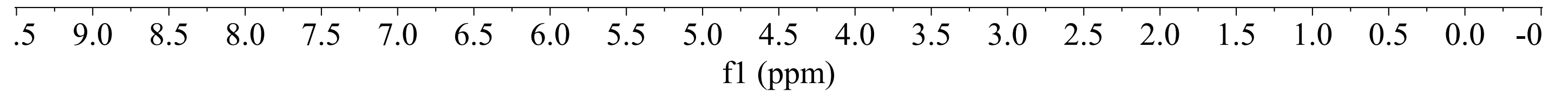




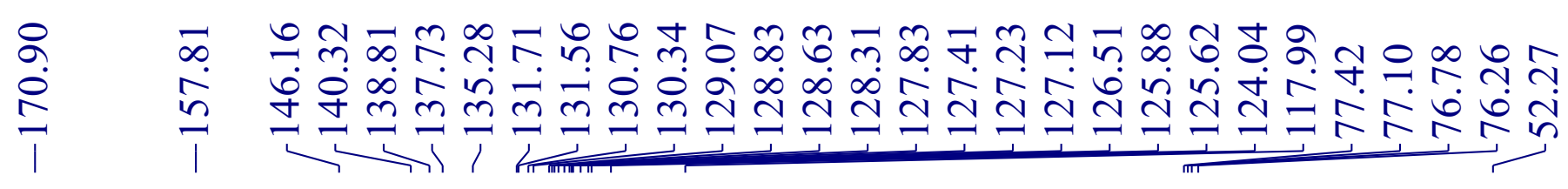
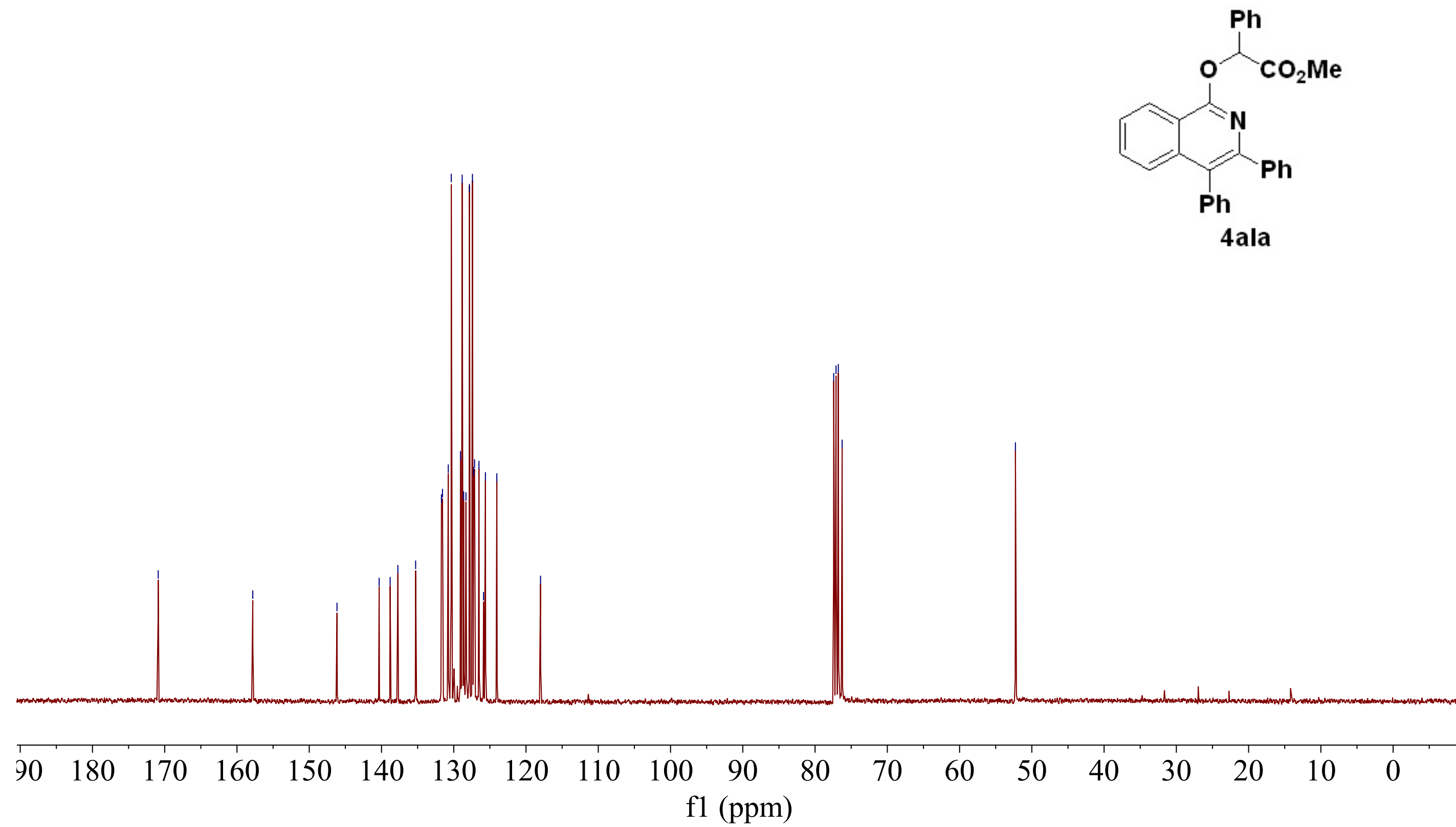


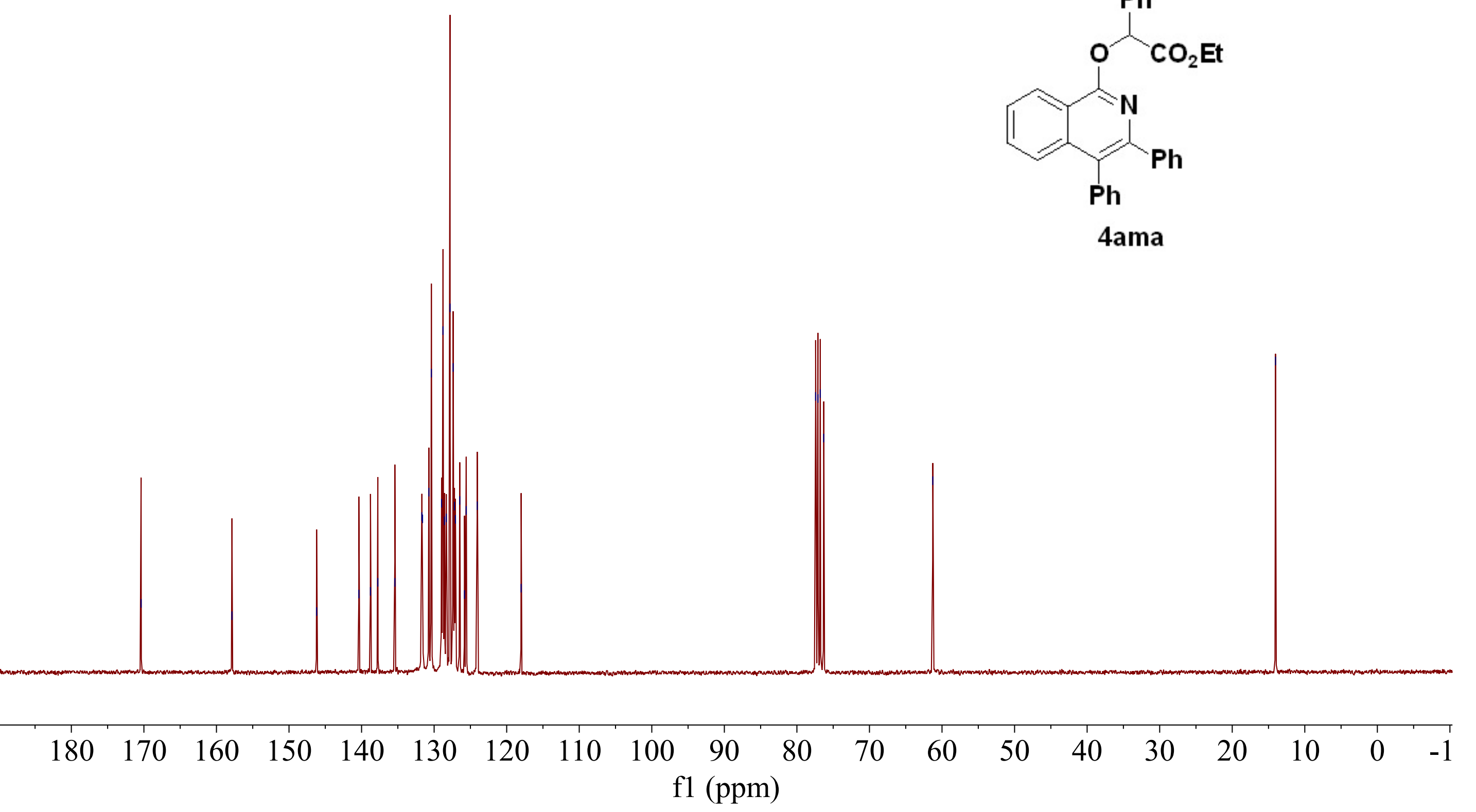




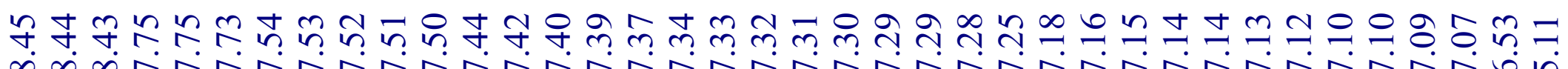
$\infty \infty_{\infty}$ NNNNNNNNNNNNNNNNNN
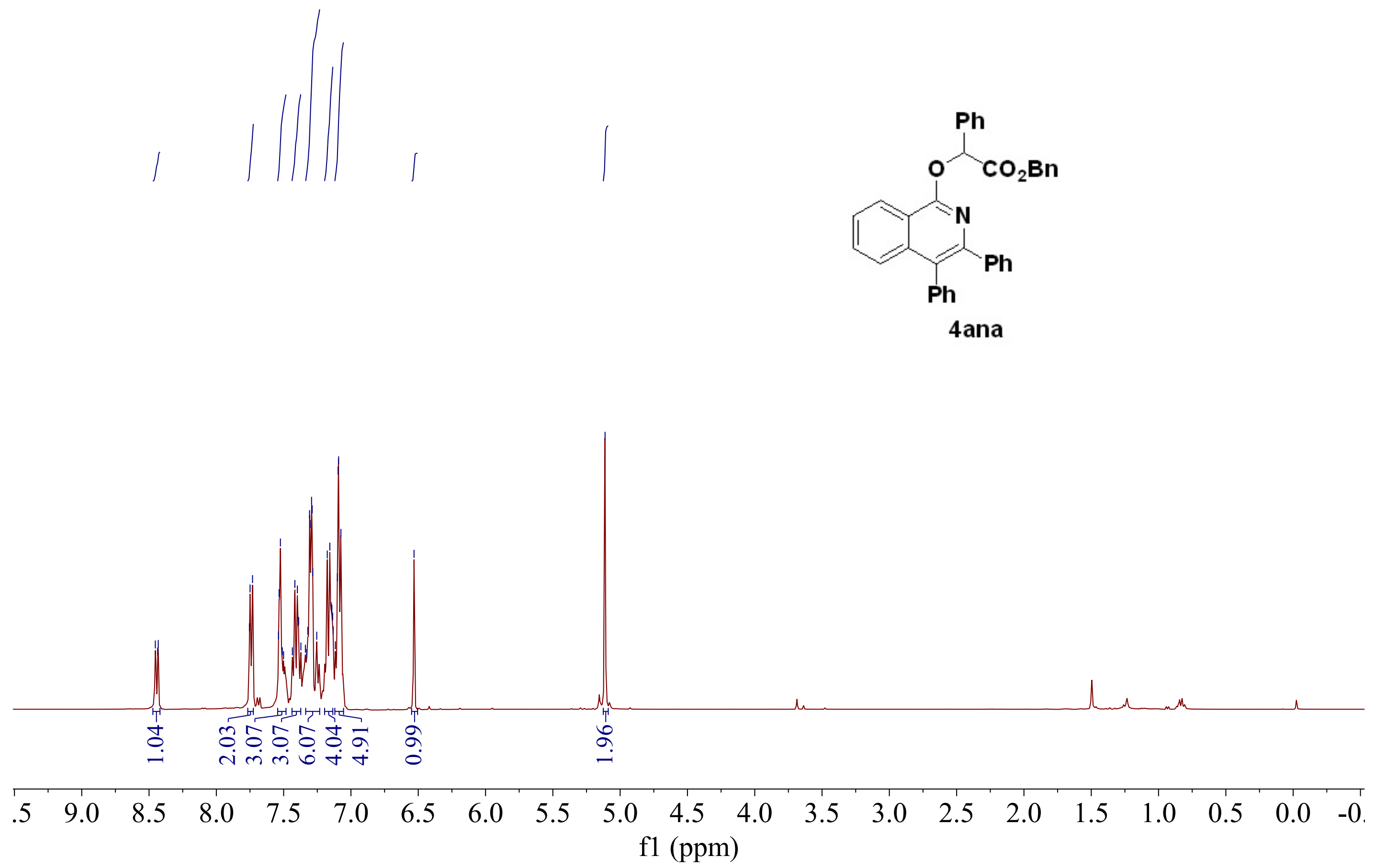


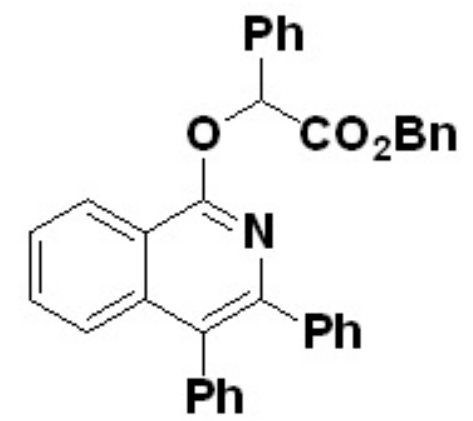

4ana

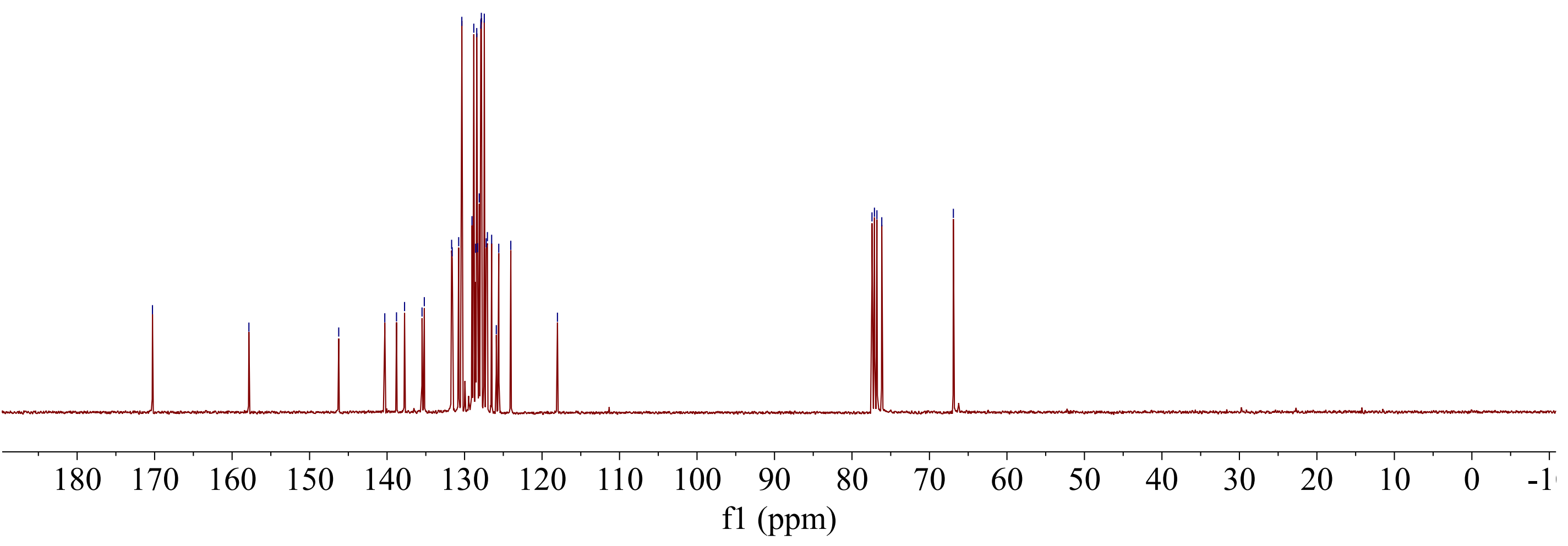



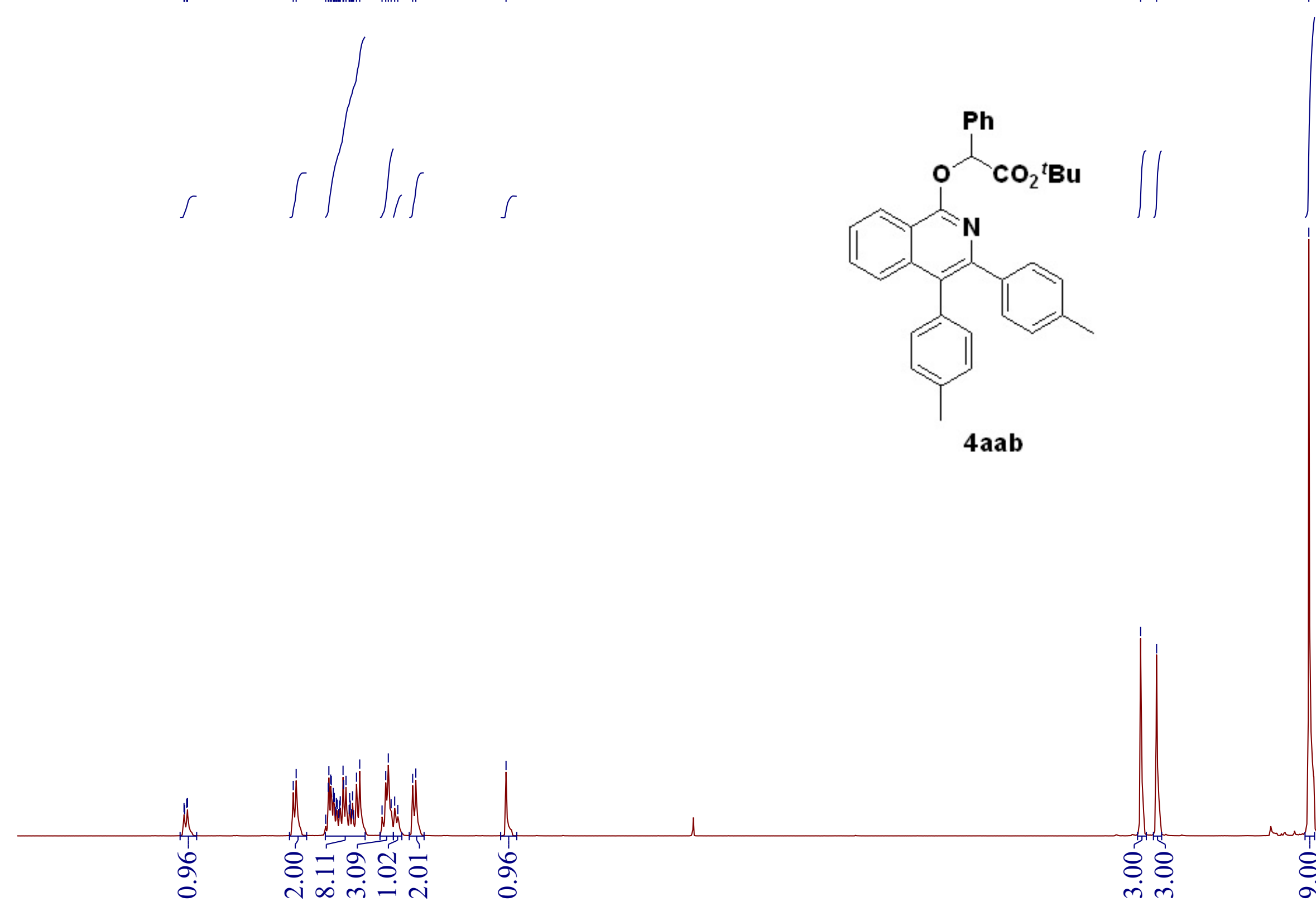

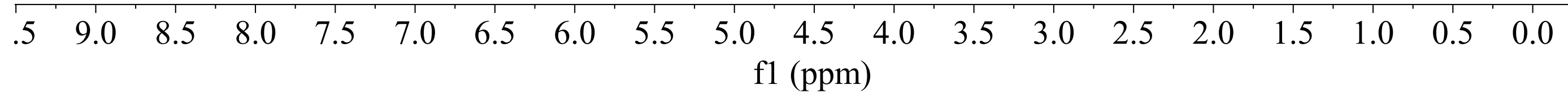




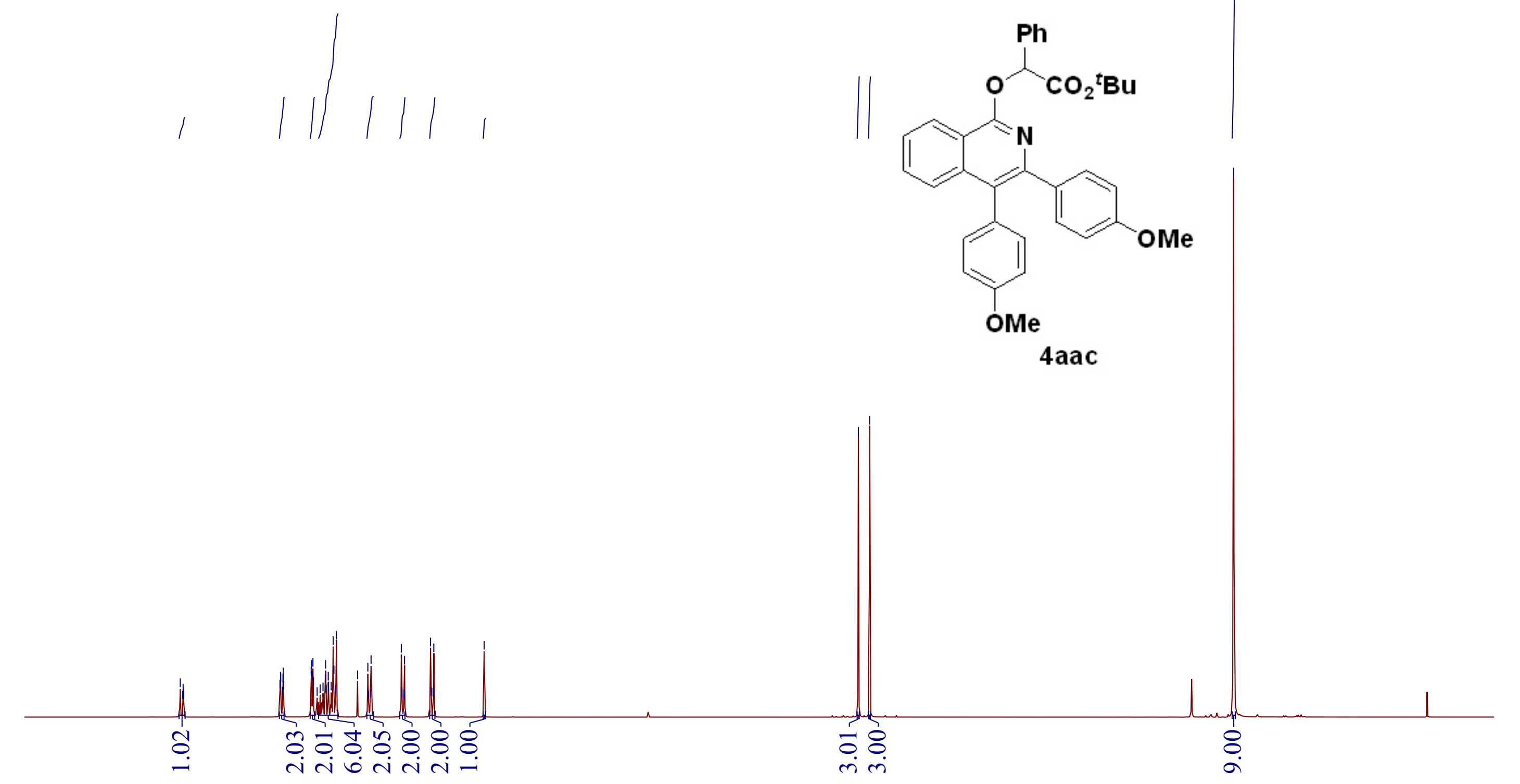

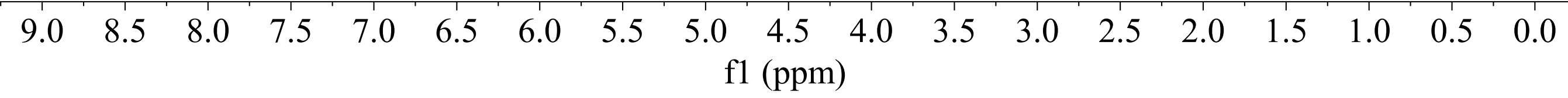




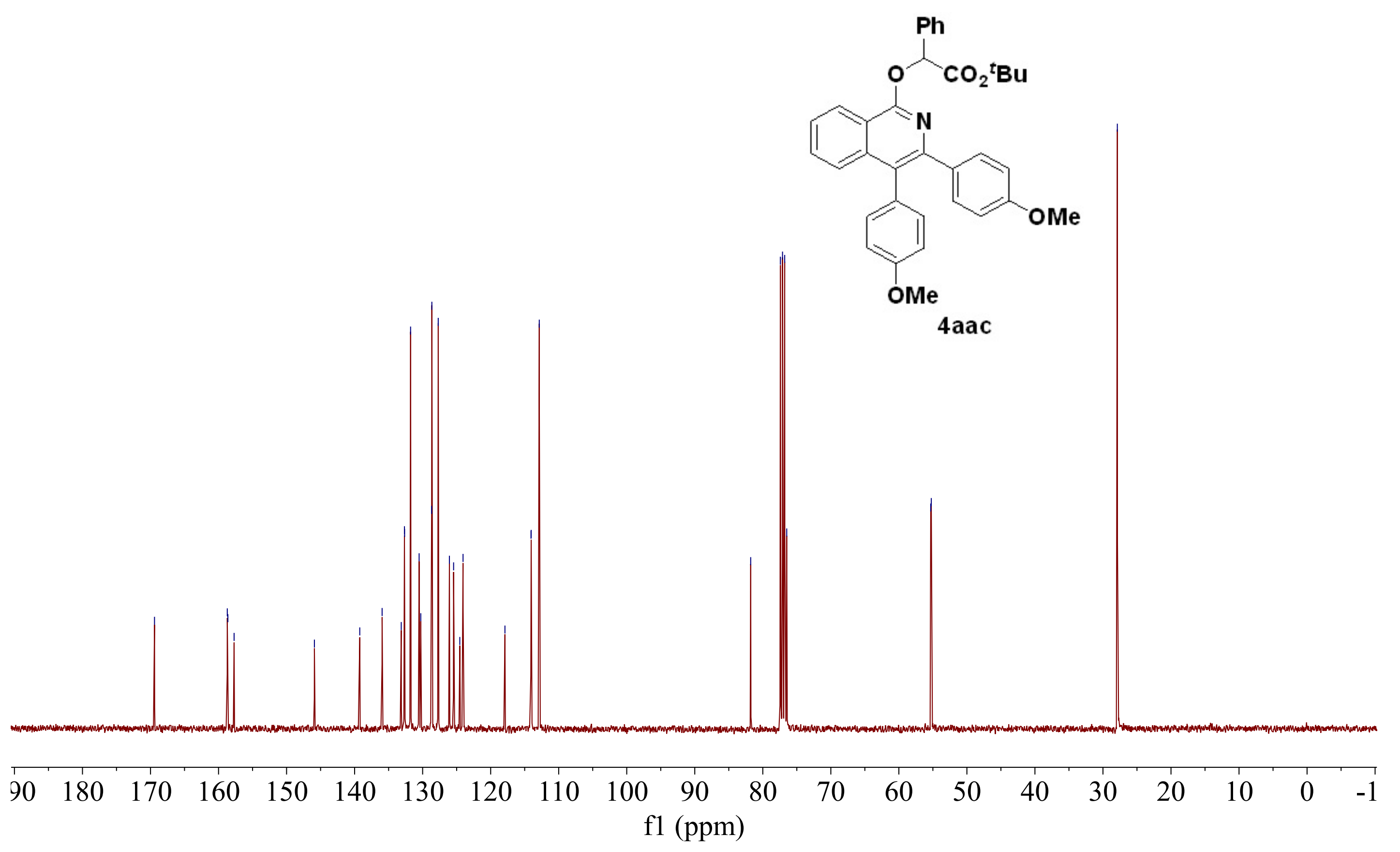




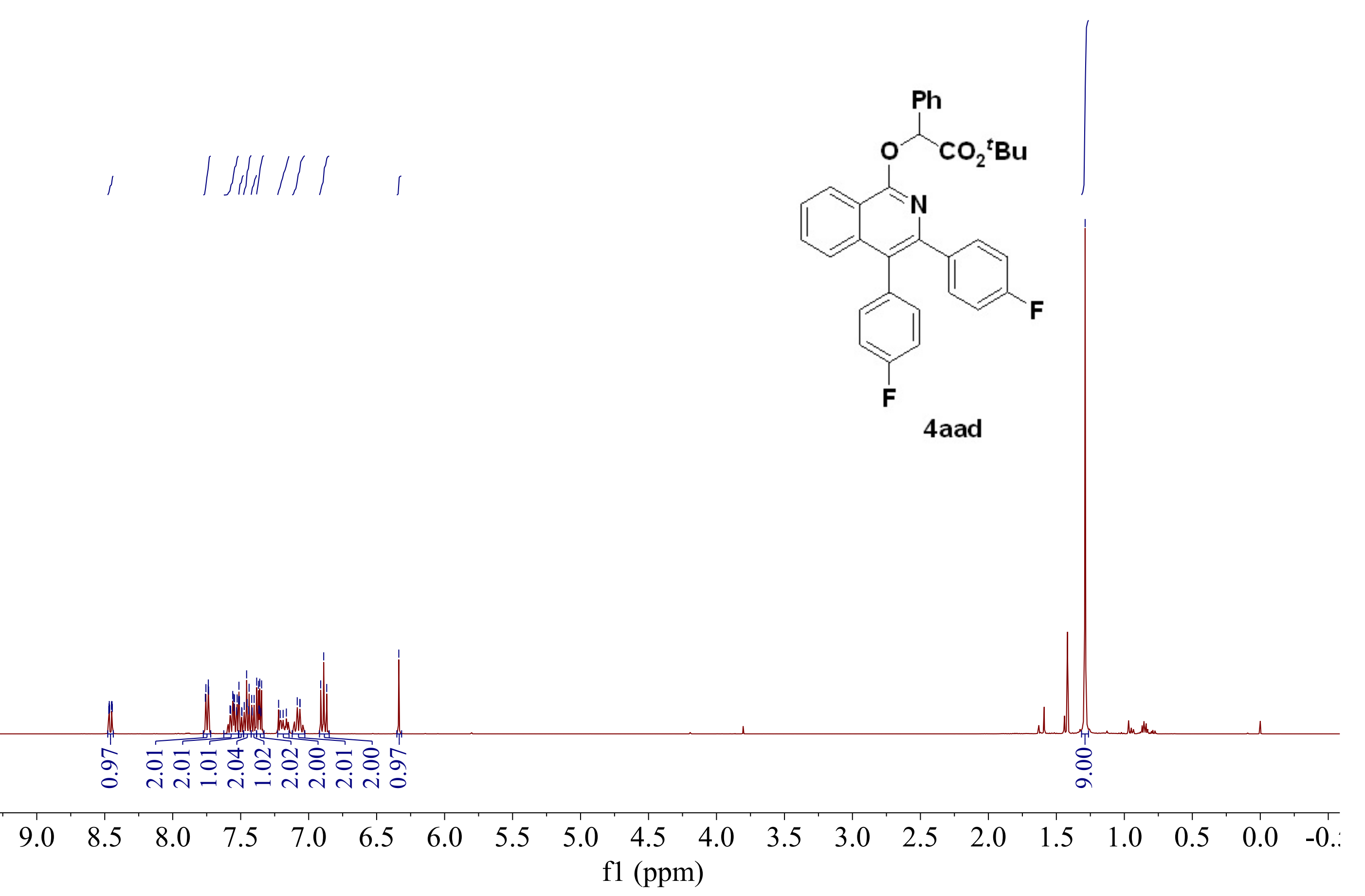




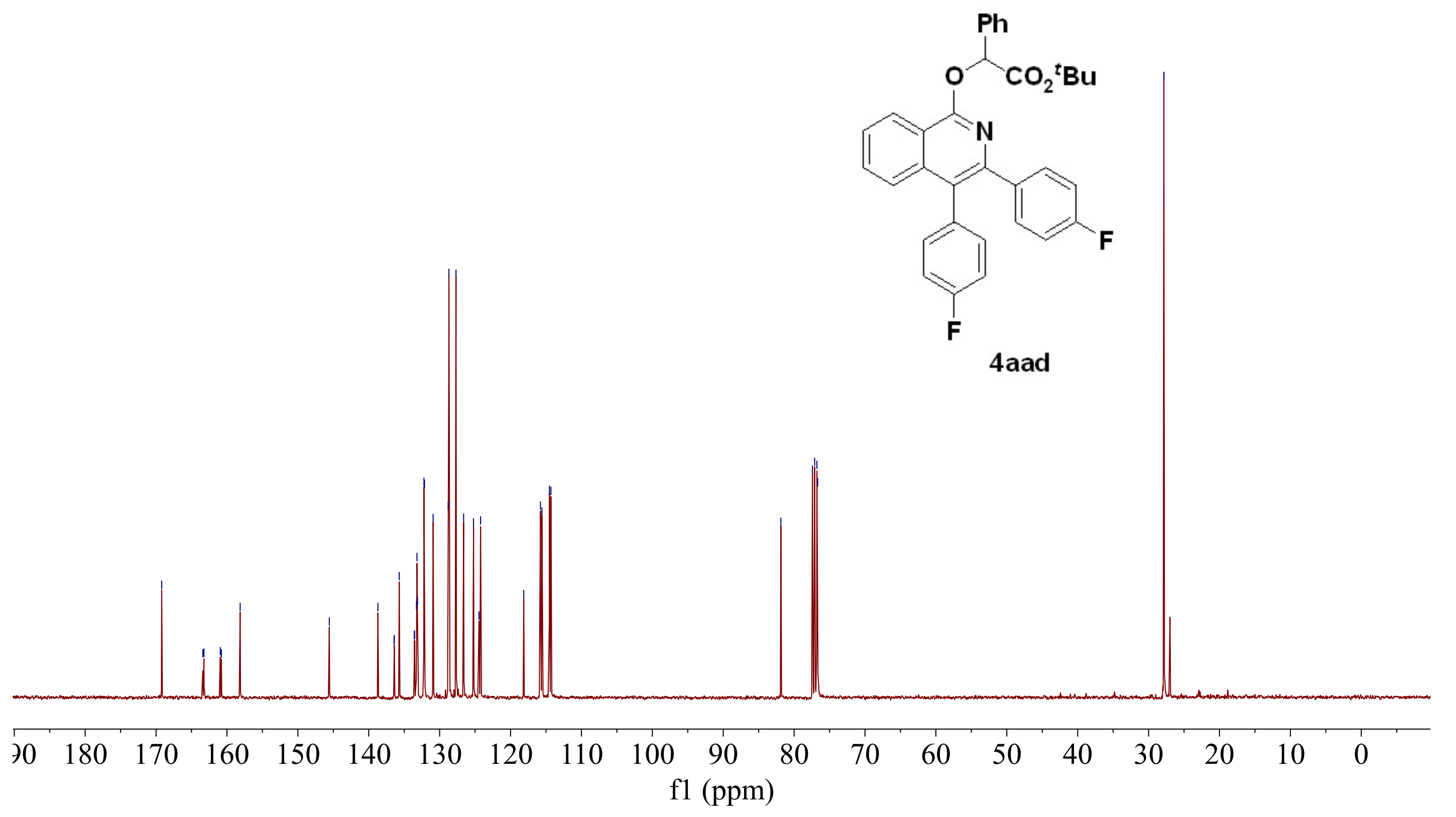




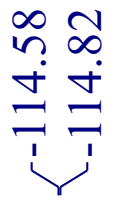

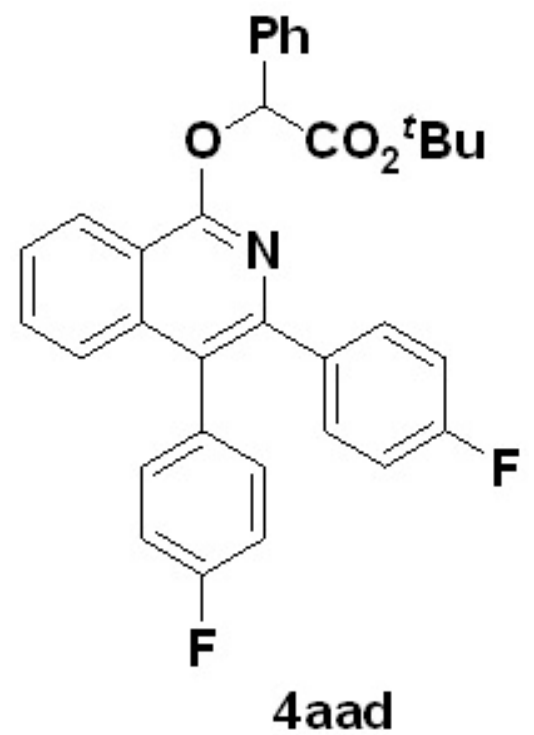

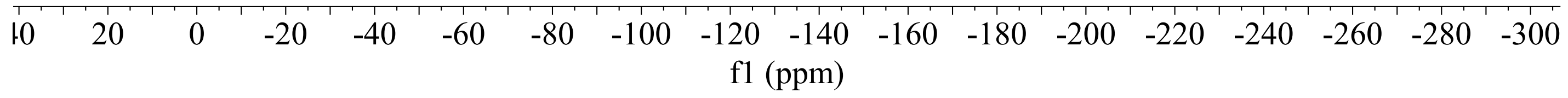


ๆ Lo
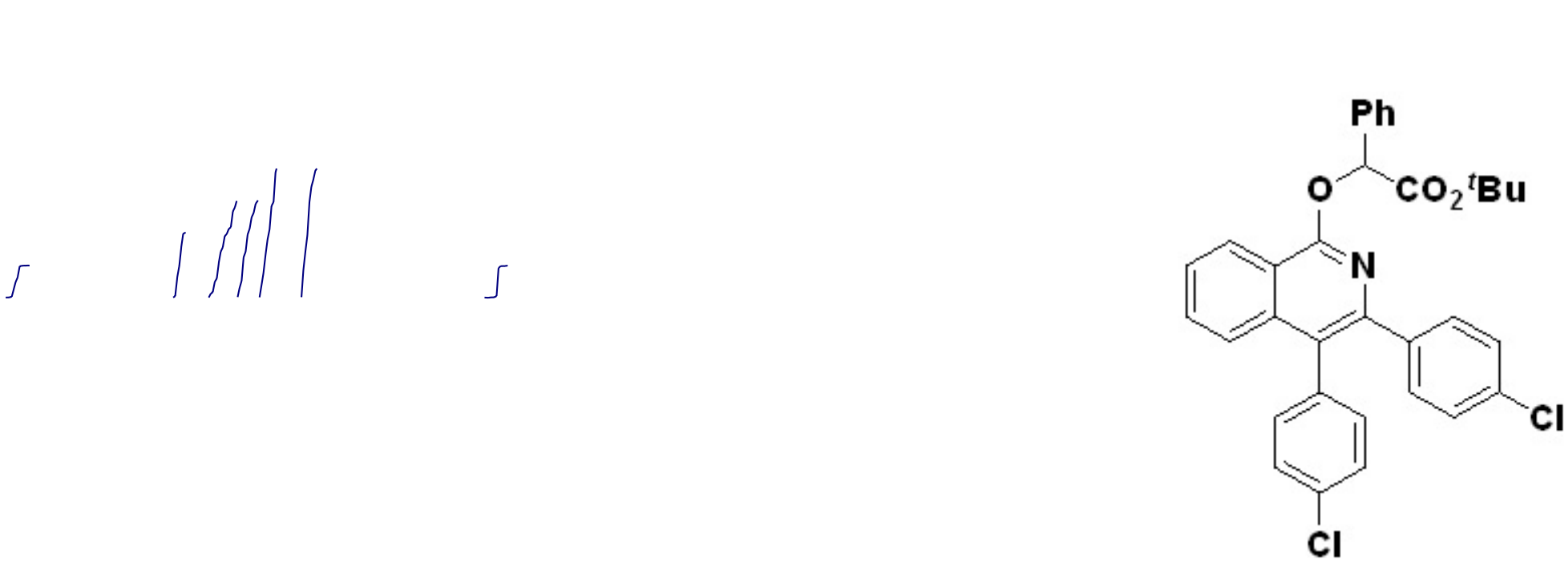

4aae

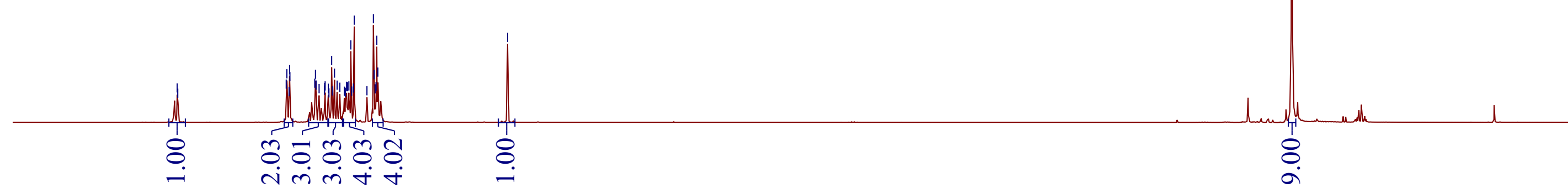

\begin{tabular}{llllllllllllllllllll}
\hline .5 & 9.0 & 8.5 & 8.0 & 7.5 & 7.0 & 6.5 & 6.0 & 5.5 & $\begin{array}{c}5.0 \\
\text { f1 }(\mathrm{ppm})\end{array}$ & $\begin{array}{c}4.5 \\
4.0\end{array}$ & 3.5 & 3.0 & 2.5 & 2.0 & 1.5 & 1.0 & 0.5 & 0.0
\end{tabular}




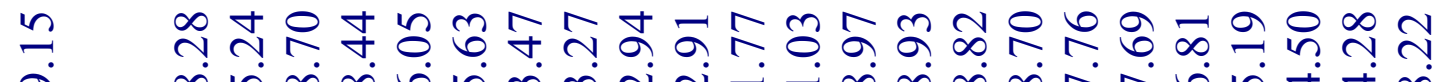

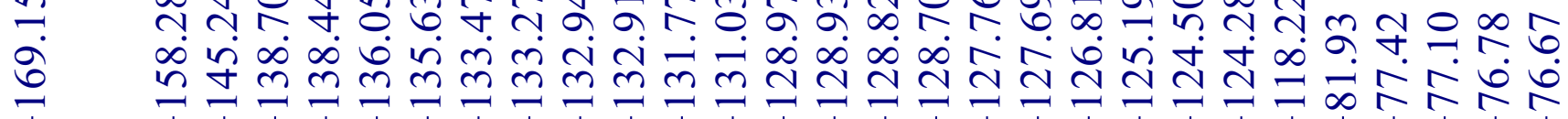

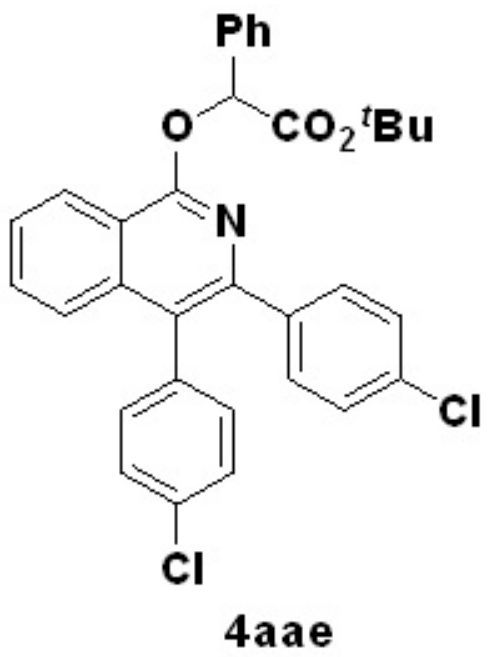

$$
\begin{array}{llllllllllllllllll}
180 & 170 & 160 & 150 & 140 & 130 & 120 & 110 & \begin{array}{c}
100 \\
\mathrm{f} 1
\end{array}\left(\begin{array}{c}
90 \\
(\mathrm{ppm})
\end{array}\right. & 80 & 70 & 60 & 50 & 40 & 30 & 20 & 10 & 0
\end{array}
$$



Q

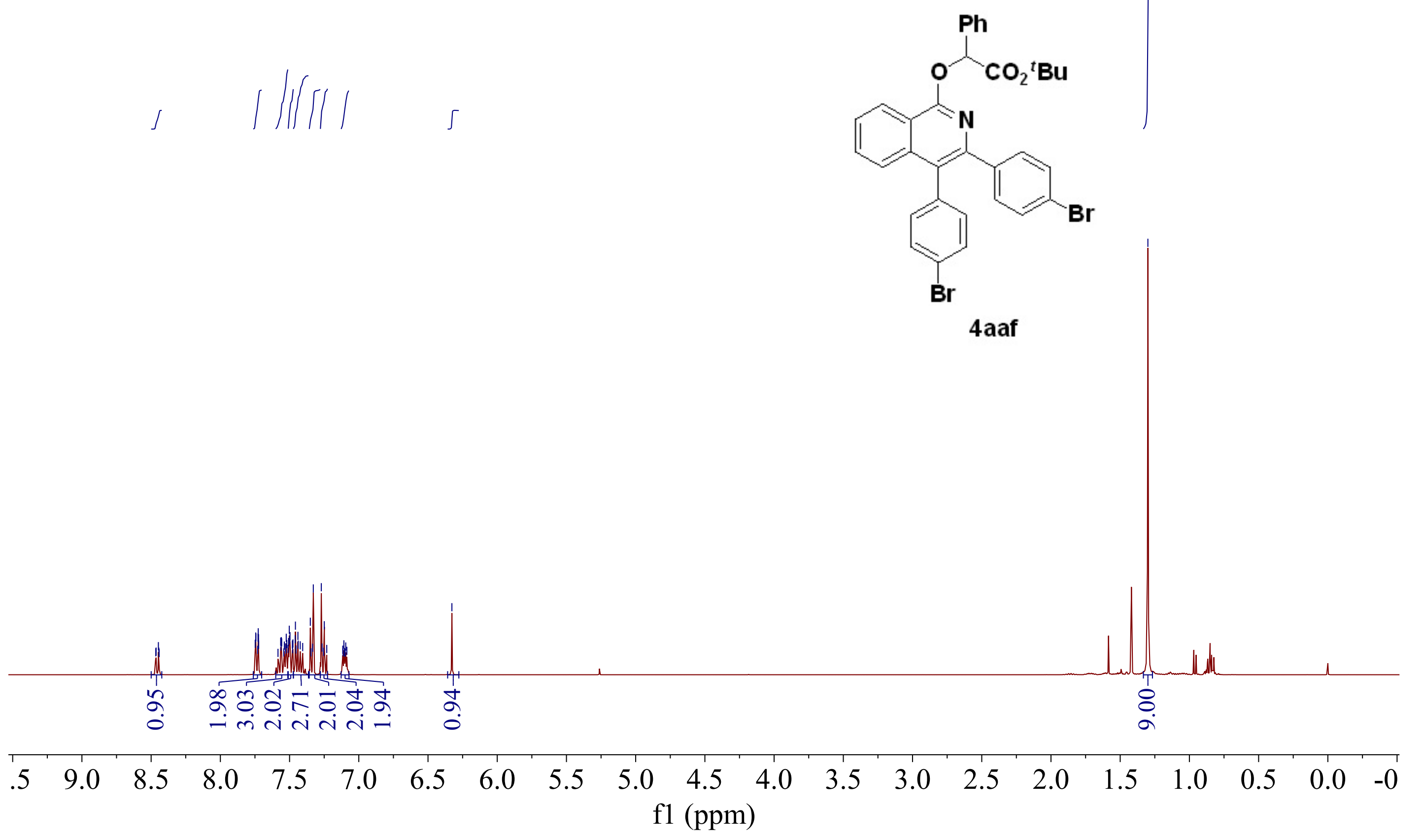




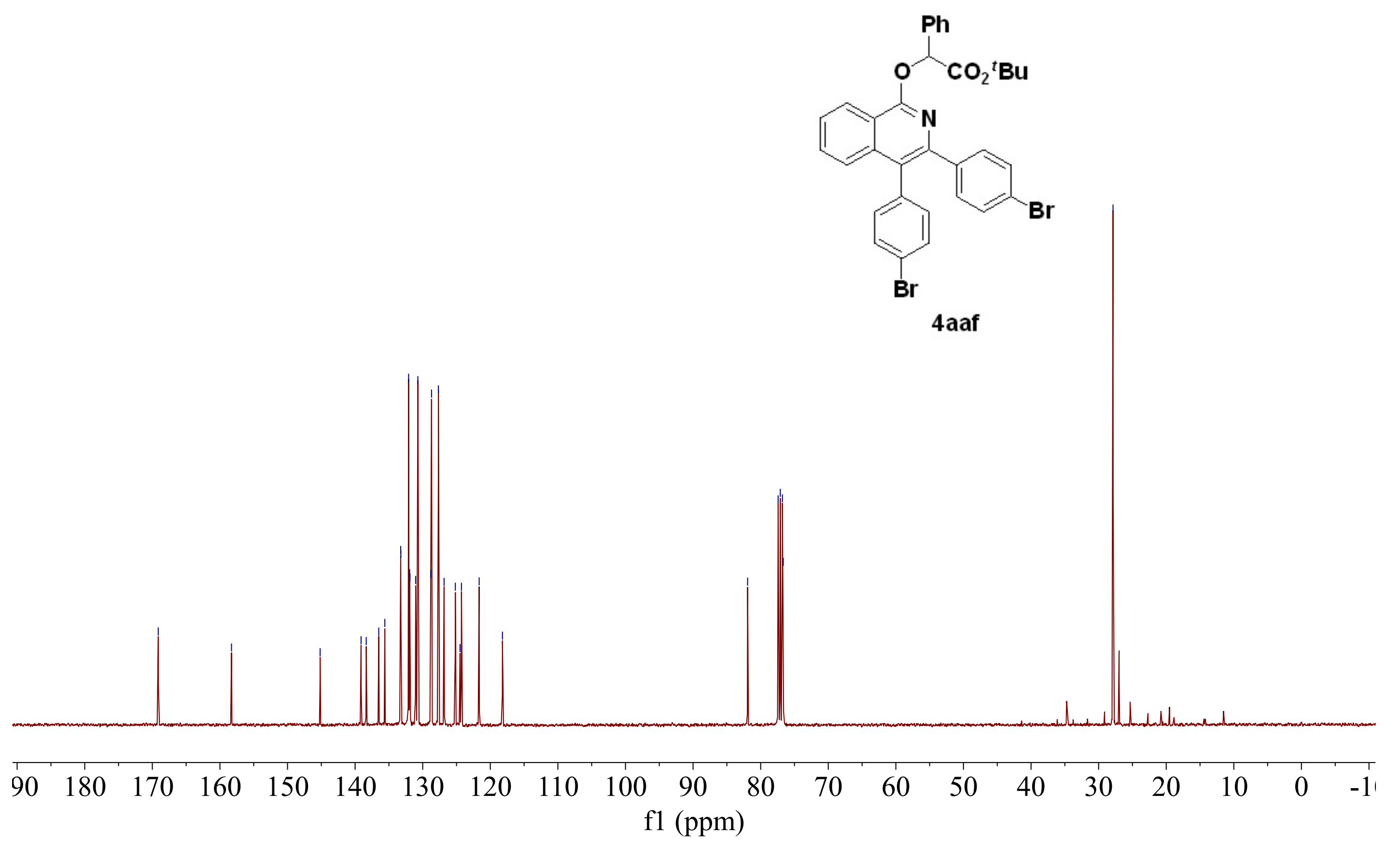




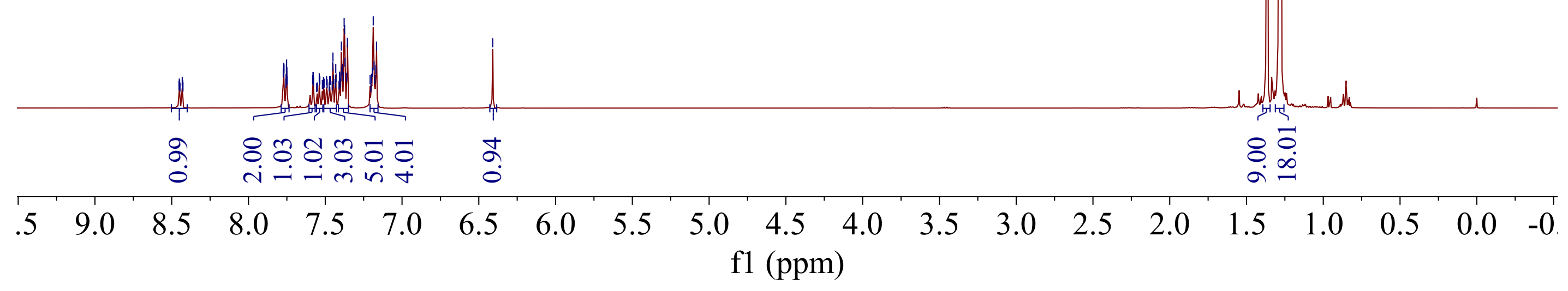




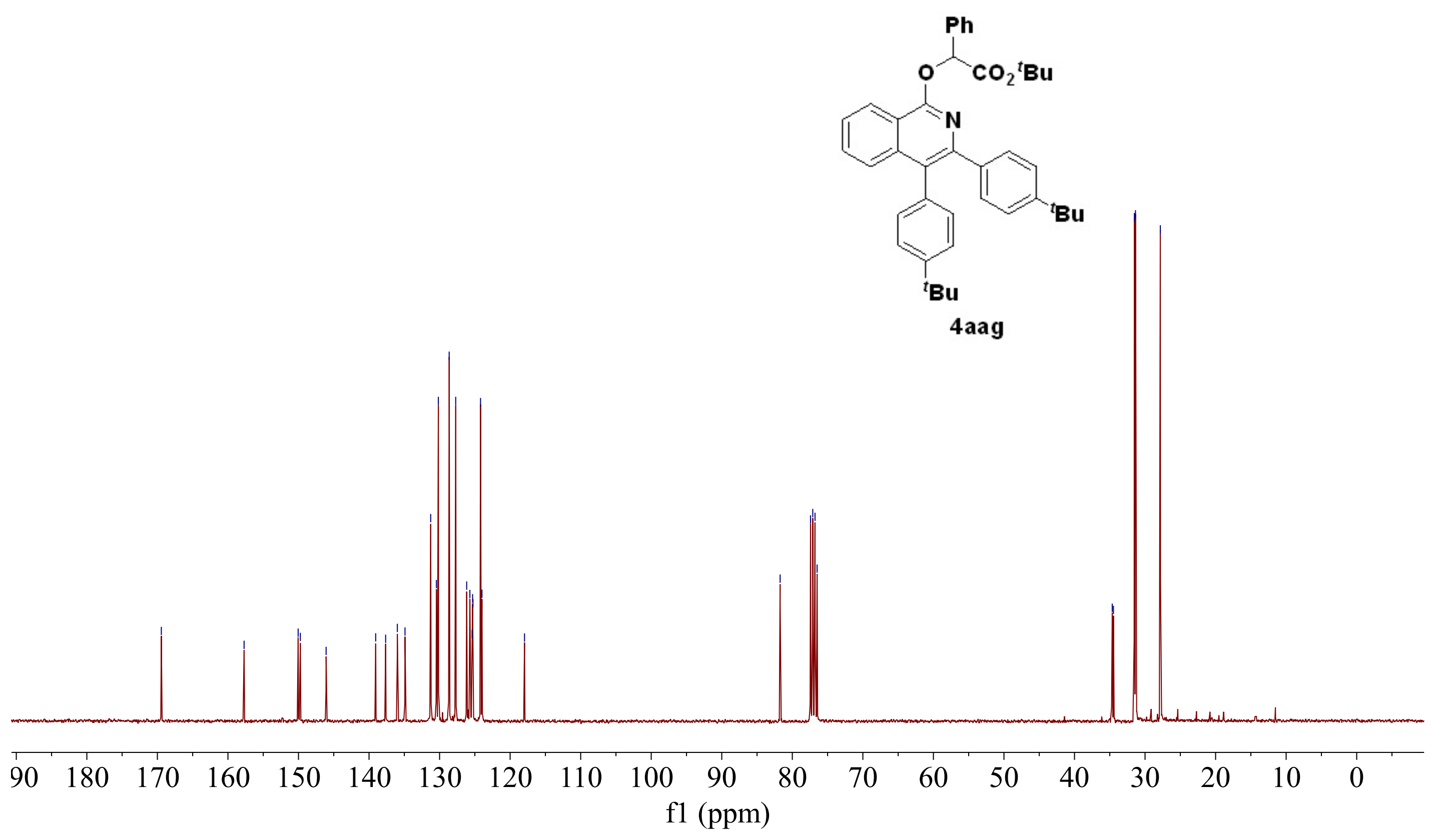




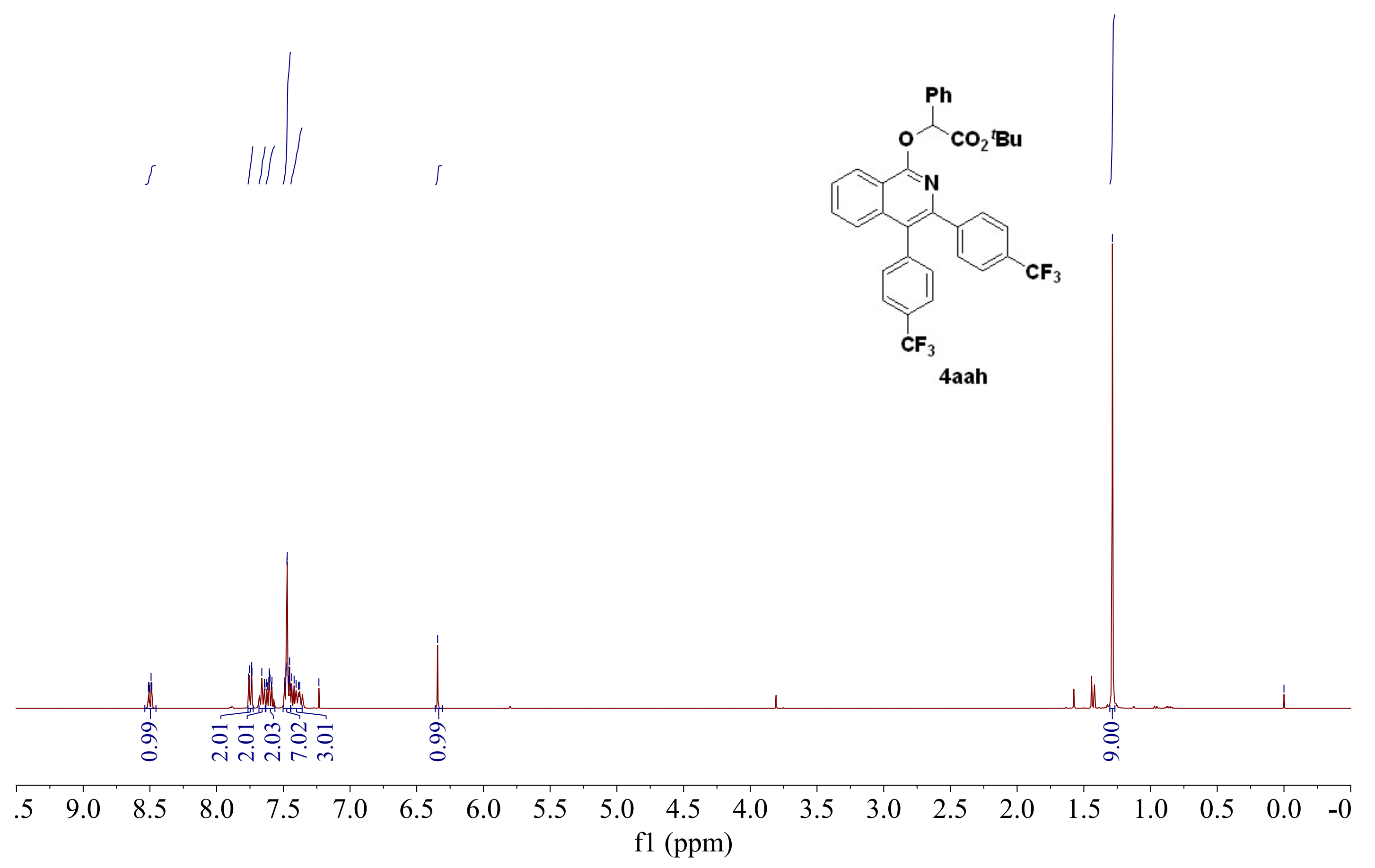




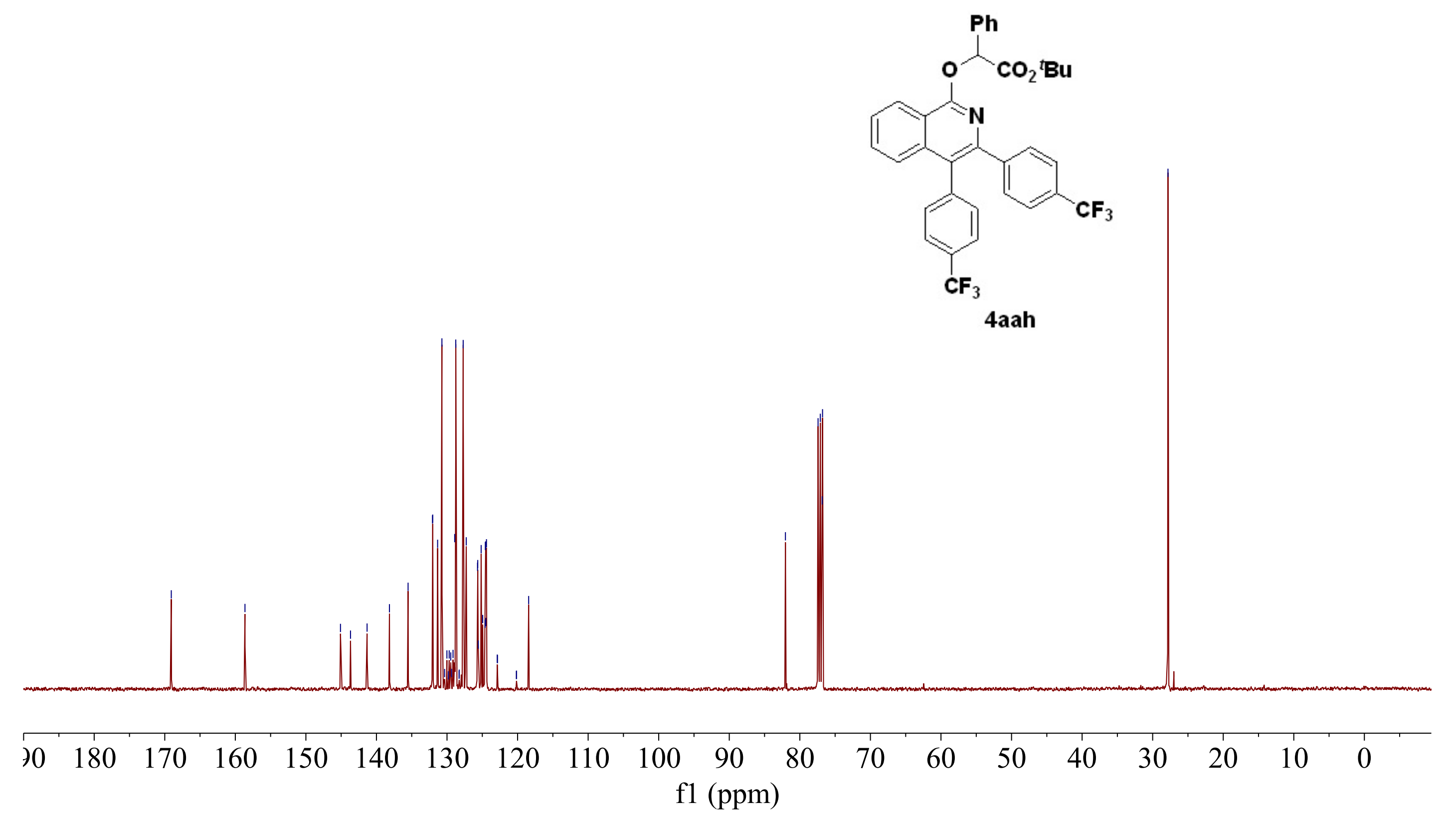




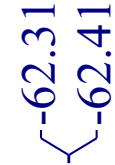

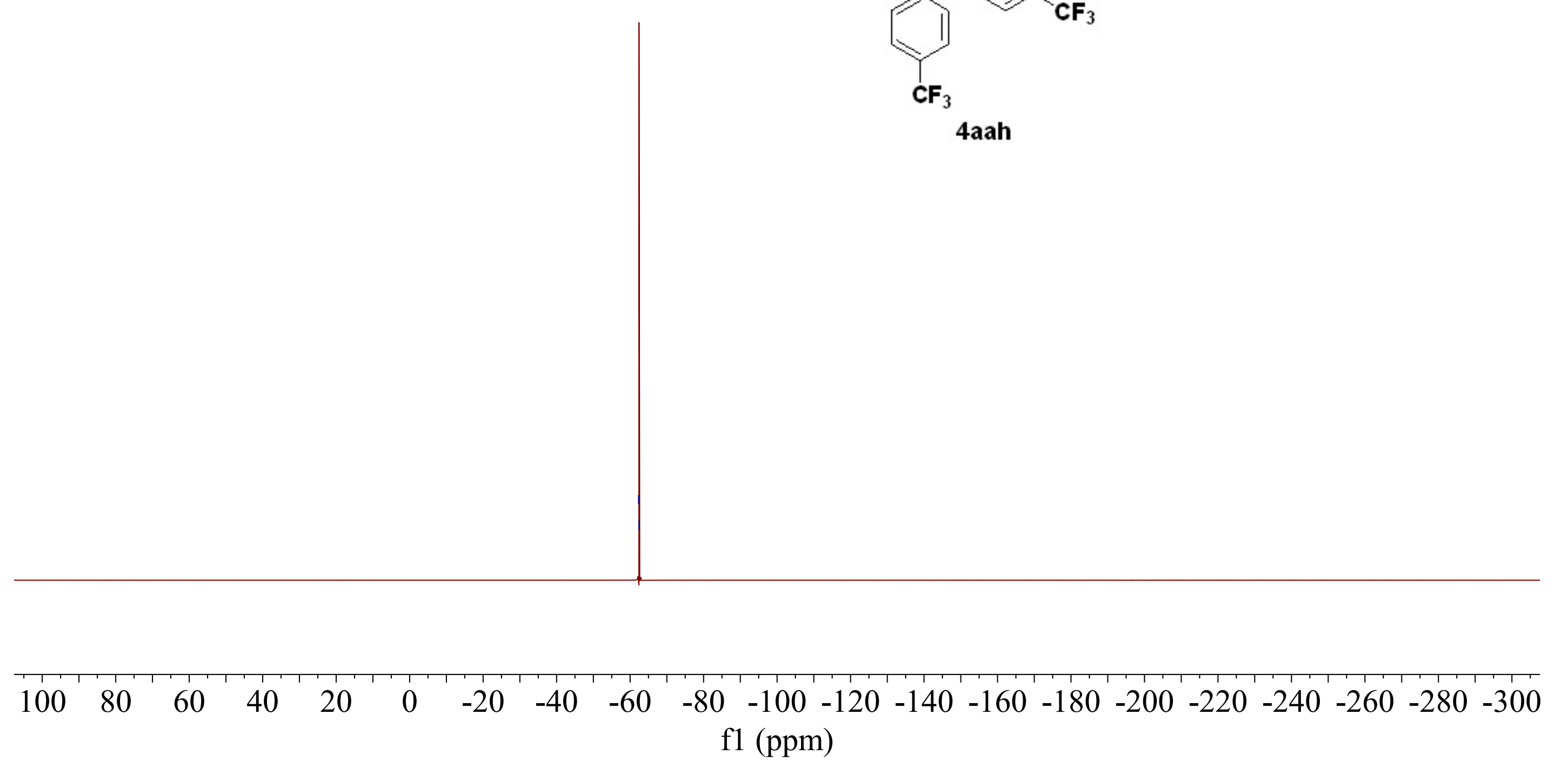



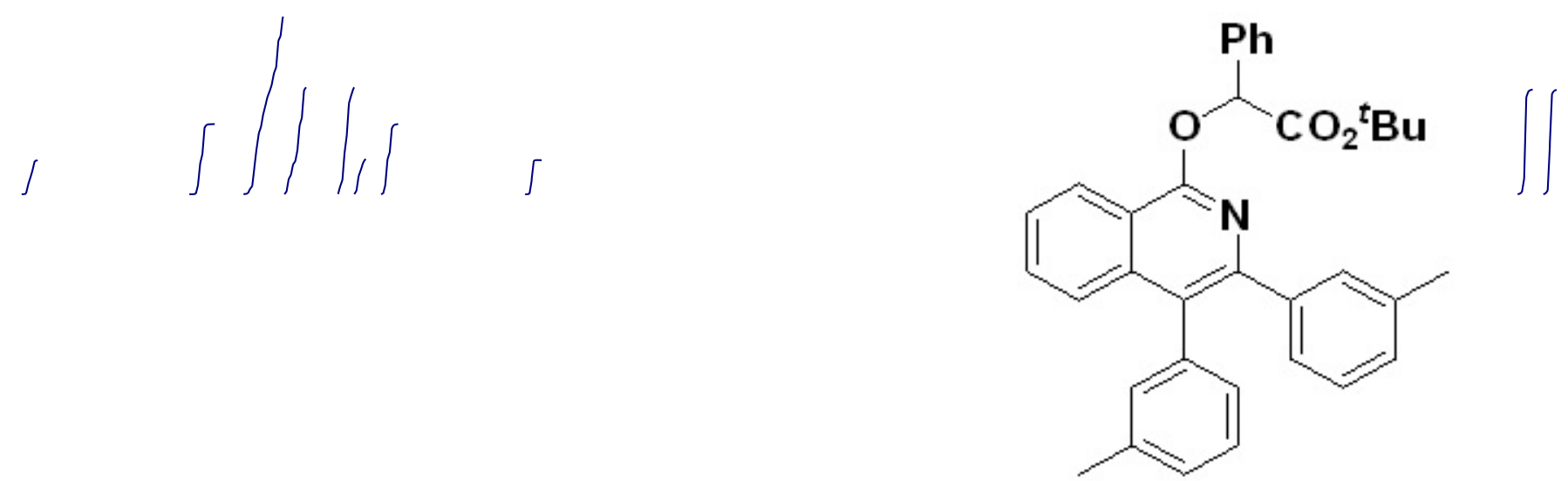

4aai

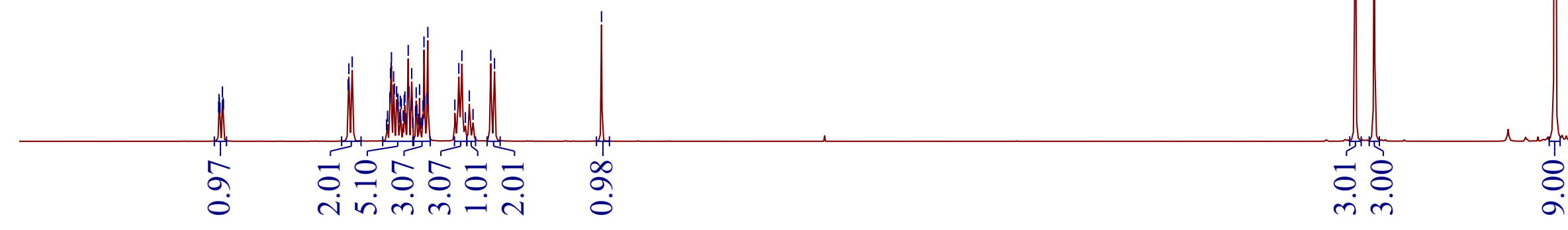

\begin{tabular}{lllllllllllllllllll}
\hline .5 & 9.0 & 8.5 & 8.0 & 7.5 & 7.0 & 6.5 & 6.0 & 5.5 & $\begin{array}{c}5.0 \\
\text { fl }(\mathrm{ppm})\end{array}$ & $\begin{array}{c}4.5 \\
4.0\end{array}$ & 3.5 & 3.0 & 2.5 & 2.0 & 1.5 & 1.0 & 0.5 & 0.0
\end{tabular}



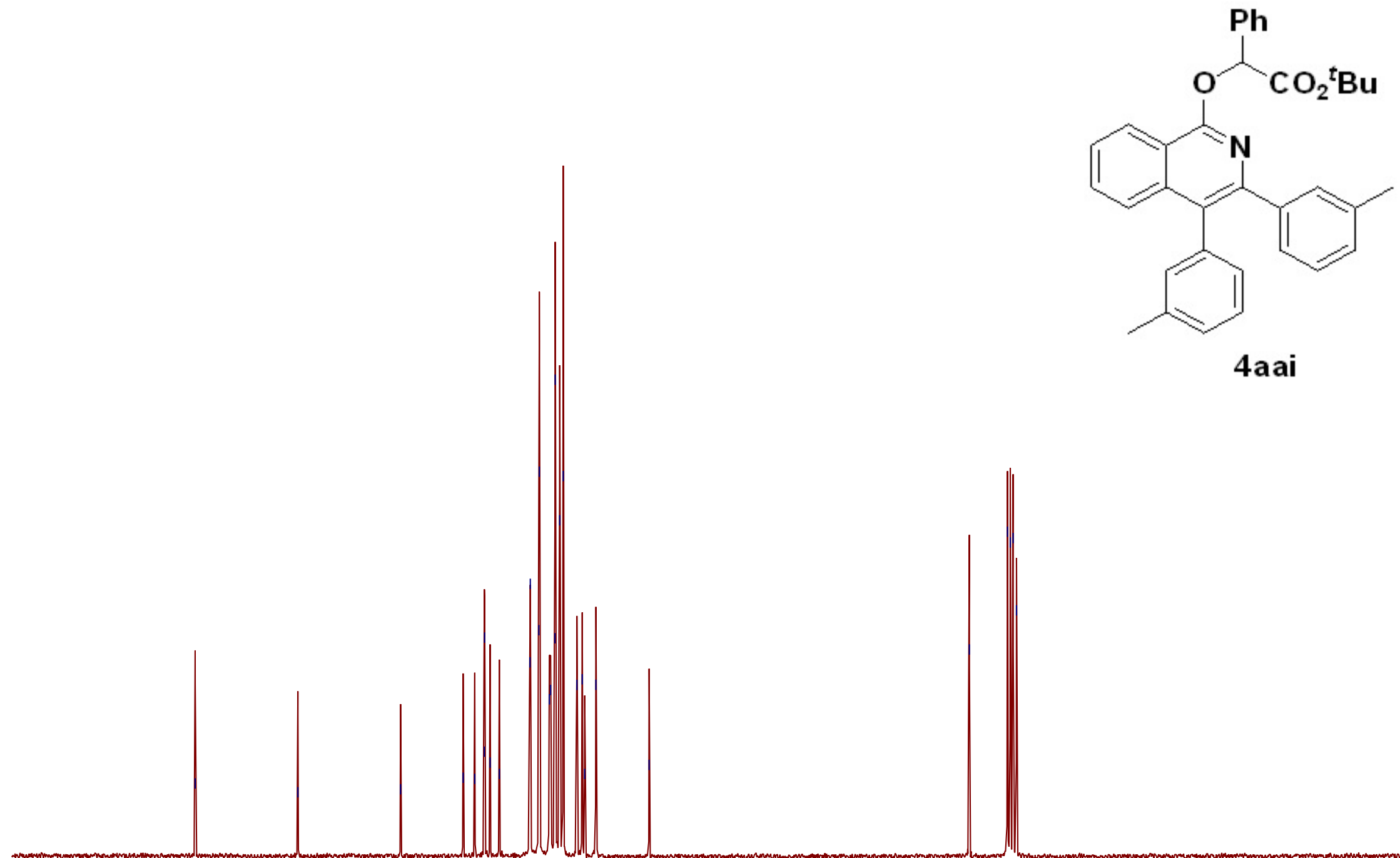

4aai 

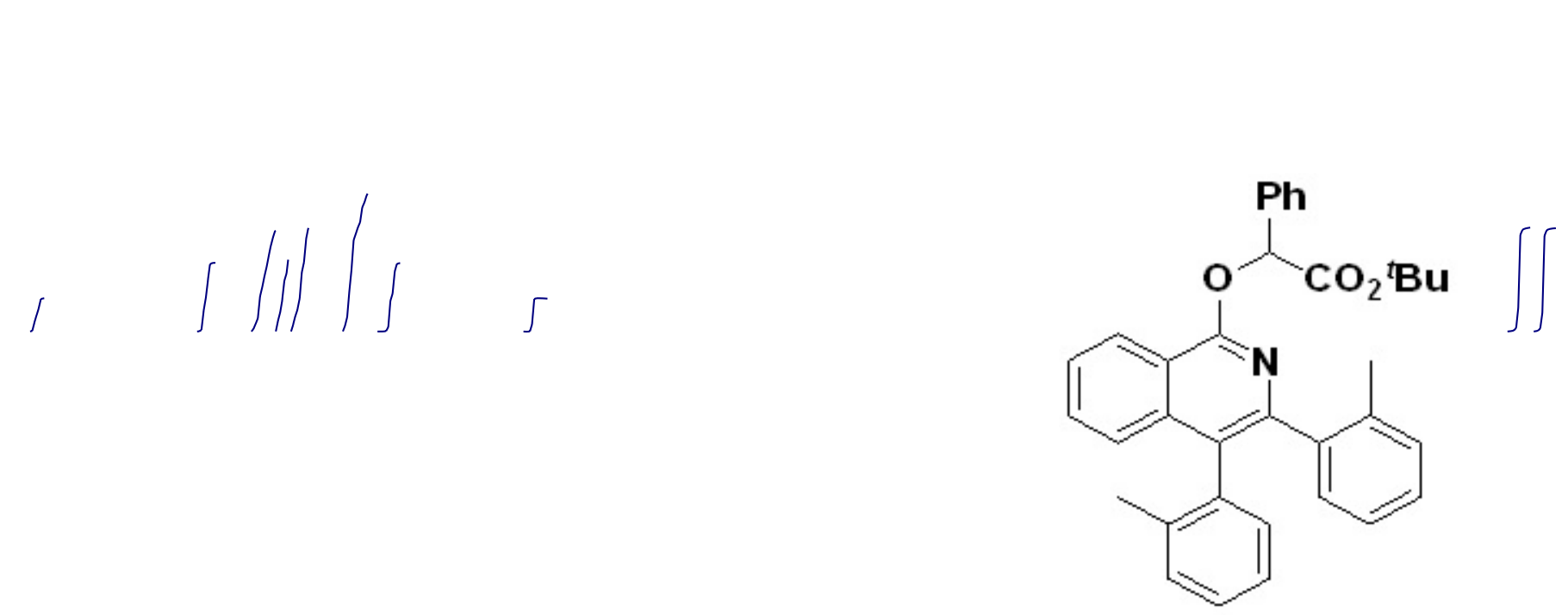

4aaj

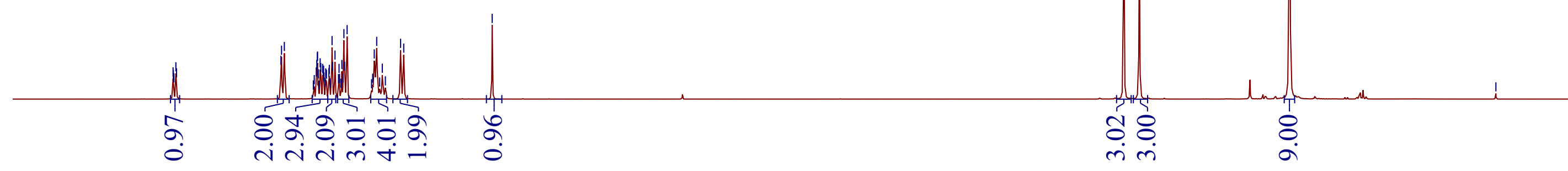

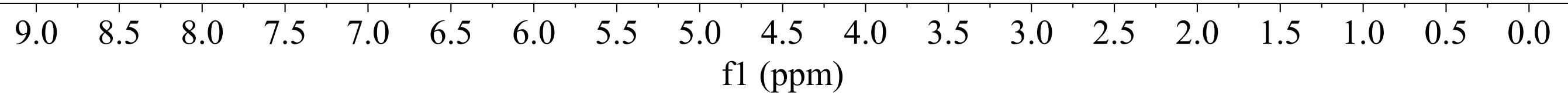




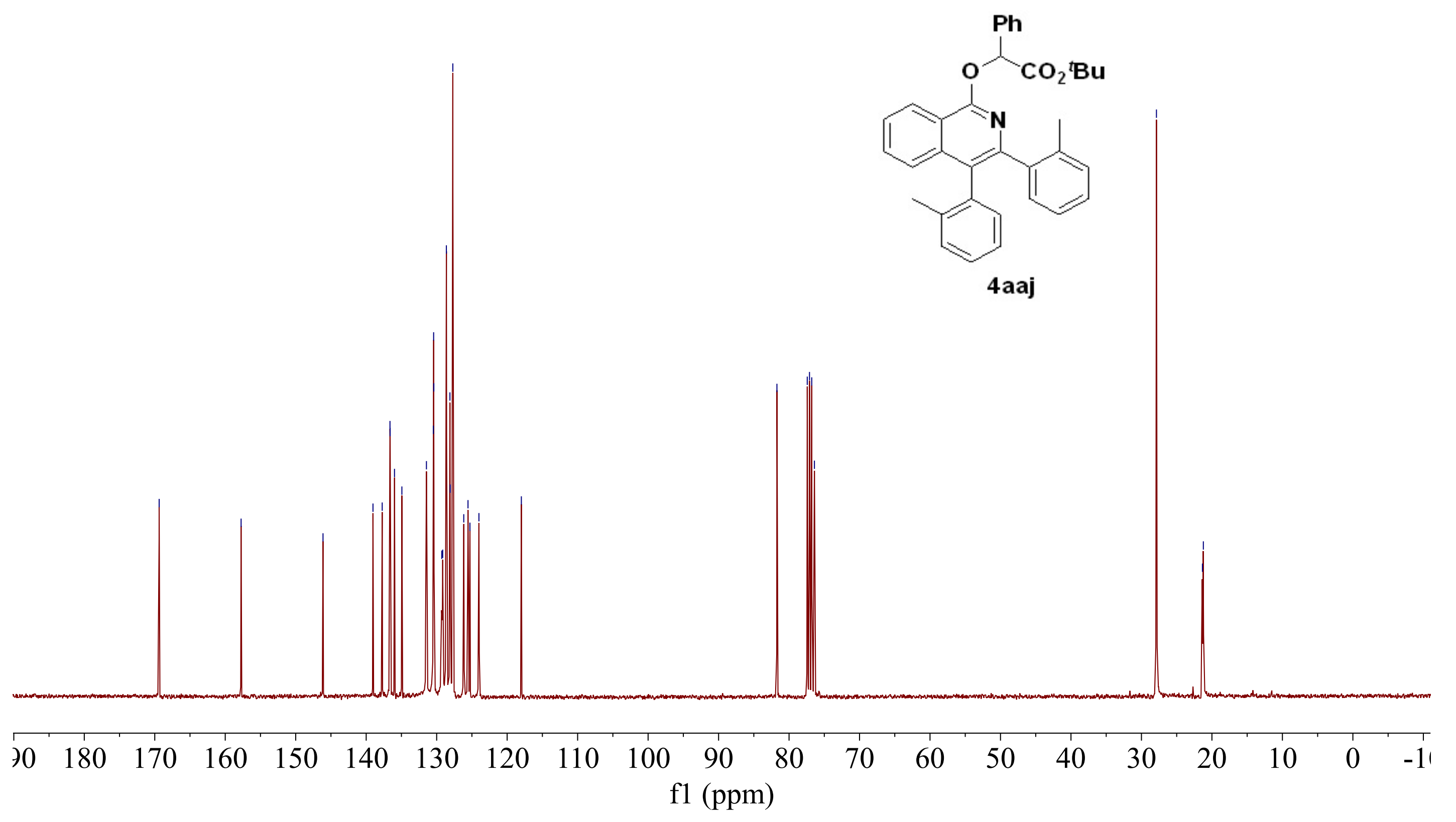




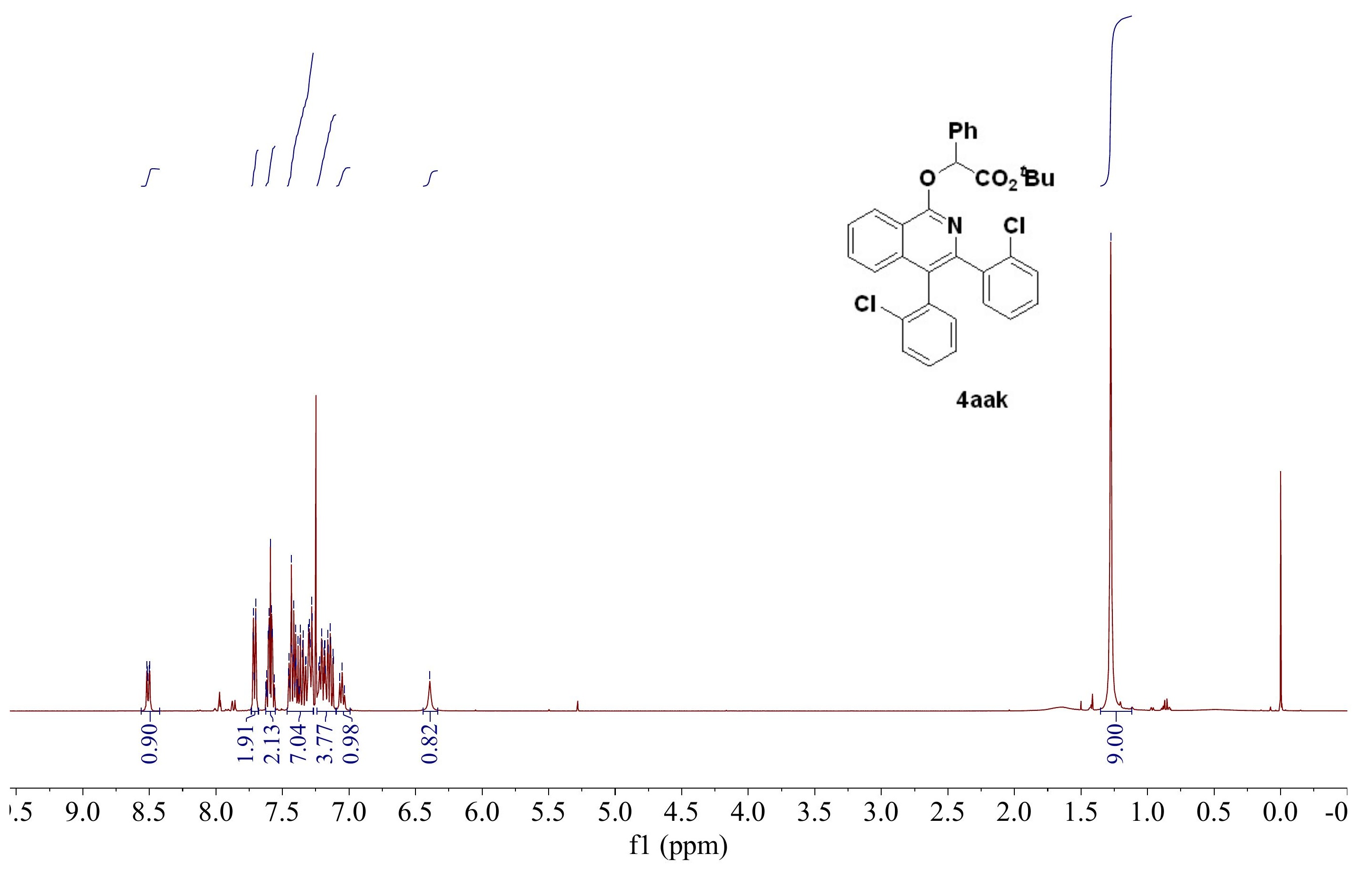




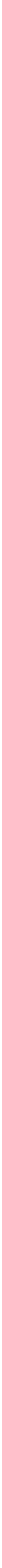



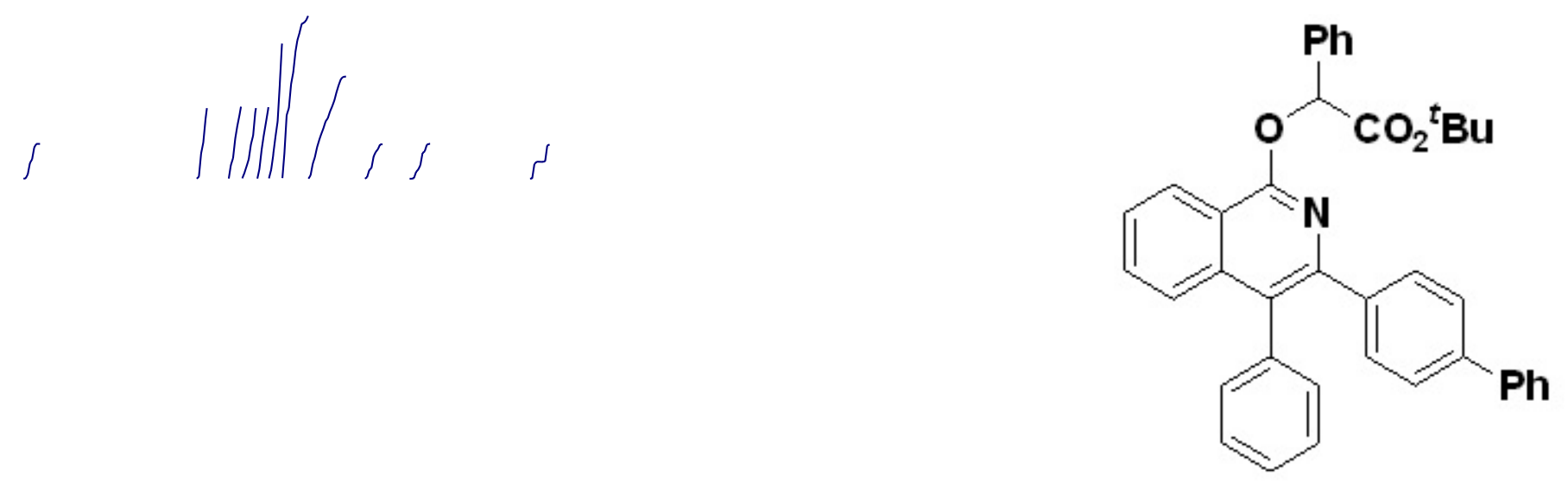

4aal + 4aal': regiomers ratio (1:1)

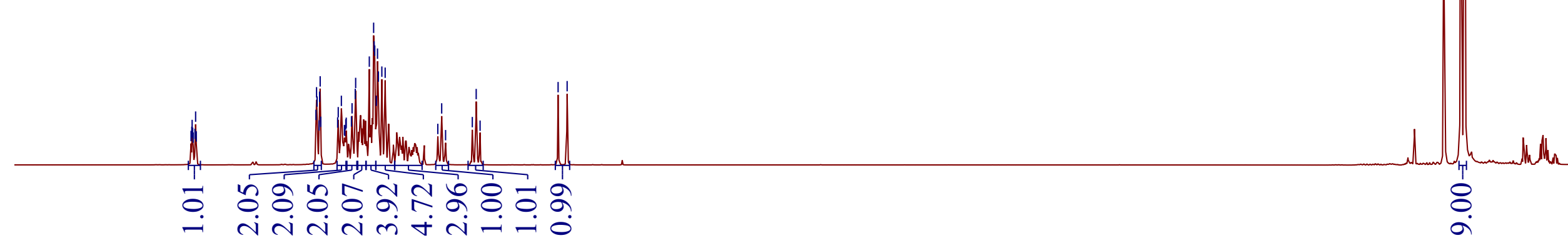

$\begin{array}{cccccccccccccccccc}9.0 & 8.5 & 8.0 & 7.5 & 7.0 & 6.5 & 6.0 & 5.5 & \begin{array}{c}5.0 \\ \mathrm{fl}(\mathrm{ppm})\end{array} & \begin{array}{c}4.5 \\ 4.0\end{array} & 3.5 & 3.0 & 2.5 & 2.0 & 1.5 & 1.0 & 0.5 & 0.0\end{array}$




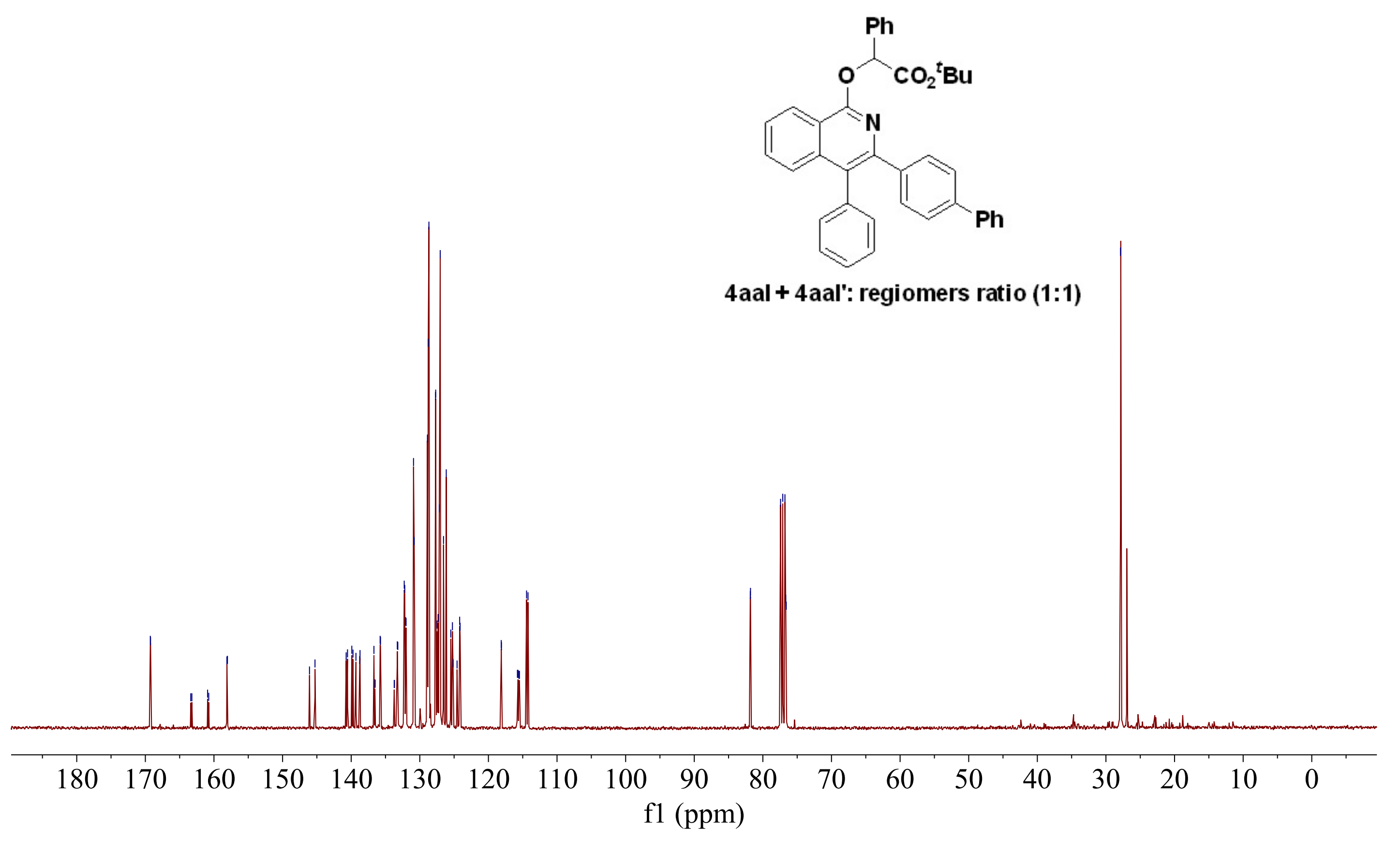




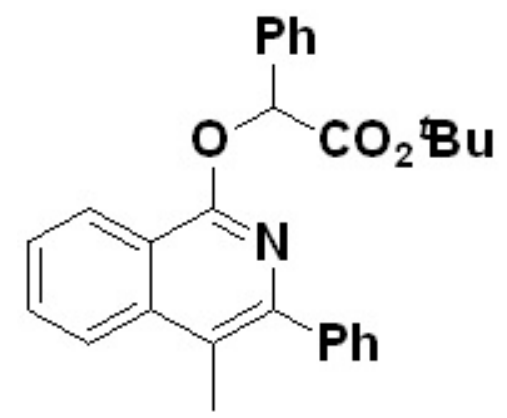

4aam

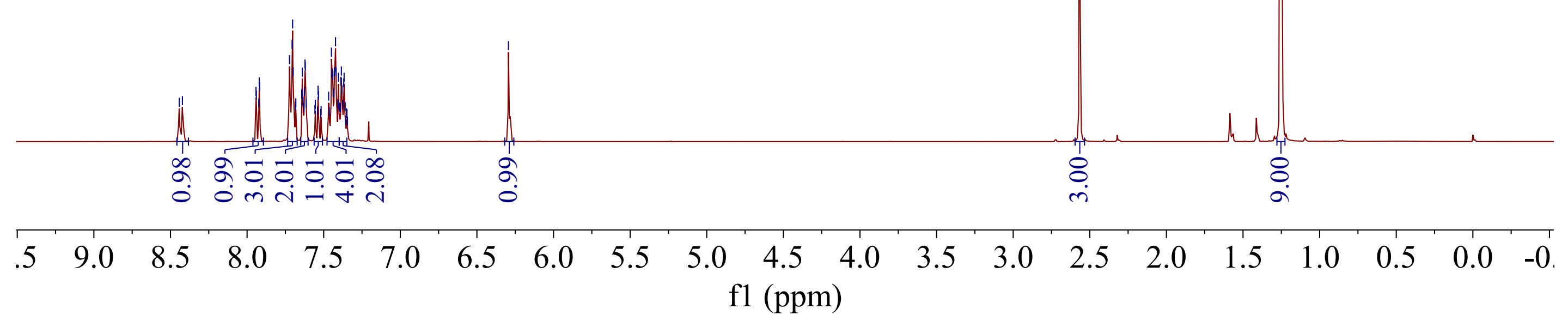




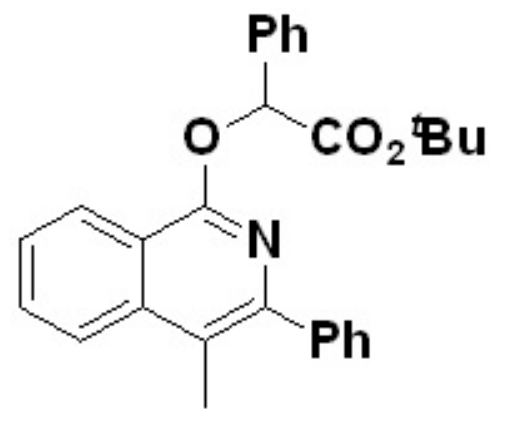

\section{4aam}

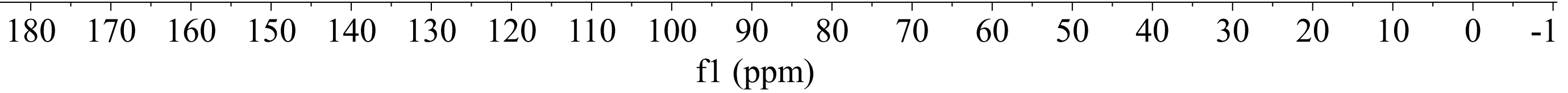




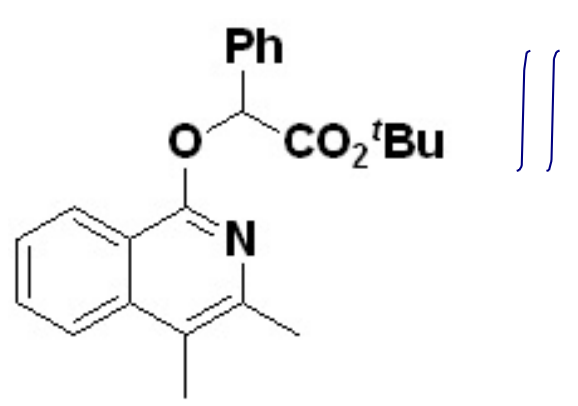

4aan

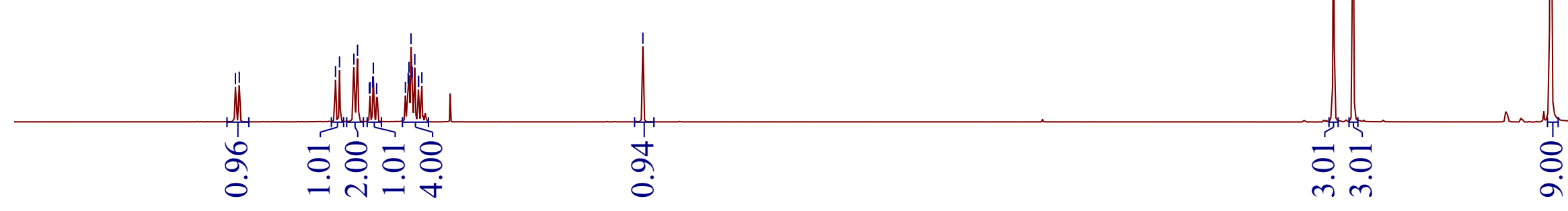

$\begin{array}{llllll}8.5 & 8.0 & 7.5 & 7.0 & 6.5 & 6.0\end{array}$

$6.0 \quad 5.5$




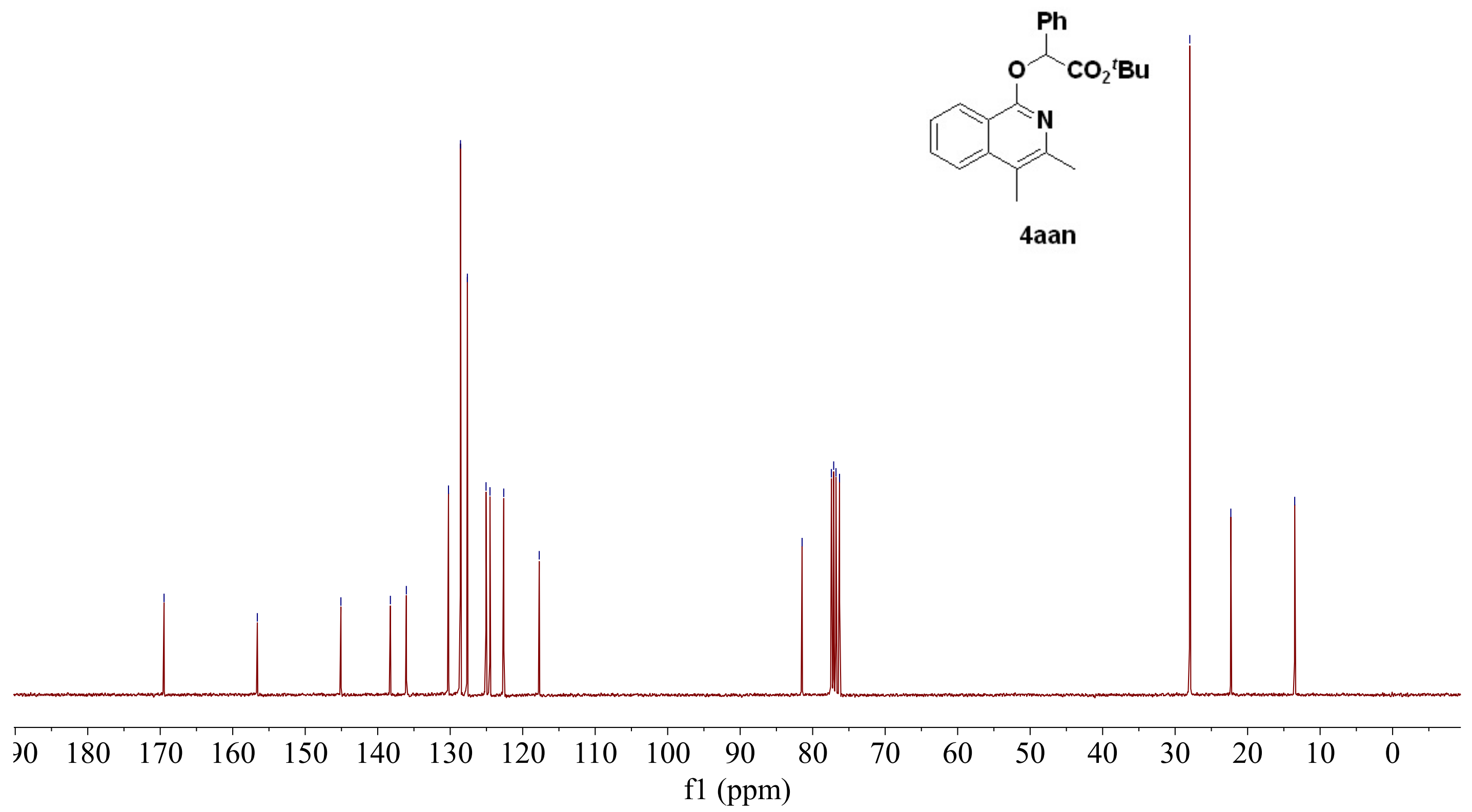



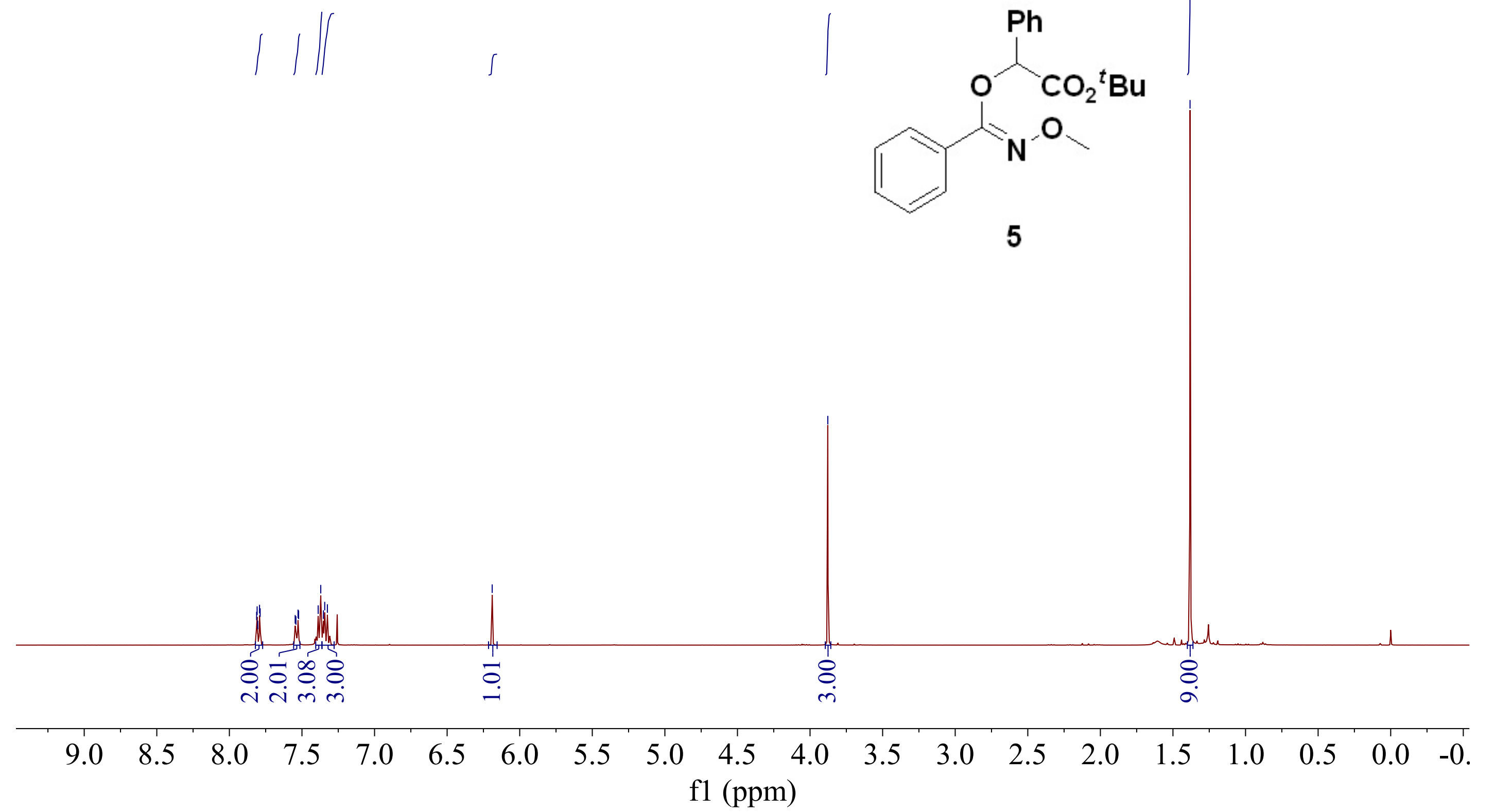


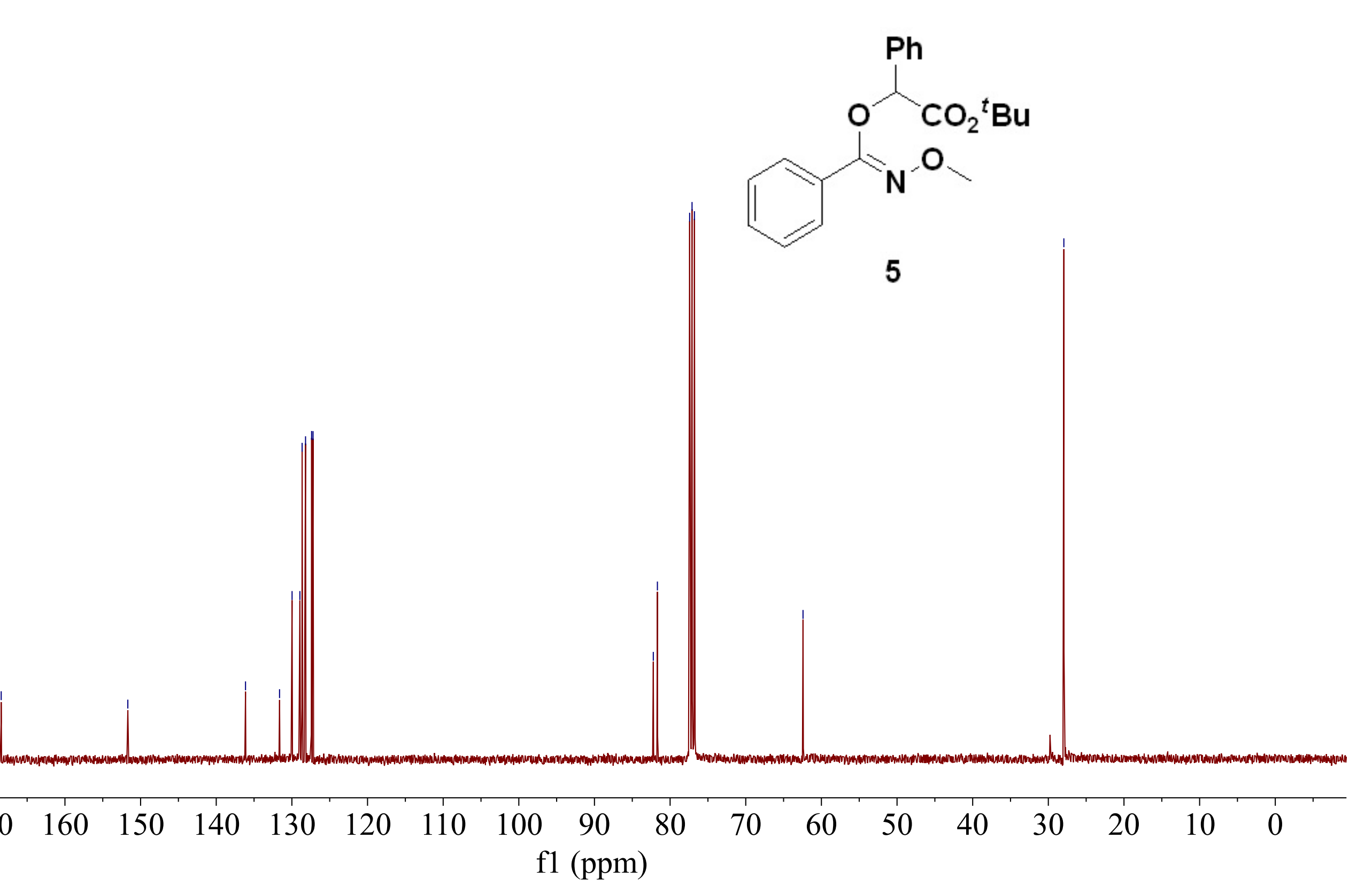



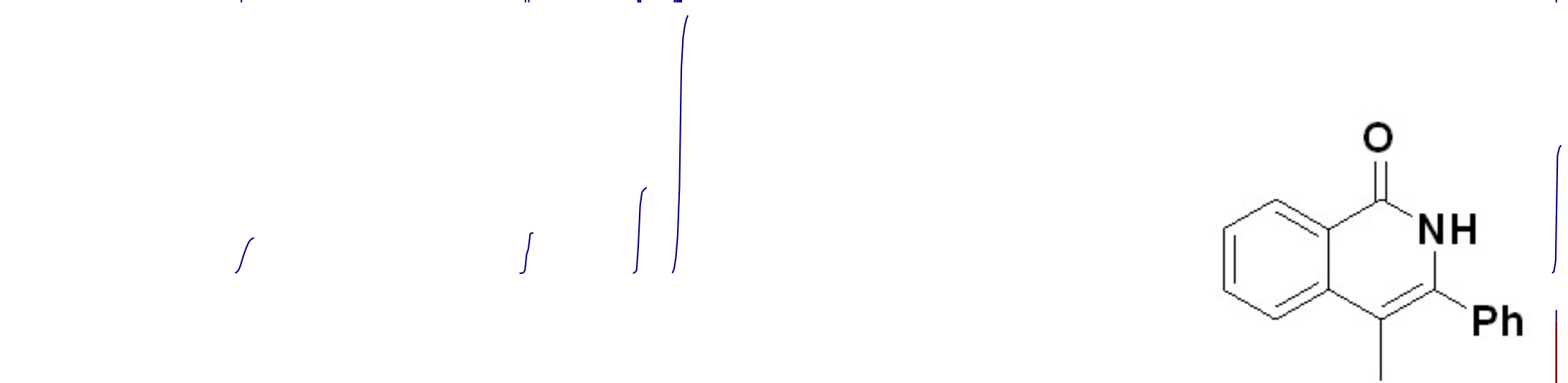

8

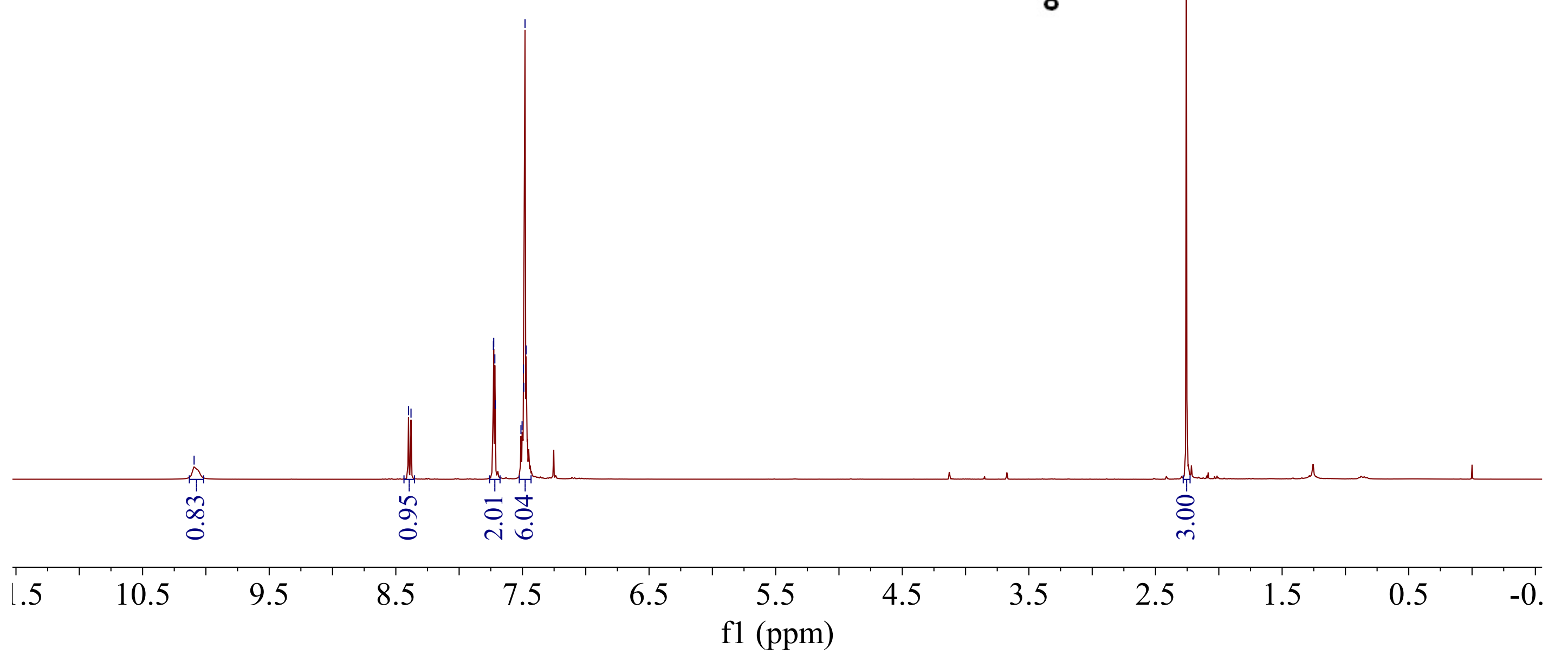




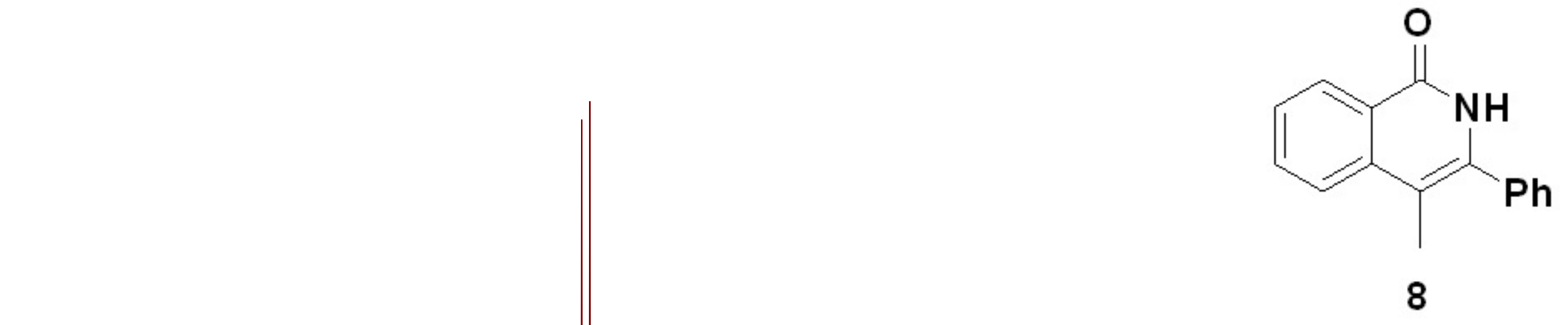

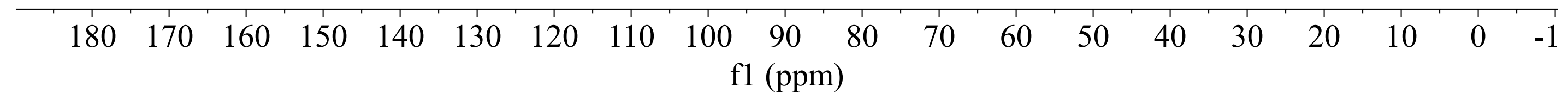



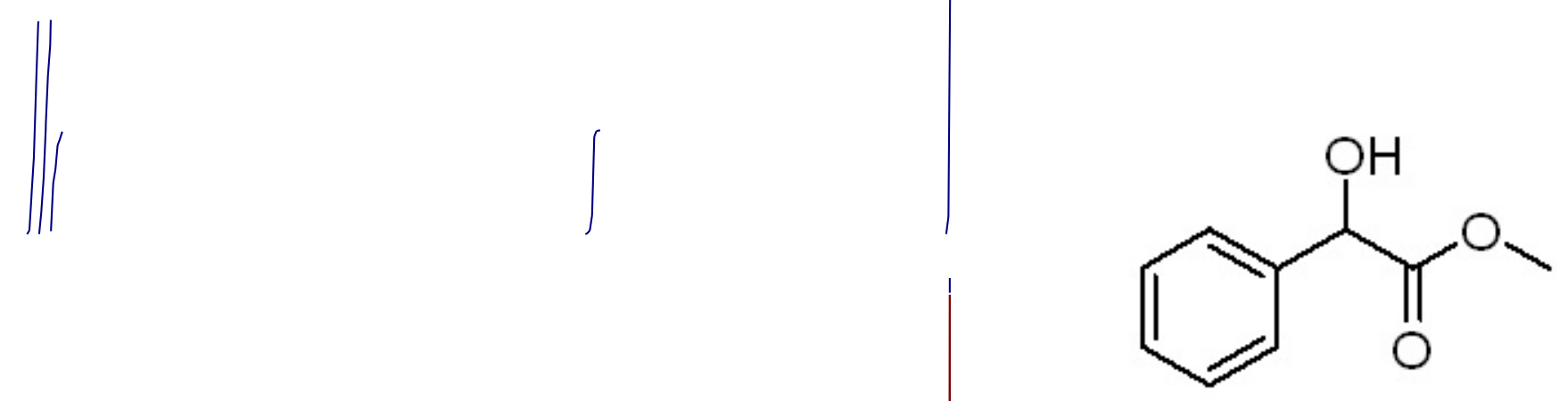

10

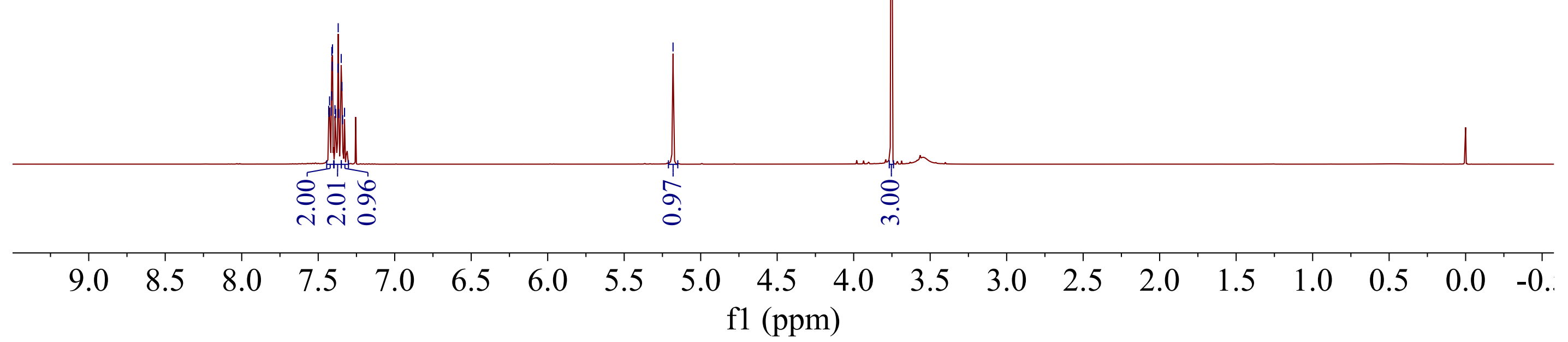




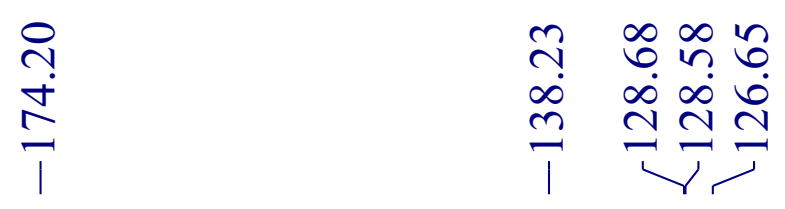

ㄱำ

NN N

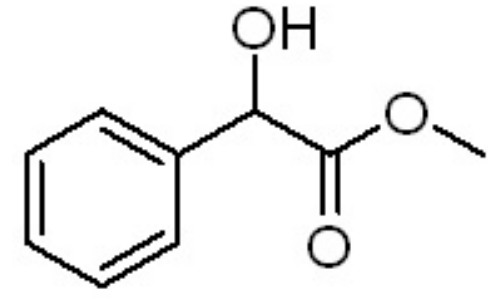

10 


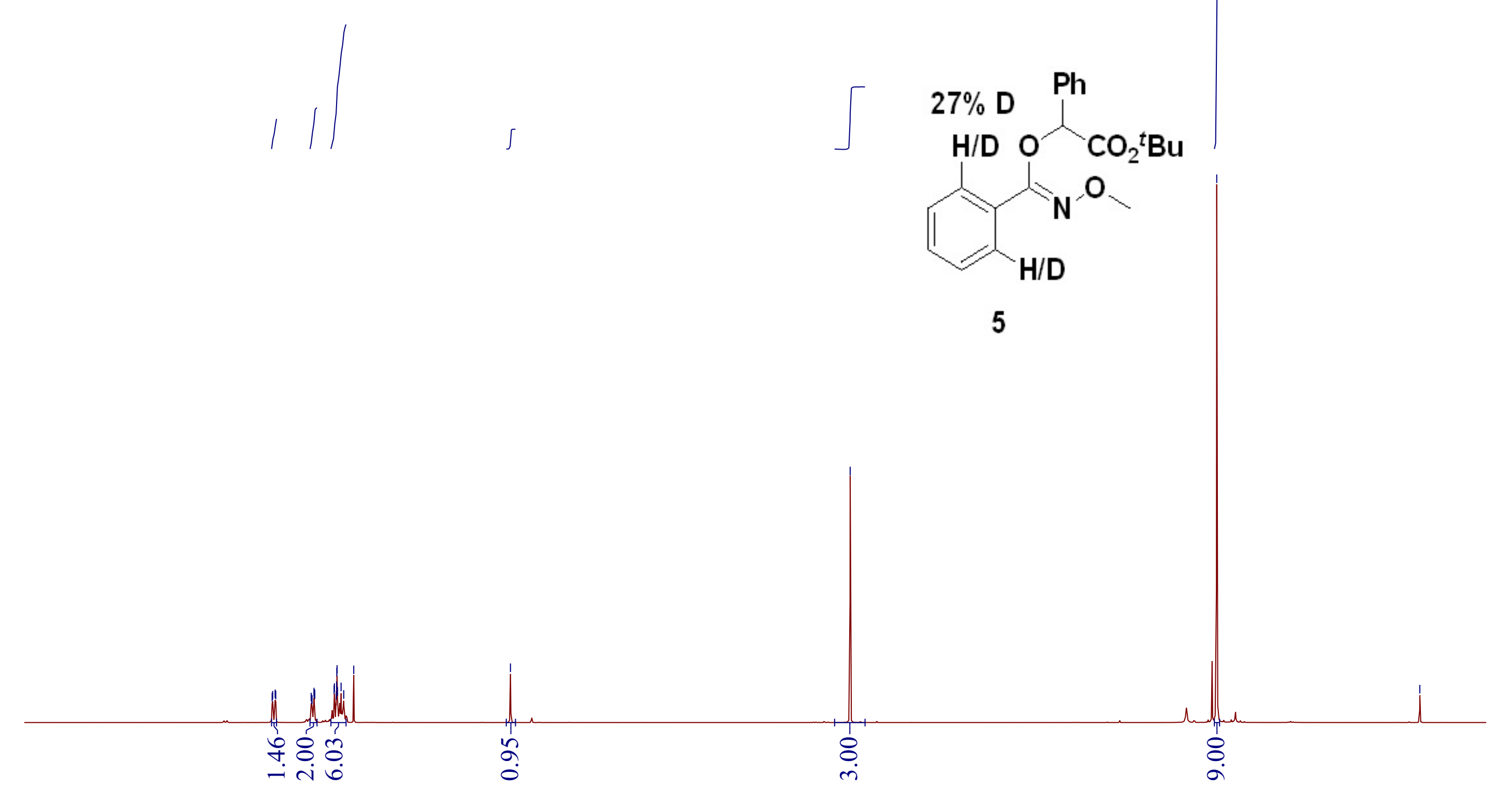

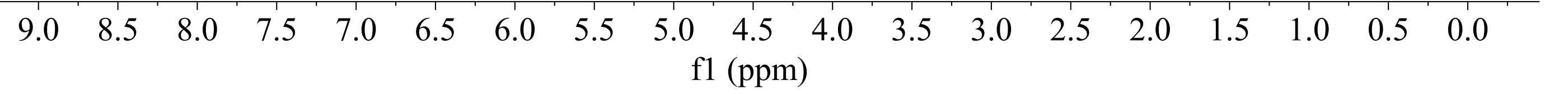



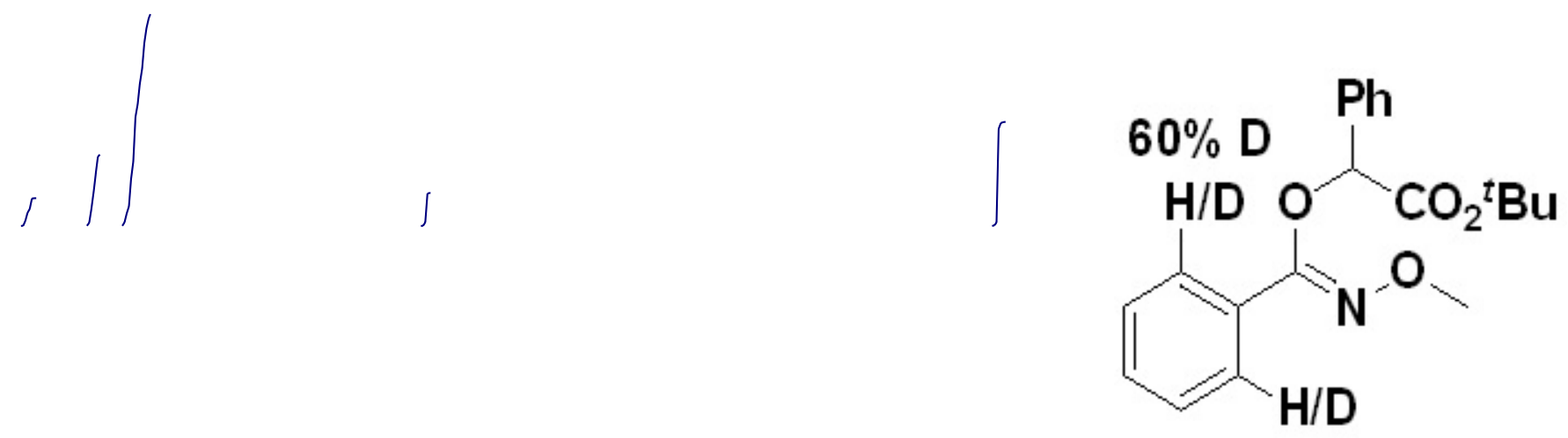

5

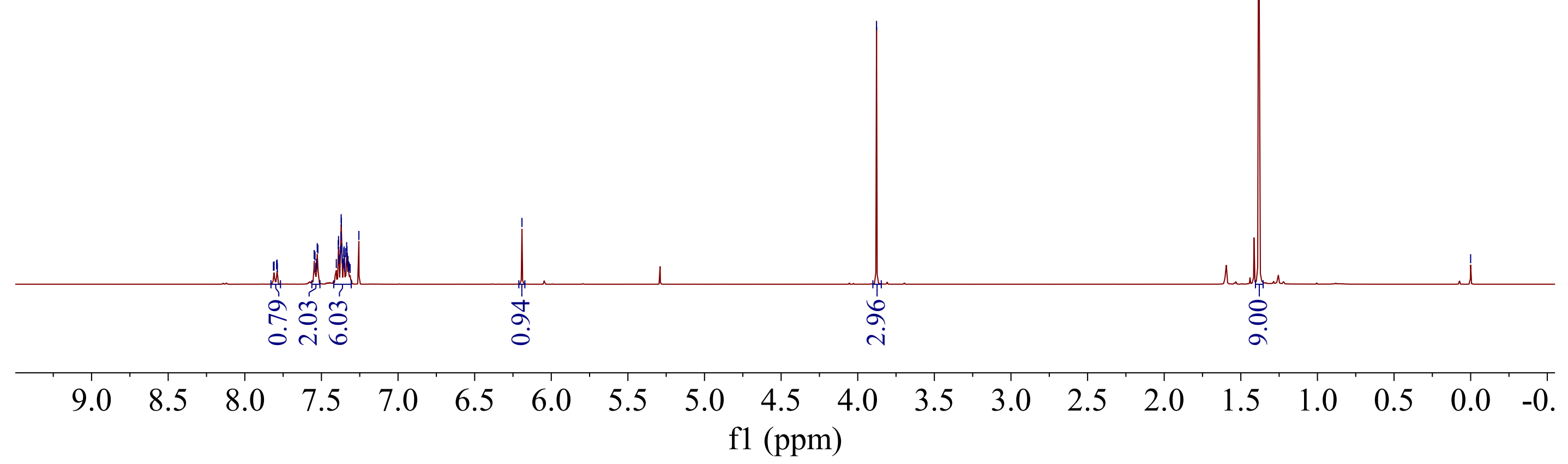




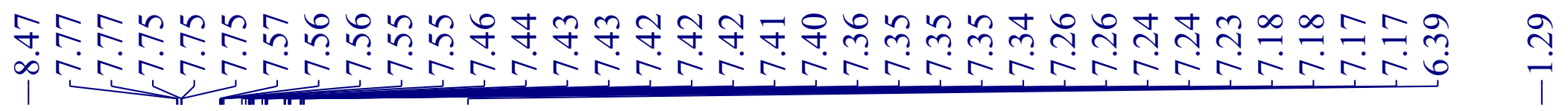
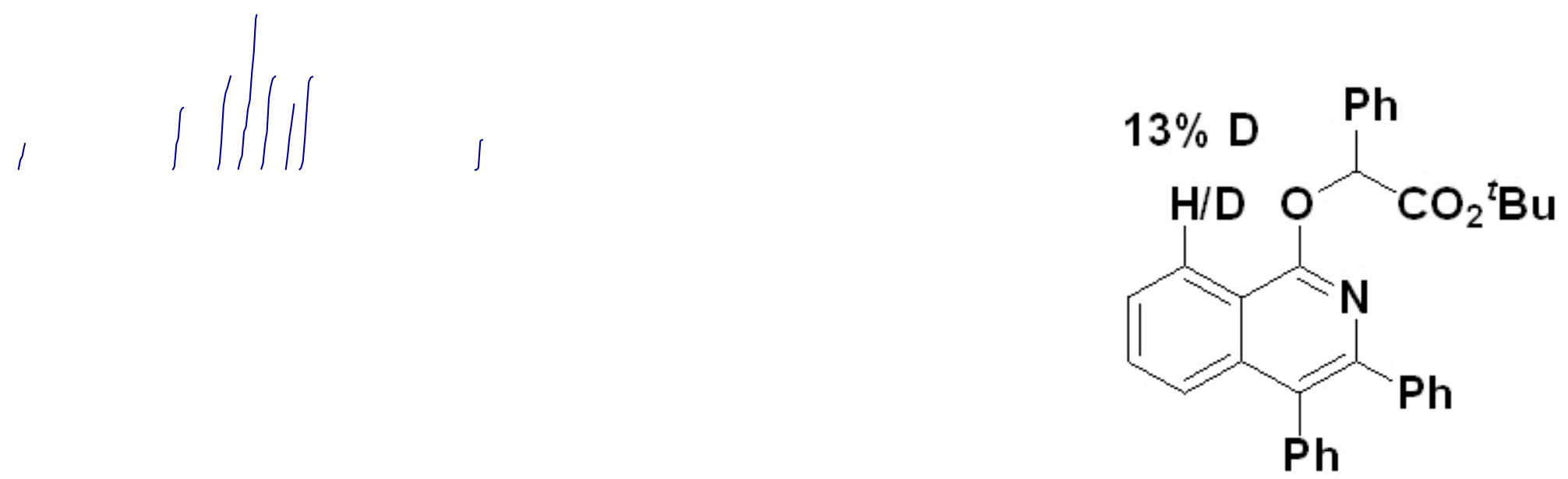

4aaa

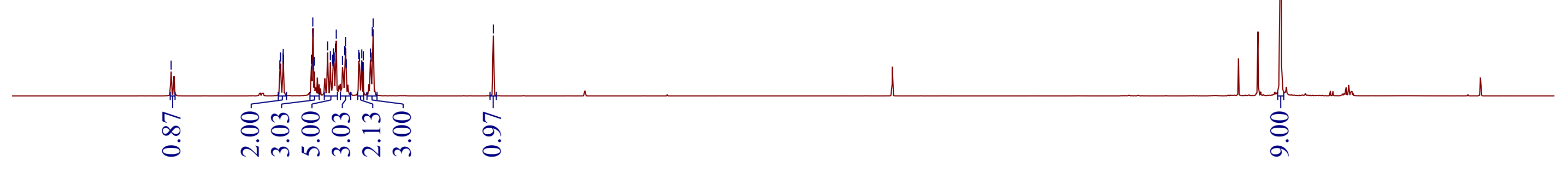

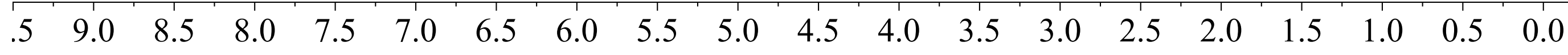
f1 (ppm) 


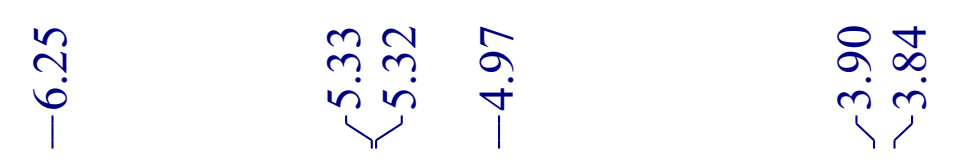

Reaction time: $0.05 \mathrm{~h}$ (3.0 min)
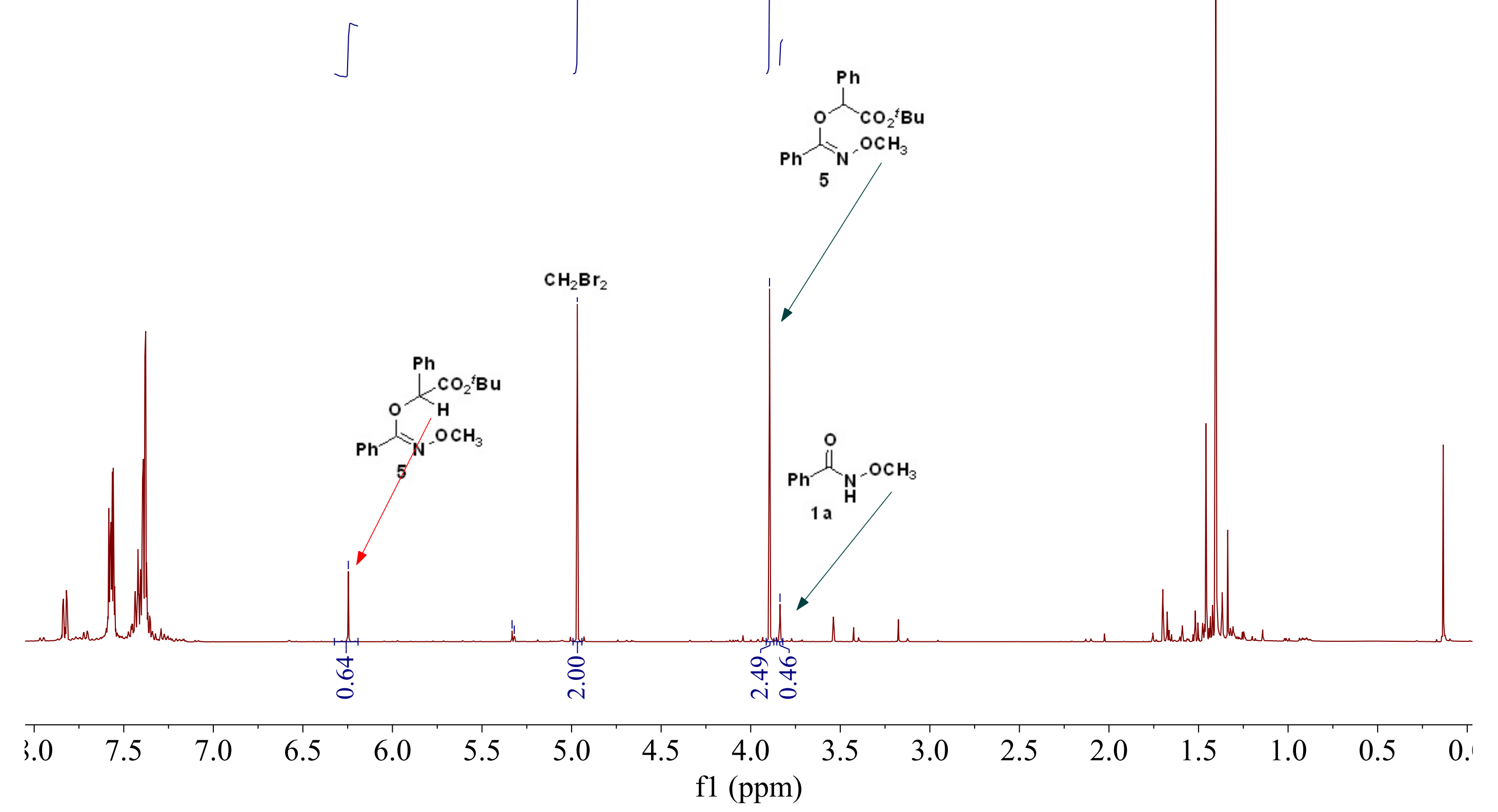


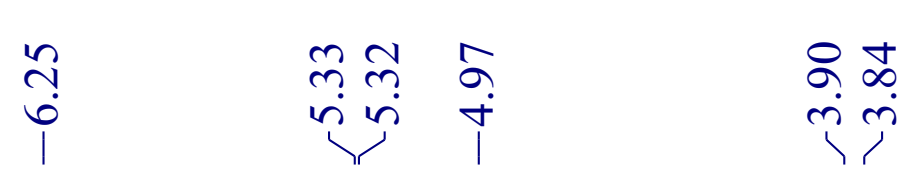

Reaction time: $0.17 \mathrm{~h}$ (10.0 min)

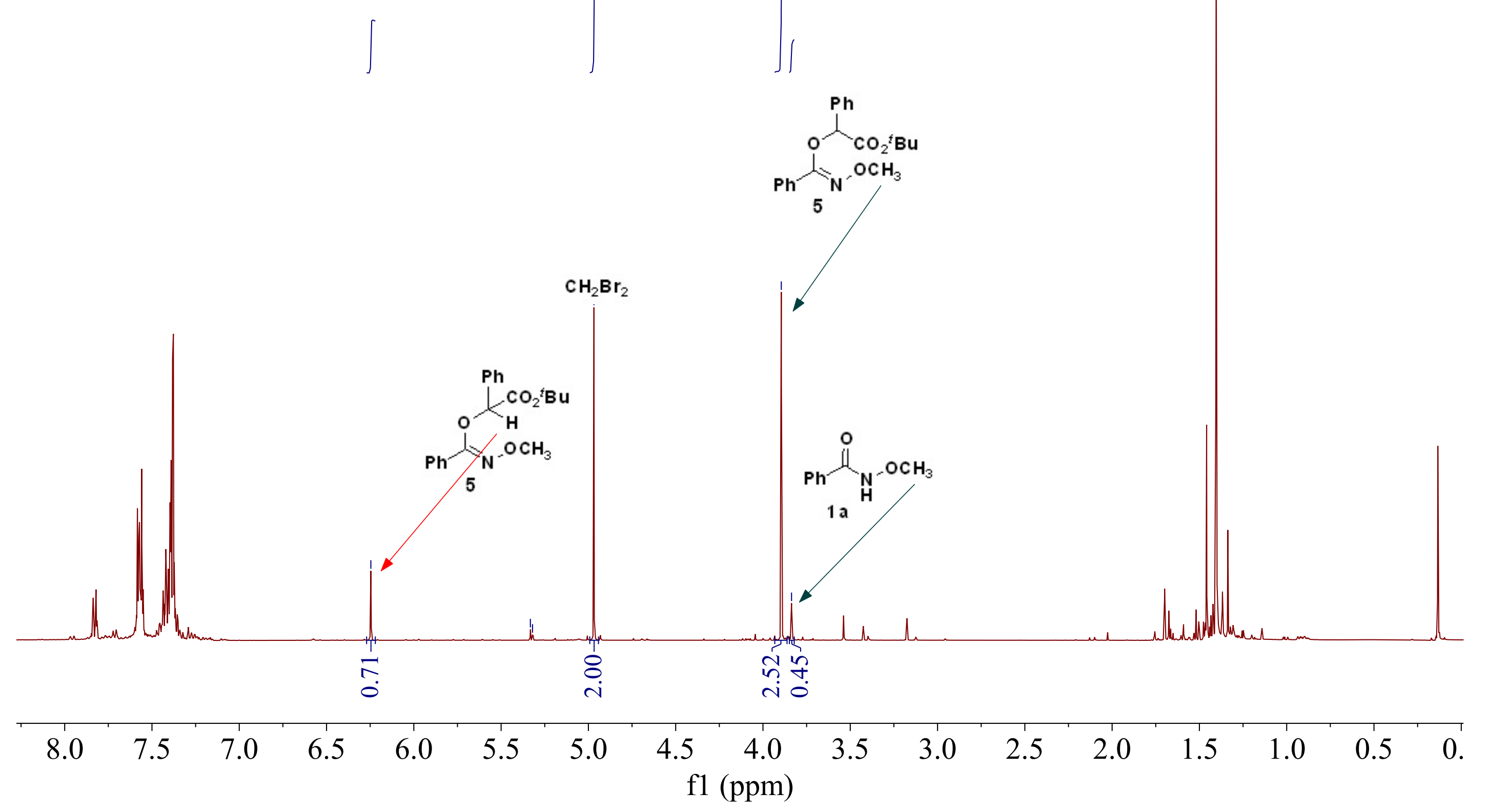




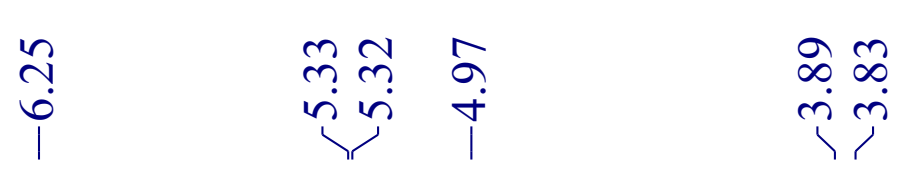

Reaction time: $0.33 \mathrm{~h}$ (20.0 min)

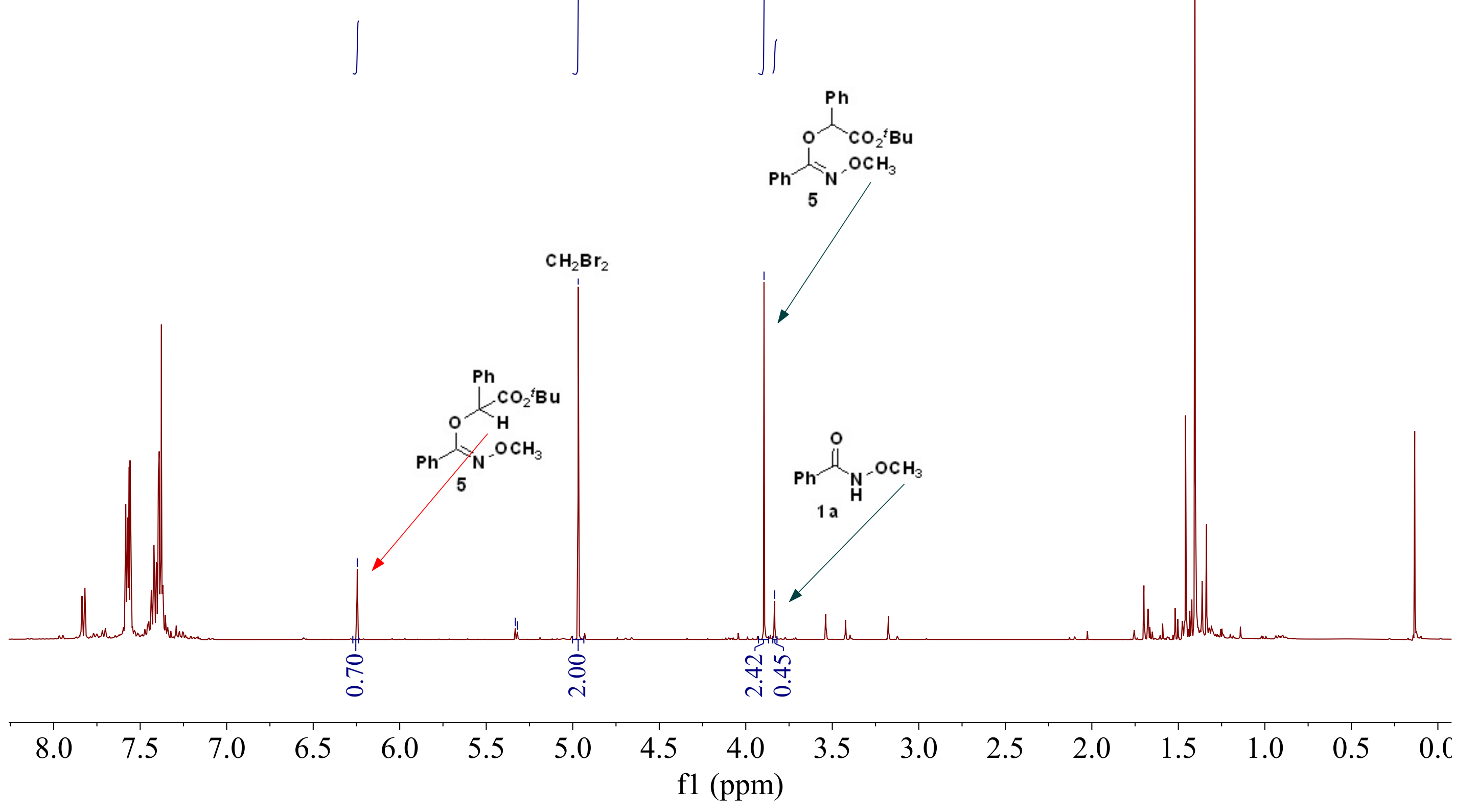




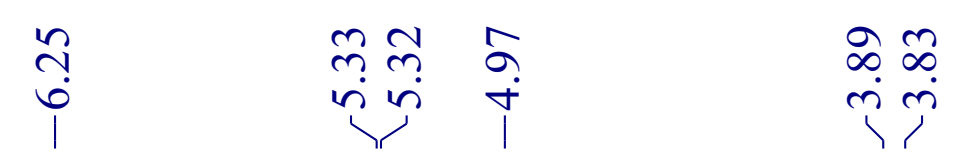

Reaction time: $0.5 \mathrm{~h}$ (30.0 min)

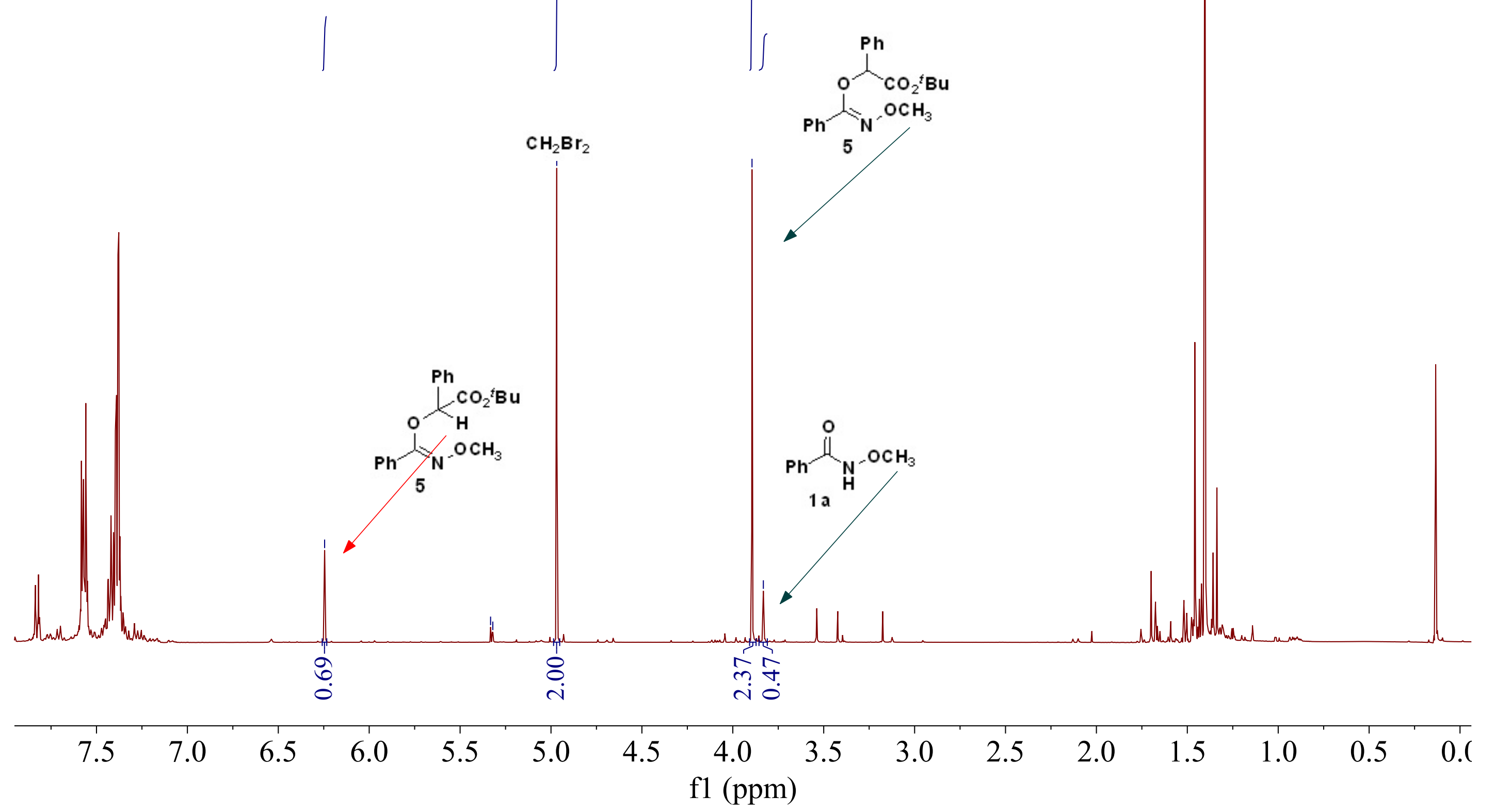




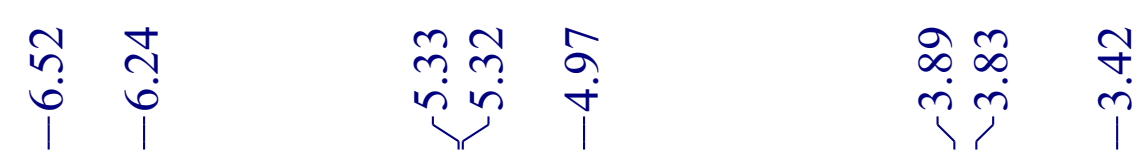

Reaction time: $0.67 \mathrm{~h}$ (40.0 min)

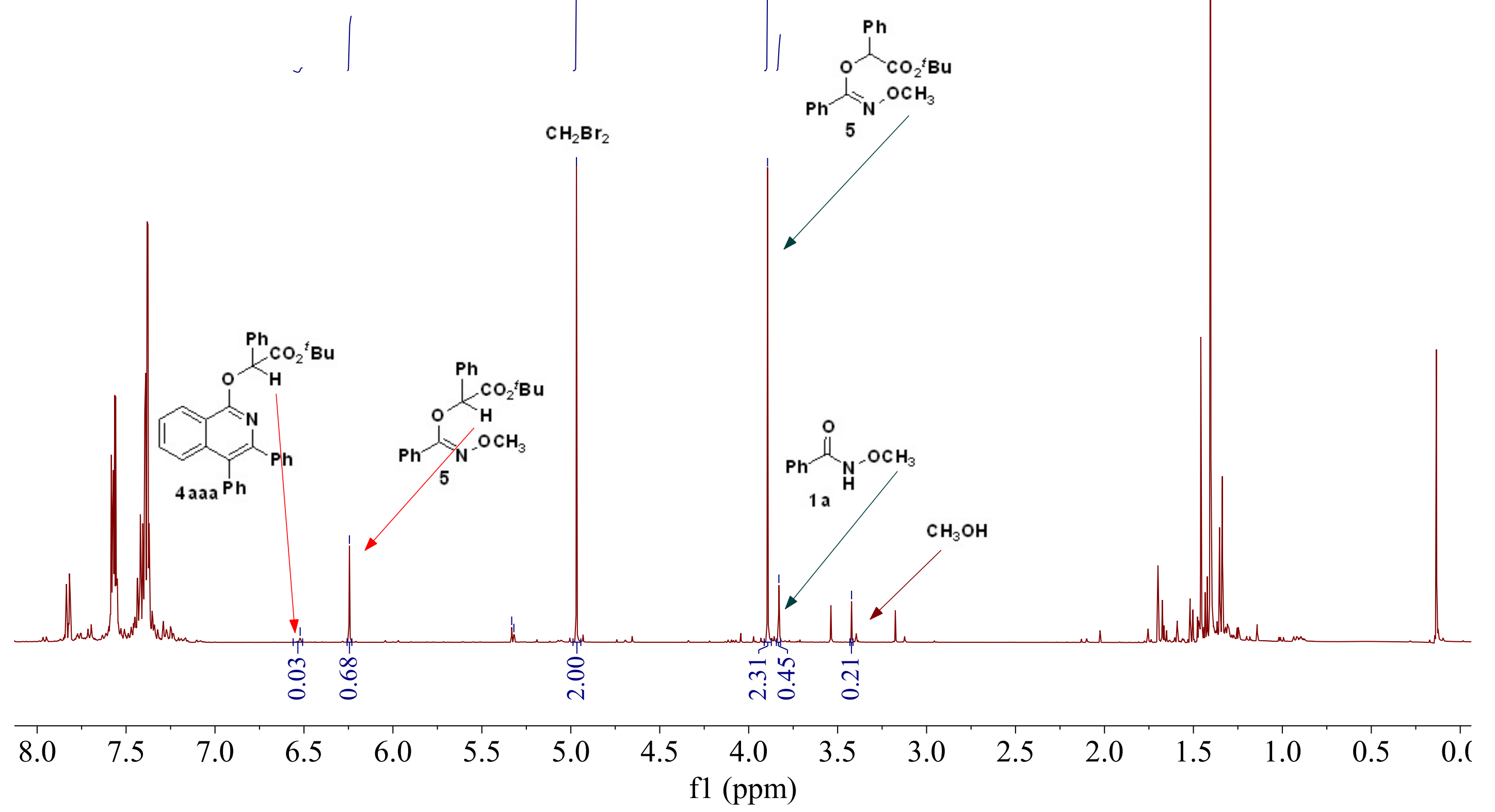




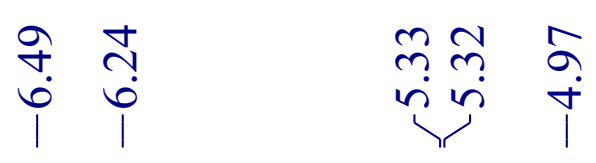

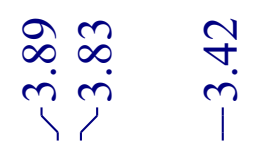

Reaction time: $1.0 \mathrm{~h}$ (60.0 min)

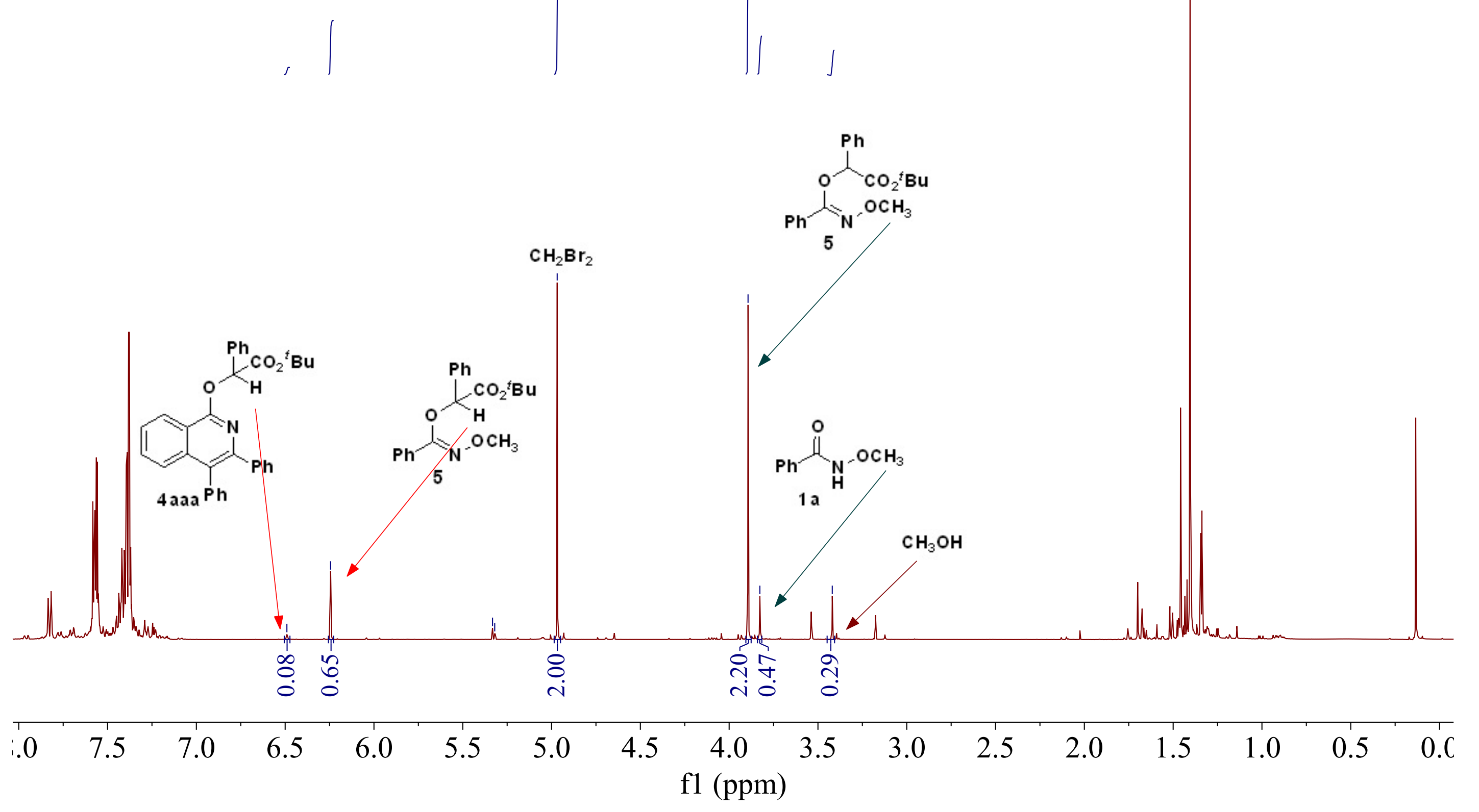




$$
\begin{array}{ll}
\nexists & \multirow{J}{*}{} \\
i & i
\end{array}
$$

Reaction time: $1.67 \mathrm{~h}$
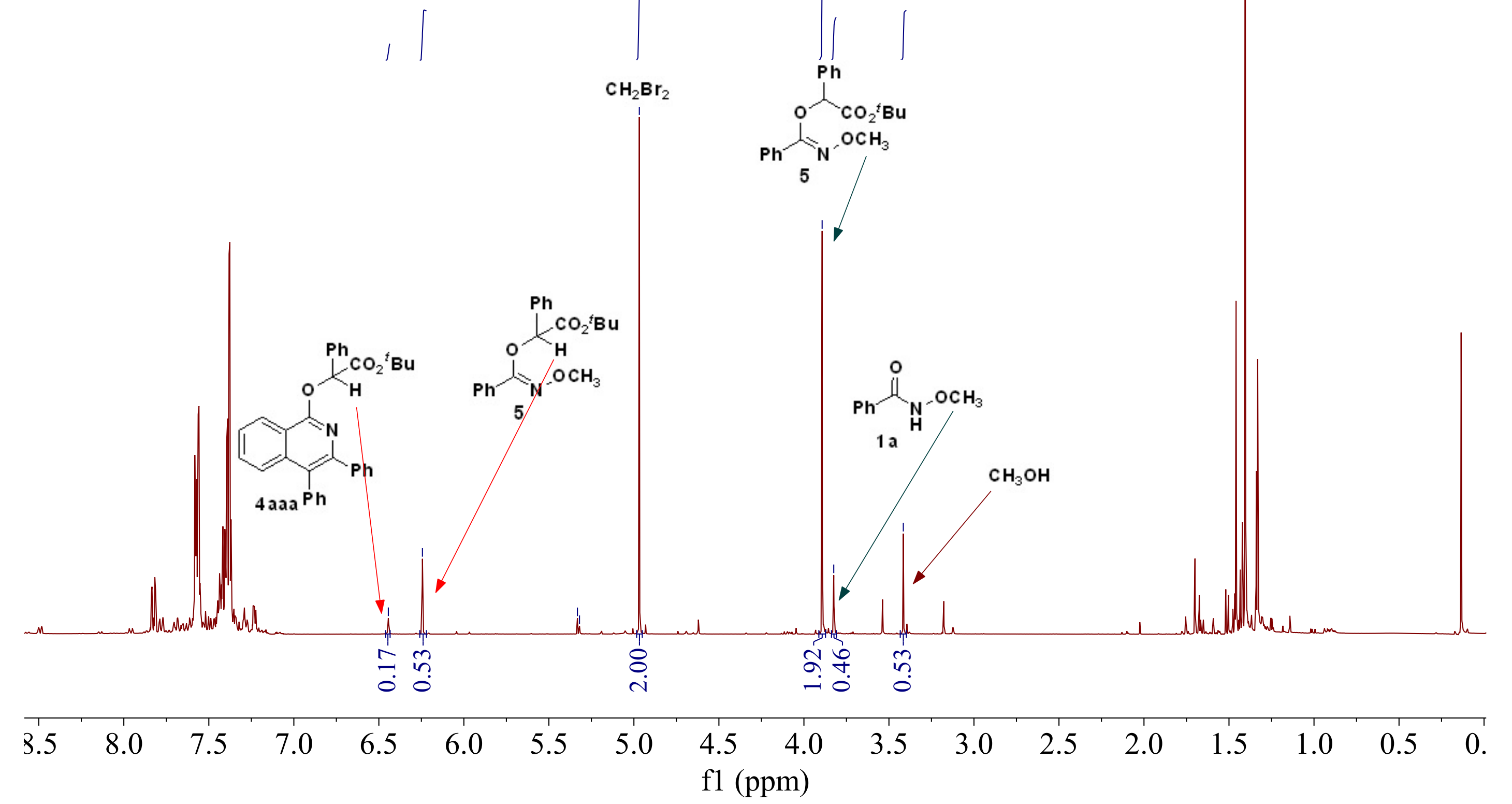


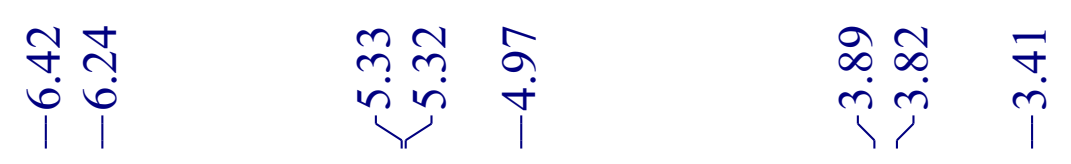

Reaction time: $3.5 \mathrm{~h}$

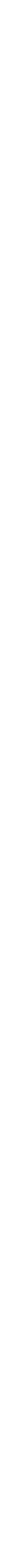


Reaction time: $5.0 \mathrm{~h}$

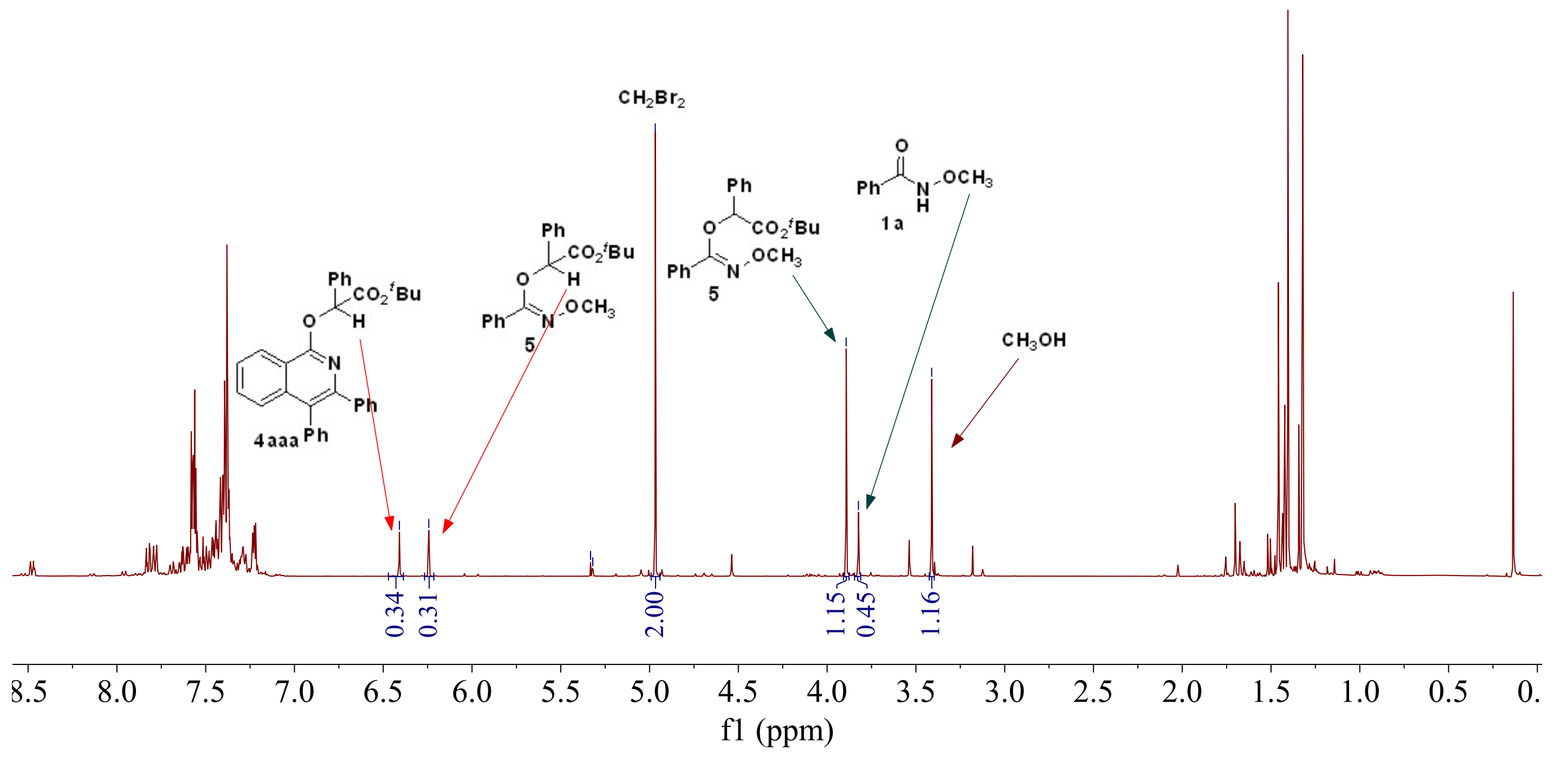




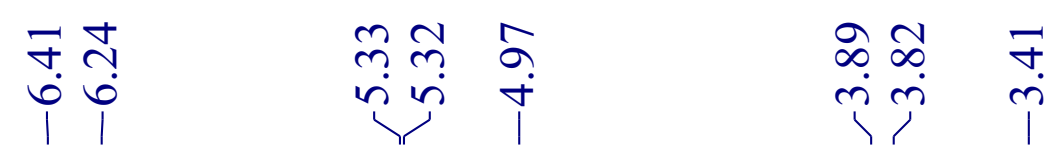

Reaction time: $5.5 \mathrm{~h}$

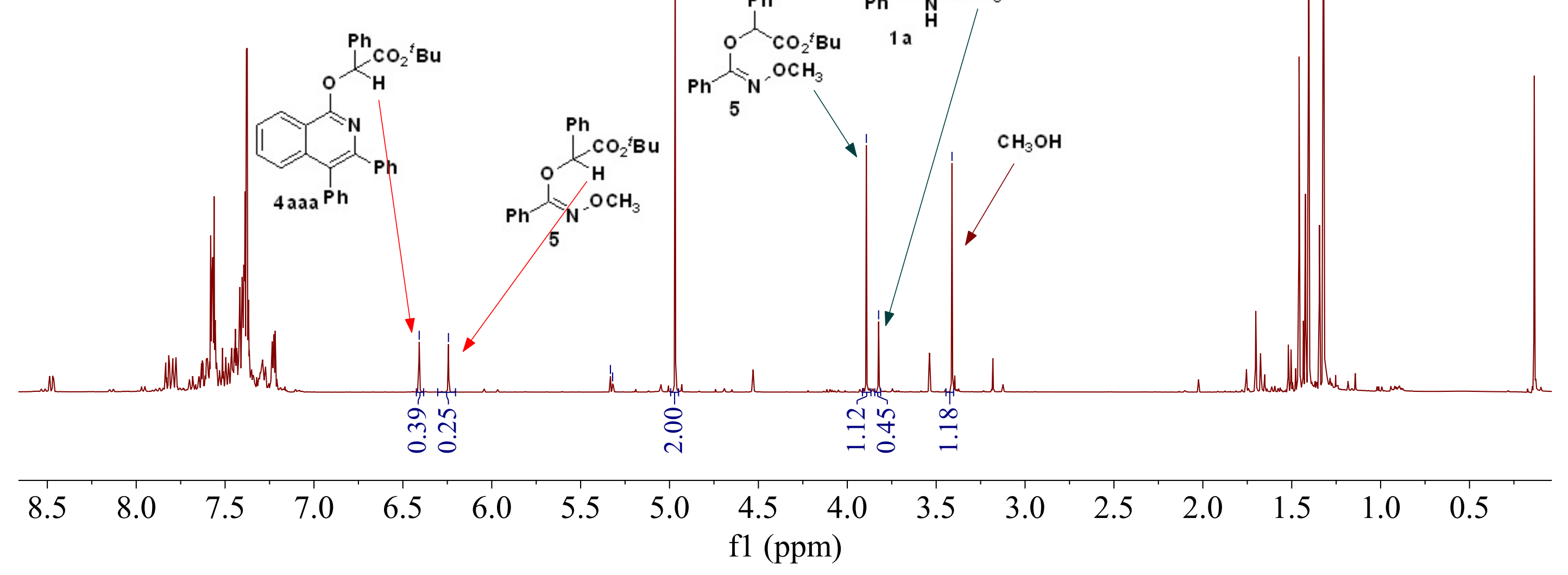




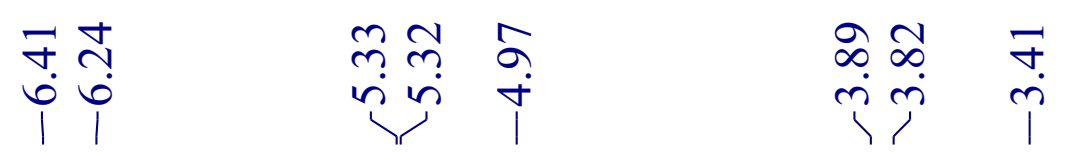

Reaction time: $7.5 \mathrm{~h}$

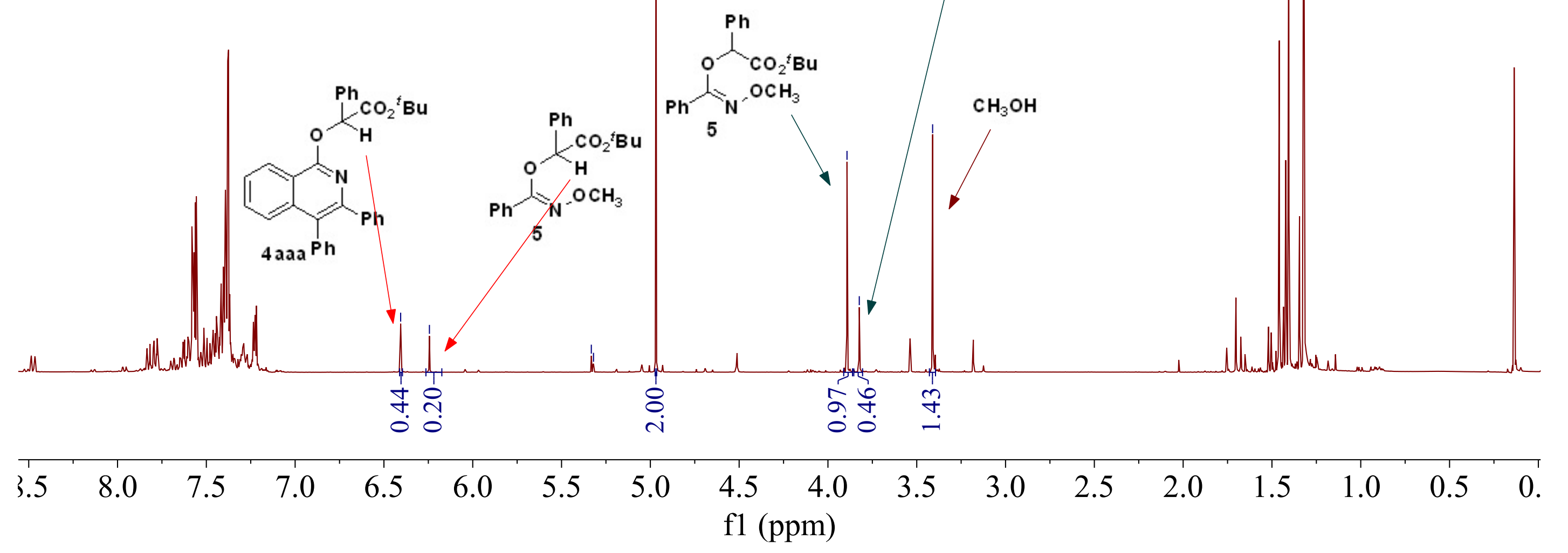




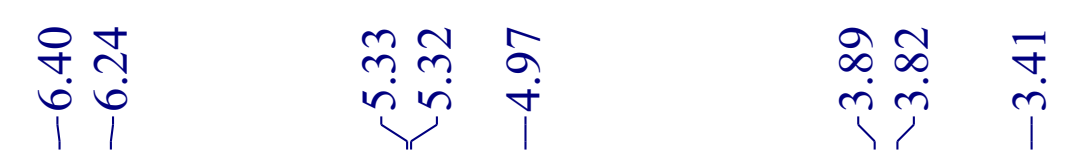

Reaction time: $19.0 \mathrm{~h}$
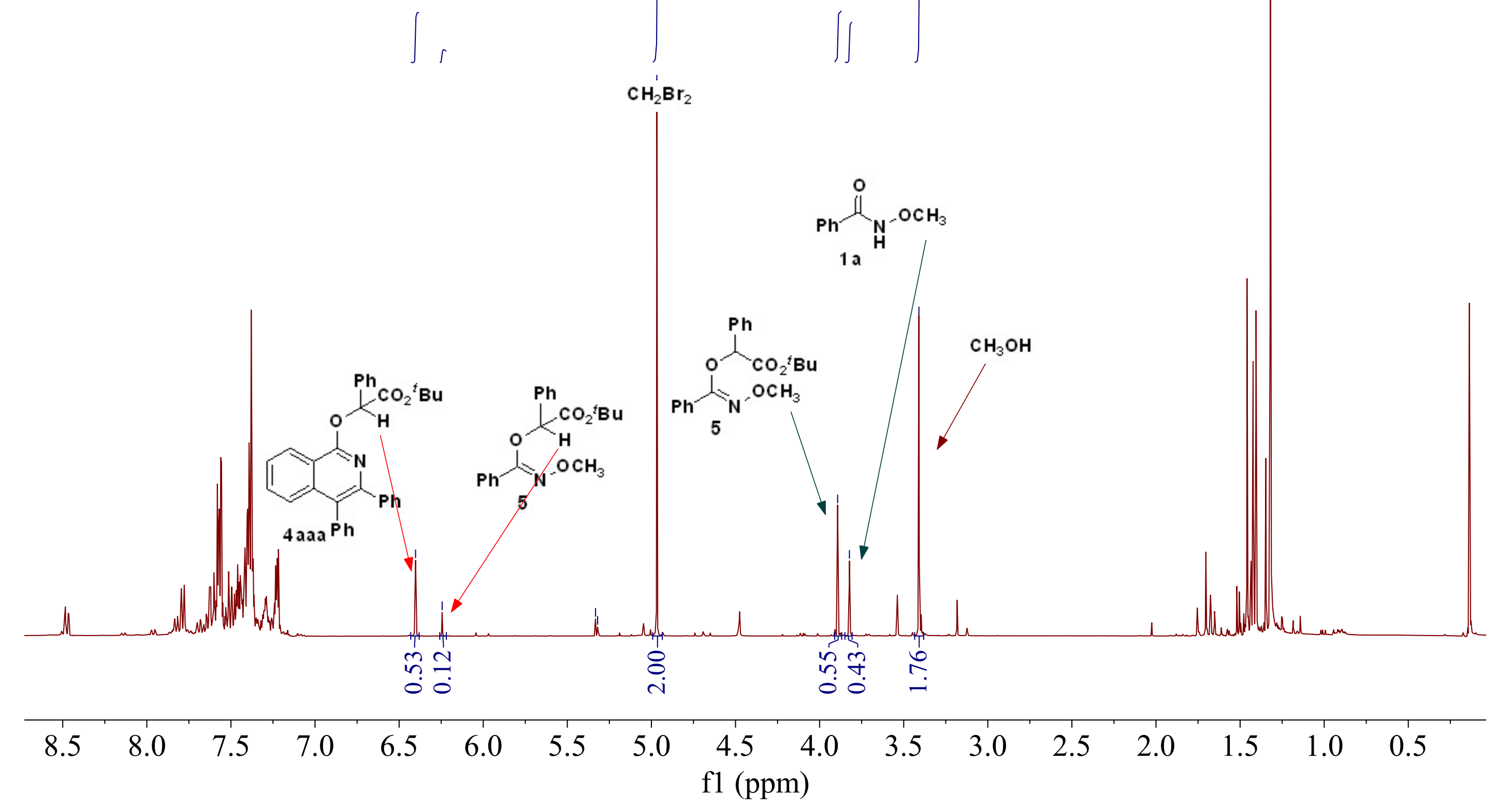